\title{
STRAIN MEASUREMENT OF COMPOSITE MATERIALS USING FIBRE OPTIC SENSORS
}

\author{
By \\ Peter Andrew Caskey Furlong
}

\author{
A thesis submitted to \\ The Faculty of Graduate Studies and Research \\ in partial fulfilment of \\ the degree requirements of \\ Master of Applied Science in Mechanical Engineering
}

Ottawa-Carleton Institute for

Mechanical and Aerospace Engineering

Department of Mechanical and Aerospace Engineering

Carleton University

Ottawa, Ontario, Canada

May 2007

(C) Copyright

2007 - Peter Andrew Caskey Furlong 


$\begin{array}{ll}\begin{array}{l}\text { Library and } \\ \text { Archives Canada }\end{array} & \begin{array}{l}\text { Bibliothèque et } \\ \text { Archives Canada }\end{array} \\ \begin{array}{l}\text { Published Heritage } \\ \text { Branch }\end{array} & \begin{array}{l}\text { Direction du } \\ \text { Patrimoine de l'édition }\end{array} \\ \begin{array}{l}\text { 395 Wellington Street } \\ \text { Ottawa ON K1A ON4 }\end{array} & \begin{array}{l}\text { 395, rue Wellington } \\ \text { Ottawa ON K1A ON4 } \\ \text { Canada }\end{array}\end{array}$

Your file Votre référence ISBN: 978-0-494-26987-9 Our file Notre référence ISBN: 978-0-494-26987-9

NOTICE:

The author has granted a nonexclusive license allowing Library and Archives Canada to reproduce, publish, archive, preserve, conserve, communicate to the public by telecommunication or on the Internet, loan, distribute and sell theses worldwide, for commercial or noncommercial purposes, in microform, paper, electronic and/or any other formats.

The author retains copyright ownership and moral rights in this thesis. Neither the thesis nor substantial extracts from it may be printed or otherwise reproduced without the author's permission.
AVIS:

L'auteur a accordé une licence non exclusive permettant à la Bibliothèque et Archives Canada de reproduire, publier, archiver, sauvegarder, conserver, transmettre au public par télécommunication ou par l'Internet, prêter, distribuer et vendre des thèses partout dans le monde, à des fins commerciales ou autres, sur support microforme, papier, électronique et/ou autres formats.

L'auteur conserve la propriété du droit d'auteur et des droits moraux qui protège cette thèse. $\mathrm{Ni}$ la thèse ni des extraits substantiels de celle-ci ne doivent être imprimés ou autrement reproduits sans son autorisation.
In compliance with the Canadian

Privacy Act some supporting forms may have been removed from this thesis.

While these forms may be included in the document page count, their removal does not represent any loss of content from the thesis.
Conformément à la loi canadienne sur la protection de la vie privée, quelques formulaires secondaires ont été enlevés de cette thèse.

Bien que ces formulaires aient inclus dans la pagination, il n'y aura aucun contenu manquant.




The undersigned recommend to

the Faculty of Graduate Studies and Research

acceptance of the thesis

\title{
Strain Measurement of Composite Materials Using Fibre Optic Sensors
}

\author{
Submitted by Peter Andrew Caskey Furlong \\ in partial fulfilment of the requirements for the degree of \\ Master of Applied Science in Mechanical Engineering
}

Dr. Choon-Lai Tan, Thesis Supervisor

Dr. Fred Nitzsche, Thesis Co-Supervisor

Dr. Jonathan Beddoes, Chair, Department of Mechanical and Aerospace Engineering

Carleton University

2007 


\section{ABSTRACT}

Fibre optic sensors are increasingly being used in aerospace and smart technology applications. Their compatibility with fibre reinforced composite materials and their high multiplexing abilities makes them particularly attractive as embedded strain sensors in aerospace structures. Integral fibre optic strain sensor arrays have been used to monitor strain cycling in horizontal-axis wind turbine rotor blade. Adaptation of this technology for use as integrated dynamic strain sensor networks within a fibre reinforced composite Mach scaled helicopter rotor blade presents, however, some unique challenges. In this thesis, an investigation into fibre optic strain sensory technology leads to the development of a conceptual fibre optic sensing system for this application, using the SHARCS rotor blade as example. As a major part of this thesis, a series of experiments is first conducted using fibre Bragg grating strain sensors on cantilever beams to verify their strain measurement ability. 


\section{I would like to dedicate this thesis to my father.}




\section{ACKNOWLEDGEMENTS}

I would like express my gratitude to my thesis supervisor, Dr. Tan, for his wisdom, guidance and patience throughout my entire graduate studies experience. I would also like to thank Dr. Nitzsche for introducing me to the SHARCS research project. I would like to thank Dr. Jacques Albert as well for graciously providing me with access to the necessary optical equipment which enabled me to carry out my experimental investigations. I wish also to thank Albane Laronch for fabricating and providing me with sensors, and for helping me to master the art of fusion splicing. Thanks are also due to Alex Proctor and Kevin Sangster for their guidance and assistance in the machine shop while fabricating my experimental test apparatus, specimens, and material test coupons. I thank Calvin Rans for assisting me with the tensile testing of the fibreglass material coupons. Finally I would like to thank Iain Johnston, Miriam Lyon, and the rest of the Carleton Triathlon Club organization for providing me with an essential outlet from my academic work; it's been a pleasure training and competing with you during my time here. 


\section{TABLE OF CONTENTS}

Abstract

Acknowledgements $\quad$ v

Table of Contents $\quad$ vi

List of Tables $\quad$ xi

List of Figures $\quad$ xii

Nomenclature $\quad$ xvi

Chapter 1: Introduction 1

Chapter 2: Fibre Optic Strain Sensors $\quad 5$

2.0 Introduction

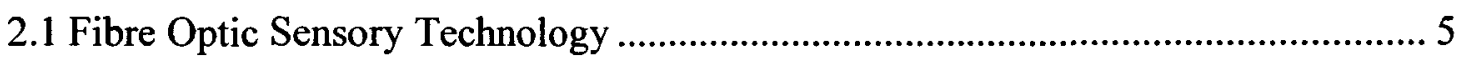

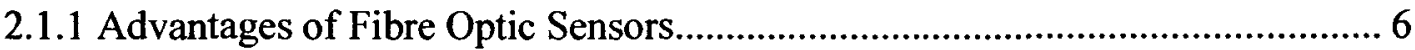

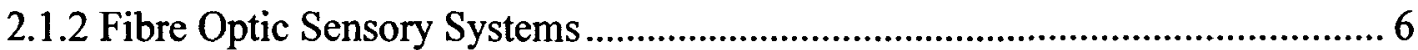

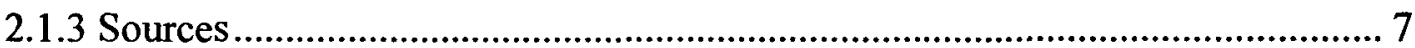

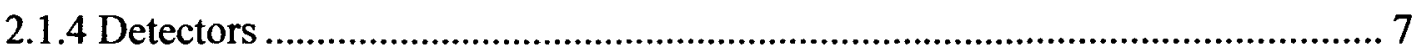

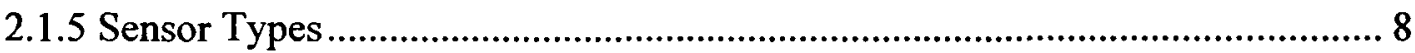

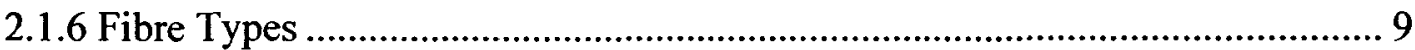

2.1.7 Optical Strain Sensors............................................................................... 9

2.1.8 Fibre Bragg Gratings (FBG) as Strain Sensors.......................................... 12

2.2 Review of Basic Fibre Optic Theory ............................................................ 13

vi 
2.2.1 The Nature of Light ..................................................................................... 14

2.2.2 Electromagnetic Waves .............................................................................. 15

2.2.3 Absorption of Light in Silica Glass ………………..................................... 15

2.2.4 Guiding Light within the Optical Fibre ........................................................ 17

2.2.5 Propagation of Guided Light ....................................................................... 18

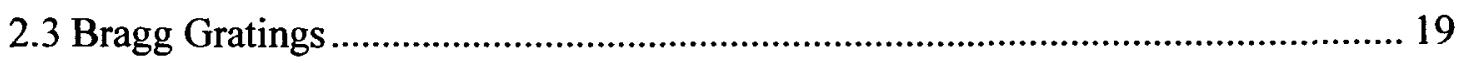

2.3.1 How Bragg Gratings are Written into Optical Fibre........................................ 20

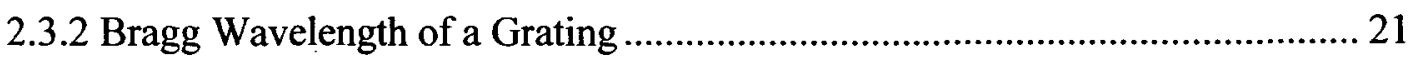

2.4 Measuring Mechanical Strain with Bragg Gratings.............................................. 22

2.4.1 Strain Response of a Bragg Grating.......................................................... 23

2.4.2 Thermal Response of a Bragg Grating............................................................ 24

2.4.3 Separation of Strain and Temperature Response ............................................ 25

2.4.4 Non-Uniform Strains and Gradients ............................................................. 26

2.4.5 Components of Strain ................................................................................. 27

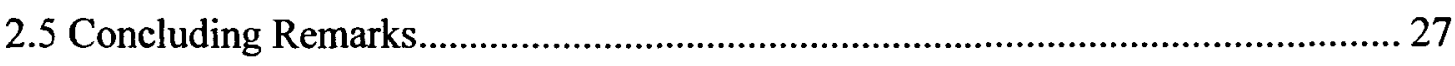

Chapter 3: Experimental Setup $\quad 36$

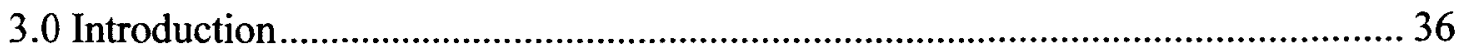

3.1 Equipment Setup and Integration....................................................................... 36

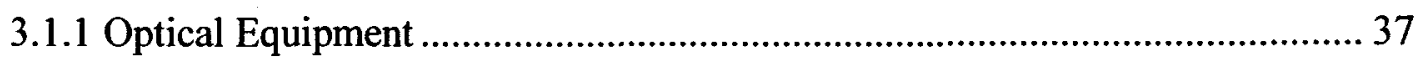

3.1.2 Electrical Resistance Strain Gauge Equipment............................................... 38

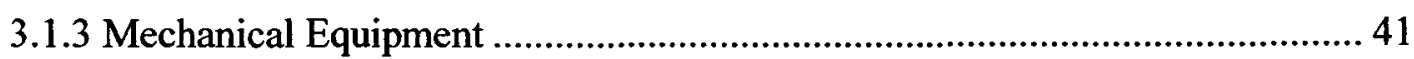

3.2 Specimen Geometry and Constraints.................................................................. 42

3.2.1 Aluminum Specimen Geometry and Constraints ........................................... 42

3.2.2 Fibreglass Specimen Geometry and Constraints ............................................. 43

3.3 Applied Loads .............................................................................................. 44



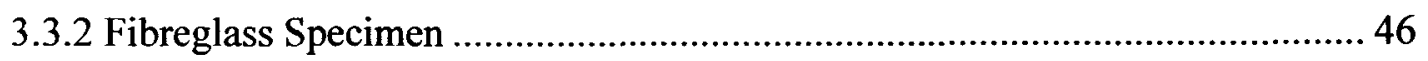

3.4 Material Properties of Specimens ....................................................................... 47

3.4.1 Aluminum Properties .............................................................................. 50

vii 


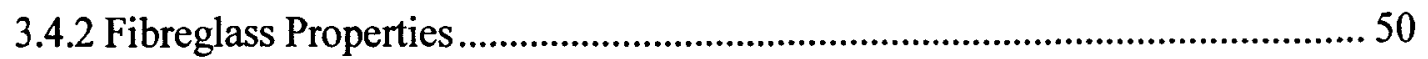

3.5 Cantilever Beam Theoretical Solutions ..................................................................5

3.5.1 Bending of a Hollow Rectangular Cantilever Beam.......................................... 53

3.5.2 Torsion of a Thin-Walled Hollow Rectangular Section .................................... 54

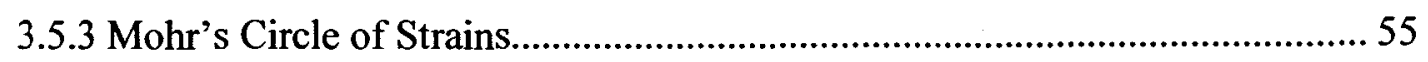

3.6 Strain Gauge Rosette Measurements .................................................................. 56

3.6.1 Installation of Electrical Resistance Gauges....................................................58

3.6.2 Aluminum Specimen .................................................................................... 59

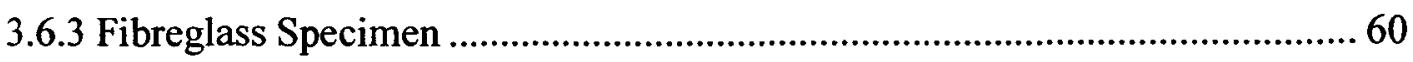

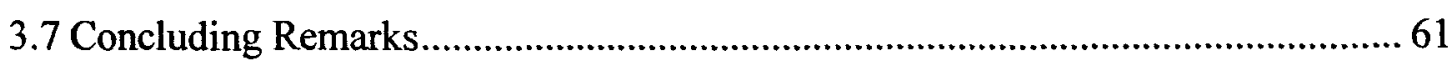

$\begin{array}{lr}\text { Chapter 4: FBG Sensor Testing and Results } & 88\end{array}$

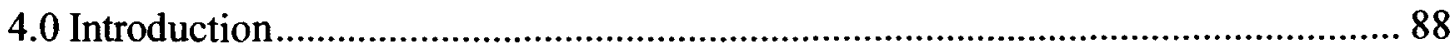

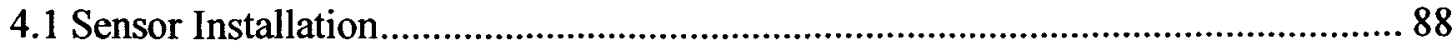

4.2 Sensor Properties and Optical Signals ................................................................ 92

4.3 Collection and Processing of Optical Signal Data ................................................. 93

4.4 Sensor Experimental Results ......................................................................... 95

4.4.1 Aluminum Specimen ................................................................................ 95

4.4.2 Fibreglass Specimen ................................................................................ 96

4.5 Discussion of Results and Errors ......................................................................... 99

4.5.1 Data Collection and Processing ....................................................................... 99

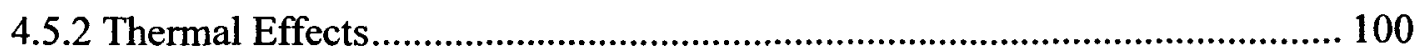

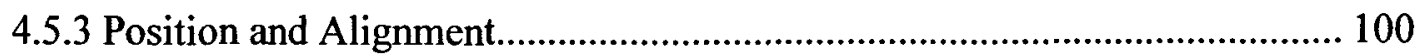

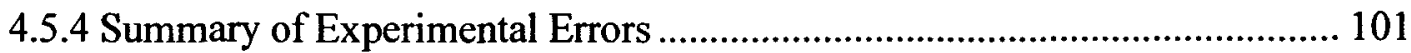

4.6 Transverse Sensitivity of FBG Sensors ............................................................ 102

4.6.1 Transverse Sensitivity Behaviour Observed in Experimental Results .......... 104

4.6.2 Transverse Sensitivity of Embedded Sensors in a Laminate........................... 107

4.7 Applying FBG Sensors to Thin Shell Laminates.................................................... 111

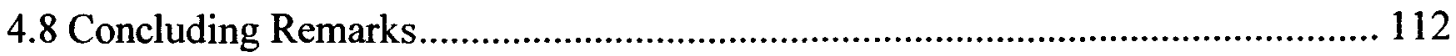

viii 


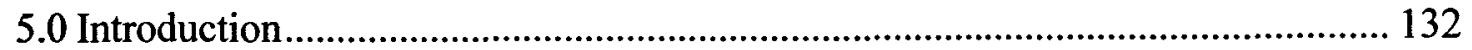

5.1 System Design Objectives and Basic Requirements ........................................... 133

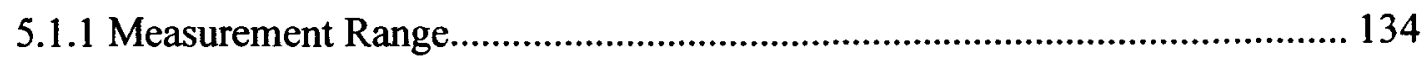

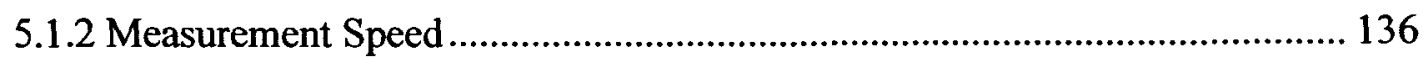

5.1.3 Measurement Resolution ........................................................................... 137

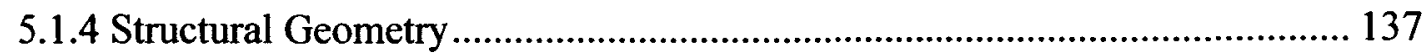

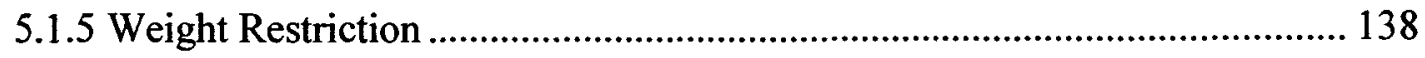

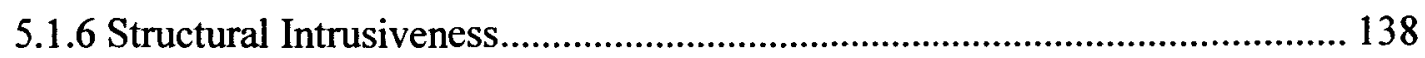

5.1.7 Summary of Design Requirements ............................................................. 138

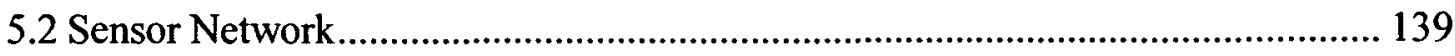

5.2.1 Dynamic Strain Monitoring Sensors............................................................. 139

5.2.2 Vibration Monitoring Sensors.................................................................... 140

5.2.3 Structural Health Monitoring Sensors ........................................................... 141

5.2.4 Combining all Sensors into a Single Network ............................................. 142

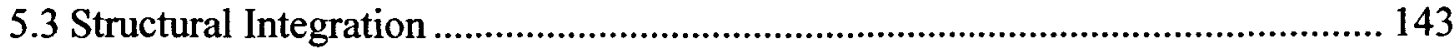

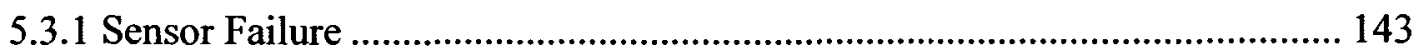

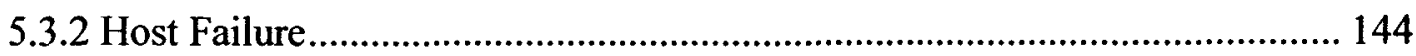

5.3.3 Routing of Optical Fibre in the SHARCS Rotor ......................................... 145

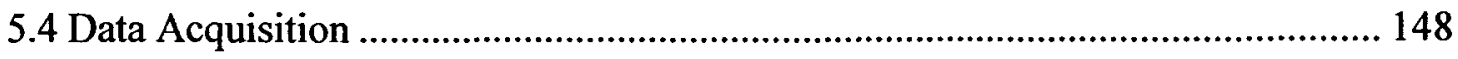

5.4.1 Transmitting and Receiving Optical Signals ................................................. 148

5.4.2 Optical Circuit Components ......................................................................... 149

5.4.3 SHARCS Sensory System Concept ........................................................... 152

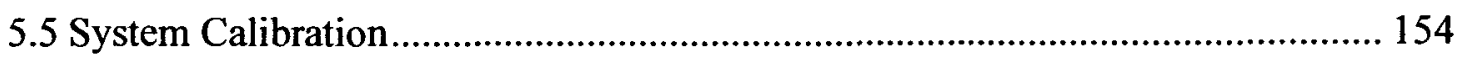

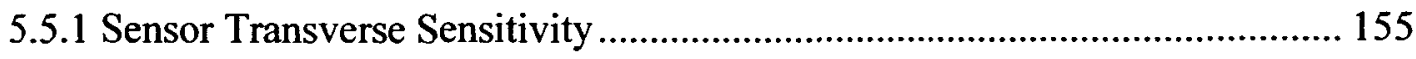

5.5.2 Wavelength Dependence of Fused Couplers/Splitters.................................... 155

5.5.3 Temperature Response of a LPG ............................................................... 156

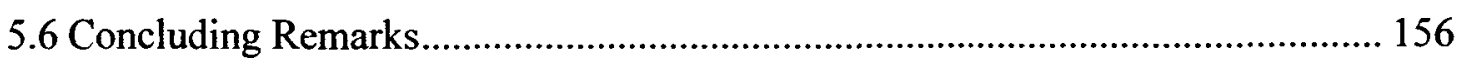

Chapter 6: Conclusions $\quad 163$

ix 


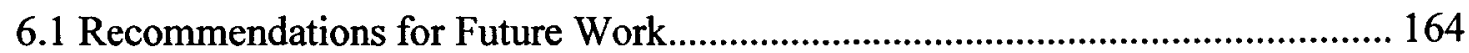




\section{LIST OF TABLES}

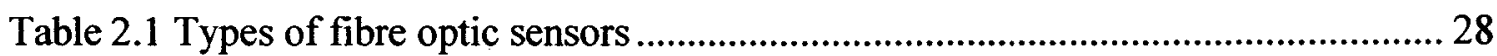

Table 2.2 Typical categories of the electromagnetic spectrum........................................... 28

Table 3.1 Aluminum specimen applied loads ................................................................. 62

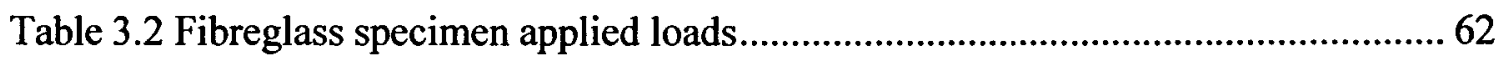

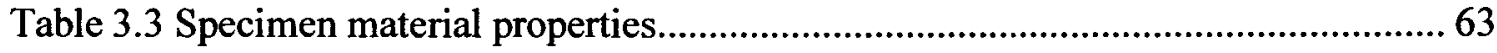

Table 3.4 Test specimen electrical resistance gauge rosette information............................ 63

Table 3.5 Principal strains on the aluminum specimen measured by the top rosette ....... 64

Table 3.6 Principal strains on the aluminum specimen measured by the bottom rosette. 64

Table 3.7 Principal strains on the fibreglass specimen in poor agreement......................... 65

Table 3.8 Principal strains on the fibreglass specimen in good agreement ......................... 65

Table 4.1 Typical properties of experimental materials used in FBG experiments......... 113

Table 4.2 Fibre Bragg grating sensor information........................................................... 113

Table 4.3 Sample of raw optical data...................................................................... 114

Table 4.4 FBG sensor measurements on the aluminum beam........................................ 114

Table 4.5 FBG sensor measurements on the fibreglass beam .......................................... 115

Table 5.1 Lamina material properties used for SHARCS rotor - Mikjaniec (2006)...... 157

Table 5.2 Lamina stiffness matrix coefficients........................................................... 157

Table 5.3 Approximate extension stiffness matrices of SHARCS rotor .......................... 158

Table 5.4 Sensitivity coefficients for embedded FBG sensor in lamina.......................... 158 


\section{LIST OF FIGURES}

Figure 1.1 SHARCS rotor blade and sub-systems - Mikjaniec (2006).............................. 4

Figure 1.2 Wind turbine with fibre optic sensors - Schroeder et al. (2006) ......................... 4

Figure 2.1 Typical optical circuit using a tuneable laser source....................................... 29

Figure 2.2 Typical optical circuit using a broadband source ............................................ 29

Figure 2.3 Propagation of an electromagnetic wave......................................................... 30

Figure 2.4 Interaction of light with a substance.......................................................... 30

Figure 2.5 Signal attenuation of silica optical fibre - Hecht (2006)................................. 31

Figure 2.6 Reflection, refraction and critical angle for total internal reflection ................ 31

Figure 2.7 Typical cross-section of a step-indexed multi-mode silica optical fibre......... 32

Figure 2.8 Different acceptable propagation paths of guided rays in an optical fibre...... 32

Figure 2.9 Modal representation of guided light in an optical fibre ............................... 33

Figure 2.10 Phase mask fabrication technique - Hill et al. (1997)................................... 33

Figure 2.11 Fibre Bragg grating................................................................................. 34

Figure 2.12 Wavelength division multiplexed array of Bragg grating sensors ................ 34

Figure 2.13 Variation of thermo-optic coefficient for silica fibre - Hill et al. (1997)...... 35

Figure 3.1 Optical interrogation circuit ....................................................................... 66

Figure 3.2 Optical signal sample of a fibre Bragg grating................................................6

Figure 3.3 Wire grid type electrical resistance strain gauge ..............................................67

Figure 3.4 Wheatstone bridge circuit.......................................................................... 67

Figure 3.5 Single gauge in quarter bridge configuration ..................................................68

Figure 3.6 Portable strain indicator and switch and balance units.................................... 68

Figure 3.7 Steel support frame.................................................................................... 69

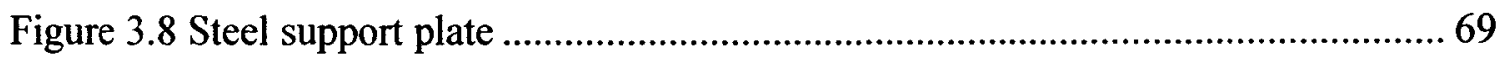

Figure 3.9 Aluminum section: geometric properties ……………………....................... 70 
Figure 3.10 Fibreglass section: geometric properties ................................................... 70

Figure 3.11 Fibreglass beam: fixtures and constraints...................................................... 71

Figure 3.12 Resolving applied loads................................................................................. 71

Figure 3.13 Material test coupon geometry of the fibreglass used ................................... 72

Figure 3.14 Axial stress versus axial strain plot for fibreglass test coupon....................... 72

Figure 3.15 Strain coordinate transformation relationship and Mohr's circle ................... 73

Figure 3.16 Principal strain orientation and Mohr's circle .............................................. 73

Figure 3.17 Typical electrical resistance strain gauge rosette configurations ................... 74

Figure 3.18 Construction of Mohr's circle using a rectangular strain gauge rosette ........ 74

Figure 3.19 Bending strains measured by the top rosette for upright aluminum beam .... 75

Figure 3.20 Combined bending and torsion strains measured by the top rosette for upright

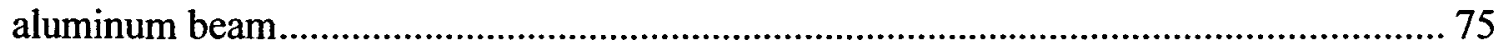

Figure 3.21 Principal angles measured by the top rosette for upright aluminum beam ... 76 Figure 3.22 Bending strains measured by the top rosette for inverted aluminum beam .. 76 Figure 3.23 Combined bending and torsion strains measured by the top rosette for

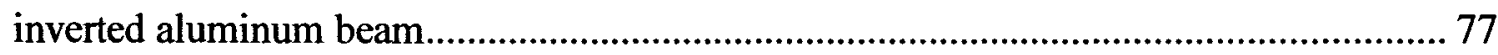

Figure 3.24 Principal angles measured by the top rosette for inverted aluminum beam.. 77 Figure 3.25 Bending strains measured by the bottom rosette for upright aluminum beam

Figure 3.26 Combined bending and torsion strains measured by the bottom rosette for

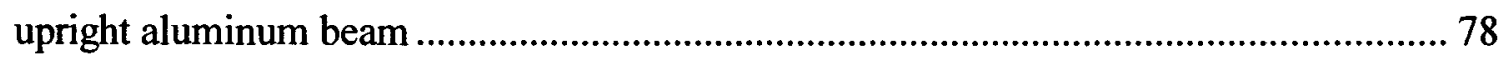

Figure 3.27 Principal angles measured by the bottom rosette for upright aluminum beam

Figure 3.28 Bending strains measured by the bottom rosette for inverted aluminum beam

Figure 3.29 Combined bending and torsion strains measured by the bottom rosette for inverted aluminum beam. 80

Figure 3.30 Principal angles measured by the bottom rosette for inverted aluminum beam 
Figure 3.31 Bending strains in poor agreement for upright fibreglass beam.

Figure 3.32 Combined bending and torsion strains in poor agreement for upright

fibreglass beam

Figure $3.33 \alpha=0$ degree direction axial strains in poor agreement for fibreglass beam.. 82

Figure $3.34 \alpha=45$ degree direction axial strains in poor agreement for fibreglass beam 82

Figure $3.35 \alpha=90$ degree direction axial strains in poor agreement for fibreglass beam 83

Figure $3.36 \alpha=0$ degree direction axial strains in good agreement for fibreglass beam. 83

Figure $3.37 \alpha=45$ degree direction axial strains in good agreement for fibreglass beam 84

Figure $3.38 \alpha=90$ degree direction axial strains in good agreement for fibreglass beam 84

Figure 3.39 Bending strains in good agreement for upright fibreglass beam. 85

Figure 3.40 Combined bending and torsion strains in good agreement for upright

fibreglass beam 85

Figure 3.41 Principal angles in good agreement for upright fibreglass beam 86

Figure 3.42 Bending strains in good agreement for inverted fibreglass beam .86

Figure 3.43 Combined bending and torsion strains in good agreement for inverted fibreglass beam 87

Figure 3.44 Principal angles in good agreement for inverted fibreglass beam. 87

Figure 4.1 Installation of a FBG using different adhesives 116

Figure 4.2 Optical signal change of tilted FBG \#1 during installation. 116

Figure 4.3 Optical signal of FBG \#1 after installation 117

Figure 4.4 Optical signal of FBG \#2 after installation. 117

Figure 4.5 Optical signal of FBG \#3 after installation. 118

Figure 4.6 Optical signal of FBG \#4 after installation. 118

Figure 4.7 Optical signal of FBG \#5 after installation 119

Figure 4.8 Optical signal of FBG \#6 after installation 119

Figure 4.9 Optical signal of FBG \#7 after installation. 120

Figure 4.10 Optical signal of FBG \#8 after installation 120

Figure 4.11 FBG reflection signals aligned using maximum insertion loss 121

Figure 4.12 FBG reflection signals aligned using centre wavelength 121 xiv 
Figure 4.13 Shift of FBG \#4 optical signal due to applied strain .............................. 122

Figure 4.14 Error in FBG strain readings due to thermal sensitivity........................... 122

Figure 4.15 Bending results for axial strain on FBG \#1 ........................................ 123

Figure 4.16 Combined bending and torsion results for axial strain on FBG \#1 ........... 123

Figure 4.17 Bending results for axial strain on FBG \#2 …................................... 124

Figure 4.18 Combined bending and torsion results for axial strain on FBG $\# 2$............ 124

Figure 4.19 Bending results for axial strain on FBG \#3 ........................................... 125

Figure 4.20 Combined bending and torsion results for axial strain on FBG \#3 ........... 125

Figure 4.21 Bending results for axial strain on FBG \#4 .......................................... 126

Figure 4.22 Combined bending and torsion results for axial strain on FBG \#4 ........... 126

Figure 4.23 Bending results for axial strain on FBG \#5 …....................................... 127

Figure 4.24 Combined bending and torsion results for axial strain on FBG \#5 ........... 127

Figure 4.25 Bending results for axial strain on FBG \#6 ........................................ 128

Figure 4.26 Combined bending and torsion results for axial strain on FBG \#6 ........... 128

Figure 4.27 Bending results for axial strain on FBG \#7 f.......................................... 129

Figure 4.28 Combined bending and torsion results for axial strain on FBG \#7 ............ 129

Figure 4.29 Bending results for axial strain on FBG \#8 ........................................... 130

Figure 4.30 Combined bending and torsion results for axial strain on FBG \#8 ............ 130

Figure 4.31 Transverse stresses acting on the FBG sensors due to adhesive ................. 131

Figure 4.32 FBG sensors embedded in a unidirectional laminate, Fan et al. (2004)...... 131

Figure 5.1 Laminate lay-up for the root of SHARCS rotor - Mikjaniec (2006) ............ 159

Figure 5.2 Laminate lay-up for the blade of SHARCS rotor - Mikjaniec (2006) .......... 159

Figure 5.3 Sensor placement and optical fibre routing for SHARCS rotor ................. 160

Figure 5.4 Long period grating transmission signal - Bhatia (1999) ............................. 161

Figure 5.5 Basic LPG interrogation circuit for a FBG sensor ................................... 161

Figure 5.6 Conceptual data acquisition system for SHARCS ................................. 162 


\section{NOMENCLATURE}

\begin{tabular}{|c|c|}
\hline$b_{i}, b_{o}$ & width of the interior and outer walls of the cross-section \\
\hline$c$ & speed of light in a vacuum \\
\hline$h_{i}, h_{o}$ & height of the interior and outer walls of the cross-section \\
\hline$k_{\text {opt }}$ & transverse sensitivity coefficient \\
\hline$k_{E l}$ & coefficient relating the elastic influence of a lamina on a sensor \\
\hline$l_{k}$ & length of the $\mathrm{k}^{\text {th }}$ lamina \\
\hline$n_{e}$ & effective refractive index of the fibre core \\
\hline$n_{i}$ & refractive index of the $i$ th medium \\
\hline$r_{\text {bend }}$ & bending radius of an optical fibre \\
\hline$r_{L E}$ & radius of the leading edge of a rotor blade cross-section \\
\hline$t_{k}$ & thickness of the $\mathrm{k}^{\text {th }}$ lamina \\
\hline$t_{y}$ & thickness of the top surface wall of the beam \\
\hline$v_{i}$ & speed of light in the $i$ th medium \\
\hline$x$ & position in the $x$ axis direction \\
\hline$y_{o}, y_{i}$ & position on the outer and interior surfaces in the $y$ axis direction \\
\hline$z$ & position in the $z$ axis direction \\
\hline
\end{tabular}

xvi 
area of the cross-section in the $x-y$ plane

$A_{11}, A_{12}, A_{22} \quad$ coefficients of the laminate extension stiffness matrix

$C W \quad$ centre wavelength of an optical signal

$E_{i} \quad$ Young's modulus for the $i$ th direction

F axial applied axial force on cross-section of rotor

$F_{a}, F_{t} \quad$ axial and transverse strain sensitivity factors

$F_{o p t} \quad$ FBG gauge factor coefficient

$G_{i j} \quad$ shear modulus 'ij'

$I_{x} \quad$ second moment of area of cross-section about the $x$ axis

$K_{13 l}, K_{23 l} \quad$ coefficients relating the direct strains of a lamina in plane stress

$L \quad$ length

$M_{x}, T_{z} \quad$ moment about the $x$ axis and torque about the $z$ axis

$P_{i} \quad$ force applied in the $i$ th direction

$P_{i j} \quad$ strain-optic coefficients of the fibre core

$P_{e} \quad$ effective strain-optic coefficient of the fibre core

$R \quad$ radius

$\Delta R \quad$ change in electrical resistance

$\Delta T \quad$ change in temperature

$\left[T_{f a}\right] \quad$ matrix regarding transfer of strain from the adhesive to the FBG

$\Delta V \quad$ change in voltage across Wheatstone bridge

xvii 


$\begin{array}{ll}\alpha_{s} & \text { coefficient of thermal expansion of the sensor fibre core } \\ \varepsilon_{r i} & \text { relative permittivity of the } i \text { th medium } \\ \varepsilon_{i j} & \text { strain component 'ij' } \\ \varepsilon_{i j}^{\prime} & \text { apparent strain component 'ij' } \\ \lambda_{B} & \text { Bragg resonance wavelength } \\ \Delta \lambda_{B} & \text { change in Bragg resonance wavelength } \\ \lambda_{R}, \lambda_{L} & \text { wavelengths at threshold value of an optical signal } \\ \mu_{r i} & \text { relative permeability of the } i \text { th medium } \\ v_{f}, v_{s} & \text { Poisson's ratio of the optical fibre } \\ \theta_{i} & \text { angular orientation ' } \mathrm{i} \text { ' } \\ \theta_{p a t h} & \text { angle of the lamina or optical fibre path to the rotor blade axis } \\ \sigma_{i j}, \tau_{i j} & \end{array}$

ACRONYMS

CLLIPS Carleton Laboratory for Laser Induced Photonic Structures

FBG Fibre Bragg Grating

FORJ Fibre Optic Rotary Joint

LED Light Emitting Diode

xviii 
LPG Long Period Grating

OTF Optical Tuneable Filter

PD Photo-Diode

SHARCS Smart Hybrid Active Rotor Control System

SLD Super Luminescent Diode

WDC Wavelength Dependant Coupler

xix 


\section{CHAPTER 1: INTRODUCTION}

Fibre optic sensors are finding increasingly more applications in aerospace and smart technology applications, from structural health monitoring to pressure, strain and temperature measurements. Their ability to be highly multiplexed into sensor arrays and their compatibility with fibre reinforced composite materials makes them highly attractive to experimental researchers as embedded sensors in aerospace structures.

An active area of research in the Department of Mechanical and Aerospace Engineering at Carleton University is the application of smart technology systems to controlling helicopter rotor blade structural dynamics. The Smart Hybrid Active Rotor Control System (SHARCS) was developed in order to simultaneously reduce vibration and noise by actively controlling three integrated smart sub-systems in a single rotor blade. The three smart sub-systems which make up SHARCS are the smart-spring, the anhedral tip and the trailing edge flap, as shown in figure 1.1.

One step in the development of SHARCS is experimental wind tunnel testing of its sub-systems using a one meter long fibre reinforced composite Mach scaled rotor blade, the preliminary design of which was carried out by Mikjaniec (2006); it is hereby referred to as the SHARCS rotor blade. The performance of SHARCS can be evaluated by dynamically monitoring the structural deformation and vibration behaviour of the rotor 
blade during wind tunnel testing. To this end, it is proposed that a distributed network of fibre optic strain sensors, embedded within its fibre reinforced composite structure be employed, thereby allowing simultaneous monitoring of the real-time strains at multiple locations. Systems have been developed for real-time load monitoring of horizontal-axis wind-turbine rotor blades, such as the system presented by Schroeder et al. (2006) for the wind-turbine shown in figure 1.2. This system collects the strain amplitude history of the rotor blade, which is useful in assessment of its fatigue life and continuing evaluation of its structural integrity. To adapt a similar fibre optic sensing system for use in a Mach scaled helicopter rotor blade, with the additional sensing ability to measure dynamic vibration amplitudes, presents a number of challenges. The rotor blade geometry is smaller, requiring a more compact system; its rotational speed is higher, requiring a faster data acquisition speed; its structure is more fragile, requiring a less intrusive sensor network; and the strain amplitudes associated with structural vibrations are significantly smaller in magnitude, requiring a more sensitive strain sensor.

The objective of this thesis is to investigate the feasibility of using fiber optic sensory technology specifically for use in the SHARCS rotor blade sensing application. The organization of this thesis will be as follows. The next Chapter will present a literature review of fibre optic strain sensor technology and devices, in which the most appropriate sensors for SHARCS are selected; the theoretical relationship which enables them to measure strain is also presented. Chapter 3 will present an experimental apparatus which will be used for testing the fibre optic strain sensors, which consists of two cantilever test specimens that are calibrated by use of conventional electrical resistance gauges. Chapter 
4 presents the experimental test results of the fibre optic strain sensors, and examines the agreement of the experimental measurements with the theoretical optical behaviour of the sensors. Chapter 5 presents an overview of the fibre optic strain sensing system design requirements based on the SHARCS rotor blade preliminary design. The associated challenges are discussed and a conceptual system capable of meeting the requirements is presented. The final conclusions from this work and recommendations for future work are then presented in Chapter 6. 


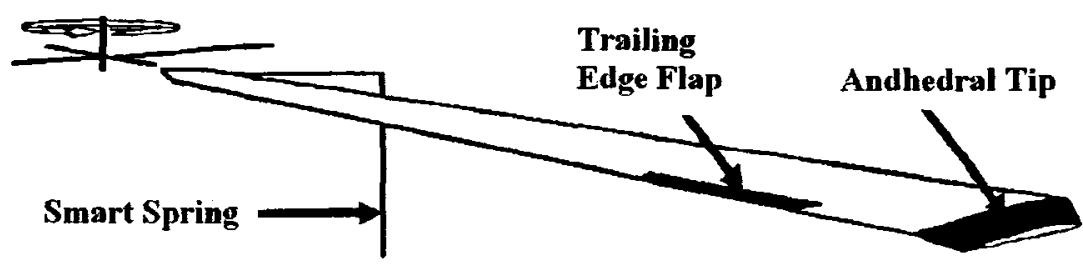

Figure 1.1 SHARCS rotor blade and sub-systems - Mikjaniec (2006)

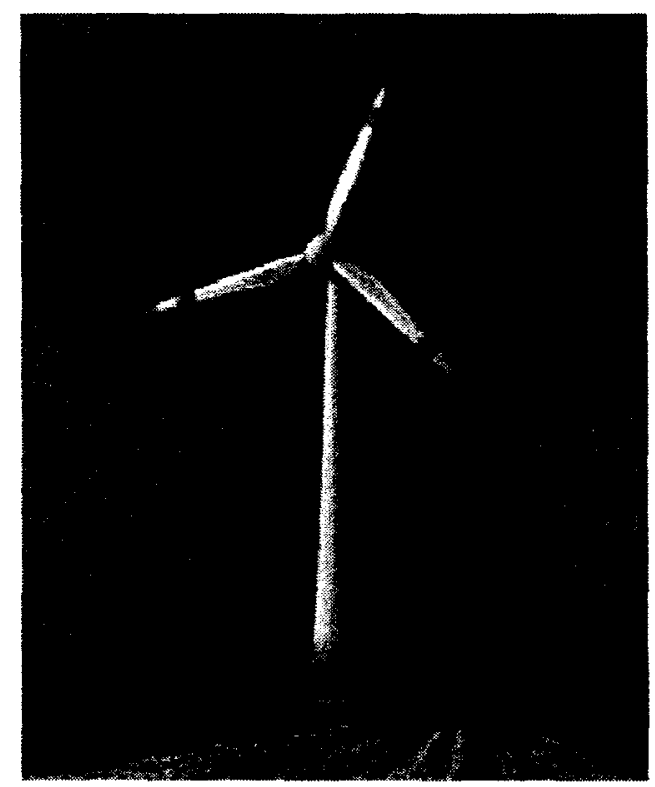

Figure 1.2 Wind turbine with fibre optic sensors - Schroeder et al. (2006) 


\section{CHAPTER 2: FIBRE OPTIC STRAIN SENSORS}

\subsection{Introduction}

A "fibre optic strain sensor" is a device which employs the use of fibre optics to measure mechanical strain. It is a part of a much larger family of "fibre optic sensors" of which there are many device types and measurands. This Chapter provides a brief background on fibre optic sensory technology, with particular focus on strain measurement devices. The focus is further concentrated on some of the research work that has been done on the development of fibre Bragg gratings as strain sensors. To this end, a basic review of fibre optic theory is also provided in this Chapter to serve as background for understanding how fibre Bragg gratings work.

\subsection{Fibre Optic Sensory Technology}

Fibre optic sensory technology is still in its adolescence; it has emerged out of the fibre optic revolution that occurred in the telecommunications industry through the 1970 s and 1980s. The tremendous consumer demand in telecommunications has since continued to drive the development of fibre optic technology at an incredible rate. Udd (1991) has shown that this high-paced development has led to a drastic reduction in the cost associated with fibre optic equipment, a significant increase in component quality, 
variety, and availability. All of these factors combined show an exceptional market trend for fibre optics to strongly set foot in the sensory industry.

\subsubsection{Advantages of Fibre Optic Sensors}

Udd (1991) describes many of the advantages associated with fibre optic sensors over conventional methods. They include their immunity to electromagnetic interference, high-temperature performance, vibration and shock resistance, chemical resistance, compact size, light weight, high sensitivity, high accuracy, high speed potential, low signal loss over large distances, and ability to be highly multiplexed into large networks. For these and other reasons, it is believed that fibre optic sensors show great potential for replacing the majority of environmental sensory equipment in use; in some cases, they even show potential for creation of new sensors that could produce such quality of measurement that other sensory devices simply could not compare.

\subsubsection{Fibre Optic Sensory Systems}

A typical fibre optic sensory system consists of four major elements; the source, the optical fibre, the sensor, and the detector. The combination of these elements can vary significantly depending on the desired accuracy of measurement, and the required speed of data acquisition. The most noticeable difference between systems is the type of source used, and typically falls into one of two categories; a tuneable laser source, or a broadband light source. Gornall (2003) shows the basic optical circuit diagrams for these two different system styles, as outlined in figures 2.1 and 2.2 respectively. 


\subsubsection{Sources}

A tuneable laser source provides a single wavelength of light as the optical input, and can be 'tuned', or swept through a range of wavelengths. A broadband light source simply provides many wavelengths of light simultaneously and continuously as the optical input. It relies entirely on the optical spectrum analyzer for determining both power and wavelength of the optical sensor output signals. Tuneable laser sources are typically more expensive and bulky, but provide higher measurement accuracy, and are thus more suited to laboratory testing and characterization of the optical sensor devices through controlled experiments. Broadband light sources are more compact and rugged, and thus offer a more portable system for doing field measurements during practical application of the sensor technology.

\subsubsection{Detectors}

Tuneable laser sources are typically combined with two detector components; a power meter and a wavelength measurement feedback device. The feedback device, which might be packaged directly with the laser source itself, provides improved wavelength accuracy while sweeping. The power of the output signal is measured by a power meter, independent of wavelength, making for a fairly simple detection system.

Broadband light sources are typically combined with optical spectrum analyzers, which according to Agilent Technologies (1996), typically fall into one of three categories, namely diffraction-grating-based, Fabry-Perot interferometer-based or Michelson interferometer-based. Of these three, Gornall (2003) claims the Michelson 
interferometer-based analyzers usually offer the highest accuracy of wavelength measurement.

Alternative spectrum analysis techniques are continuously being sought and developed for specific sensing applications. These alternatives would provide cheaper, more compact, higher speed, more accurate, or simpler methods for detection, thereby better meeting the exact needs for data acquisition of the system. Examples of such systems are (a) the one suggested by Kang et al. (1998), which uses a tilted fibre Bragg grating mounted on a piezo-ceramic stretching element to act as a tuneable filter for interrogating another sensor, and (b) the one suggested by Simpson et al. (2004), which uses a low-cost charge-coupled device linear array to measure the radiation modes exiting a tilted fibre Bragg grating, allowing for high accuracy interrogation of multiple sensors. When combined with the already compact nature of broadband light sources and the sensor devices themselves, these alternatives could lead to affordable optical sensing systems with the ability to be fully integrated into the sensing environment or structure.

\subsubsection{Sensor Types}

While the selection of source and detector components is extremely important to achieving the desired speed and accuracy of the system, it is the sensors themselves that must be most carefully selected to match the desired measurands. Table 2.1 lists a variety of sensor types and some of the measurands that Udd (1991) associates with each.

Optical sensors can be classified as either extrinsic or intrinsic. For the extrinsic type, the sensing takes place outside of the fibre, which is used solely as a conduit to transport light signals to and from the sensor device. For the intrinsic sensors, also known as all- 
fibre sensors, the sensing takes place within the fibre itself. Intrinsic sensors have created a large wake of research because of their simple all-fibre design, allowing them to be easily combined with fibre reinforced composite structures.

\subsubsection{Fibre Types}

Intrinsic sensors can be created in a large variety of optical fibre materials and types. The most commonly used optical material is silica glass fibre because of its abundant use in telecommunications applications. It typically has additives, known as dopants, such as germanium to enhance the optical properties of the base silica fibre. Silica fibre is commercially available in many varieties, such as single-mode, multi-mode, step or graded index profiles, high birefringence or polarization preserving. The most commonly used varieties are the step-index multi-mode and step-index single-mode. There are other more exotic optical materials that have been researched for sensor use, for example, Xiao et al. (2003), and Grobnic et al. (2004) suggest the use of single-crystal sapphire devices for taking strain measurements under high temperature conditions where the doped silicabased sensors cannot maintain consistent optical properties.

\subsubsection{Optical Strain Sensors}

Civil engineers have applied fibre optic strain sensors to large concrete structures like buildings, bridges and some roadways for monitoring deflections, strains, and thermal expansion for some time, Gornall et al. (2003). Many of these devices offered several advantages; they include the ability of the optical fibres to be highly multiplexed, and be distributed easily over large distances with low signal loss. An example of the type of 
instrument designed for civil engineering applications is an extensometer that uses an internal fibre Bragg grating as a strain measurement device.

The large scale of civil structures has allowed embedment of fibre optic cable and sensors with minimal intrusiveness and negligible effects on the overall integrity and behaviour of the structure. However, the same may not be true for use in smaller and thinner composite structures that are typical of the aerospace and many mechanical engineering applications. The embedment of the optical fibre and sensing devices can have a significant effect on the behaviour of the final structure. For intrinsic devices, it would only be necessary to determine the effects of embedding the optical fibre into a composite structure, thereby reducing the complexity of the problem to modeling a simple continuous fibre as an inclusion.

Some work has been done by mechanical and aerospace engineers to address the mechanical performance of composite structures with these embedded all-fibre sensors. Eaton et al. (1995) examined by using finite element analysis the induced stress and strain concentrations in both the optical fibre, and the surrounding composite laminate structure due to embedment at several relative fibre orientations. Surgeon et al. (2001) performed some experimental work that examined the influence of an embedded optical fibre on fatigue damage progress in carbon fibre reinforced polymers. Skontorp (2002) investigated the embedment of optical fibre at structural details with inherent stress concentrations, and concluded that the presence of the optical fibre, when oriented in the primary direction of the laminate's fibre, did not significantly affect the structural integrity of the laminate, or initiate its failure. Shivakumar et al. (2004) investigated 
experimentally the failure modes and effects of composite laminate coupons with an embedded optical fibre at different orientations. Later, Shivakumar et al. (2005) used finite element models of the same test coupons to predict the stress concentrations, and the failure of those specimens due to the inclusion of the optical fibre. This work continues in parallel to the development and refinement of the intrinsic strain sensors themselves.

Of the intrinsic fibre optic strain sensors listed in Table 2.1, there are three of particular interest; the Brillouin scattering, the interferometric type, and the fibre Bragg grating type sensors. The Brillouin scattering sensors are distributed sensors that take advantage of Rayleigh scattering, and time domain refractometry to create a fully distributed sensor over the length of the fibre, capable of measuring strain and temperature. Alahbabi et al. (2004), have shown development of this sensor technology to distributed sensing over distances of $6.3 \mathrm{~km}$, with spatial resolution near $1.3 \mathrm{~m}$, strain resolution of 80 micro-strain, and temperature resolution of 3 degrees centigrade. These sensors show tremendous potential for distributed sensing, but currently do not present sufficient resolution when compared to the interferometric and fibre Bragg grating point strain sensors. Although many of the interferometric strain sensors are capable of being intrinsically designed, Udd (1991) suggests that most are typically applied in an extrinsic form; thereby making them less suitable for integration into a fibre reinforced composite structure. It is for these reasons the fibre Bragg grating was selected as the optical strain sensor device with the greatest current ability for taking multiple high resolution point 
strain measurements within a composite aerospace structure, such as a helicopter rotor blade.

\subsubsection{Fibre Bragg Gratings (FBG) as Strain Sensors}

Much research has been done on the difficulties and applications of fibre Bragg gratings as strain sensors to the mechanical and aerospace sectors. Friebele et al. (1999) have presented an overview of the challenges and current solutions for embedding fibre Bragg grating sensors into spacecraft structures; tackling issues from composite fabrication techniques, ingress and egress of optical fibre, suitable source and spectral analysis components, to meeting qualification requirements for space flight.

Some research has focused on the coatings of these optical sensors. Pak (1992) examined the longitudinal shear transfer for an embedded sensor in composite materials, and concluded that a bare fibre (uncoated) would present better shear transfer than a coated fibre, unless the coating was stiffer than the core material. Uncoated optical fibre is quite fragile, and work has been done by Hadjiprocopiou et al. (1996) to optimize suitable coatings for these sensors and at the same time minimize stress concentration effects in the structure in which they are embedded. Coated optical fibre behaves more predictably when guiding light because the exact refractive index of the material surrounding the core is known. A bare fibre, when mounted, is surrounded by the epoxy, or the matrix material, which might have an unknown refractive index, or one that is inconsistent. According to Green et al. (2000), these coatings will affect both the local stress concentration of the fibre in the host material, and the strain measurement accuracy. Because of the fragile nature of bare optical fibre, ingress and egress methods 
have been developed by Kang et al. (2000) to protect the fibre from fracture at these points when dealing with sensors embedded in composite structures.

The foundation for using fibre Bragg gratings as strain sensors was laid by Bertholds et al. (1988) with the determination of the individual strain-optic coefficients for optical fibre. Since then, many researchers have applied these values in order to predict the behaviour of Bragg gratings under various strain conditions, and apply them to various engineering situations. Tian and Tao (2001) successfully showed by experiment the application of fibre Bragg gratings to determining torsional deformation of cylindrical shafts. Kim et al. (2004) successfully showed experimentally a method for determining the deflected shape of a simple beam model employing multiple fibre Bragg grating strain sensors.

\subsection{Review of Basic Fibre Optic Theory}

In what follows below in this Chapter, a brief review of the basics of fibre optic theory is given. Some insight into how fibre Bragg gratings function as intrinsic mechanical strain sensor devices for the body to which they are mounted or embedded will also be provided. "Fibre optic" generally refers to the guided transmission of light through a transparent fibre material. To understand how this fibre is able to guide light, it is essential to understand the fundamentals of "optics", the branch of physics that describes the behaviour and properties of light. 


\subsubsection{The Nature of Light}

There has been much debate throughout history over the exact nature of light, and to this day it is still not fully understood. Light was originally thought to be composed of particles, however in the 1800 's Thomas Young showed through his famous double-slit experiments that light definitely exhibited wave characteristics. Further research led James Maxwell to the conclusion that light was in fact a component of the electromagnetic wave spectrum. This theory held until the 1900 's when light interaction with materials known as semi-conductors could not be explained by the electromagnetic wave theory. This interaction, known as the photoelectric effect, was unexplained until the emergence of quantum physics. In quantum physics, energy is given a particle form, known as quanta, and when specifically dealing with light energy, these particles are referred to as photons. With quantum physics, much of the debate between the particle and wave nature of light has since been reconciled, Ghatak (1998).

When dealing with the propagation of light through any medium, it is treated as a continuum, and thus the electromagnetic (EM) wave theory is applied. The quantum physics theory is more useful when dealing with sources and detectors of light, which involves the interaction of photons with semiconductors, NEETS (2006). Since any fibre optic sensing system involves sources of light, propagation of light through a fibre optic medium, and the detection of light, both theories would need to be examined in order to formulate a complete understanding of the system. However, the scope will be limited to presenting EM wave theory only since it is critical to understanding how Bragg gratings function. 


\subsubsection{Electromagnetic Waves}

All electromagnetic waves have perpendicular electric and magnetic fields that oscillate in phase with each other and are also perpendicular to the direction of the wave propagation, as shown in figure 2.3 . "Light" commonly refers to the visible, infra-red (IR) and ultra-violet (UV) wavelengths of the electromagnetic wave spectrum. Table 2.2 shows some of the common wavelength-divided categories of electromagnetic wave spectrum.

When light encounters a substance, it is reflected, refracted, absorbed or transmitted as shown in figure 2.4. It is convenient when dealing with these interactions to think of the propagating light wave as a "ray", or simply a straight line that is travelling along the axis of the wave's propagation as previously shown in figure 2.3 . Light rays that are not refracted, transmitted, or reflected when they encounter a substance are absorbed. A substance through which almost all wavelengths of light can be transmitted is said to be transparent. There is no known substance that is perfectly transparent to all wavelengths of light; some wavelengths will always be absorbed. Silica glass is considered to be a highly transparent substance and thus is used as the core material in the majority of optical fibre. It is however not transparent to all wavelengths of light, and is thereby limited to operating within a certain range of the electromagnetic spectrum that is not absorbed.

\subsubsection{Absorption of Light in Silica Glass}

The wavelengths of light that are absorbed by silica glass are governed by quantum physics theory, the chemical makeup of the material and its crystalline atomic structure. 
A significant portion of the ultraviolet spectrum is absorbed because of its interaction with electrons, causing them to excite to higher energy states. Similarly, the infra-red spectrum is absorbed because of its interaction with the vibratory motion of the siliconoxygen atomic bonds in the silica glass, NEETS (2006). These are considered intrinsic absorptions; they are inherent to the basic chemical structure of silica glass and cannot be avoided. Intrinsic absorption limits the useful spectral range of silica glass fibre between $700 \mathrm{~nm}$ and $1600 \mathrm{~nm}$, NEETS (2006).

Besides these intrinsic absorptions, there are two other mechanisms which cause signal strength losses, extrinsic absorption and scattering. Extrinsic absorption is caused by the impurities contained within the silica fibre. The most common impurity is the hydroxyl ion $(-\mathrm{OH})$, which appears as a result of water being present in the manufacturing process of the silica fibre, and causes three absorption peaks within the $700 \mathrm{~nm}$ to $1600 \mathrm{~nm}$ range. Scattering is caused by light interacting with localised density fluctuations within the silica fibre; this results in the light being partially redirected in all directions. It is one of the primary sources of signal loss in silica optical fibre, with increasing severity towards the ultra-violet range; it is also the basis for Brillouin scattering distributed sensors.

All these forms of signal loss combined limit the operating spectral window for any system using silica glass fibre from approximately $700 \mathrm{~nm}$ to $1600 \mathrm{~nm}$, and avoiding the wavelength bands associated with the hydroxyl absorption as shown by Hecht (2006) in figure 2.5. After considering absorption of light, and the possibility of scattering, the 
remainder of light within the spectral operating window should transmit through the silica fibre with very low loss to the signal strength.

\subsubsection{Guiding Light within the Optical Fibre}

The transmitted light must now be guided within the silica fibre along the fibre axis without being lost to the exterior. This is achieved by taking advantage of the refraction and reflection of light when it encounters a boundary between two media.

When a ray of light travels in a medium other than vacuum, its rate of propagation is reduced. The ratio of the speed in a vacuum, $c$, to the speed in the medium, $v_{i}$, is known as the refractive index, $n_{i}$, of the material and is related to the relative permittivity, $\varepsilon_{r i}$, and relative permeability, $\mu_{r i}$, of the medium according to equation 2.1 .

$$
n_{i}=\frac{c}{v_{i}}=\sqrt{\varepsilon_{r i} \mu_{r i}}
$$

A ray that propagates into a medium with a higher refractive index changes angular orientation according to equation 2.2, known as Snell's Law, in order to accommodate the slower rate of wave propagation in the new medium. A ray traveling in reverse would go through an exact opposite angular change, following the same path. This reorientation of the ray is known as refraction, and is depicted in figure 2.6.

$$
n_{1} \sin \left(\theta_{1}\right)=n_{2} \sin \left(\theta_{2}\right)
$$

A special case can occur when a ray attempts to change into a faster medium at a large angle. Should the angle be such that according to Snell's Law the refracted angle would be 90 degrees, the ray would travel along the boundary of the two media. This is 
known as the critical angle, shown in figure 2.6 , and is given by equation 2.3 . For any angle of incidence larger than this value, the light does not refract, but is totally reflected from the boundary and remains within the original medium. This phenomenon is known as total internal reflection, and is the entire basis for guiding light along an optical fibre.

$$
\theta_{c r i t}=\arcsin \left(\frac{n_{2}}{n_{1}}\right)
$$

The typical cross-section of an optical fibre has a core of silica glass immediately surrounded by a cladding material of lower refractive index as shown in figure 2.7 . The simplest types of fibre are the step indexed multi-mode and step indexed single-mode, in which the entire core has the same refractive index, and the cladding has a lower index, thereby producing a step in refractive index at the core-cladding boundary as shown in figure 2.7. Light that repeatedly undergoes total internal reflection at the core-cladding boundary of the fibre is thus guided along the fibre axis as shown in figure 2.8 .

\subsubsection{Propagation of Guided Light}

Light rays of a particular wavelength that are guided along a step indexed multi-mode fibre may take one of several paths as shown in figure 2.8 due to the variation of acceptable angles for the total internal reflection criteria. The shortest ray path is straight down the central axis of the fibre, whereas the longest ray path that the light may take corresponds to the critical angle. Because these paths can differ in length, the result is that for any given wavelength of light, some of it will take longer to reach the opposite side of the optical fibre. This is known as modal dispersion, and broadens a light pulse in the time domain as it is being transmitted through a fibre. 
The different rates of propagation of light down the fibre can be represented using modal theory, in which the light propagating down the fibre is said to be a linear combination of discrete modes. This is a typical eigenvalue problem which relates the vibration of light from side to side of the fibre at a particular incident angle, thus creating a particular wave-front interference pattern, to standing wave mode shapes in the transverse direction as shown in figure 2.9, NEETS (2006). For different wavelengths of light, these mode shapes obviously occur at different ray angles because the interference patterns produced at the same angle would be different. Because the critical angle is the same for all wavelengths of light, as the wavelength increases, the number of modes that are propagated in the fibre for that wavelength decreases. Modes that are successfully propagated are said to be bound modes, and are a function of the diameter of the optical fibre, as well as the critical angle with the cladding. The lowest order standing wave is referred to as the fundamental mode, and is always propagated for all wavelengths of light in all fibre diameters. A single mode fibre has a very small diameter, and is designed to only propagate the fundamental mode, while a multi-mode fibre has a larger diameter, and is thus able to propagate many modes, NEETS (2006).

\subsection{Bragg Gratings}

A Bragg grating, also called a reflection or short-period grating, is simply a periodic perturbation to the refractive index created in the core of a section of optical fibre. It acts as an intrinsic device to filter a particular narrow wavelength band of light from the transmission spectrum, causing it to reflect backwards while allowing all other wavelengths of light to be transmitted as shown in figure 2.11 . 


\subsubsection{How Bragg Gratings are Written into Optical Fibre}

Bragg gratings are formed by exposing the core of a photosensitive fibre to an intense optical interference pattern. According to Atkins (1996), this photosensitivity can be improved for any germano-silicate fibre by using a high temperature hydrogen treatment.

Bragg gratings were first formed in optical fibre by the internal writing technique by Hill et al. (1978). They found that by creating a strong standing wave pattern of light within a germania-doped silica fibre, a grating pattern would gradually form along the full length of the fibre due to the migration of dopant particles within the fibre core. The formation of these gratings is a diffusion process driven by the interference pattern of light. When exposed to elevated temperatures, the dopant particles will diffuse out of the grating pattern into the fibre core. The grating is thus only semi-stable and will naturally degrade and fade over time. Meltz et al. (1989) showed that by controlling the intersecting angle of two ultraviolet beams through the side of the fibre, Bragg gratings could be created to reflect any desired wavelength of light. This method is known as the transverse holographic method.

A different method of forming gratings, known as the phase mask technique, shown in figure 2.10 , has since shown tremendous advantage over both these techniques when writing Bragg gratings in silica fibre. A phase mask is created from a flat piece of silica glass, and has a corrugated pattern etched onto one of the surfaces. When UV light is passed through this phase mask, it is diffracted by the corrugated pattern. Hill et al. (1997) describe how controlling the depth of the corrugation in the phase mask, significant suppression of the UV light diffracted into the zero-order is achieved. The 
result is an interference pattern created by the UV light diffracted into the $+1 /-1$ orders. The optical fibre is placed nearly in contact with the corrugated surface of the phase mask where this interference pattern is created, and causes the formation of the Bragg grating whose period is half that of the phase mask's corrugated period.

One of the major drawbacks to this technique is that a different phase mask is required for writing each particular Bragg wavelength, and the corrugation depth required to suppress the zero-order diffraction of the incident light is associated with a particular UV wavelength. Caucheteur et al. (2004) have shown that by introducing a tilt angle to the grating with respect to the fibre axis, a single phase mask can be used to produce reflection peaks at a variety of Bragg wavelengths. However, it is important to note that when using this method, the transmitted spectrum from a tilted grating, also known as a blazed grating, will lose power from the core at several discrete wavelengths of light below the Bragg wavelength into the cladding. This phenomenon introduced by the grating tilt, known as mode coupling between the core and cladding modes has been presented by Erdogan (1997), and in more detail by Laffont et al. (2001), and has found practical application to macro-bending sensors by Baek et al. (2002).

\subsubsection{Bragg Wavelength of a Grating}

The period of a Bragg grating, also known as the grating pitch, refers to the spacing or period of the perturbations to the refractive index created in the fibre as shown in figure 2.11 ; it is denoted by $\Lambda$. This periodic perturbation causes a narrow band of the incident light centered at a specific wavelength to be reflected. This specific wavelength is known 
as the Bragg wavelength, $\lambda_{B}$, or resonance wavelength of a Bragg grating. The Bragg wavelength is related to the grating pitch, $\Lambda$, and the fibre core's effective refractive index, $n_{e}$, by equation 2.4 , Kersey et al. (1997). All other unabsorbed wavelengths of light are allowed to transmit unaffected through the grating.

$$
\lambda_{B}=2 n_{e} \Lambda
$$

Because the Bragg grating affects only a particular narrow wavelength band of light, multiple Bragg gratings can be assembled into a single fibre optic line, as long as their target wavelength bands are carefully selected to not overlap, as shown by figure 2.12 . This is known as wavelength division multiplexing (WDM), and it has been shown that it is possible to fabricate a single optical fibre with nearly 100 sensors on it. This is one of the key advantages, along with the intrinsic sensing, that has made the fibre Bragg grating appealing for use in networks or arrays of sensors integrated into structures.

Any changes to the fibre that cause either a change to the grating pitch or the effective index of the fibre will result in a change to the Bragg wavelength. This phenomenon is what allows the Bragg grating to act as an intrinsic strain or temperature sensor device.

\subsection{Measuring Mechanical Strain with Bragg Gratings}

Both temperature and strain will affect the change in Bragg wavelength, Hill et al. (1997). This change in Bragg wavelength, $\Delta \lambda_{B}$, is related by equation 2.5 to the refractive index $n_{\text {core }}$, the strain-optic coefficients $P_{i j}$, the Poisson's ratio $v_{s}$, the 
coefficient of thermal expansion $\alpha_{s}$, and the thermo-optic coefficient $\zeta_{s}$ of the fibre core, to the axial strain $\varepsilon_{a x}$ and the change in temperature $\Delta T$ acting on the grating.

$$
\Delta \lambda_{B}=2 n_{e} \Lambda\left(\left\{1-\left(\frac{n_{\text {core }}^{2}}{2}\right)\left[P_{12}-v_{s}\left(P_{11}+P_{12}\right)\right]\right\} \varepsilon_{a x}+\left[\alpha_{s}+\zeta_{s}\right] \Delta T\right)
$$

\subsubsection{Strain Response of a Bragg Grating}

Assuming that temperature is maintained at a constant value, the applied axial strain causes the period of the grating to elongate or contract, and also causes changes to the fibre effective index through the photo-elastic effect, thereby changing the corresponding Bragg wavelength according to equation 2.6, Hill et al. (1997).

$$
\frac{\Delta \lambda_{B}}{\lambda_{B}}=\left\{1-\left(\frac{n_{\text {core }}^{2}}{2}\right)\left[P_{12}-v_{s}\left(P_{11}+P_{12}\right)\right]\right\} \varepsilon_{a x}
$$

All of the coefficients are combined into a single effective coefficient $P_{e}$ for a particular fibre type and wavelength. Equation 2.6 thus reduces to a simpler form, as given by equation 2.7 , Hill et al. (1997).

$$
\frac{\Delta \lambda_{B}}{\lambda_{B}}=\left\{1-P_{e}\right\} \varepsilon_{a x}
$$

Bertholds (1988) determined by experiment the strain-optic coefficients for silica fibre to be $P_{11}=0.113 \pm 0.005$ and $P_{12}=0.252 \pm 0.005$ respectively, with $n_{\text {core }}=1.458$ and $v_{s}=0.16 \pm 0.01$. Applying these numerical values into equation 2.7 produces a numerical value for $P_{e} \cong 0.205$, resulting in an optical gauge factor of 0.795 . Kersey et 
al. (1997) have reported a similar equation as shown by equation 2.8 , but with a gauge factor of 0.78 .

$$
\frac{\Delta \lambda_{B}}{\lambda_{B}}=0.78 \varepsilon_{a x}
$$

\subsubsection{Thermal Response of a Bragg Grating}

Assuming that strain is maintained at a constant value, the applied temperature change causes the thermal expansion of the fibre material, and a change to the fibre effective index, resulting in a change in the Bragg wavelength according to equation 2.9. The coefficient of thermal expansion and thermo-optic coefficient at room temperature are given by Magne et al. (1997) to be $5 \times 10^{-7} K^{-1}$ and $7 \times 10^{-6} K^{-1}$ respectively. Applying these numerical values, the thermal sensitivity of a Bragg grating is given by equation 2.10

$$
\begin{gathered}
\frac{\Delta \lambda_{B}}{\lambda_{B}}=\left[\alpha_{s}+\zeta_{s}\right] \Delta T \\
\frac{\Delta \lambda_{B}}{\lambda_{B}}=7.5 \times 10^{-6} \Delta T(K)
\end{gathered}
$$

However, Hill et al. (1997) found the values for the thermo-optic coefficient to be slightly non-linear over the temperature range as shown by figure 2.13 , and would need to be adjusted accordingly when changing temperature ranges. It is also important to note that when a Bragg grating is mounted onto a structure, the difference in thermal expansion coefficients of that structure to that of silica glass fibre may result in an additional axial strain component acting on the gauge, and would change this reduced 
thermal response equation accordingly. Magne et al. (1997) addresses this issue and uses the example of aluminum, having a coefficient of thermal expansion of $23 \times 10^{-6} \mathrm{~K}^{-1}$, resulting in an amplified thermal response of the grating given by equation 2.11 .

$$
\frac{\Delta \lambda_{B}}{\lambda_{B}}=\left[\alpha_{s}+\zeta_{s}+\left(1-p_{e}\right) \times\left(\alpha_{\text {srructure }}-\alpha_{s}\right)\right] \Delta T=25 \times 10^{-6} \Delta T(K)
$$

\subsubsection{Separation of Strain and Temperature Response}

When temperature and strain changes occur simultaneously, it is important to be able to distinguish the contribution each makes to the shift in Bragg wavelength. There are several processes by which temperature and strain effects can be separated.

The 'dummy' gauge technique is common to classical strain measurement, and employs an additional gauge to measure the temperature response independent of strain. This method, although apparently simple in principle, requires isolating a single sensor from any strain effects while allowing it to be exposed to the same temperature. It may be achieved quite easily for surface mounted gauges, or gauges embedded in large civil structures; however, the task becomes more difficult when dealing with embedded sensors and WDM arrays in fibre reinforced composites.

Some creative techniques unique to the Bragg grating behaviour have been developed to allow separation of the two measurands without these so-called 'dummy' gauges. One such technique presented by Caucheteur et al. (2004) involves writing two gratings of significantly different Bragg wavelengths into the fibre either directly beside each other (known as collocated), or over the same location (known as dual overwritten). The strain response of the two gratings will be different by one factor corresponding to equations 
2.6, 2.7 or 2.8 , and the thermal response will be different by another factor corresponding to equations $2.9,2.10$ or 2.11 . Because they are subject to the exact same strain and temperature fields, knowing how each will respond to strain and temperature independently allows the separation according to equation 2.12 , as long as the determinant of the $K$ matrix is non-zero.

$$
\left(\begin{array}{l}
\Delta \lambda_{B 1} \\
\Delta \lambda_{B 2}
\end{array}\right)=\left(\begin{array}{ll}
K_{\varepsilon 1} & K_{T 1} \\
K_{\varepsilon 2} & K_{T 2}
\end{array}\right)\left(\begin{array}{l}
\varepsilon \\
T
\end{array}\right)
$$

\subsubsection{Non-Uniform Strains and Gradients}

When tracking only the Bragg wavelength of a grating, otherwise known to be the centre wavelength of the reflected band of light from a grating, the strain value obtained is the average strain over that gratings gauge length. Peters et al. (2001) have noted that nonuniform strain fields acting over a fibre Bragg grating, such as a strain gradient, distort the reflected band of light as though each perturbation in the grating was acting separately, and reflecting the Bragg wavelength corresponding to the localized state of strain. This behaviour allows fibre Bragg gratings to not only give information about the average strain over its gauge length, but also intra-grating strain distributions.

Dong et al. (2001) have shown by experiment that mounting a Bragg grating diagonally on the side of a cantilever beam traversing the neutral axis with half the grating on each side, results in a near zero shift of the centre-wavelength, but a severe widening of the spectral peak, known as a chirping effect. This occurs because the single grating is in a non-uniform strain field, and it can be reasoned to behave as though it were two separate gratings, one mounted above the neutral axis measuring tension, and the 
other below, measuring an equal value in compression. Any change to the centre wavelength in this case would arise due to thermal affects on the structure and the grating. This intra-grating strain information is a clear advantage over the electrical resistance strain measurement devices which return only a single scalar value.

\subsubsection{Components of Strain}

Once the temperature effects have been removed from the measurement of the Bragg grating, the task still remains to relate that measured strain to the principal strains of the structure to which it is mounted or embedded. Sirkis (1993) has shown that due to the optical isotropy of silica glass, a Bragg grating is unaffected by any shear strain applied to it. This behaviour is similar to classical foil strain gauges, and allows use of the same theory for construction of a Mohr's circle to define the full state of strain in the structure.

\subsection{Concluding Remarks}

Among the numerous types of fibre optic strain sensors, fibre Bragg gratings were determined to have the greatest potential to act as embedded point strain sensors within a composite laminate structure. Their key advantages over other optical sensors are noted to be their all fibre intrinsic nature, making them less intrusive to the host structure and their ability to be highly multiplexed into sensor networks. With the theoretical strainoptic relationship of fibre Bragg gratings revised, a series of controlled experimental tests was designed to evaluate their point strain sensing abilities. This is discussed in the next Chapter. 
Table 2.1 Types of fibre optic sensors

\begin{tabular}{lll}
\hline Sensor Type & Measurands & Classification \\
\hline Fluorescence & Temperature, Viscosity, Chemical & Extrinsic \\
Reflection or Transmission & Pressure, Flow, Damage & Extrinsic \\
$\begin{array}{l}\text { Distributed Rayleigh } \\
\text { (Brillouin Scattering) }\end{array}$ & Strain, Temperature, Refractive Index & Intrinsic \\
Distributed Mode Coupling & Strain, Pressure, Temperature & Intrinsic \\
Micro-bend & Strain, Pressure, Vibration & Intrinsic \\
& $\begin{array}{l}\text { Rotation, Acceleration, Acoustics, } \\
\text { Interferometric (many types) }\end{array}$ & $\begin{array}{l}\text { Magnetic/Electric field, Strain, } \\
\text { Temperature, Pressure, Current }\end{array}$ \\
Bragg Grating & Strain, Pressure, Temperature & Intrinsic \\
Blackbody Sensors & Temperature & Intrinsic \\
\hline
\end{tabular}

Table 2.2 Typical categories of the electromagnetic spectrum

\begin{tabular}{lll}
\hline Category Name & Wavelength Range & Frequency Range (Hz) \\
\hline Gamma Rays & $<10 \mathrm{pm}$ & $10^{24}-10^{20}$ \\
X-Rays & $10 \mathrm{pm}-1 \mathrm{~nm}$ & $10^{17}-10^{20}$ \\
Ultraviolet (UV) & $1 \mathrm{~nm}-400 \mathrm{~nm}$ & $10^{15}-10^{17}$ \\
Visible Spectrum & $400 \mathrm{~nm}-750 \mathrm{~nm}$ & $4 \times 10^{14}-7.5 \times 10^{14}$ \\
Infrared (IR) & $750 \mathrm{~nm}-25 \mathrm{um}$ & $10^{13}-4 \times 10^{14}$ \\
Microwaves & $25 \mathrm{um}-1 \mathrm{~mm}$ & $3 \times 10^{11}-10^{13}$ \\
Radio Waves & $>1 \mathrm{~mm}$ & $<3 \times 10^{11}$ \\
\hline
\end{tabular}




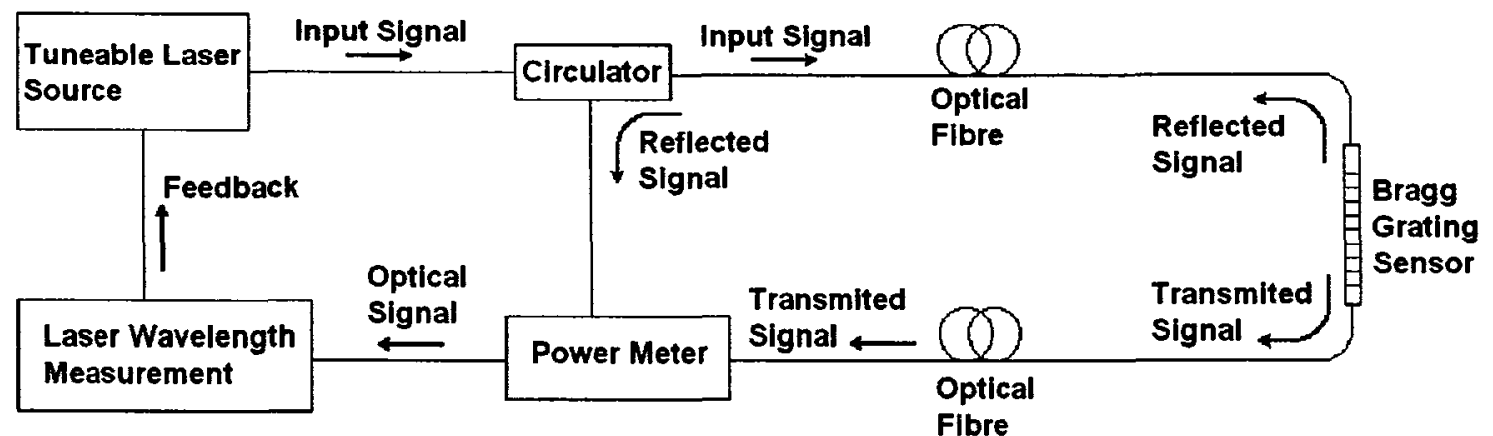

Figure 2.1 Typical optical circuit using a tuneable laser source

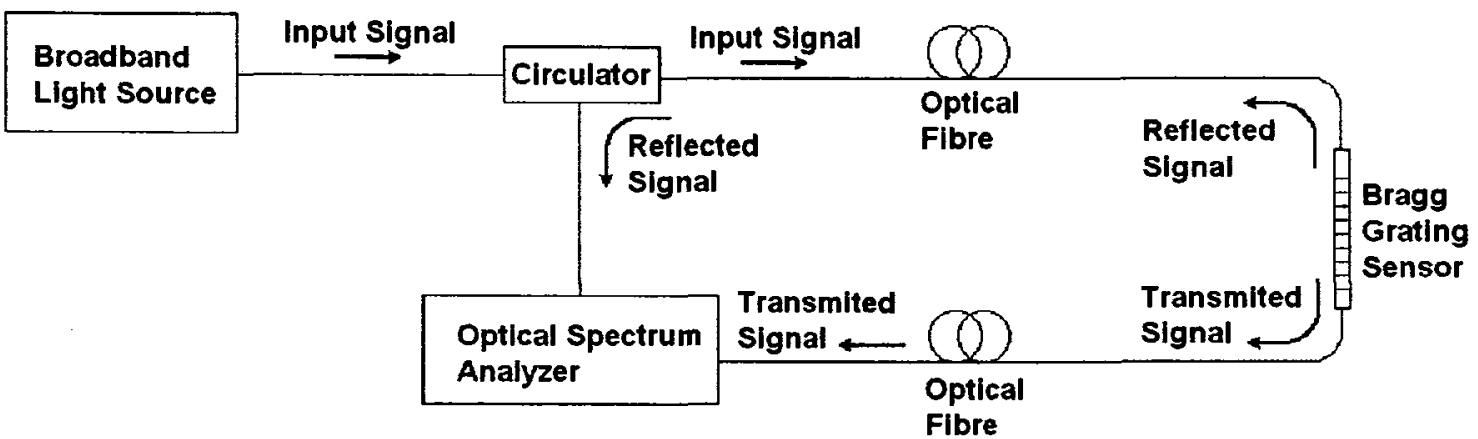

Figure 2.2 Typical optical circuit using a broadband source 


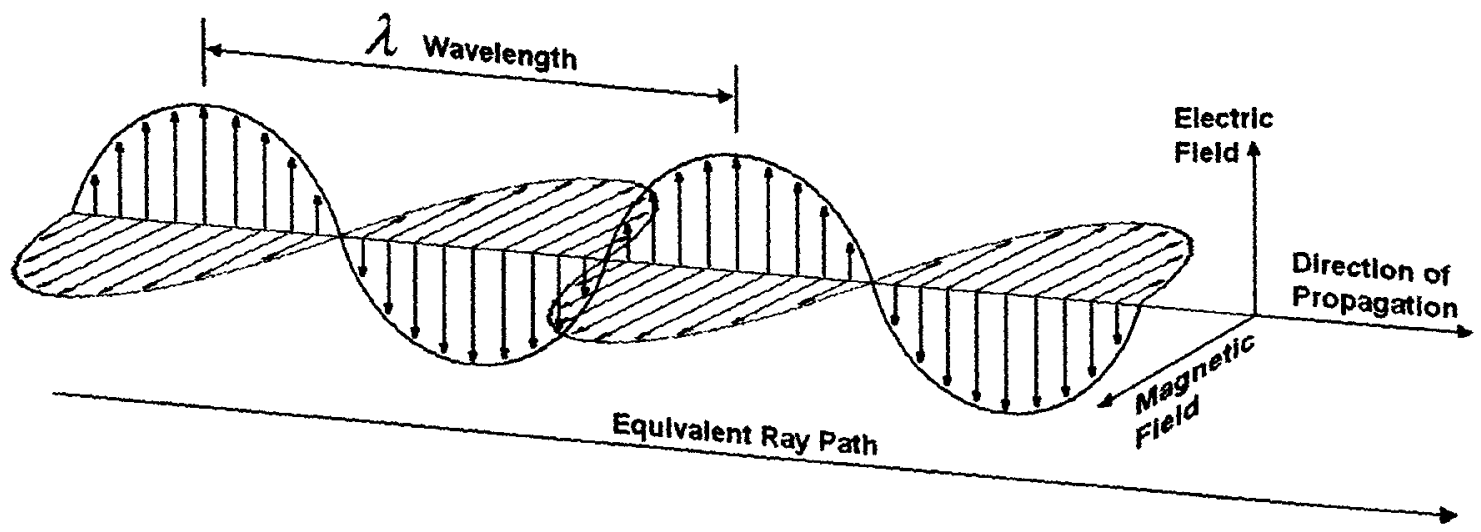

Figure 2.3 Propagation of an electromagnetic wave



Figure 2.4 Interaction of light with a substance 


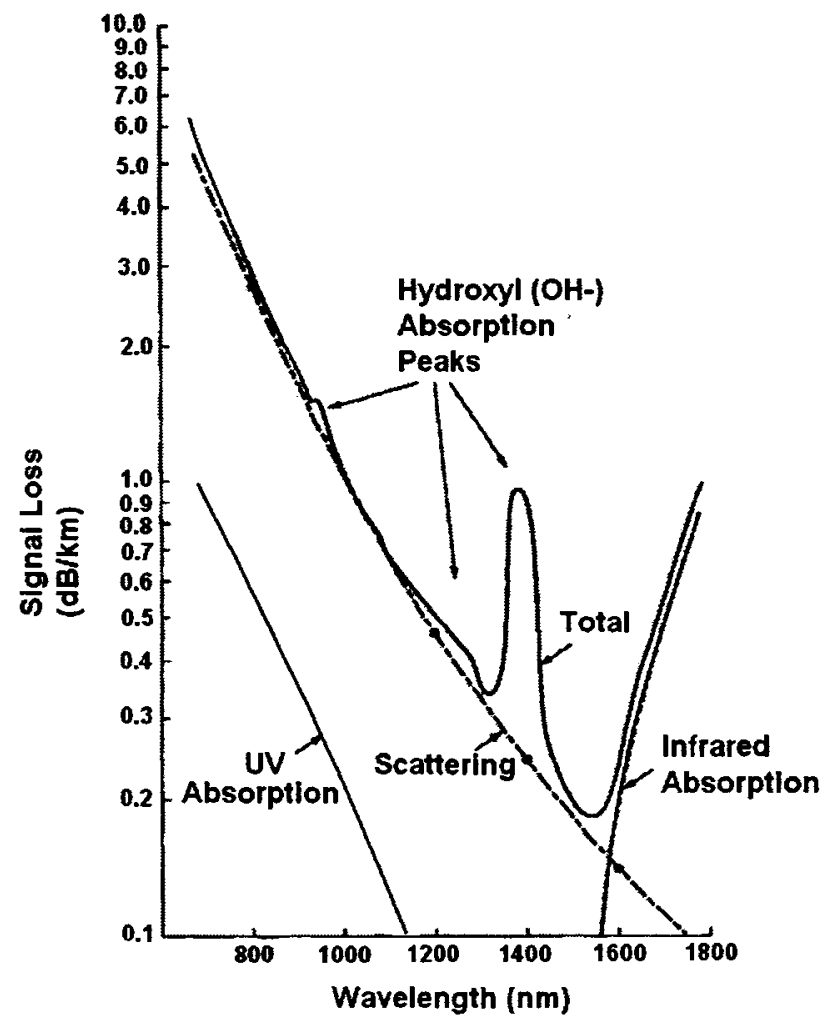

Figure 2.5 Signal attenuation of silica optical fibre - Hecht (2006)

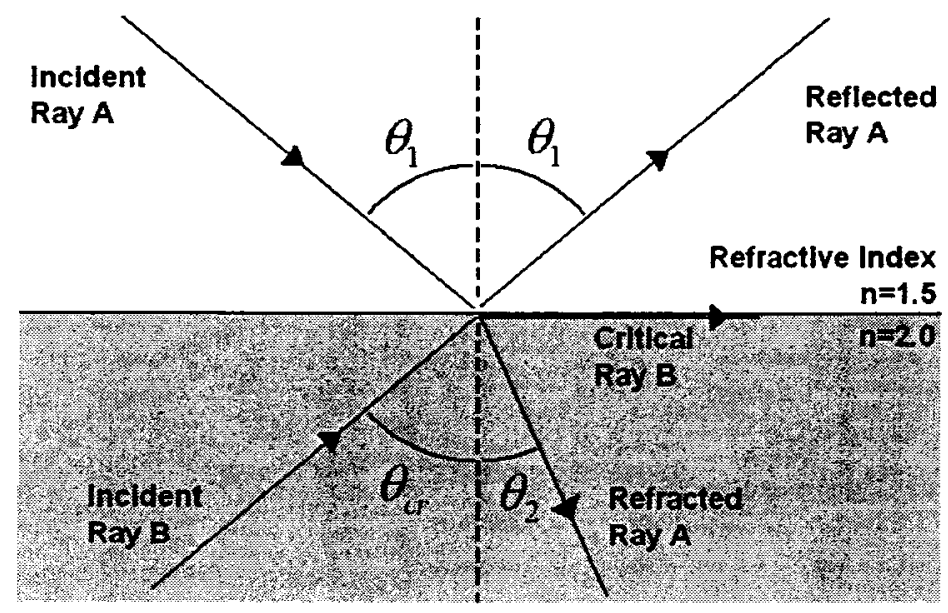

Figure 2.6 Reflection, refraction and critical angle for total internal reflection 


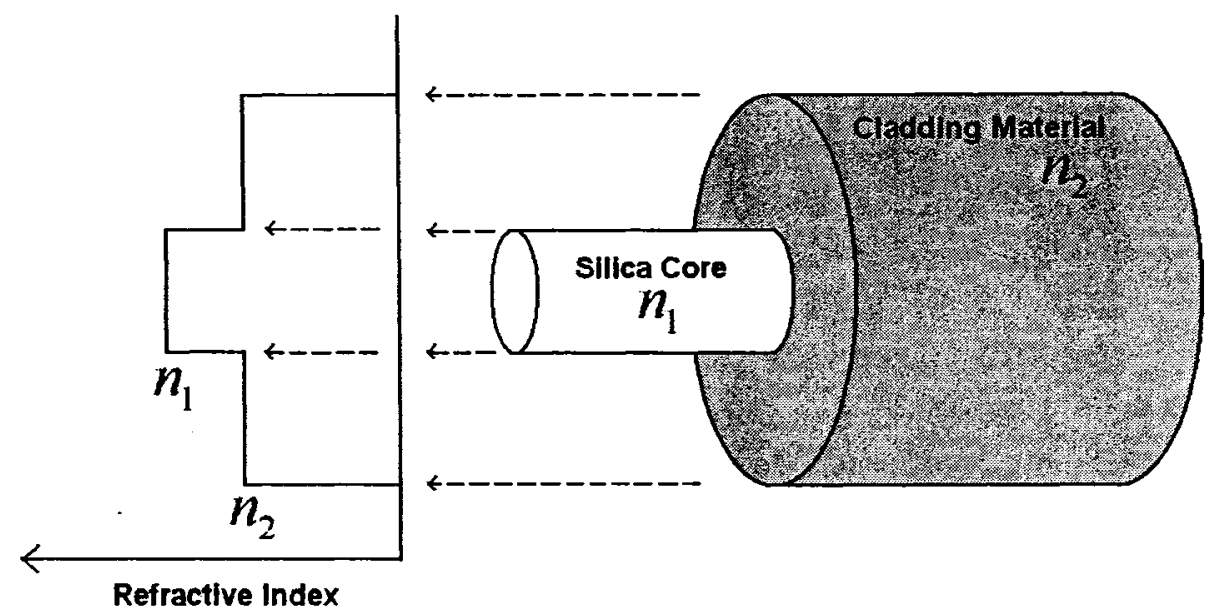

Figure 2.7 Typical cross-section of a step-indexed multi-mode silica optical fibre

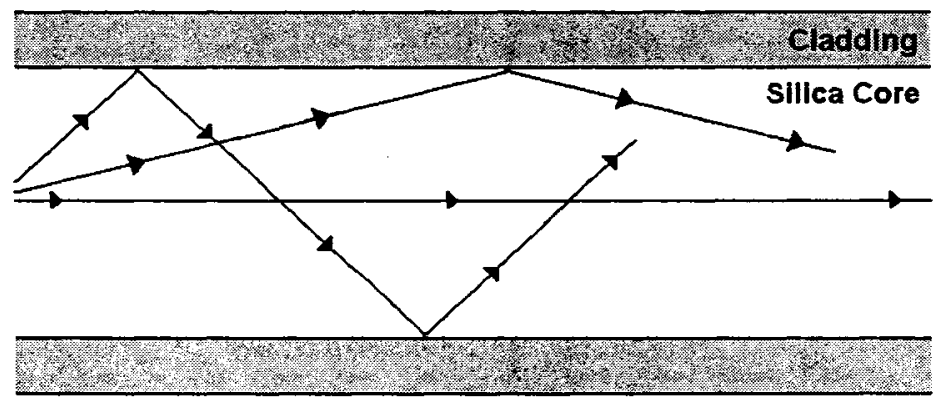

Figure 2.8 Different acceptable propagation paths of guided rays in an optical fibre 


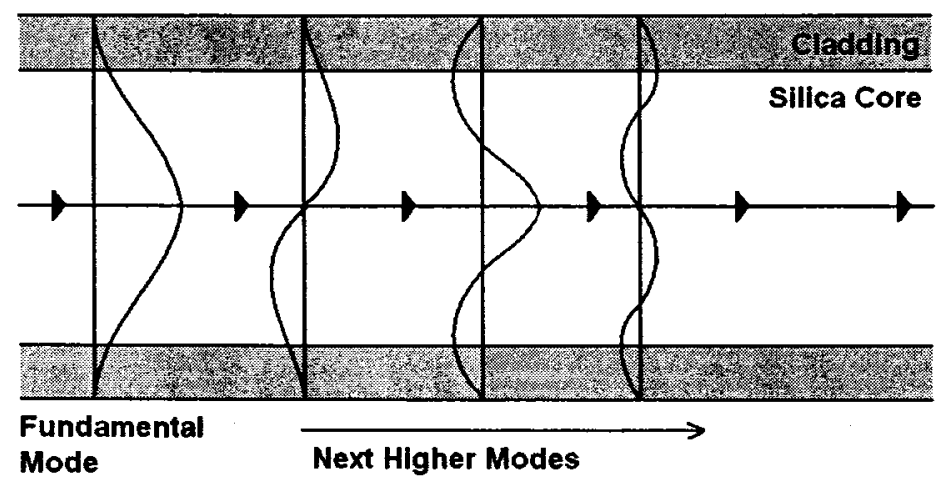

Figure 2.9 Modal representation of guided light in an optical fibre

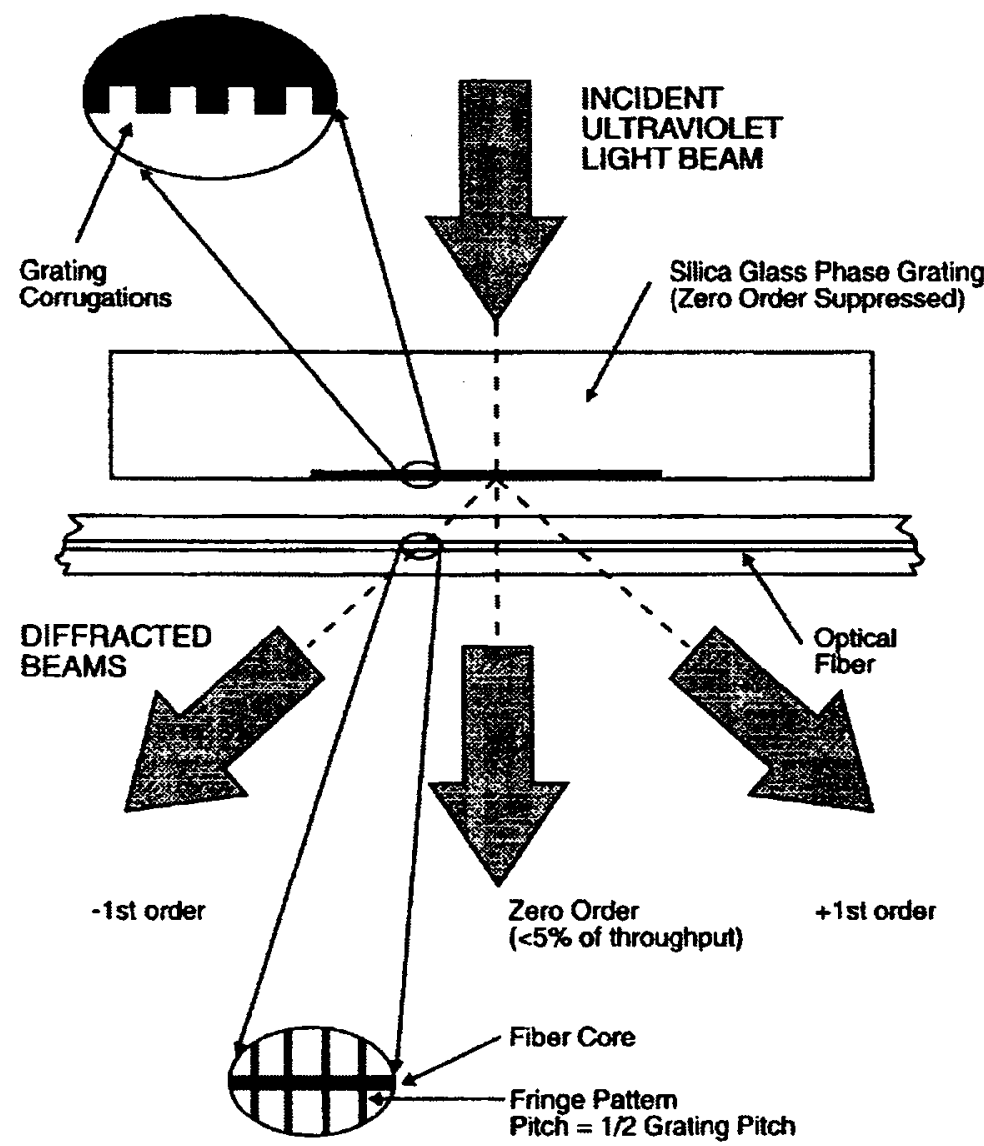

Figure 2.10 Phase mask fabrication technique - Hill et al. (1997) 




Figure 2.11 Fibre Bragg grating

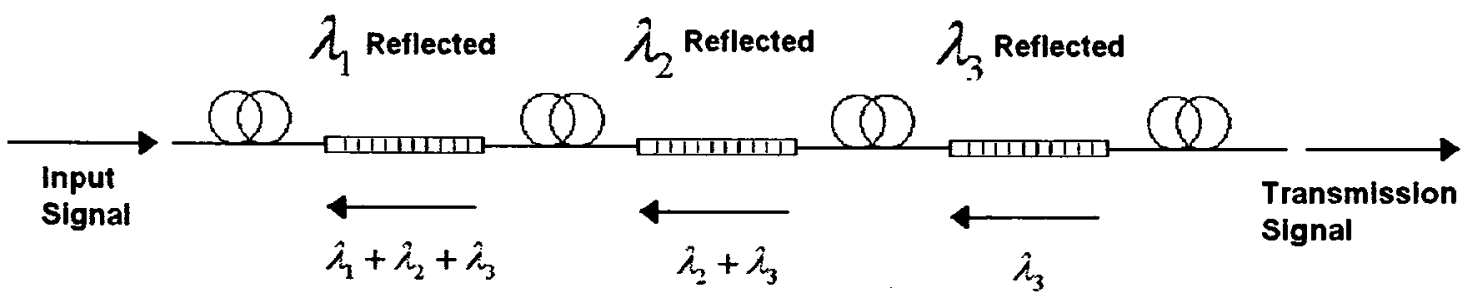

Reflection Signals

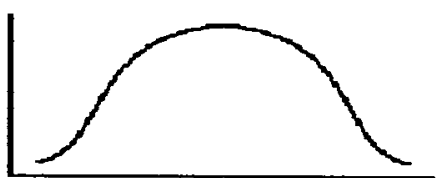

Input Signal

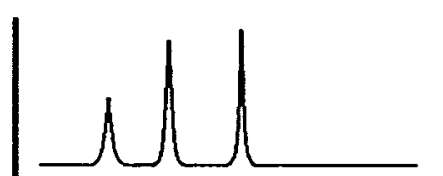

Total Reflection Signal

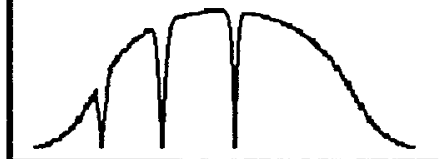

Total Transmitted Signal

Figure 2.12 Wavelength division multiplexed array of Bragg grating sensors 


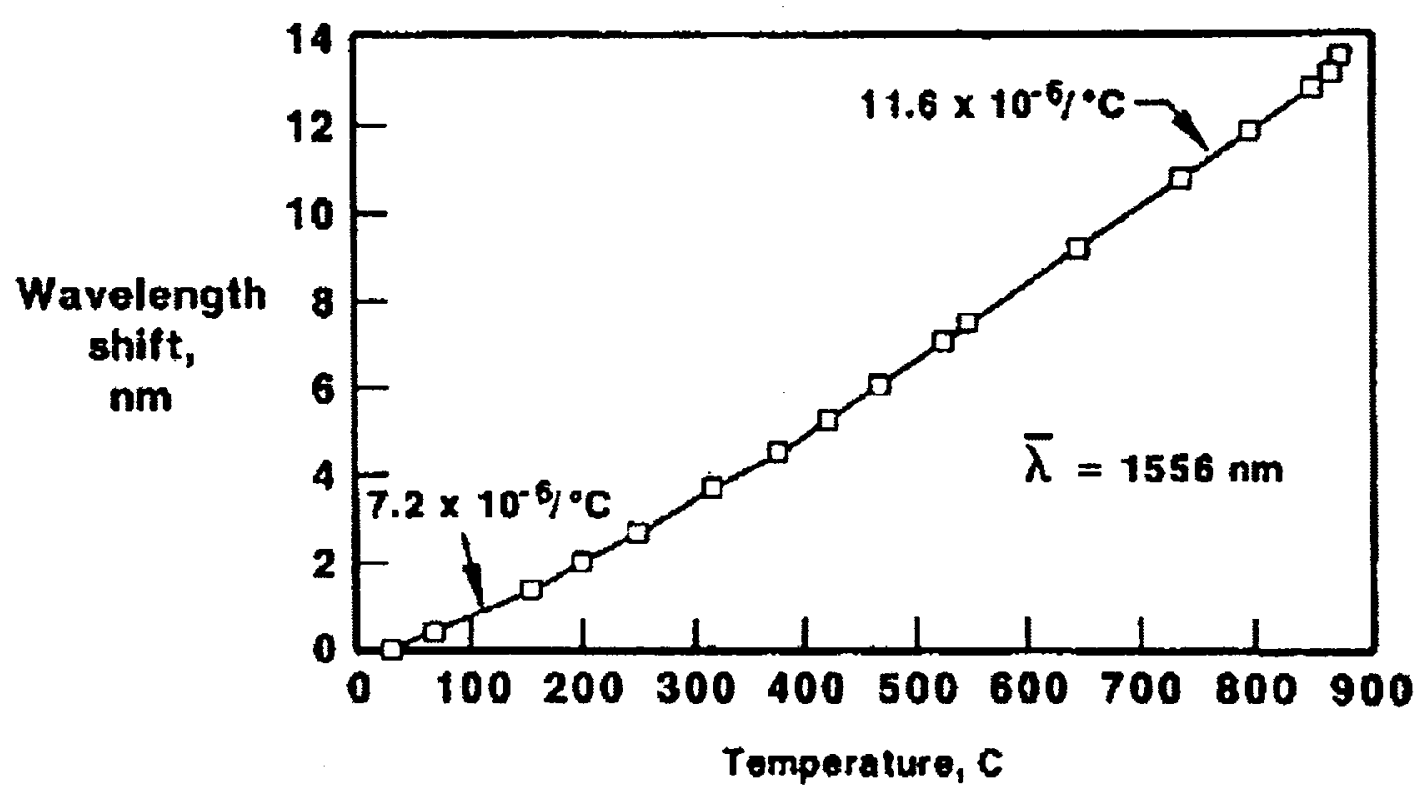

Figure 2.13 Variation of thermo-optic coefficient for silica fibre - Hill et al. (1997) 


\section{CHAPTER 3: EXPERIMENTAL SETUP}

\subsection{Introduction}

In order to test fibre Bragg gratings as point strain sensors experimentally, they must be mounted onto a structure and have a predictable strain field applied to them. To this end, a simple experimental apparatus with a cantilever beam capable of producing a range of repeatable and predictable strain fields is employed. A detailed description of the test apparatus, the optical circuit used for interrogating the fibre Bragg gratings and the use of conventional electrical resistance strain gauge rosettes is given in this Chapter. Two cantilever test beams are employed; one is an extruded aluminum beam and the other, a pultruded fibreglass beam. Analytical solutions are then presented for the strains acting on each specimen where the FBG sensors will be mounted. Finally, the analytical solutions are further verified with use of electrical resistance strain gauge rosettes.

\subsection{Equipment Setup and Integration}

The complete experimental setup involves three distinct sets of equipment integrated into a single experiment; optical, electrical, and mechanical. The optical equipment refers to the fibre Bragg gratings and the optical circuit devices required for their interrogation. The electrical equipment refers to the electrical resistance strain gauge rosettes and the 
electrical circuit devices required for their interrogation. The mechanical equipment refers to the support frame, the applied loads, and the cantilever test specimens.

\subsubsection{Optical Equipment}

The optical equipment used for interrogating fibre Bragg gratings during this work was only available for use in the Carleton Laboratory for Laser Induced Photonic Structures (CLLIPS), hereby referred to as the CLLIPS laboratory, in the Department of Electronics, Carleton University, because it is shared by multiple research projects. This required that all the mechanical equipment, and electrical resistance strain gauge equipment be relocated to the CLLIPS laboratory in order to perform the experimental tests.

The optical circuit used to interrogate a single fibre Bragg grating is shown in figure 3.1, and is similar to the tuneable laser source circuit. Most of the components in the circuit shown in figure 3.1 are a part of the SWS-OMNI system made by JDS UNIPHASE. The figure shows the optical connection of the components; the SWSOMNI system is also connected to a PC and is controlled by the SWS-OMNI 2 (version 10.0) software. The SWS-OMNI system is capable of sweeping the electromagnetic spectrum from $1519.5 \mathrm{~nm}$ to $1634 \mathrm{~nm}$ at a speed of $40 \mathrm{~nm} / \mathrm{s}$ with an absolute wavelength resolution of $2 \mathrm{pm}$, and dynamic range of $70 \mathrm{~dB}$. When measuring transmitted or reflected signals, the software normalizes the measured signal against a reference signal from the optical source. This results in measuring the relative loss in signal power, and thus corrects for any variations of intensity between wavelengths due to the source itself.

The exact values for optical signal power loss through the individual circuit components and connections are of little consequence because the parameter of interest is 
the change in Bragg wavelength of the sensor. Each sweep or scan of the optical signal produces a data set which contains values of insertion loss, a measure of the optical signal strength, at discrete wavelength increments of approximately $2.95 \mathrm{pm}$. The entire data set produces an image of the measured optical signal as shown in figure 3.2. An individual data set was collected and saved to disk for every applied load for each FBG sensor. Processing of this raw optical signal data is further described in Section 4.3.

Each of the fibre Bragg gratings used during the experimental tests was manufactured in-house by the technician in the CLLIPS laboratory using the phase mask writing technique into germanium-doped silica glass optical fibre. Details regarding the individual sensors, their properties and optical signals are presented in Chapter 4.

\subsubsection{Electrical Resistance Strain Gauge Equipment}

When a wire-grid type electrical resistance strain gauge, or foil, shown in figure 3.3 is mounted on a surface, it is sufficiently thin that it acts as a strain witness to that surface. The in-plane strains acting on the gauge cause a change in the wire length of the grid pattern, and thus its total resistance. Only strains in the axial and transverse directions to the principal axis of the gauge have an effect on the resistance; it is insensitive to shear strain because the rectangular pattern of the wire grid results in a net zero resistance change under shear deformation.

The total change in resistance $\Delta R$ is related to the axial strain $\varepsilon_{a}$ and the transverse strain $\varepsilon_{t}$ according to equation 3.1, where $R_{o}$ is the original resistance, $F_{a}$ is the axial 
strain sensitivity factor and $F_{t}$ is transverse strain sensitivity factor of the gauge, Hetenyi (1950).

$$
\frac{\Delta R}{R_{o}}=F_{a} \varepsilon_{a}+F_{t} \varepsilon_{t}
$$

When interrogating a single strain gauge, it is not possible to separate the axial and transverse strain components, unless the relationship between the two is already known, such as the Poisson contraction that occurs during simple axial tension. It is convenient therefore to define an 'apparent strain' $\varepsilon^{\prime}$ as shown in equation 3.2 , that is related to the resistance change of the gauge by what is known as the gauge factor $F$, Hetenyi (1950).

$$
\varepsilon^{\prime}=\frac{\Delta R}{R_{o}} \frac{1}{F}=\varepsilon_{a} \frac{1}{(1+k)}+\varepsilon_{t} \frac{k}{(1+k)}
$$

The gauge factor is related to the axial and transverse strain sensitivity factors by equation 3.3 and a correction factor $k$ is also defined according to equation 3.4 , which is known as the transverse sensitivity of the gauge, Hetenyi (1950).

$$
\begin{aligned}
& F=F_{a}+F_{t} \\
& k=\frac{F_{t}}{F_{a}}
\end{aligned}
$$

When multiple gauge measurements are taken simultaneously at different orientations, this correction factor can be applied to the 'apparent' strains to separate the strain components, as long as $F_{a} \neq F_{t}$. Section 3.6 describes in further detail the separation of the strain components by using multiple strain gauges. 
The gauge factor and transverse sensitivity parameters are typically provided by the gauge manufacturer. Equation 3.1 can also be represented using the gauge factor and transverse sensitivity parameters instead of the axial and transverse strain sensitivity factors.

$$
\frac{\Delta R}{R_{o}}=\varepsilon_{a} \frac{F}{(1+k)}+\varepsilon_{t} \frac{F k}{(1+k)}
$$

The change in resistance is measured by using a quarter Wheatstone bridge circuit; an electrical circuit containing four balanced resistors, one of which is the strain gauge as shown in figure 3.4. When the resistances in the circuit are balanced, the voltage across the centre of the Wheatstone bridge is zero; changes in the resistance of the strain gauge cause changes in this voltage which is measured using a highly accurate voltage measurement device. The voltage change $\Delta V$ is related to the resistance change of the gauge by equation 3.5 , where $V_{o}$ is the voltage applied to the circuit and $F_{b}$ is the bridge factor; for a quarter Wheatstone bridge circuit $F_{b}=0.25$.

$$
\frac{\Delta V}{V_{o}}=F_{b} \frac{\Delta R}{R_{o}}
$$

The strain gauge is connected to the quarter Wheatstone bridge circuit using a three lead wire configuration as shown in figure 3.5. Thermal effects can cause the resistances of these lead wires to change; however, if all the lead wires are of equal length and gauge thickness, and thus resistance, and they are exposed to the same environment, each would exhibit approximately the same change in resistance. The three wire configuration takes advantage of this situation by connecting the third wire into the opposing arm of the 
bridge circuit, thus restoring the balance of the circuit should any change in lead wire resistance occur.

The voltage source applied to the circuit, the voltage measurement device, and the other three resistors in the Wheatstone bridge circuit, known as completion resistors, are internal components to a strain gauge indicator unit shown in figure 3.5. This unit also possesses a variable resistance, which is used to balance the bridge circuit to a measured voltage of zero. The unit allows input of the gauge factor and consequently calculates the apparent strain directly from the measured voltage, which is displayed digitally in micro-

strain. A single indicator unit can easily be used to interrogate multiple strain gauges by employing a switch and balance unit, to which each gauge is connected and balanced individually in the three wire configuration. A portable strain indicator unit and a portable switch and balance unit, shown in figure 3.6, were employed to carry out the experimental electrical resistance strain gauge measurements as will be described in Section 3.6.

\subsubsection{Mechanical Equipment}

As an experimental strain apparatus, the cantilever beam offers many advantages. It is simple in design, affordable, and if designed properly, the same support apparatus can be used for a variety of test specimens and loading conditions. The steel support frame shown in figure 3.7 was used to support the cantilever beam specimens. A steel support plate was fabricated as shown in figure 3.8 which allows different specimens to be mounted at a range of angular orientations relative to the frame. 
Two different cantilever test specimens were fabricated to mount onto this test frame; one made of a hollow rectangular aluminum extrusion, and the other, made of a hollow rectangular fibreglass pultrusion. The geometry and constraints, application of loads, and material properties are presented below.

\subsection{Specimen Geometry and Constraints}

A hollow rectangular cross-section was chosen for both test specimen designs because the geometry is simple, approximate theoretical solutions to bending and torsion for this geometry are known, and it has flat surfaces ideal for attaching multiple adjacent sensors. Manufacturers typically provide geometric dimensions for their fabricated extrusion and pultrusion cross-sections. For each of the cross-sections used, these dimensions were verified using a set of Vernier calipers.

\subsubsection{Aluminum Specimen Geometry and Constraints}

The aluminum cantilever beam has a hollow rectangular cross-section with an exterior height of $h_{o}=50.8 \mathrm{~mm}$, an exterior width of $b_{o}=25.4 \mathrm{~mm}$ and a uniform wall thickness on all sides of $t=3.048 \mathrm{~mm}$ as shown in figure 3.9. The length of the beam from the free end to the fixed end is $L=812.8 \mathrm{~mm}$. Another geometric property of interest is the second moment of area $I_{x}$. For a hollow rectangular section, $I_{x}$ is given by equation 3.6, where $h_{i}$ and $b_{i}$ are the inner height and inner width of the rectangular cross section respectfully; the calculated value of $I_{x}$ for this section is given in figure 3.9 .

$$
I_{x}=\frac{b_{o} h_{o}^{3}}{12}-\frac{b_{i} h_{i}^{3}}{12}
$$


Another geometric property of interest is the average enclosed area of the hollow cross section $\bar{A}_{x y}$, which is used in the theoretical solution for the torsion induced shear stresses of Section 3.5.2. For a hollow rectangular section $\bar{A}_{x y}$ is given by equation 3.7, where $A_{o}$ is the area enclosed by the outer surfaces and $A_{i}$ is the area enclosed by the inner surfaces; the calculated value of $\bar{A}_{x y}$ for this section is given in figure 3.9.

$$
\bar{A}_{x y}=\frac{A_{o}+A_{i}}{2}=\frac{b_{o} h_{o}+b_{i} h_{i}}{2}
$$

The fixed end of the cantilever beam was welded to a circular aluminum base plate, which was mounted onto the steel support plate shown in figure 3.8 by four steel bolts. The free end of the aluminum beam was welded closed using a small rectangular aluminum plate that matched the exterior dimensions of the cross section. Holes were later drilled into this plate, and tapped with threads to allow application of bending and torsional loads on the cantilever beam.

\subsubsection{Fibreglass Specimen Geometry and Constraints}

The fibreglass cantilever beam has a hollow rectangular cross-section with an exterior height of $h_{o}=101.6 \mathrm{~mm}$, an exterior width of $b_{o}=50.8 \mathrm{~mm}$, a vertical wall thickness of $t_{y}=3.175 \mathrm{~mm}$, and a horizontal wall thickness of $t_{x}=6.350 \mathrm{~mm}$ as shown in figure 3.9 . The length of the beam from the free end to the fixed end is $L=749.3 \mathrm{~mm}$. Values for the second moment of area and the average enclosed area of this cross section are calculated using equations 3.6 and 3.7, respectively, and are given in figure 3.10. 
Fibreglass can not be welded; the cross-section was therefore clamped to a specially fabricated support structure at the fixed end to be as rigid as possible. Figure 3.11 shows the assembly of this support structure. A steel block was machined to fit tightly inside the fixed end of the cantilever beam and was then welded onto a large circular steel base plate, which was mounted onto the steel support plate shown in figure 3.8 by four steel bolts. Holes were drilled and tapped with threads into the steel plug, to which rectangular steel plates would be fastened using steel bolts, thereby clamping the walls of the crosssection on all sides as shown in figure 3.11. The free end of the fibreglass beam has a similar steel plug clamped into it as shown in figure 3.11. Holes were drilled into the face of this steel plug and tapped with threads to allow application of bending and torsional loads onto the cantilever beam.

\subsection{Applied Loads}

Static loads are applied at the free end of the cantilever specimens by suspending masses of known values from a hanger. The applied forces at the free end of the beams are a result of adding sequential masses to the hanger. The masses were individually weighed and converted into gravitational forces.

Transverse forces applied through the shear centre of the cross-section produce no torsion and only bending of the cantilever beam. The shear centre for a rectangular section coincides with the intersection of the two planes of symmetry, at the centre of the section. A hole was drilled and tapped with threads at the shear centre location on the plate at the free end of each specimen; loads will be applied through this point to produce pure bending. 
During the fabrication of the specimens, particular care was taken to ensure that the cross section remained oriented as precisely as possible in the vertical direction. This would ensure that the default loading through the shear centre would produce bending in only the vertical plane.

A load that is applied at a lateral distance from the shear centre can be resolved into a transverse load of the same value acting through the shear centre with the addition of a torsional moment about the elastic axis of the section. Application of loads at incremental lateral distances from the shear centre is achieved by attaching a horizontal torsion bar to the plate at the free end of each specimen as shown in figure 3.12. These torsion bars have evenly spaced holes from which the masses are suspended.

It should be noted that the torsional moment will cause a varying angular deflection of the cross section along the beam length. The transverse load components will thus vary along the beam length, causing some skew bending out of the vertical plane. The resulting lateral deflection of the beam will in turn cause the torsional moment to vary along the beam axis. This effect is known as coupling, and can have a pronounced effect on the beam if the bending or torsional deflections are appreciably large. As long as the deflections are small, coupling effects such as these can be considered negligible; thereby allowing the bending moments, torsion moments, and axial forces acting on the beam to be considered linearly independent of each other. Figure 3.12 shows how the loads are applied to the test specimens and resolved assuming this linear independence. By inverting the beams, or reversing the torsion bars, additional loads can be applied to the beams that have identical magnitudes of bending and torsion, but different signs. 


\subsubsection{Aluminum Specimen}

Table 3.1 lists the transverse force increments that are applied at the free end of the aluminum cantilever beam. The bending moments $M_{x}$ acting on the beam will increase linearly with distance $z$ from the free end according to equation 3.8, where $P_{y}$ is the applied transverse force.

$$
M_{x}=P_{y} z
$$

The position $z$ of each electrical resistance gauge is listed in Table 3.4. The torsional moments $T_{z}$ that accompany the transverse forces are determined by equation 3.9, where $x$ is the lateral distance from the shear centre to where the force $P_{y}$ is applied at the free end.

$$
T_{z}=P_{y} x
$$

The torsional moments are listed in Table 3.1 for all the applied forces and all the lateral positions $x_{i}$ of the aluminum beam indicated in figure 3.9.

\subsubsection{Fibreglass Specimen}

Table 3.2 shows the transverse force increments that are applied at the free end of the fibreglass cantilever beam. The corresponding bending and torsional moments again are given by equations 3.8 and 3.9 , respectively; the position $z$ of each electrical resistance gauge is listed in Table 3.5. 
The torsional moments that accompany the transverse forces are determined by equation 3.9. The torsional moments are listed in Table 3.2 for all the applied forces and all the lateral positions $x_{i}$ of the fibreglass beam indicated in figure 3.10.

\subsection{Material Properties of Specimens}

The materials used for construction of the cantilever test specimens, aluminum extrusion and fibreglass pultrusion, are considered to be linearly elastic materials. The fibreglass pultrusion is a polyester/glass composite; polyester is a polymeric material, which can exhibit non-linear visco-elastic behaviour at high temperatures. As long as the temperature remains well below the glass transition temperature of the polyester, it will behave similar to glass; the response will for all practical purposes be linear until ultimate failure of the material.

A fully anisotropic linearly elastic material requires 21 independent material constants to complete the stiffness and compliance matrices. An orthotropic material requires only 9 independent material constants because it has no coupling between the shear and direct components of stresses and strains. The coefficients of the compliance matrix for an orthotropic material are related to the 9 independent material constants of equation 3.10, see e.g. Tuttle (2004), such that $\frac{v_{y z}}{E_{z}}=\frac{v_{z y}}{E_{y}}, \frac{v_{x z}}{E_{z}}=\frac{v_{z x}}{E_{x}}, \frac{v_{x y}}{E_{y}}=\frac{v_{y x}}{E_{x}}$, where $E_{i}$ denotes the Young's modulus in the $\mathrm{i}$-th axis, $v_{i j}$ refers to the Poisson's ratio in the $\mathrm{i}-\mathrm{j}$ plane, and the numerical average, or tensor shear strain $\varepsilon_{y x}$ is related to the engineering shear strain by $\varepsilon_{y x}=\gamma_{y x} / 2$. 


$$
\left\{\begin{array}{l}
\varepsilon_{z z} \\
\varepsilon_{y y} \\
\varepsilon_{x x} \\
\varepsilon_{y x} \\
\varepsilon_{x z} \\
\varepsilon_{z y}
\end{array}\right\}=\left[\begin{array}{cccccc}
\frac{1}{E_{z}} & \frac{-v_{z y}}{E_{y}} & \frac{-v_{z x}}{E_{x}} & 0 & 0 & 0 \\
\frac{-v_{y z}}{E_{z}} & \frac{1}{E_{y}} & \frac{-v_{y x}}{E_{x}} & 0 & 0 & 0 \\
\frac{-v_{x z}}{E_{z}} & \frac{-v_{x y}}{E_{y}} & \frac{1}{E_{x}} & 0 & 0 & 0 \\
0 & 0 & 0 & \frac{1}{2 G_{y x}} & 0 & 0 \\
0 & 0 & 0 & 0 & \frac{1}{2 G_{z x}} & 0 \\
0 & 0 & 0 & 0 & 0 & \frac{1}{2 G_{z y}}
\end{array}\right]\left\{\begin{array}{l}
\sigma_{z z} \\
\sigma_{y y} \\
\sigma_{x x} \\
\sigma_{y x} \\
\sigma_{x z} \\
\sigma_{z y}
\end{array}\right\}
$$

A pultruded fibreglass beam is considered to be a transversely isotropic material. Transverse isotropy is a special case of the orthotropic material class when the material exhibits the same material properties irrespective of orientation within a plane and varying properties in the directions normal to that plane. A transversely isotropic material therefore requires only 5 independent material constants. The coefficients of the compliance matrix are found according to equation 3.11, Tuttle (2004), such that $\frac{v_{t z}}{E_{z}}=\frac{v_{z t}}{E_{t}}$, and shear modulus $G_{t t}=\frac{E_{t}}{2\left(1+v_{t t}\right)}$ for the transversely isotropic plane; the subscript ' $t$ ' denotes the isotropic $\mathrm{x}-\mathrm{y}$ plane. 


$$
\left\{\begin{array}{l}
\varepsilon_{z z} \\
\varepsilon_{y y} \\
\varepsilon_{x x} \\
\varepsilon_{y x} \\
\varepsilon_{x z} \\
\varepsilon_{z y}
\end{array}\right\}=\left[\begin{array}{cccccc}
\frac{1}{E_{z}} & \frac{-v_{z t}}{E_{t}} & \frac{-v_{z t}}{E_{t}} & 0 & 0 & 0 \\
\frac{-v_{t z}}{E_{z}} & \frac{1}{E_{t}} & \frac{-v_{t t}}{E_{t}} & 0 & 0 & 0 \\
\frac{-v_{t z}}{E_{z}} & \frac{-v_{t t}}{E_{t}} & \frac{1}{E_{t}} & 0 & 0 & 0 \\
0 & 0 & 0 & \frac{1}{2 G_{t t}} & 0 & 0 \\
0 & 0 & 0 & 0 & \frac{1}{2 G_{z t}} & 0 \\
0 & 0 & 0 & 0 & 0 & \frac{1}{2 G_{z t}}
\end{array}\right]\left\{\begin{array}{l}
\sigma_{z z} \\
\sigma_{y y} \\
\sigma_{x x} \\
\sigma_{y x} \\
\sigma_{x z} \\
\sigma_{z y}
\end{array}\right\}
$$

An aluminum extrusion is considered to be an isotropic material and requires only 2 independent material constants. The coefficients of the compliance matrix are found according to equation 3.12 , where the shear modulus $G=\frac{E}{2(1+v)}$ for an isotropic material.

$$
\left\{\begin{array}{l}
\varepsilon_{z z} \\
\varepsilon_{y y} \\
\varepsilon_{x x} \\
\varepsilon_{y x} \\
\varepsilon_{x z} \\
\varepsilon_{z y}
\end{array}\right\}=\left[\begin{array}{cccccc}
\frac{1}{E} & \frac{-v}{E} & \frac{-v}{E} & 0 & 0 & 0 \\
\frac{-v}{E} & \frac{1}{E} & \frac{-v}{E} & 0 & 0 & 0 \\
\frac{-v}{E} & \frac{-v}{E} & \frac{1}{E} & 0 & 0 & 0 \\
0 & 0 & 0 & \frac{1}{2 G} & 0 & 0 \\
0 & 0 & 0 & 0 & \frac{1}{2 G} & 0 \\
0 & 0 & 0 & 0 & 0 & \frac{1}{2 G}
\end{array}\right]\left\{\begin{array}{l}
\sigma_{z z} \\
\sigma_{y y} \\
\sigma_{x x} \\
\sigma_{y x} \\
\sigma_{x z} \\
\sigma_{z y}
\end{array}\right\}
$$




\subsubsection{Aluminum Properties}

The aluminum material used for the cantilever beam is $6063-\mathrm{T} 5$ extrusion. The required material constants for this aluminum are listed by the Mat-Web (2007) as $E=68.9 \mathrm{GPa}$ and $v=0.33$. The yield strength of this aluminum is listed as $\sigma_{Y}=145 \mathrm{MPa}$; all applied loads were verified to produce direct stresses in the beam well below this value or permanent deformation of the cantilever specimen will occur.

The material properties listed in Table 3.3 were verified by employing electrical resistance strain gauge rosettes on the cantilever specimen as will be discussed in Section 3.6. After verifying the geometry of the specimen and the applied load values, the strains measured by the gauges are shown to agree with the theoretical solutions for bending and torsion of Section 3.5, thus indicating that the material constants are indeed as quoted by Mat-Web (2007).

\subsubsection{Fibreglass Properties}

The fibreglass pultrusion used for the cantilever beam was manufactured by Bedford Reinforced Plastics Inc. The material constants quoted by the manufacturer are listed in Table 3.3. The material constant $v_{t t}$ was however not provided and was assumed to be 0.3 , by comparison to similar polyester fibreglass pultruded materials. These constants had been experimentally determined from several samples by the manufacturer; they should be verified however, because as noted by Mottram (2004), the values provided are not always true, and often have significant deviations from the real properties. Similar to the aluminum specimen, the material constants of the fibreglass cantilever beam can be 
verified by employing electrical resistance strain gauge rosettes. After verifying the geometry of the specimen and the applied load values, the strains measured by the gauges were found to disagree significantly with the corresponding theoretical solutions for bending and torsion; this will be further discussed in Section 3.6.

In order to resolve this discrepancy, additional experimental tests were done. The simplest was uni-axial tension of a flat specimen of the fibreglass pultrusion material. Several rectangular test coupons were fabricated from the walls of the fibreglass beam with the geometry as shown in figure 3.13 with the fibre direction being parallel to the coupon length. During this test, the strain gauges can also be tested to determine if they are properly measuring the true strain on the fibreglass specimens. The strain gauges were mounted as shown in figure 3.13, one aligned with the fibre direction and the other orthogonal to it.

The test coupons were monotonically loaded in simple tension using a hydraulic powered material testing system (MTS). The MTS machine has a calibrated load cell capable of accurately measuring the applied tensile force $P_{z}$ on the specimen during the loading cycle. The tensile stress $\sigma_{z z}$ is calculated using equation 3.13 , where $A_{x y}$ is the cross-sectional area of the test coupon.

$$
\sigma_{z z}=\frac{P_{z}}{A_{x y}}
$$

During the loading cycle, a linear variable displacement transducer (LVDT) extensometer simultaneously measured the displacement along the axis of loading between two points on the coupon surface. The initial distance between these points is 
known as the gauge length of the extensometer. The axial strain $\varepsilon_{z z}$ is calculated using equation 3.14, which divides the measured axial displacement $\Delta L_{g z}$ by the gauge length $L_{g z}$. Additional axial strain measurements were recorded at particular load intervals using the strain gauges mounted on the coupon surface.

$$
\varepsilon_{z z}=\frac{\Delta L_{g z}}{L_{g z}}
$$

Having independently measured the applied stress with the load cell of the MTS machine and the resulting strain on the test coupon with the extensometer, a stress versus strain plot was created as shown in figure 3.14 giving the Young's modulus $E_{z}=29.0$ $\mathrm{GPa}$. The strain response measured using the strain gauges is also shown in the figure; the slope of this curve gives $E_{z}=28.8 \mathrm{GPa}$. This value is in excellent agreement with that obtained using the extensometer, thus verifying that the strain gauges were indeed measuring the strain of the cantilever beam accurately. Several material samples were tested to verify continuity of the material properties within the cross-section of the cantilever specimen and to verify that the modulus value obtained is representative of the entire specimen.

Adequate material samples did not exist to experimentally determine the remaining material constants, such as the shear modulus $G_{z t}$. However, after verifying that the strain gauges are accurately measuring the surface strains, the necessary unknown material constants can be estimated with confidence by forcing the theoretical solutions for bending and torsion presented in Section 3.5 to agree with the rosette measurements 
obtained by experiment in Section 3.6. The material constants derived in this semiempirical method are listed in Table 3.3 as $E_{z}=30.2 \mathrm{GPa}, G_{z t}=4.1 \mathrm{GPa}$, and $v_{z t}=0.25$. The other Poisson's ratio $v_{t t}=0.3$ was assumed, and had no effect on the theoretical solution for this beam. The elastic modulus $E_{z}=30.2 \mathrm{GPa}$ estimated using the semi-empirical method in the fibre axis direction agrees within $2 \%$ of the modulus $E_{z}=28.8 \mathrm{GPa}$ as determined by the MTS experiment, thereby suggesting that the approach gave an acceptable level of accuracy for estimating the necessary material constants.

\subsection{Cantilever Beam Theoretical Solutions}

Analytical solutions derived from simple beam and torsion theory can be used to predict the stresses acting on each cantilever specimen and the resulting strains, using the values of the various parameters given in the previous sections. The solutions for combined bending and torsion loads can be obtained by simple superposition of the solution of the individual load cases.

\subsubsection{Bending of a Hollow Rectangular Cantilever Beam}

A transverse load applied at the free end of the cantilever beam that passes through the shear centre and the vertical principal axis of the hollow rectangular cross section results in simple bending of the beam in the vertical plane. This axial stress distribution in the beam due to pure bending is given by equation 3.15 , Roark (1975), where $I_{x}$ is the second moment of area of the cross section about the $\mathrm{x}$ axis, $M_{x}$ is the bending moment 
acting on that section at position $z$ about the $x$ axis, and $y_{o}$ is the position on the exterior surface of the cross section measured from the neutral axis of the beam in the $y$ direction.

$$
\sigma_{z z}=-\frac{M_{x} y_{0}}{I_{x}}
$$

In bending, the shear stress $\tau_{z y}$ at the top and bottom surfaces of the hollow rectangular cross section is zero, and can thus be ignored for any strain gauges or FBG sensors mounted on those surfaces.

\subsubsection{Torsion of a Thin-Walled Hollow Rectangular Section}

For a hollow thin-walled rectangular section under torsion, the average shear stress $\bar{\tau}_{x z}$ on the top surface near the middle of the wall can be found using equation 3.16, Roark (1975), where $\bar{A}_{x y}$ is the average of the area enclosed by the inner surfaces and the area enclosed by the outer surfaces given by equation $3.7, T_{z}$ is the applied torsional moment about the elastic axis, and $t_{y}$ is the thickness of the top wall.

$$
\bar{\tau}_{x z}=\frac{T_{z}}{2 \bar{A}_{x y} t_{y}}
$$

For a thin wall, the shear stress on the inner and outer walls will be very close, and is therefore assumed to be constant across the wall thickness. For slightly thicker walls, the stress will vary in an approximately linear distribution across the thickness of the wall with proportion to the distance from the elastic axis of the section. Knowing the average

shear stress $\bar{\tau}_{x z}$ in the wall, equation 3.17 can be used to determine an approximate shear 
stress $\tau_{x z o}$ on the exterior surface of the wall, where $y_{o}$ and $y_{i}$ are the positions on the outer and inner surfaces of the wall respectively; they are measured from the neutral axis in the $y$ direction.

$$
\tau_{x z o}=\bar{\tau}_{x z} \frac{2 y_{o}}{\left(y_{o}+y_{i}\right)}
$$

\subsubsection{Mohr's Circle of Strains}

The strains at a particular point are related to the applied stresses through Hooke's Law as described in Section 3.4. Rotating the coordinate axes of the strain field by an angle $\theta$ causes the components of strain to transform according to equations $3.18,3.19$ and 3.20.

$$
\begin{aligned}
& \varepsilon_{x^{\prime}}=\frac{\varepsilon_{x}+\varepsilon_{z}}{2}+\frac{\varepsilon_{x}-\varepsilon_{z}}{2} \cos 2 \theta+\varepsilon_{x z} \sin 2 \theta \\
& \varepsilon_{z^{\prime}}=\frac{\varepsilon_{x}+\varepsilon_{z}}{2}-\frac{\varepsilon_{x}-\varepsilon_{z}}{2} \cos 2 \theta-\varepsilon_{x z} \sin 2 \theta \\
& \varepsilon_{x^{\prime} z^{\prime}}=-\frac{\varepsilon_{x}-\varepsilon_{z}}{2} \sin 2 \theta+\varepsilon_{x z} \cos 2 \theta
\end{aligned}
$$

Mohr's circle, shown in figure 3.15 , is a graphical representation of this strain coordinate transformation. The centre $C$ and radius $R$ of the Mohr's circle are given by equations 3.21 and 3.22 respectively.

$$
\begin{gathered}
C=\frac{\varepsilon_{x}+\varepsilon_{z}}{2} \\
R=\sqrt{\left(\frac{\varepsilon_{x}-\varepsilon_{z}}{2}\right)^{2}+\varepsilon_{x z}{ }^{2}}
\end{gathered}
$$


The orientation of the principal planes where the shear strain components are zero is denoted by $\theta_{P}$ as shown in figure 3.16 and can easily be shown to be as given by equation 3.23.

$$
\theta_{P}=\frac{1}{2} \tan ^{-1}\left(\frac{2 \varepsilon_{x z}}{\varepsilon_{x}-\varepsilon_{z}}\right)
$$

The principal strains, $\varepsilon_{P 1}$ and $\varepsilon_{P 2}$, are thus related by equations 3.24 and 3.25 to the centre and radius of the circle respectively.

$$
\begin{aligned}
& \varepsilon_{P 1}=C+R \\
& \varepsilon_{P 2}=C-R
\end{aligned}
$$

The maximum shear strain $\varepsilon_{x z \max }$ occurs at an orientation $\theta=45^{\circ}$ from the principal orientation as shown in figure 3.16 and is related to the radius by equation 3.26 .

$$
\varepsilon_{x z \max }=R
$$

With the Mohr's circle, it is possible to determine the components of the strain field at any arbitrary orientation. This is convenient for determining the individual components of strain acting on a strain gauge that is mounted at a particular orientation.

\subsection{Strain Gauge Rosette Measurements}

An electrical resistance strain gauge rosette, shown in figure 3.17 , is a set of individual electrical resistance strain gauges that are oriented differently, but packaged together onto a single bondable strip, on which the orientations of the gauges relative to each other are known. Rosettes are a convenient way of using the strain gauges to experimentally measure the full state of strain at a given location. 
The apparent strains of each gauge on the rosette are measured using the three wire quarter bridge circuit described in Section 3.1.2. The apparent strains can then be separated into the axial and transverse strain components by applying the transverse sensitivity correction factors $k_{t 1}, k_{2}$, and $k_{t 3}$. This separation requires specific knowledge of the relative orientations of each gauge, and also requires that all gauges in that rosette are witness to the same strain components. For example, if the rosette were located on the side of a cantilever beam with some of gauges above the neutral axis and some below, they would not see the same strains. 'Stacked' rosettes are available for these instances, where the individual gauges are mounted on top of each other to have coincident centers.

The transverse strain components can be removed from the apparent strains by using equations $3.27,3.28$, and 3.29, Hetenyi (1950), where $v_{o}=0.285$ is the Poisson ratio of the material used by the manufacturer for determination of the gauge factor and transverse sensitivity parameters.

$$
\begin{gathered}
\varepsilon_{1}=\frac{\varepsilon_{1}^{\prime}\left(1-v_{o} k_{t 1}\right)-k_{t 1} \varepsilon_{3}^{\prime}\left(1-v_{o} k_{t 3}\right)}{1-k_{t 1} k_{t 3}} \\
\varepsilon_{2}=\frac{\varepsilon_{2}^{\prime}\left(1-v_{o} k_{t 2}\right)}{1-k_{t 2}}-\frac{k_{t 2}\left[\varepsilon_{1}^{\prime}\left(1-v_{o} k_{t 1}\right)\left(1-v_{o} k_{t 3}\right)+\varepsilon_{3}^{\prime}\left(1-v_{o} k_{t 3}\right)\left(1-v_{o} k_{t 1}\right)\right]}{\left(1-k_{t 1} k_{t 3}\right)\left(1-k_{t 2}\right)} \\
\varepsilon_{3}=\frac{\varepsilon_{1}^{\prime}\left(1-v_{o} k_{t 1}\right)-k_{t 1} \varepsilon_{3}^{\prime}\left(1-v_{o} k_{t 3}\right)}{1-k_{t 1} k_{t 3}}
\end{gathered}
$$

These relationships are specially derived for the rectangular rosette configuration shown in figure 3.17 ; other convenient rosette configurations, such as the delta, also shown in figure 3.17 , have similar corrective relationships specially derived. Once the 
axial strain component on each gauge in the rosette has been deciphered from the apparent strains, the values can be used to obtain the full state of strain at that point from a Mohr's circle. Figure 3.18 shows graphically how the three gauge axial strains are used to construct Mohr's circle, and equations 3.30, 3.31, and 3.32, Hetenyi (1950), analytically define the centre $C$, radius $R$, and principal orientation $\theta_{P}$ respectively.

$$
\begin{gathered}
C=\frac{\varepsilon_{1}+\varepsilon_{3}}{2} \\
R=\frac{1}{2} \sqrt{\left(\varepsilon_{1}-\varepsilon_{3}\right)^{2}+\left(2 \varepsilon_{2}-\varepsilon_{1}-\varepsilon_{3}\right)^{2}} \\
\theta=\frac{1}{2} \tan ^{-1}\left(\frac{2 \varepsilon_{2}-\varepsilon_{1}-\varepsilon_{3}}{\varepsilon_{3}-\varepsilon_{1}}\right)
\end{gathered}
$$

\subsubsection{Installation of Electrical Resistance Gauges}

Two identical rectangular rosettes were used on each specimen; one located at the top surface and the other located at the bottom surface of the beam. The individual gauge properties, gauge factors, and transverse sensitivities provided by the manufacturer are listed in Table 3.4.

The rosettes were mounted onto the surface using a cyanoacrylate adhesive following the surface preparation and installation guidelines of the manufacturer. A strip of bondable terminals was simultaneously mounted adjacent to each rosette and were electrically connected to the individual gauge terminals by soldering small magnet wires onto them. They provide a larger terminal area for attaching the gauge lead wires and protects the rosette from disbond should the lead wires be pulled. 
To verify that no damage was caused to the gauges during installation, the electrical resistance value of each gauge was verified using a multi-meter. The rosette positions were then measured from the location of applied loads as listed in Table 3.4. It was difficult to align the rosette so that one gauge lies perfectly along the principal axis of the beam. The misalignment can be calculated from experimental measurement as the principal angle during pure bending. The constant angular offset between theoretical and experimental lines shown in the principal angle plot of figure 3.21 is the true spatial orientation of the rosette with respect to the principal axes of the beam. This value is calculated for each rosette and is listed in Table 3.4.

\subsubsection{Aluminum Specimen}

The apparent strain was converted to the true strain along the axis of each gauge using the manufacturer gauge properties of Table 3.4. Using the Mohr's strain circle analysis, the principal strains, maximum shear strains, and principal angles were obtained for each rosette and compared to the corresponding theoretical values for the aluminum test specimen. These results are graphically and numerically presented for bending and combined bending and torsion in figures $3.19-3.30$ and Tables $3.5-3.6$, where figures $3.19-3.24$ and Table 3.5 correspond to the top surface rosette, and figures $3.25-3.30$ and Table 3.6 correspond to the bottom surface rosette.

The principal strains measured by the top surface rosette agree within $2 \%$ of theoretical values over the tested range, whereas the measurements obtained by the bottom surface rosette agree slightly less, but still within $3 \%$. The excellent agreement with theoretical values verifies that the test specimen behaviour follows the analytical 
solutions and that the material constants of the specimen listed by Mat-Web (2007), as shown in Table 3.3 and described in Section 3.4.2 are accurate.

\subsubsection{Fibreglass Specimen}

The same analysis of the principal strains, maximum shear strains and principal angles was carried out for the fibreglass specimen as was described for the aluminum specimen. Figures $3.31-3.32$ and Table 3.8 show that there is significant disagreement between the measured and theoretical values when using the material constants provided by the manufacturer. Additional material testing was therefore carried out as described in Section 3.4.3 to resolve this disagreement. The material testing verified that the rosettes measured accurately the surface strains of the fibreglass specimen, and that the material constants were significantly different from those given by the manufacturer.

Knowing that the rosettes were correctly measuring the strains, the remaining material constants necessary for completion of the analytical solution were estimated by forcing the theoretical values to agree with the rosette measurements. Figures $3.33-3.35$ show the original disagreement in the axial strain components measured by the rosettes in the $\alpha=0$ degree, $\alpha=45$ degree and $\alpha=90$ degree directions, respectively. Figures 3.36 3.38 show the final agreement of the $\alpha=0$ degree, $\alpha=45$ degree, and $\alpha=90$ degree axial strain components after the corrections on the material constants.

Repeating the analysis of the principal strains using the new estimated material constants listed in Table 3.3, figures 3.39 - 3.44 and Table 3.9 show the agreement of the principal strains measured by the top surface rosette with the theoretical values. The principal strains were forced to agree within $2 \%$ over the tested range in order to have a 
similar accuracy as the aluminum theoretical solution. A similar analysis was repeated using the rosette on the bottom surface of the fibreglass cantilever specimen; the results were not significantly different, and are therefore not presented here.

\subsection{Concluding Remarks}

The experimental results of Section 3.6 using the electrical resistance strain gauge rosettes have shown that the theoretical solutions of Section 3.5 accurately predicted the strain fields present at the top and bottom surfaces of both the aluminum and fibreglass cantilever beams. The apparatus is now ready for fibre Bragg grating sensors to be experimentally tested, whose measurements can then be compared to theoretical solutions and those obtained using conventional electrical resistance gauges. 
Table 3.1 Aluminum specimen applied loads

\begin{tabular}{l|llllllll}
\hline $\begin{array}{l}\text { Force } \\
(\mathbf{N})\end{array}$ & \multicolumn{7}{|l}{ Torsional Moment (Nm) } \\
\hline$X=X_{i}$ & $X=X_{0}$ & $X=X_{1}$ & $X=X_{2}$ & $X=X_{3}$ & $X=X_{4}$ & $X=X_{5}$ & $X=X_{6}$ & $X=X_{7}$ \\
\hline 0.00 & 0.00 & 0.00 & 0.00 & 0.00 & 0.00 & 0.00 & 0.00 & 0.00 \\
50.76 & 0.00 & 1.29 & 2.58 & 3.87 & 5.16 & 6.45 & 7.74 & 9.02 \\
94.41 & 0.00 & 2.40 & 4.80 & 7.19 & 9.59 & 11.99 & 14.39 & 16.79 \\
139.16 & 0.00 & 3.53 & 7.07 & 10.60 & 14.14 & 17.67 & 21.21 & 24.74 \\
183.70 & 0.00 & 4.67 & 9.33 & 14.00 & 18.66 & 23.33 & 28.00 & 32.66 \\
228.27 & 0.00 & 5.80 & 11.60 & 17.39 & 23.19 & 28.99 & 34.79 & 40.59 \\
275.09 & 0.00 & 6.99 & 13.97 & 20.96 & 27.95 & 34.94 & 41.92 & 48.91 \\
319.63 & 0.00 & 8.12 & 16.24 & 24.36 & 32.47 & 40.59 & 48.71 & 56.83 \\
363.28 & 0.00 & 9.23 & 18.45 & 27.68 & 36.91 & 46.14 & 55.36 & 64.59 \\
408.04 & 0.00 & 10.36 & 20.73 & 31.09 & 41.46 & 51.82 & 62.18 & 72.55 \\
452.57 & 0.00 & 11.50 & 22.99 & 34.49 & 45.98 & 57.48 & 68.97 & 80.47 \\
497.14 & 0.00 & 12.63 & 25.25 & 37.88 & 50.51 & 63.14 & 75.76 & 88.39 \\
\hline
\end{tabular}

Table 3.2 Fibreglass specimen applied loads

\begin{tabular}{l|llllll}
\hline $\begin{array}{l}\text { Force } \\
(\mathbf{N})\end{array}$ & \multicolumn{7}{l}{ Torsional Moment (Nm) } \\
\hline$X=X_{i}$ & $X=X_{0}$ & $X=X_{1}$ & $X=X_{2}$ & $X=X_{3}$ & $X=X_{4}$ & $X=X_{5}$ \\
\hline \hline 0.00 & 0.00 & 0.00 & 0.00 & 0.00 & 0.00 & 0.00 \\
50.76 & 0.00 & 2.58 & 5.16 & 7.74 & 10.31 & 12.89 \\
94.41 & 0.00 & 4.80 & 9.59 & 14.39 & 19.18 & 23.98 \\
139.16 & 0.00 & 7.07 & 14.14 & 21.21 & 28.28 & 35.35 \\
183.70 & 0.00 & 9.33 & 18.66 & 28.00 & 37.33 & 46.66 \\
228.27 & 0.00 & 11.60 & 23.19 & 34.79 & 46.38 & 57.98 \\
317.89 & 0.00 & 16.15 & 32.30 & 48.45 & 64.60 & 80.74 \\
407.52 & 0.00 & 20.70 & 41.40 & 62.11 & 82.81 & 103.51 \\
497.14 & 0.00 & 25.25 & 50.51 & 75.76 & 101.02 & 126.27 \\
586.77 & 0.00 & 29.81 & 59.62 & 89.42 & 119.23 & 149.04 \\
\hline
\end{tabular}


Table 3.3 Specimen material properties

\begin{tabular}{llll}
\hline Elastic Constant & $\begin{array}{l}\text { Al6063-T5 } \\
\text { (Mat-Web) }\end{array}$ & $\begin{array}{l}\text { Fibreglass } \\
\text { Manufacturer }\end{array}$ & $\begin{array}{l}\text { Fibreglass } \\
\text { Empirical }\end{array}$ \\
\hline \hline$E_{1}$ & $68.95 \mathrm{GPa}$ & $19.3 \mathrm{GPa}$ & $30.2 \mathrm{GPa}$ \\
$E_{t}$ & $68.95 \mathrm{GPa}$ & $5.5 \mathrm{GPa}$ & $\mathrm{N} / \mathrm{A}$ \\
$v_{1 t}$ & 0.33 & 0.28 & 0.25 \\
$v_{t t}$ & 0.33 & 0.28 & $\mathrm{~N} / \mathrm{A}$ \\
$G_{1 t}$ & $25.92 \mathrm{GPa}$ & $3.1 \mathrm{GPa}$ & $\mathrm{N} / \mathrm{A}$ \\
$G_{t}$ & $25.92 \mathrm{GPa}$ & $3.1 \mathrm{GPa}$ & $4.1 \mathrm{GPa}$ \\
$C T E_{1}$ & $23.4 \mu \mathrm{m} / \mathrm{m}^{\circ} \mathrm{C}$ & $8.0 \mu \mathrm{m} / \mathrm{m}^{\circ} \mathrm{C}$ & $\mathrm{N} / \mathrm{A}$ \\
$C T E_{t}$ & $23.4 \mu \mathrm{m} / \mathrm{m}^{\circ} \mathrm{C}$ & $30.0 \mu \mathrm{m} / \mathrm{m}^{\circ} \mathrm{C}$ & N/A \\
\hline
\end{tabular}

Table 3.4 Test specimen electrical resistance gauge rosette information

\begin{tabular}{llllllll}
\hline Beam & Gauge & $\begin{array}{l}\text { Rosette } \\
\text { Surface }\end{array}$ & $\begin{array}{l}\mathbf{z} \\
(\mathbf{m m})\end{array}$ & $\begin{array}{l}\text { Orientation } \\
\text { (Degrees) }\end{array}$ & $\begin{array}{l}\text { Gauge } \\
\text { Factor }\end{array}$ & $\begin{array}{l}\text { Transverse } \\
\text { Sensitivity }\end{array}$ & $\begin{array}{l}\text { Resistance } \\
\text { (Ohm) }\end{array}$ \\
\hline Al. & 1 & Top & 661.7 & $-1.32+0$ & 2.08 & 0.013 & 120 \\
Al. & 2 & Top & 661.7 & $-1.32+45$ & 2.11 & 0.009 & 120 \\
Al. & 3 & Top & 661.7 & $-1.32+90$ & 2.08 & 0.013 & 120 \\
Al. & 4 & Bottom & 661.7 & $-2.25+0$ & 2.08 & 0.013 & 120 \\
Al. & 5 & Bottom & 661.7 & $-2.25-45$ & 2.11 & 0.009 & 120 \\
Al. & 6 & Bottom & 661.7 & $-2.25-90$ & 2.08 & 0.013 & 120 \\
\hline Fg. & 1 & Top & 655.6 & $3.95+0$ & 2.08 & 0.013 & 120 \\
Fg. & 2 & Top & 655.6 & $3.95+45$ & 2.11 & 0.009 & 120 \\
Fg. & 3 & Top & 655.6 & $3.95+90$ & 2.08 & 0.013 & 120 \\
Fg. & 4 & Bottom & 655.6 & $1.27+0$ & 2.08 & 0.013 & 120 \\
Fg. & 5 & Bottom & 655.6 & $1.27+45$ & 2.11 & 0.009 & 120 \\
Fg. & 6 & Bottom & 655.6 & $1.27+90$ & 2.08 & 0.013 & 120 \\
\hline
\end{tabular}


Table 3.5 Principal strains on the aluminum specimen measured by the top rosette

\begin{tabular}{llllll}
\hline $\begin{array}{l}\text { Principal } \\
\text { Strain }\end{array}$ & \multicolumn{2}{l}{ Moment $(\mathbf{N m})$} & \multicolumn{2}{l}{ Strain (micro-strain) } & $\begin{array}{l}\text { Percent } \\
\text { Difference }\end{array}$ \\
\cline { 2 - 5 } & Bending & Torsional & Theoretical & Rosette & \\
\hline P1 & 328.94 & 0.00 & 905.85 & 893.21 & -1.40 \\
& -328.94 & 0.00 & 298.93 & 293.01 & -1.98 \\
& 328.94 & 88.39 & 966.22 & 964.87 & -0.14 \\
& -328.94 & -88.39 & 359.30 & 357.80 & -0.42 \\
\hline P2 & 328.94 & 0.00 & -298.93 & -299.74 & 0.27 \\
& -328.94 & 0.00 & -905.85 & -904.97 & -0.10 \\
& 328.94 & 88.39 & -359.30 & -364.60 & 1.47 \\
& -328.94 & -88.39 & -966.22 & -974.54 & 0.86 \\
\hline
\end{tabular}

Table 3.6 Principal strains on the aluminum specimen measured by the bottom rosette

\begin{tabular}{|c|c|c|c|c|c|}
\hline \multirow{2}{*}{$\begin{array}{l}\text { Principal } \\
\text { Strain }\end{array}$} & \multicolumn{2}{|c|}{ Moment (Nm) } & \multicolumn{2}{|c|}{ Strain (micro-strain) } & \multirow{2}{*}{$\begin{array}{l}\text { Percent } \\
\text { Difference }\end{array}$} \\
\hline & Bending & Torsional & Theoretical & Rosette & \\
\hline \multirow[t]{4}{*}{ P1 } & 328.94 & 0.00 & 298.93 & 290.91 & -2.68 \\
\hline & -328.94 & 0.00 & 905.85 & 876.99 & -3.19 \\
\hline & 328.94 & 88.39 & 360.31 & 362.90 & 0.72 \\
\hline & -328.94 & -88.39 & 967.23 & 948.75 & -1.91 \\
\hline \multirow[t]{4}{*}{$\mathrm{P} 2$} & 328.94 & 0.00 & -905.85 & -877.95 & -3.08 \\
\hline & -328.94 & 0.00 & -298.93 & -291.85 & -2.37 \\
\hline & 328.94 & 88.39 & -967.23 & -949.73 & -1.81 \\
\hline & -328.94 & -88.39 & -360.31 & -363.86 & 0.99 \\
\hline
\end{tabular}


Table 3.7 Principal strains on the fibreglass specimen in poor agreement

\begin{tabular}{llllll}
\hline $\begin{array}{l}\text { Principal } \\
\text { Strain }\end{array}$ & \multicolumn{2}{l}{ Moment (Nm) } & \multicolumn{2}{c}{ Strain (micro-strain) } & $\begin{array}{l}\text { Percent } \\
\text { Difference }\end{array}$ \\
\cline { 2 - 5 } & Bending & Torsional & Theoretical & Rosette & \\
\hline \hline P1 & 384.71 & 0.00 & 551.14 & 351.90 & -36.15 \\
& -384.71 & 0.00 & 154.32 & 89.45 & -42.03 \\
& 384.71 & 144.38 & 754.00 & 546.96 & -27.46 \\
& -384.71 & -144.38 & 357.18 & 249.53 & -30.14 \\
\hline P2 & 384.71 & 0.00 & -154.32 & -90.04 & -41.65 \\
& -384.71 & 0.00 & -551.14 & -353.26 & -35.90 \\
& 384.71 & 144.38 & -357.18 & -236.72 & -33.72 \\
& -384.71 & -144.38 & -754.00 & -561.69 & -25.51 \\
\hline
\end{tabular}

Table 3.8 Principal strains on the fibreglass specimen in good agreement

\begin{tabular}{llllll}
\hline $\begin{array}{l}\text { Principal } \\
\text { Strain }\end{array}$ & \multicolumn{2}{l}{ Moment $(\mathbf{N m})$} & \multicolumn{2}{l}{ Strain (micro-strain) } & Percent \\
\cline { 2 - 5 } & Bending & Torsional & Theoretical & Rosette & Difference \\
\hline P1 & 384.71 & 0.00 & 352.70 & 352.02 & -0.19 \\
& -384.71 & 0.00 & 89.78 & 89.48 & -0.33 \\
& 384.71 & 144.38 & 523.87 & 547.11 & 4.44 \\
& -384.71 & -144.38 & 260.95 & 249.57 & -4.36 \\
\hline P2 & 384.71 & 0.00 & -89.78 & -90.07 & 0.33 \\
& -384.71 & 0.00 & -352.70 & -353.38 & 0.19 \\
& 384.71 & 144.38 & -260.95 & -236.76 & -9.27 \\
& -384.71 & -144.38 & -523.87 & -561.84 & 7.25 \\
\hline
\end{tabular}




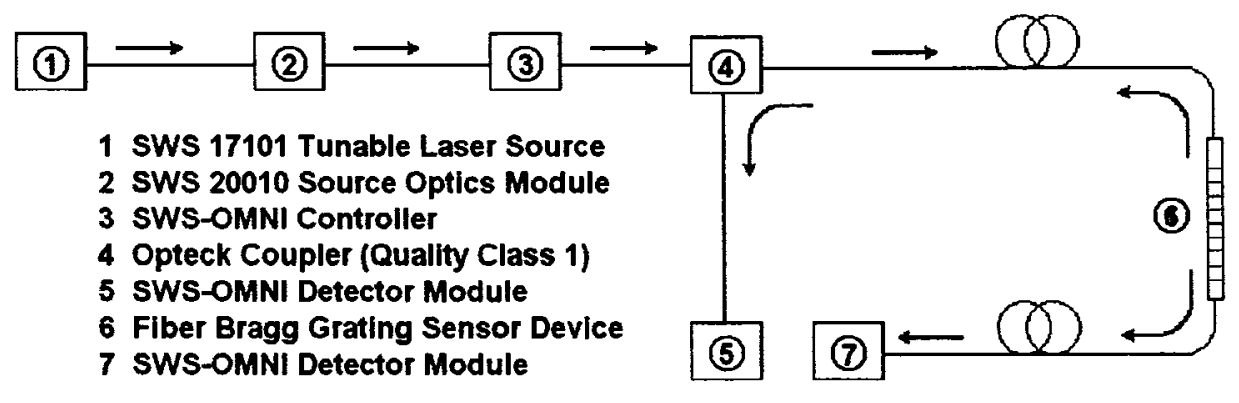

Figure 3.1 Optical interrogation circuit

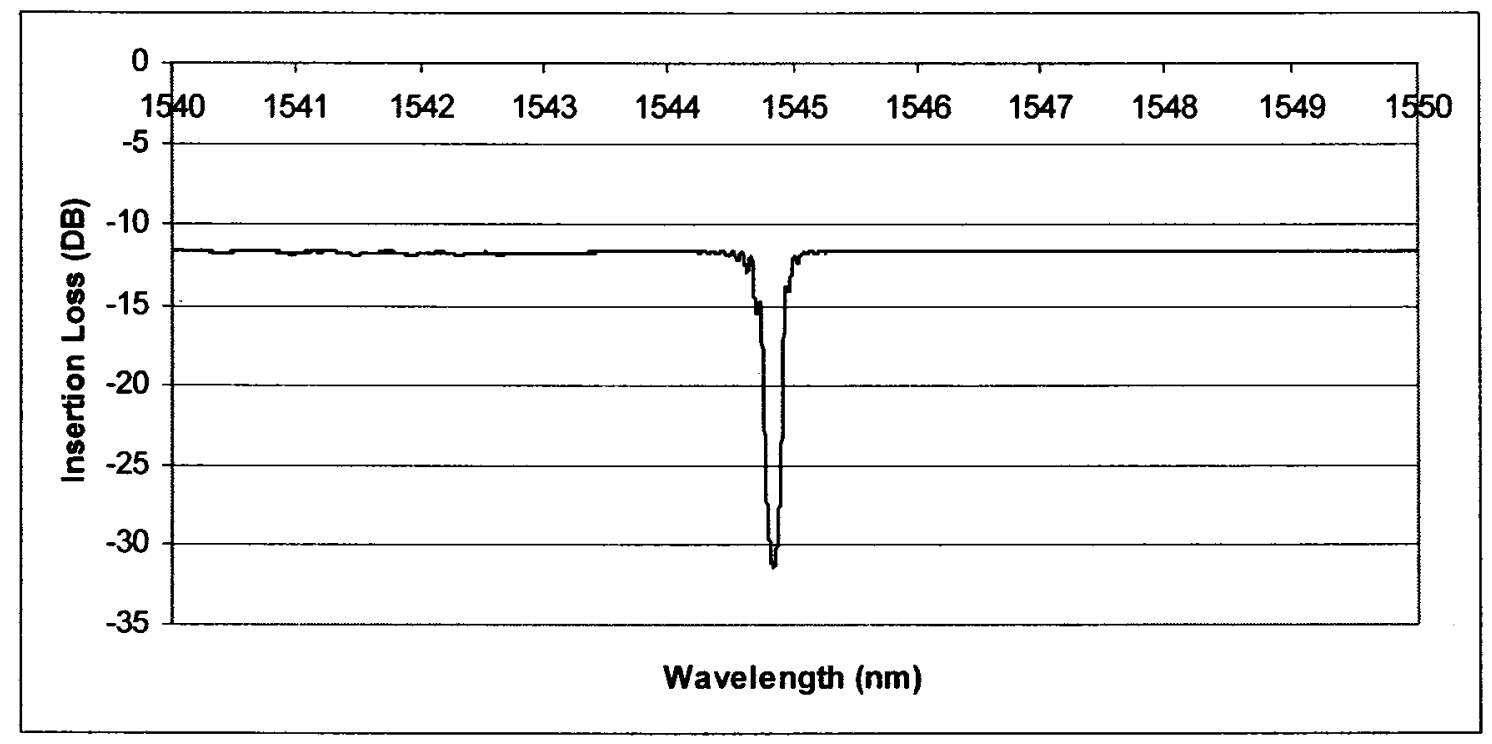

Figure 3.2 Optical signal sample of a fibre Bragg grating 




Figure 3.3 Wire grid type electrical resistance strain gauge

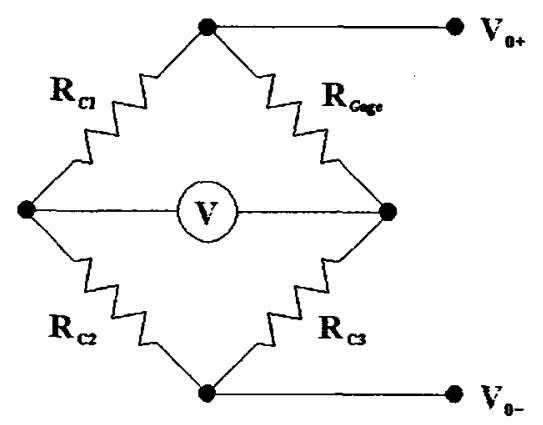

Figure 3.4 Wheatstone bridge circuit 


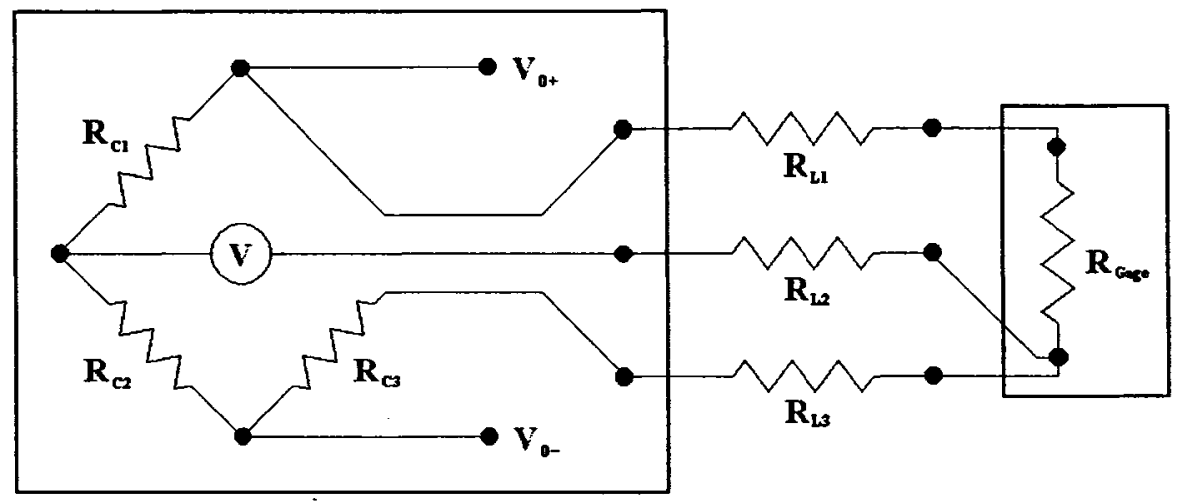

Figure 3.5 Single gauge in quarter bridge configuration

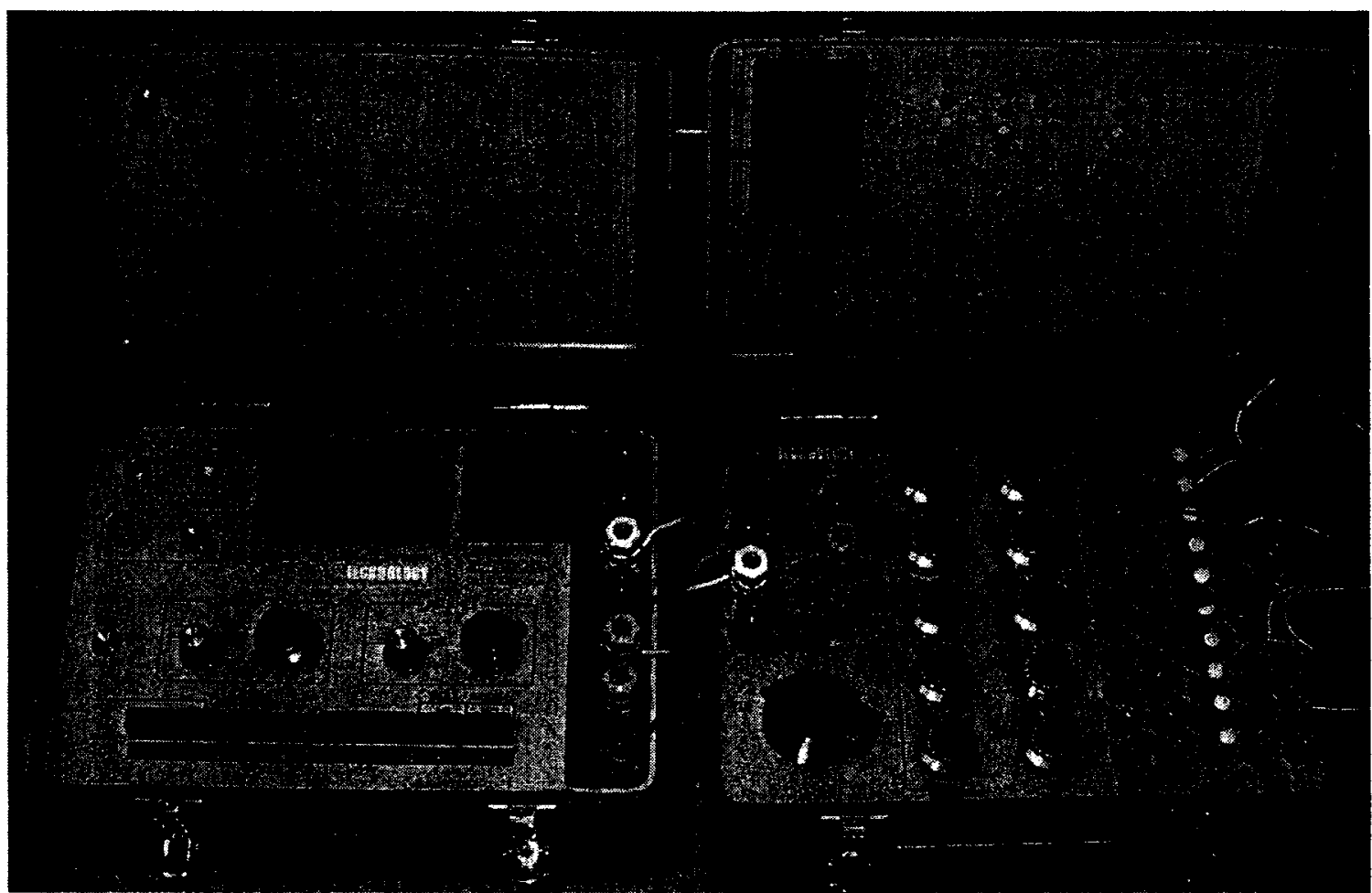

Figure 3.6 Portable strain indicator and switch and balance units 


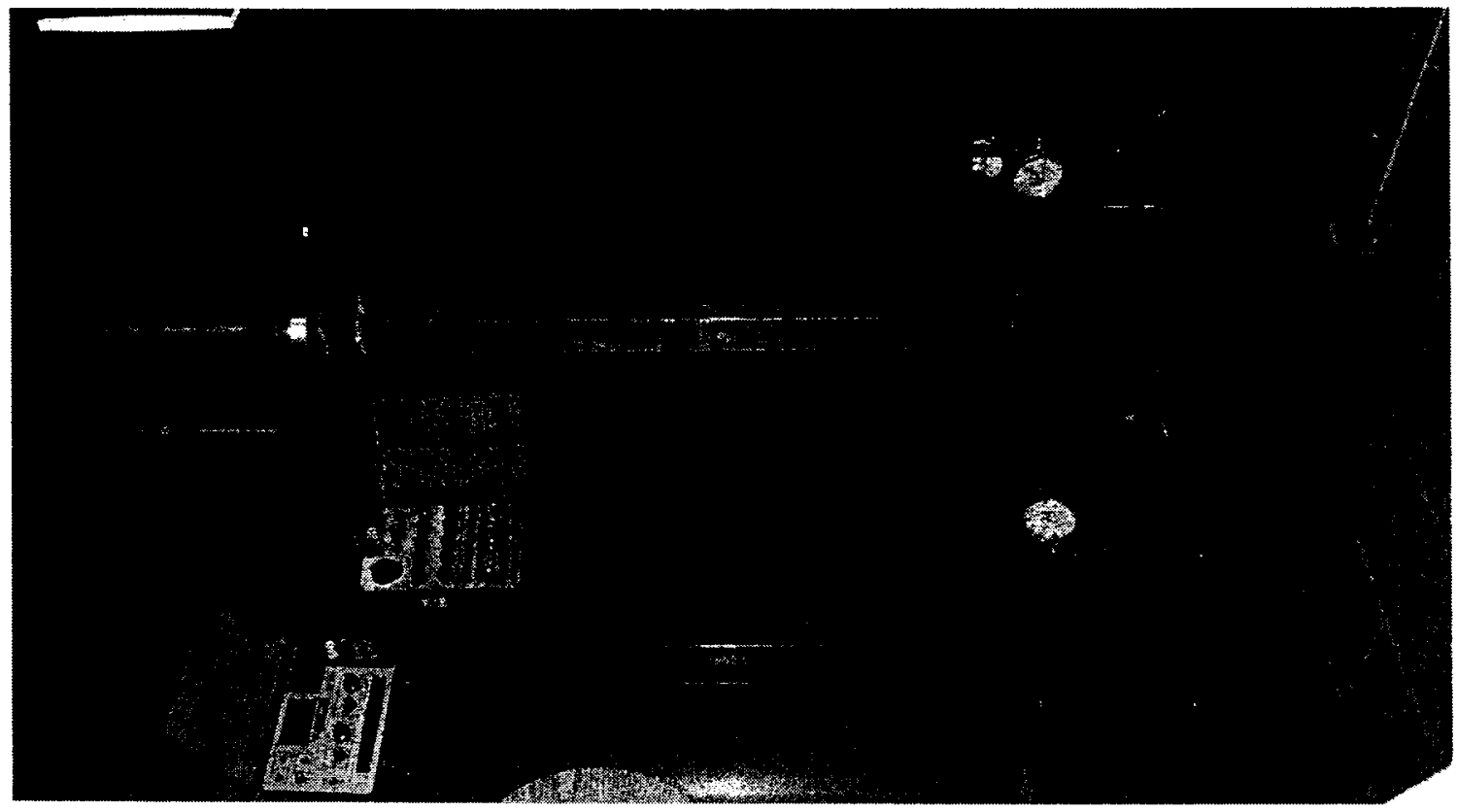

Figure 3.7 Steel support frame

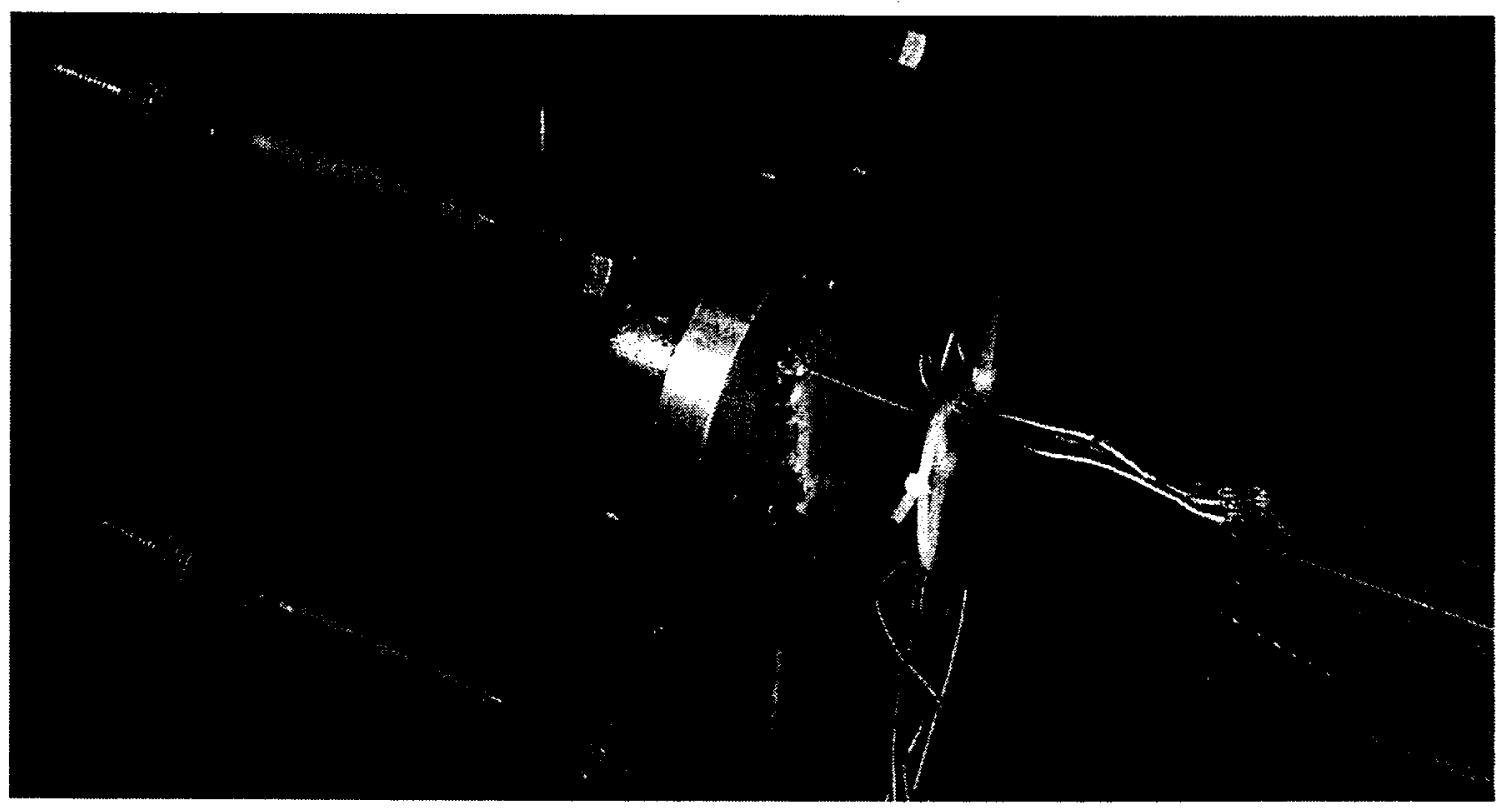

Figure 3.8 Steel support plate 


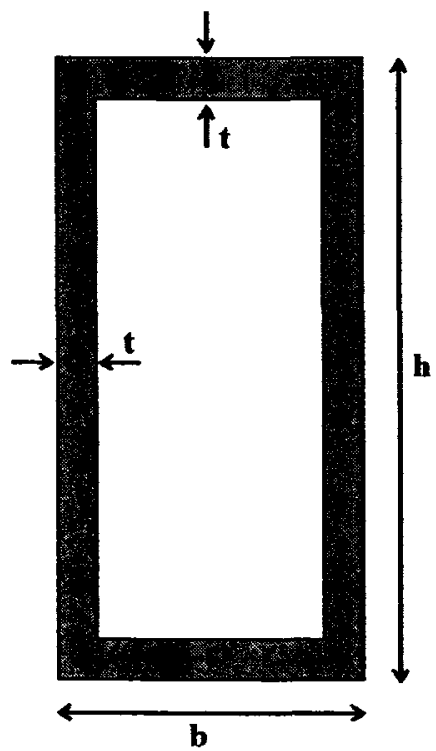

$$
\begin{aligned}
b & =25.40 \mathrm{~mm} \\
h & =50.80 \mathrm{~mm} \\
t & =3.048 \mathrm{~mm} \\
L & =812.8 \mathrm{~mm} \\
I_{x \mathbf{x}} & =133772 \mathrm{~mm}^{4} \\
\bar{A} & =1076.64 \mathrm{~mm}^{2}
\end{aligned}
$$

h

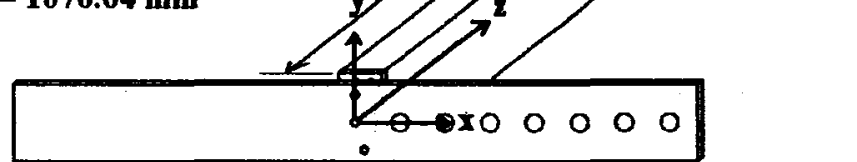

$$
\begin{aligned}
& x_{0}=0.000 \mathrm{~mm} \\
& x_{1}=25.40 \mathrm{~mm} \\
& x_{2}=50.80 \mathrm{~mm} \\
& x_{3}=76.20 \mathrm{~mm}
\end{aligned}
$$$$
x_{4}=101.6 \mathrm{~mm}
$$$$
x_{3}=127.0 \mathrm{~mm}
$$$$
x_{6}=152.4 \mathrm{~mm}
$$$$
x_{7}=177.8 \mathrm{~mm}
$$

Figure 3.9 Aluminum section: geometric properties

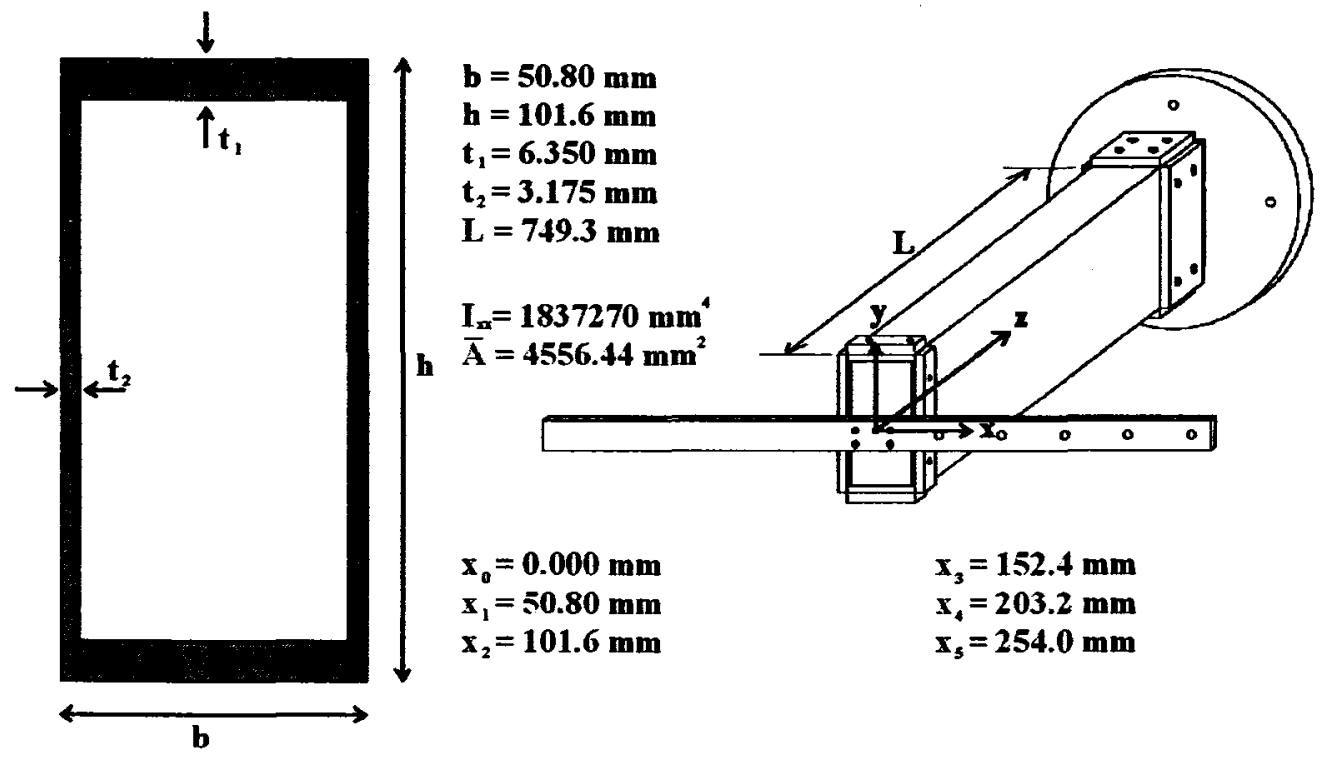

Figure 3.10 Fibreglass section: geometric properties 


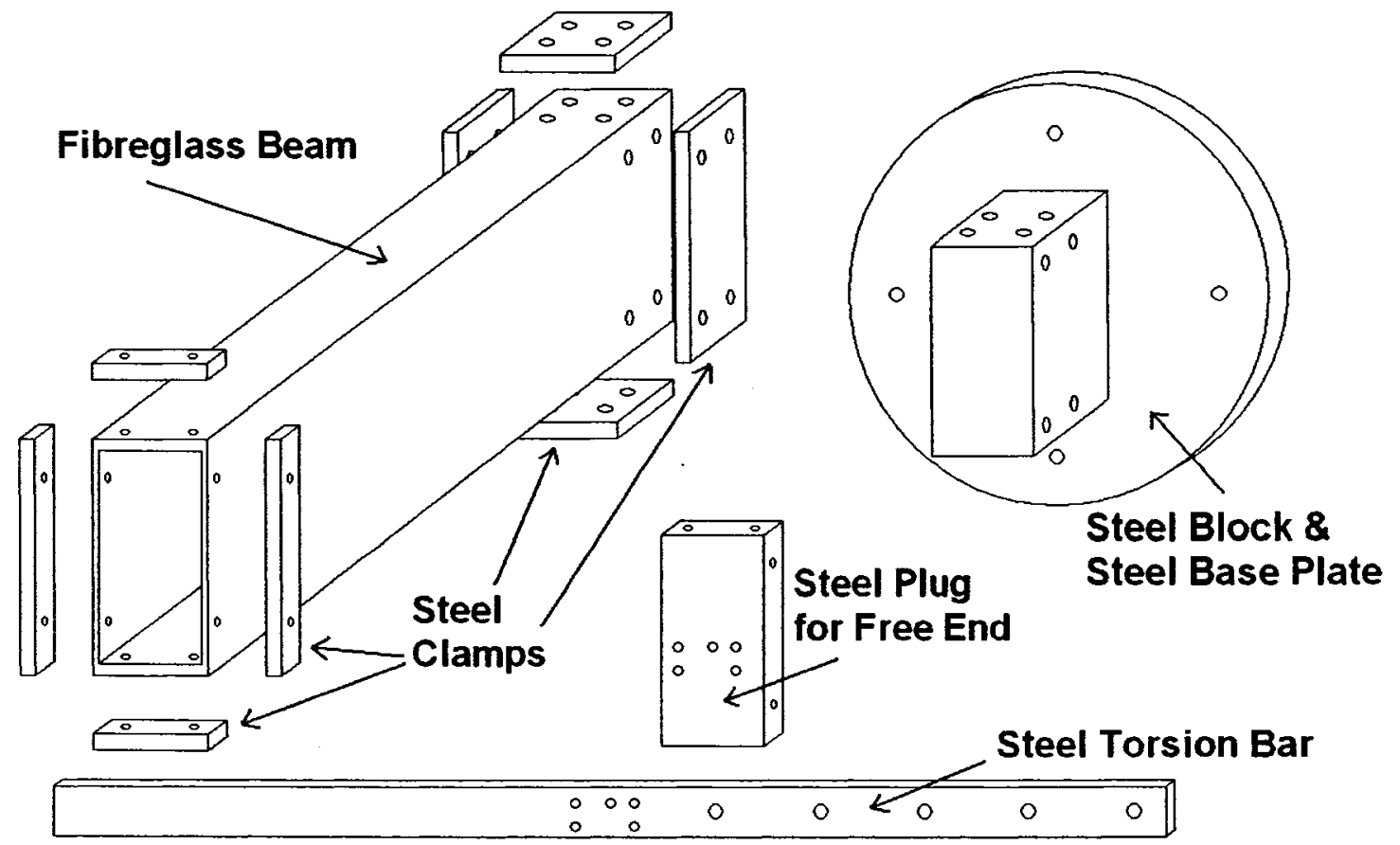

Figure 3.11 Fibreglass beam: fixtures and constraints

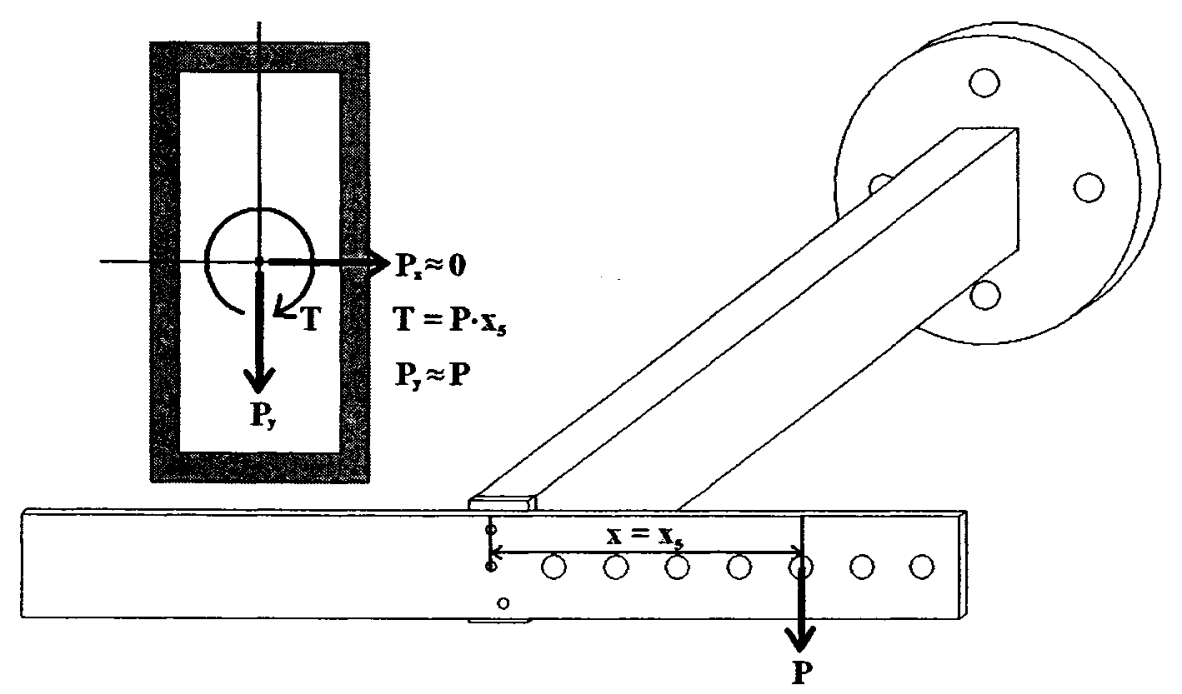

Figure 3.12 Resolving applied loads 


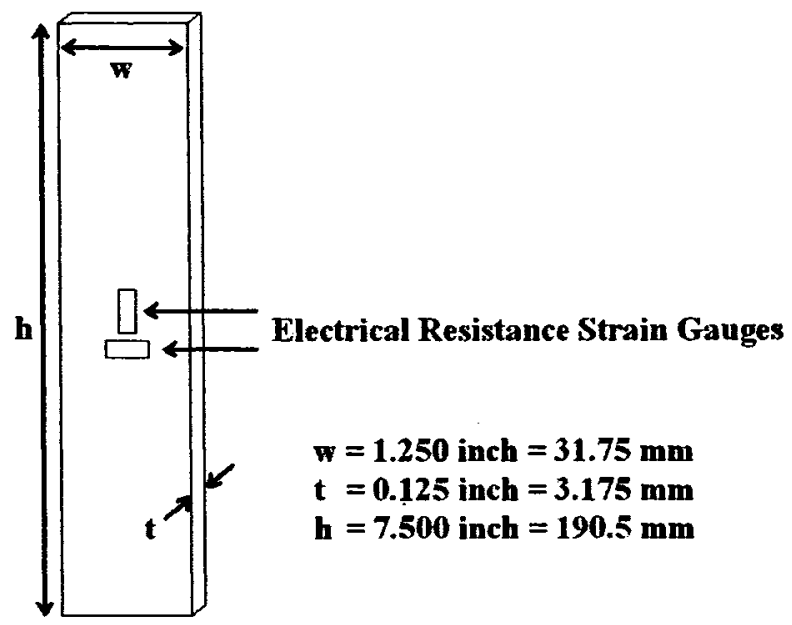

Figure 3.13 Material test coupon geometry of the fibreglass used

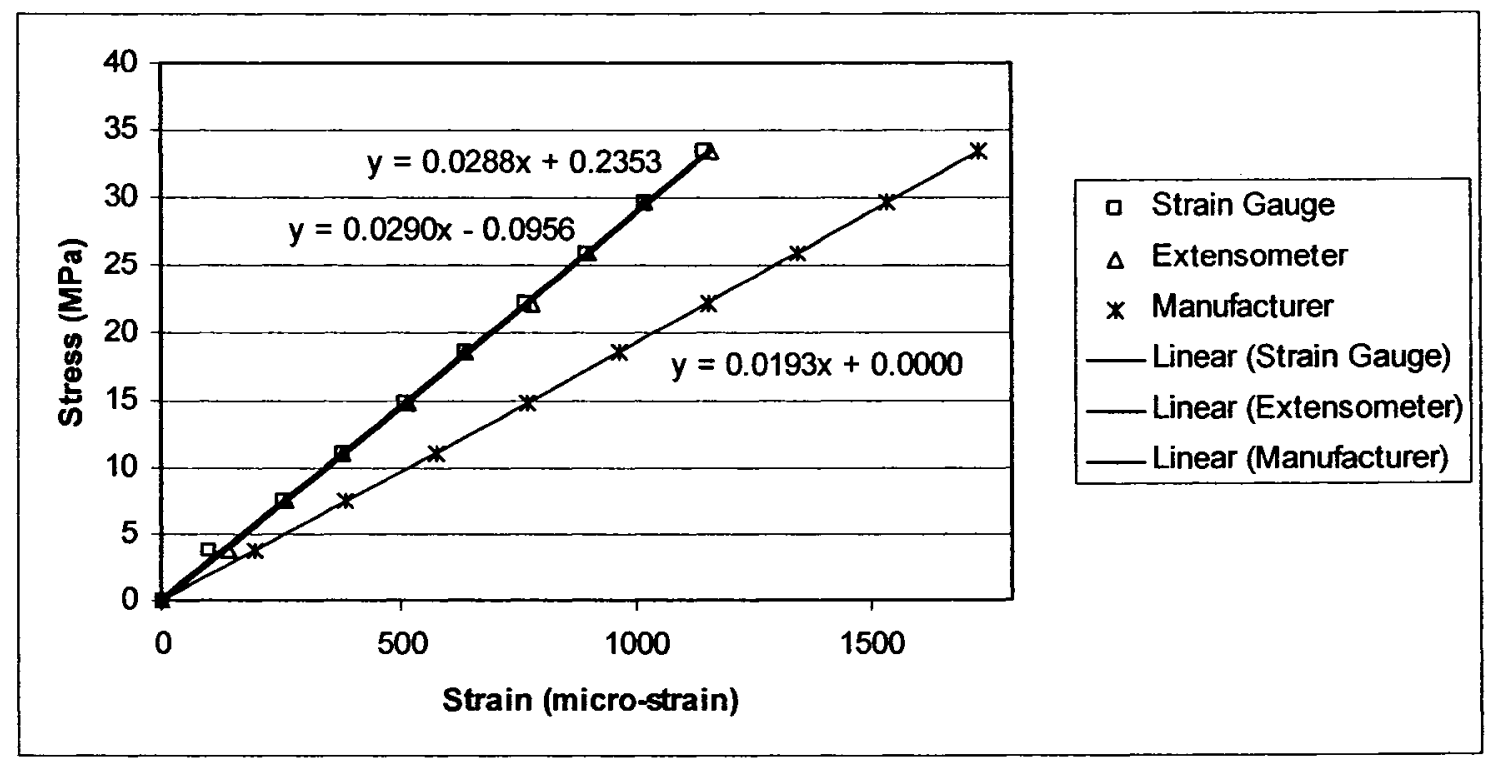

Figure 3.14 Axial stress versus axial strain plot for fibreglass test coupon 


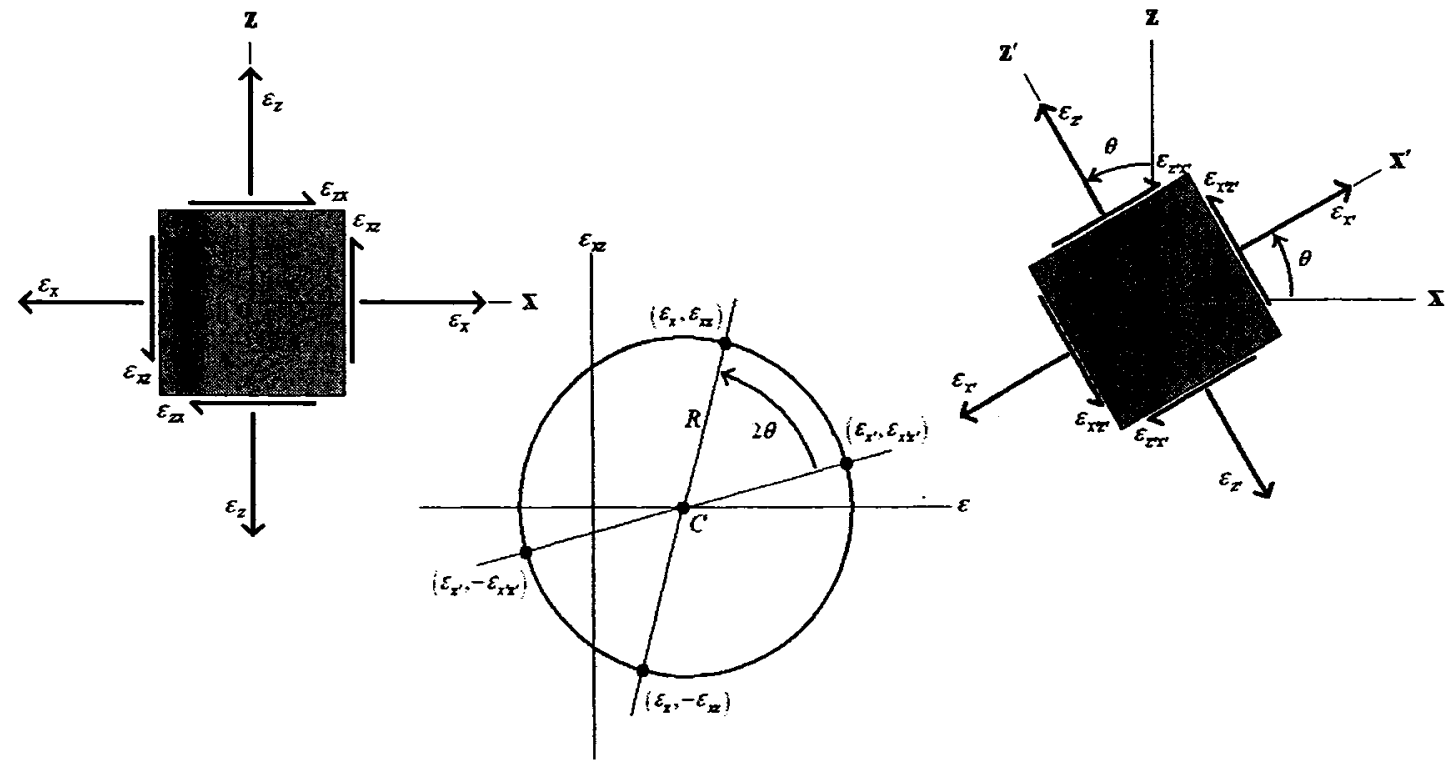

Figure 3.15 Strain coordinate transformation relationship and Mohr's circle

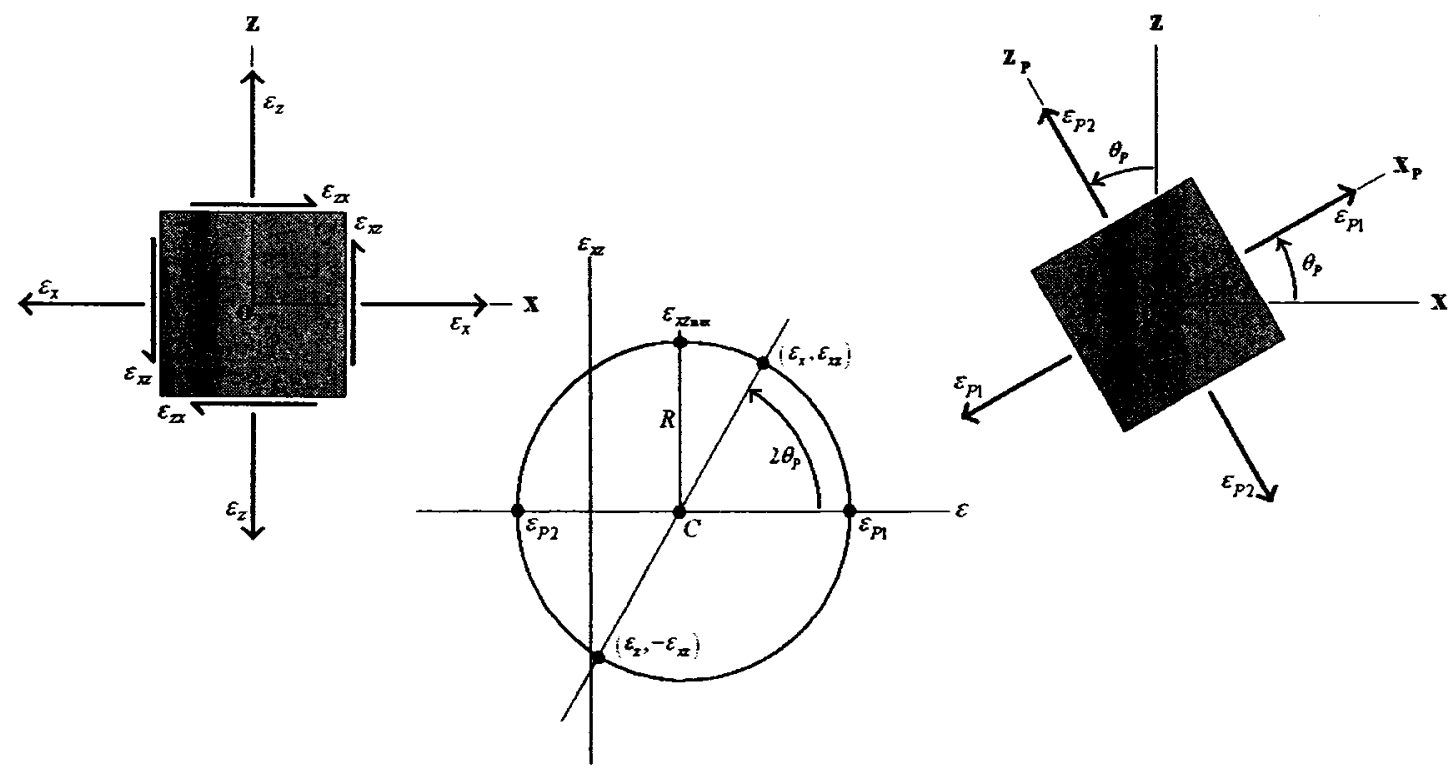

Figure 3.16 Principal strain orientation and Mohr's circle 


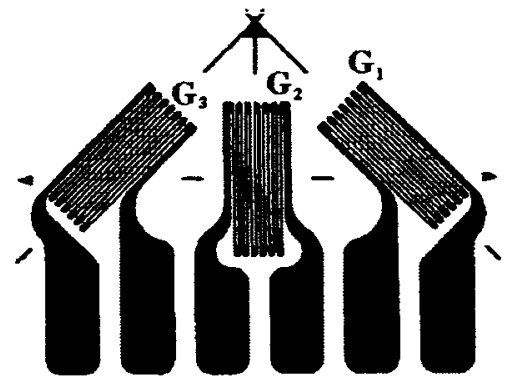

Rectangular Rosette $\begin{aligned} \theta_{12} & =45^{\circ} \\ \theta_{13} & =90^{\circ}\end{aligned}$

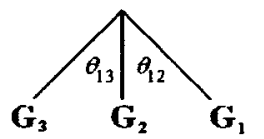

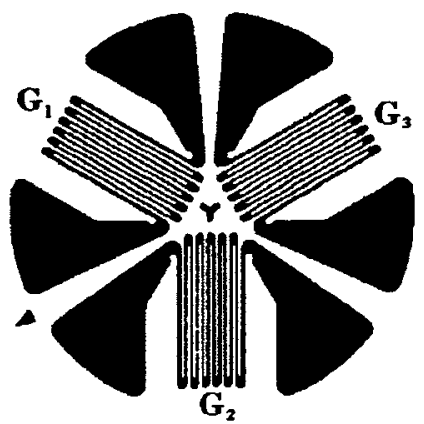

Delta Rosette

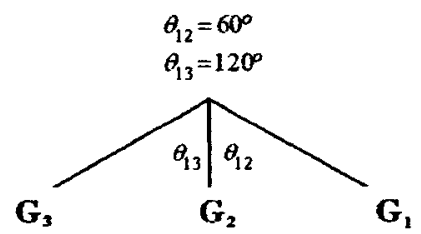

Figure 3.17 Typical electrical resistance strain gauge rosette configurations
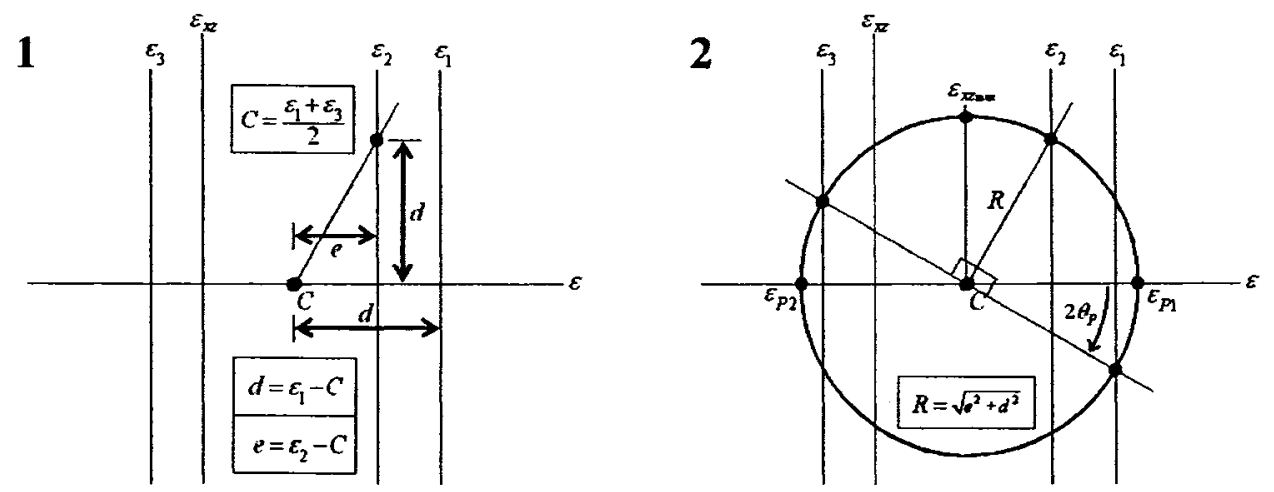

Figure 3.18 Construction of Mohr's circle using a rectangular strain gauge rosette 


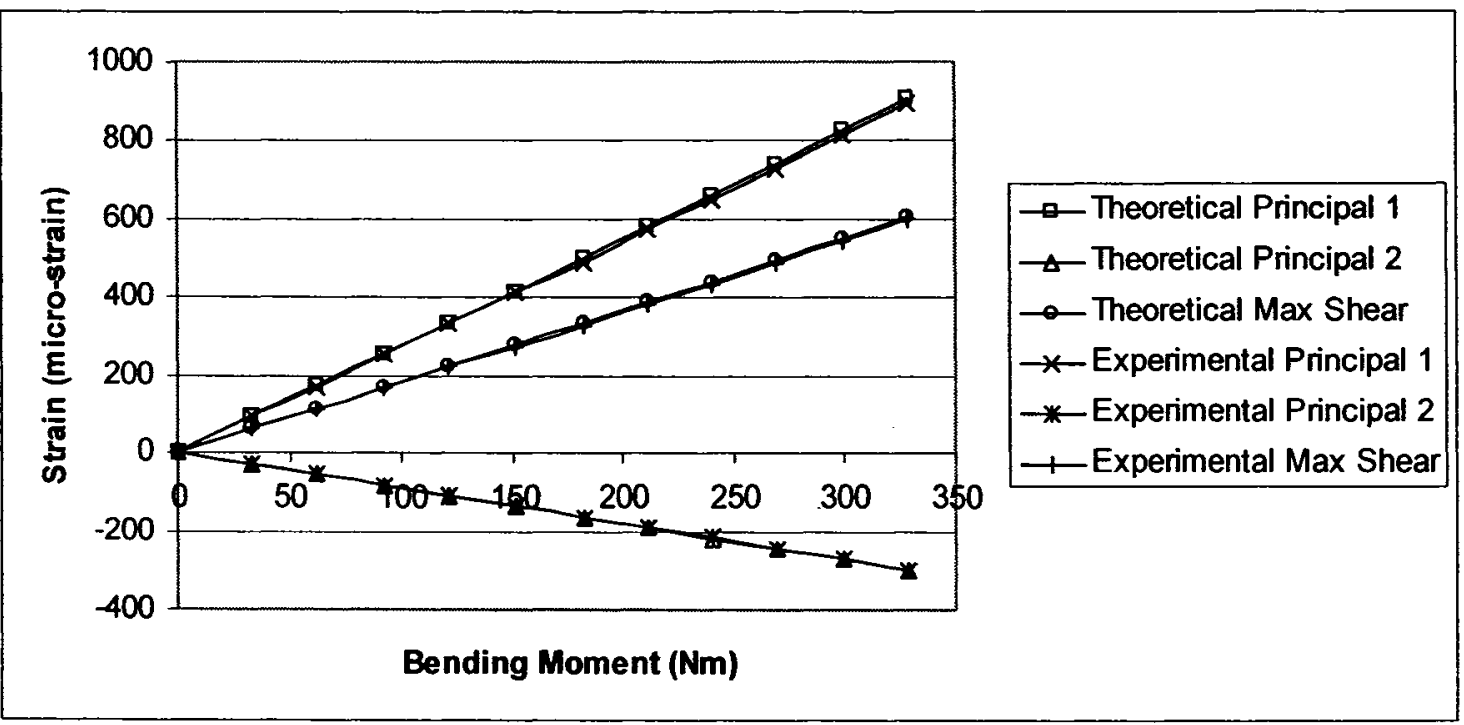

Figure 3.19 Bending strains measured by the top rosette for upright aluminum beam



Figure 3.20 Combined bending and torsion strains measured by the top rosette for upright aluminum beam 


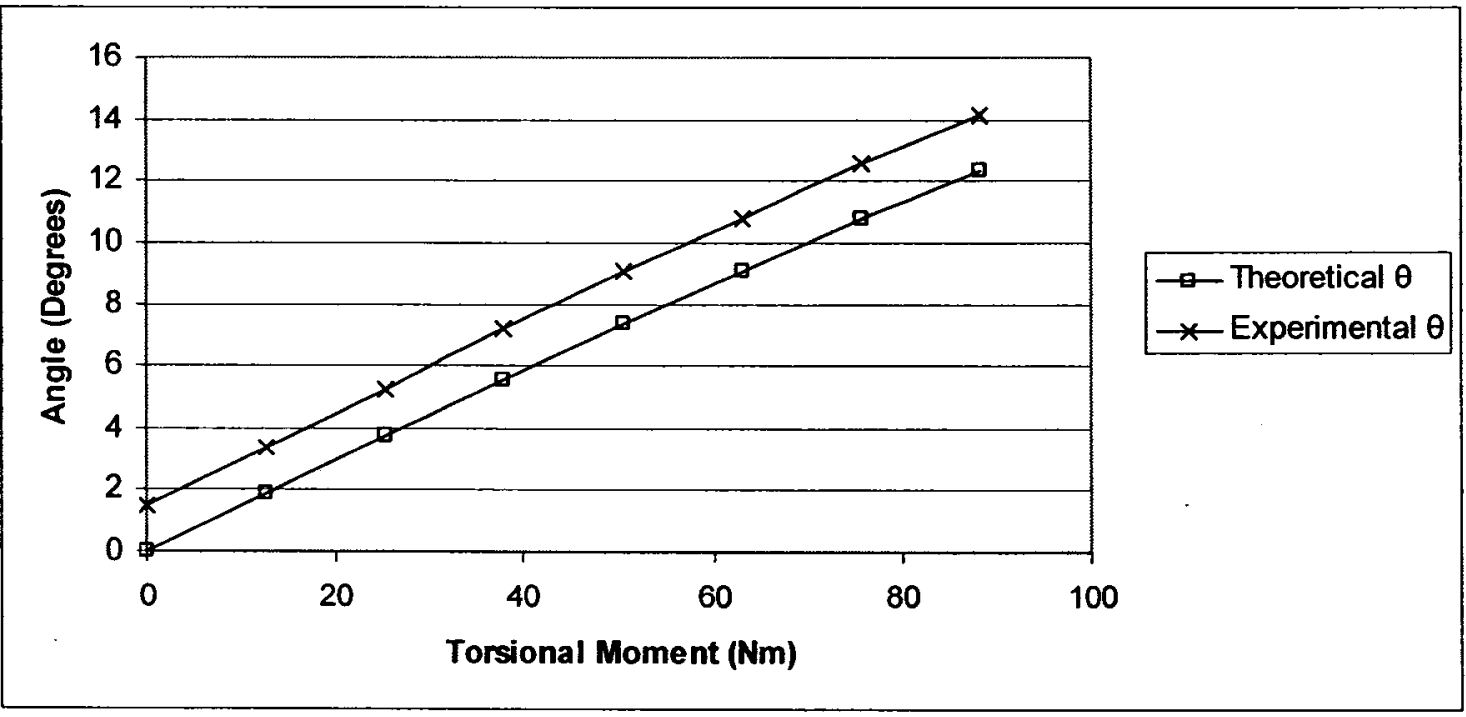

Figure 3.21 Principal angles measured by the top rosette for upright aluminum beam



Figure 3.22 Bending strains measured by the top rosette for inverted aluminum beam 




Figure 3.23 Combined bending and torsion strains measured by the top rosette for inverted aluminum beam

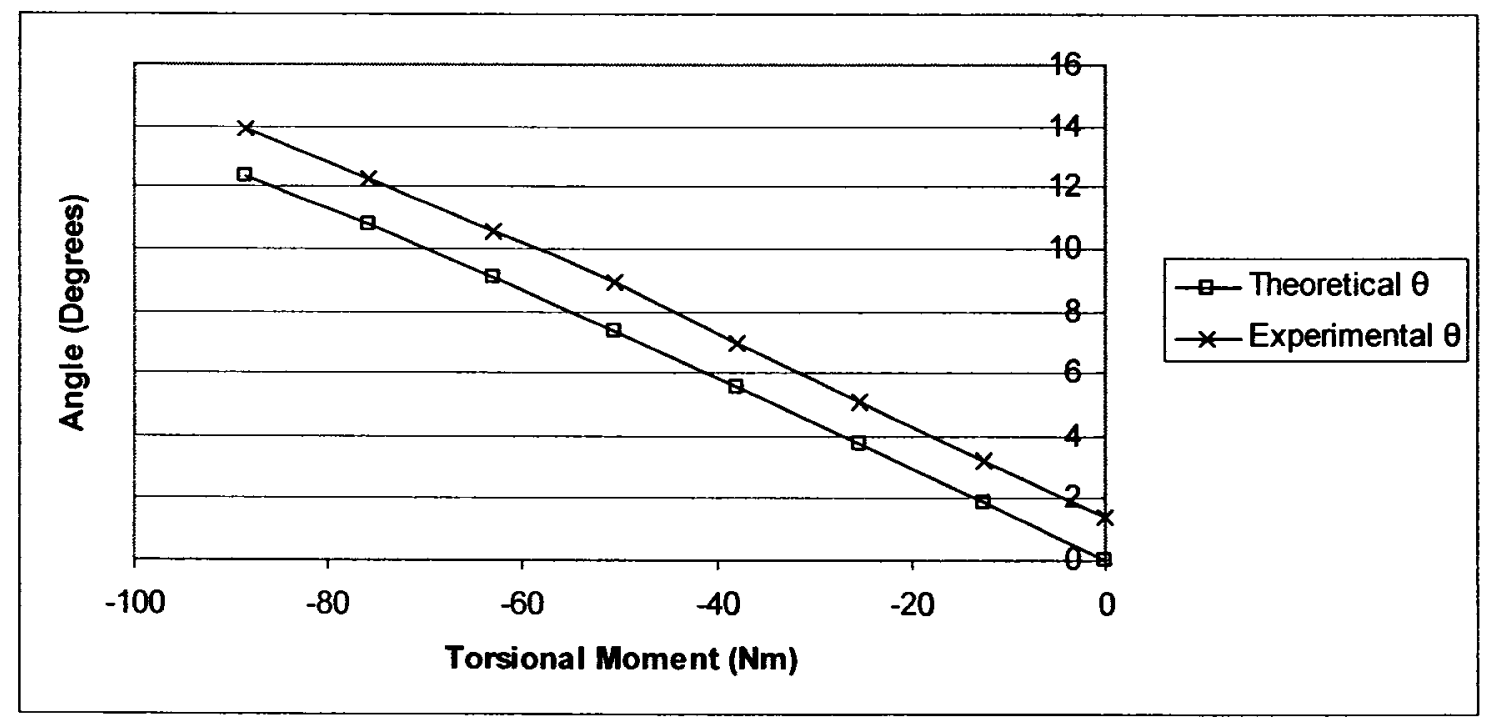

Figure 3.24 Principal angles measured by the top rosette for inverted aluminum beam 


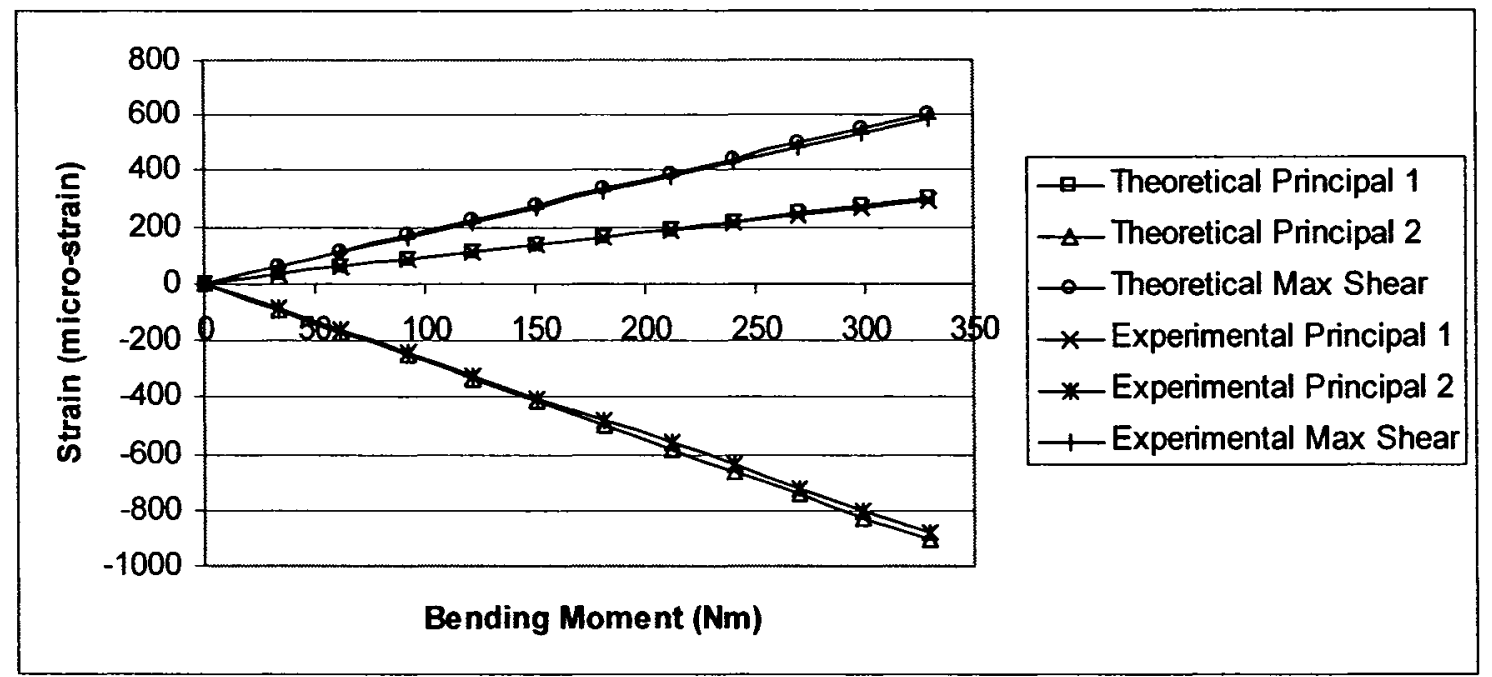

Figure 3.25 Bending strains measured by the bottom rosette for upright aluminum beam

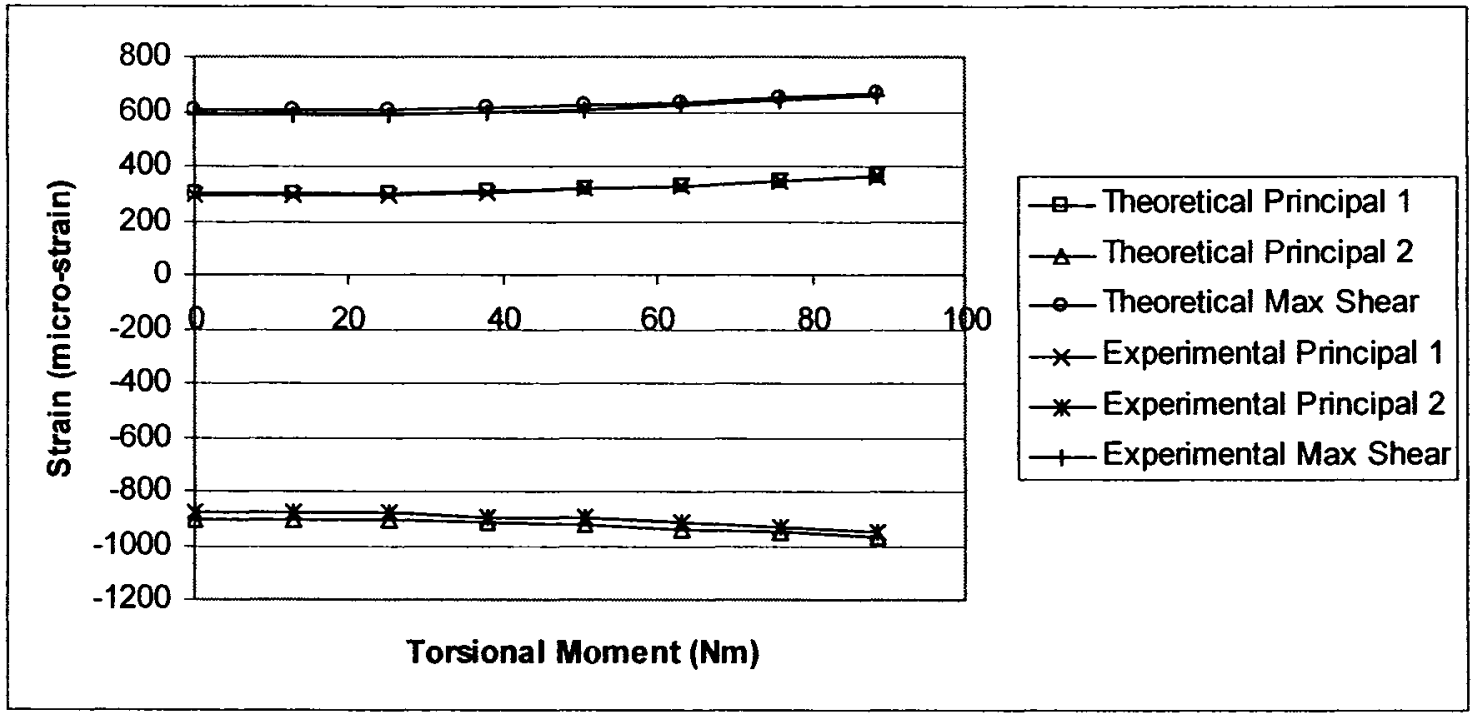

Figure 3.26 Combined bending and torsion strains measured by the bottom rosette for upright aluminum beam 


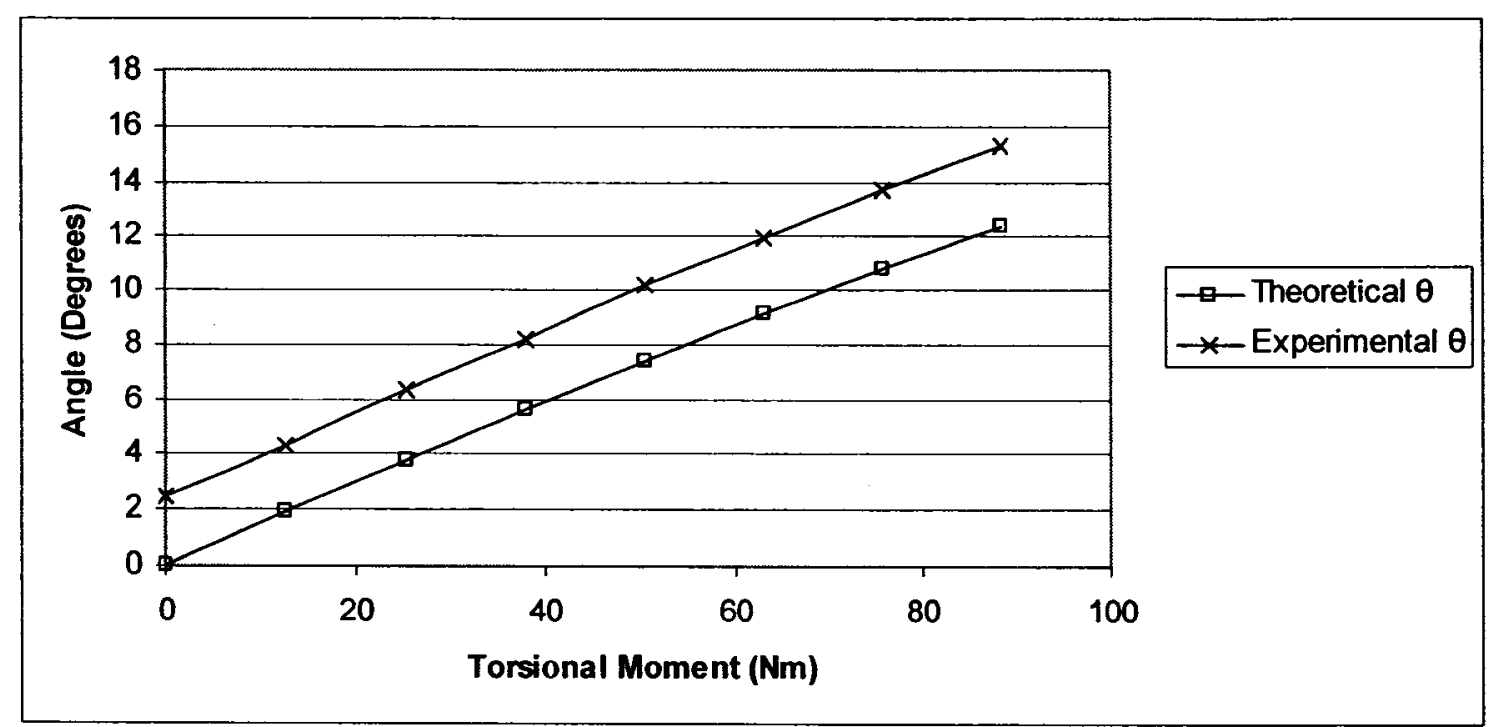

Figure 3.27 Principal angles measured by the bottom rosette for upright aluminum beam

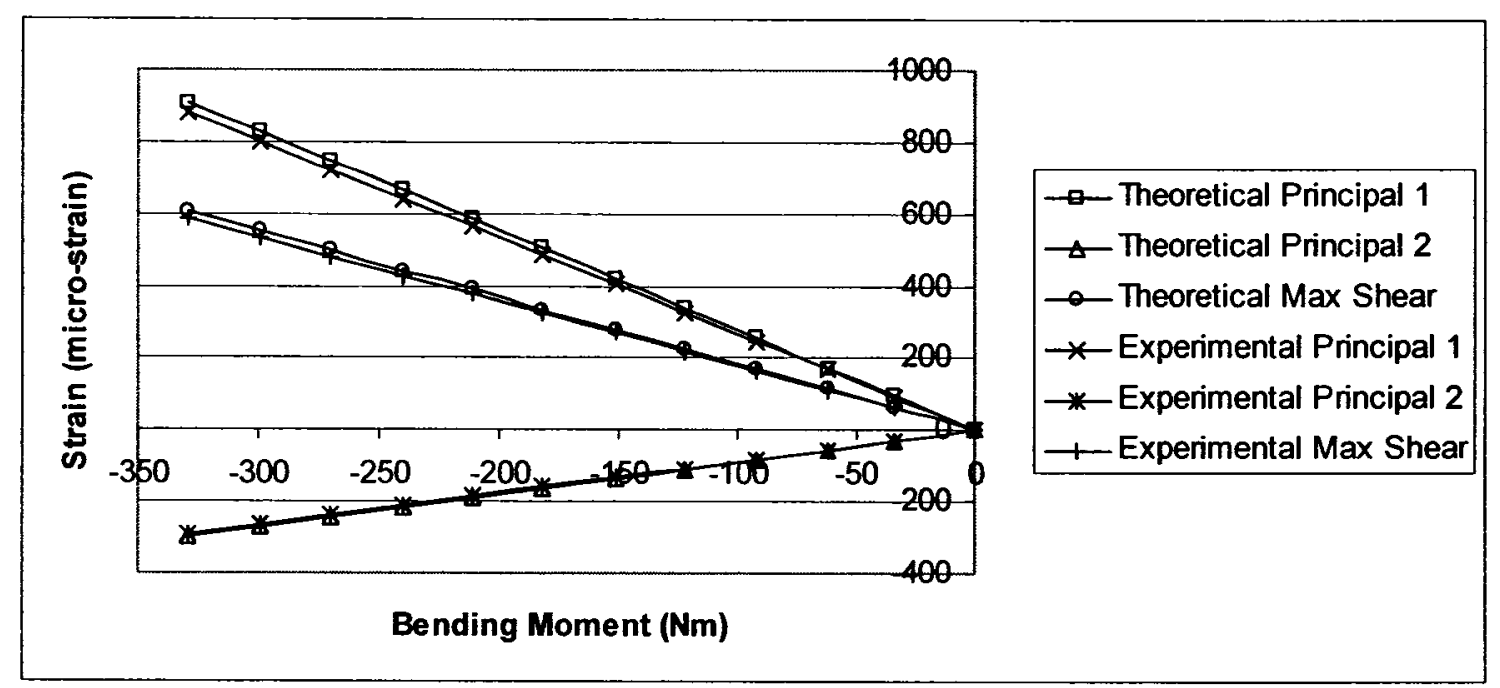

Figure 3.28 Bending strains measured by the bottom rosette for inverted aluminum beam 




Figure 3.29 Combined bending and torsion strains measured by the bottom rosette for inverted aluminum beam



Figure 3.30 Principal angles measured by the bottom rosette for inverted aluminum beam 


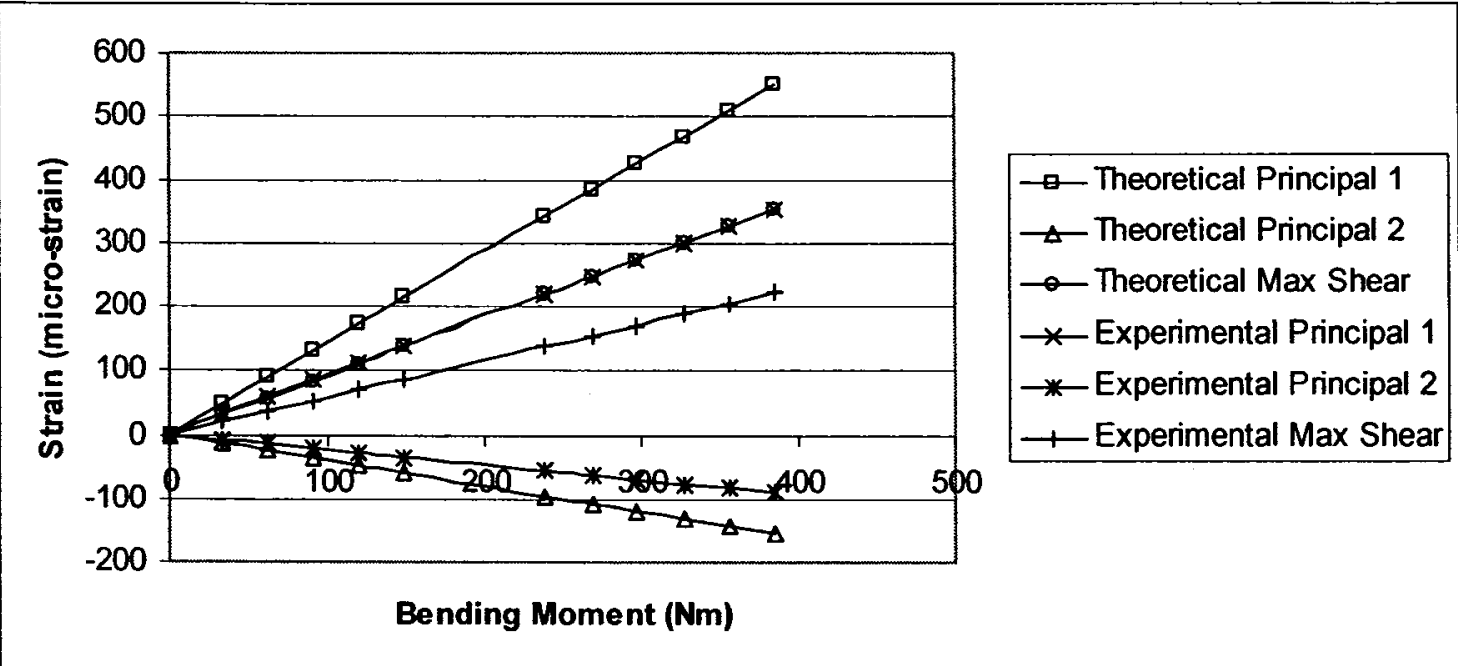

Figure 3.31 Bending strains in poor agreement for upright fibreglass beam

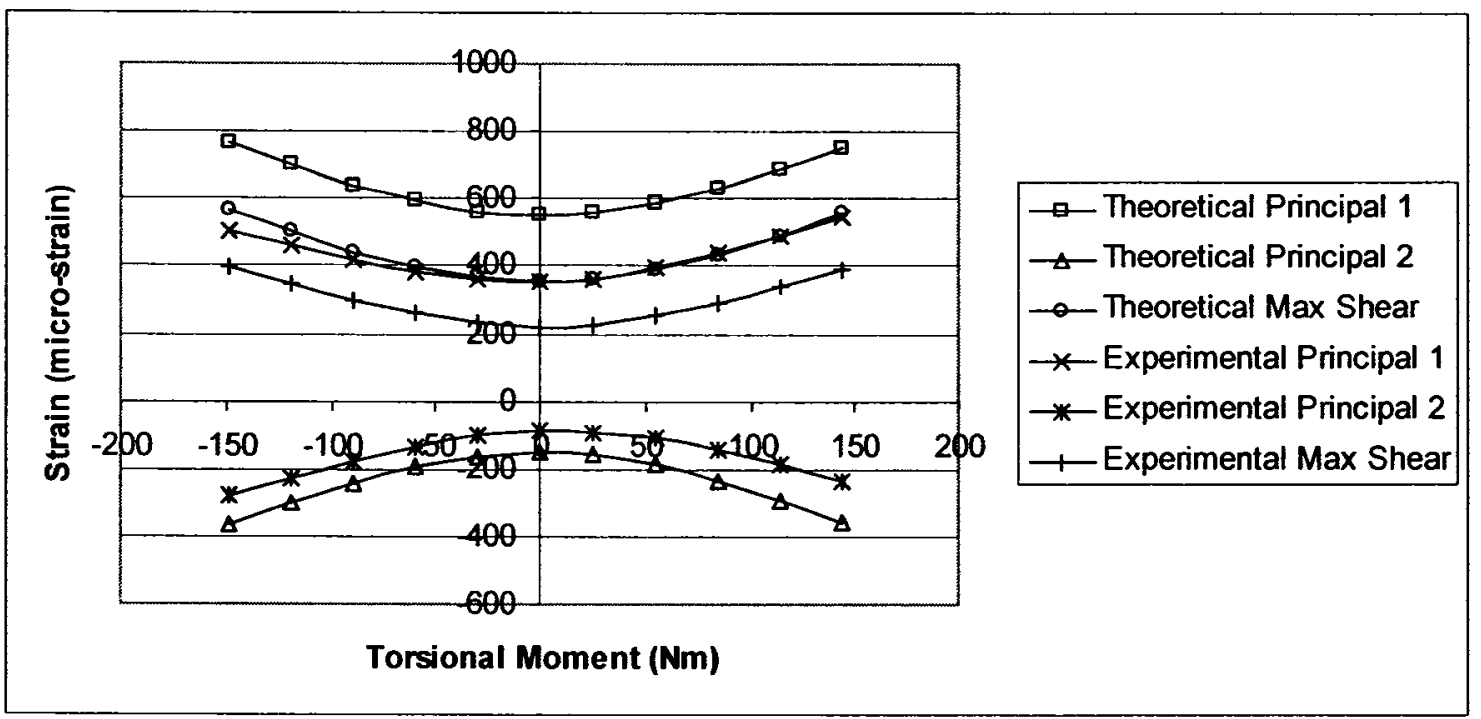

Figure 3.32 Combined bending and torsion strains in poor agreement for upright fibreglass beam 


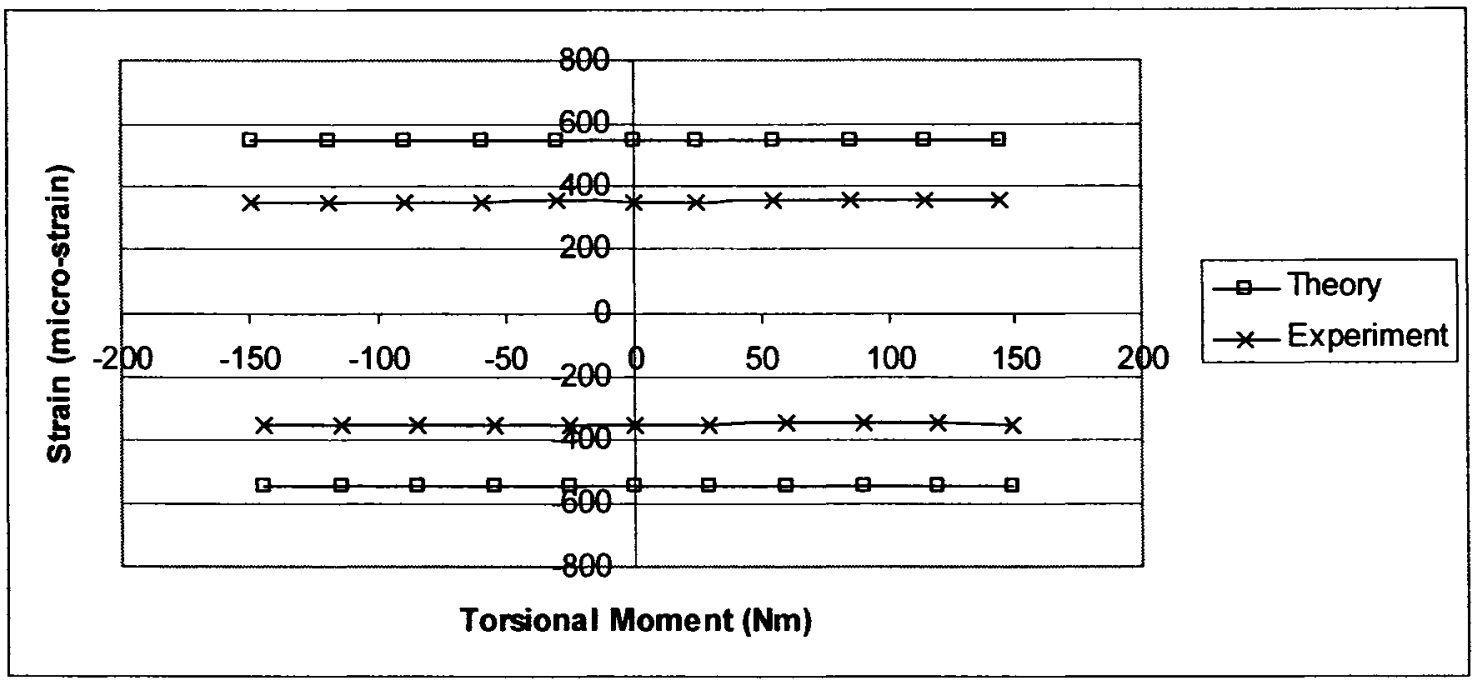

Figure $3.33 \alpha=0$ degree direction axial strains in poor agreement for fibreglass beam

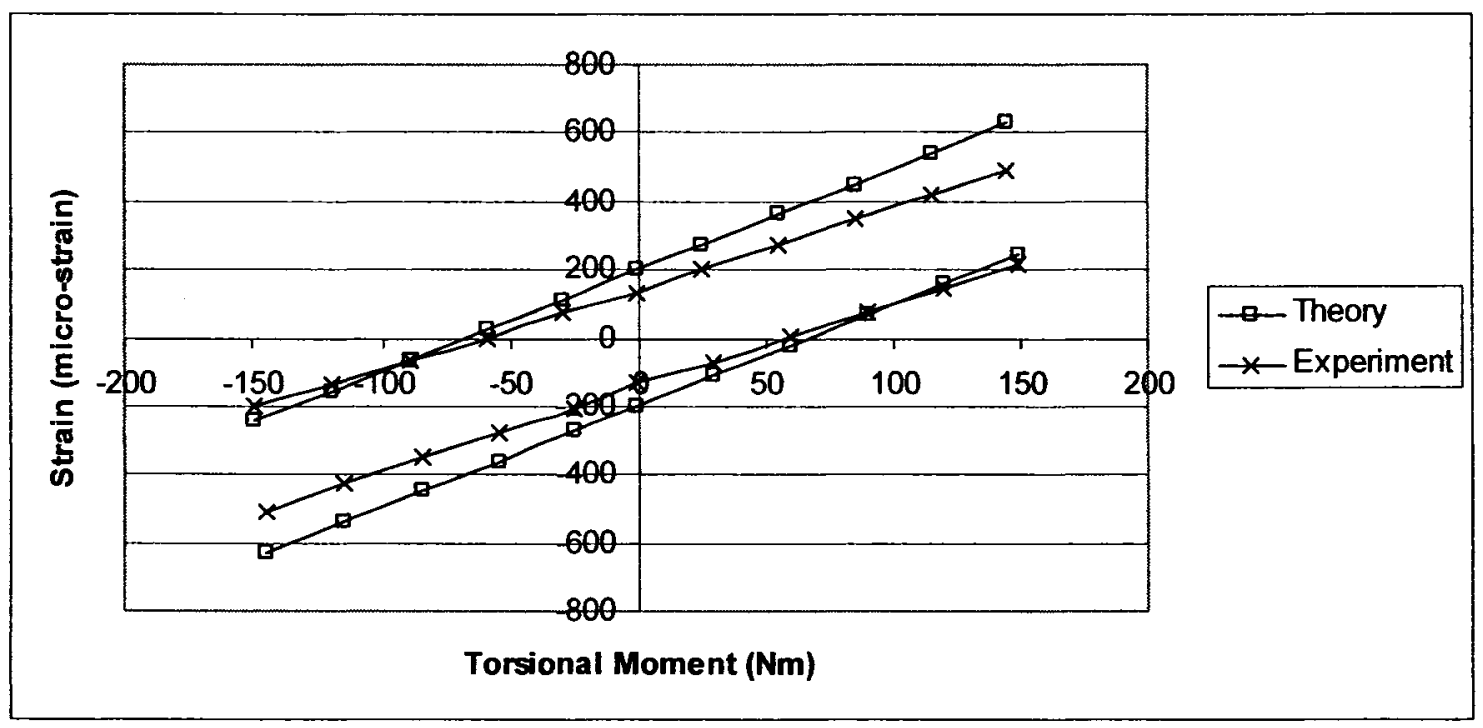

Figure $3.34 \alpha=45$ degree direction axial strains in poor agreement for fibreglass beam 


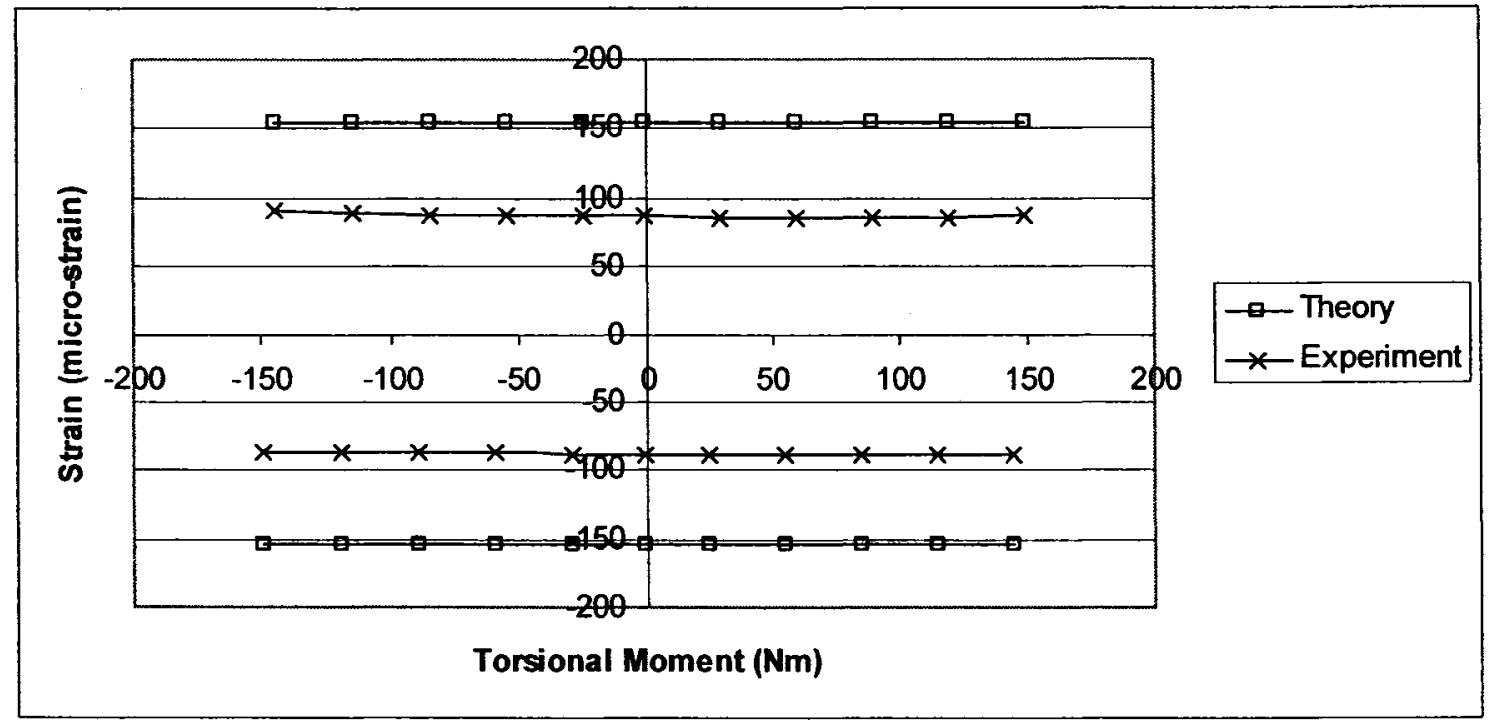

Figure $3.35 \alpha=90$ degree direction axial strains in poor agreement for fibreglass beam

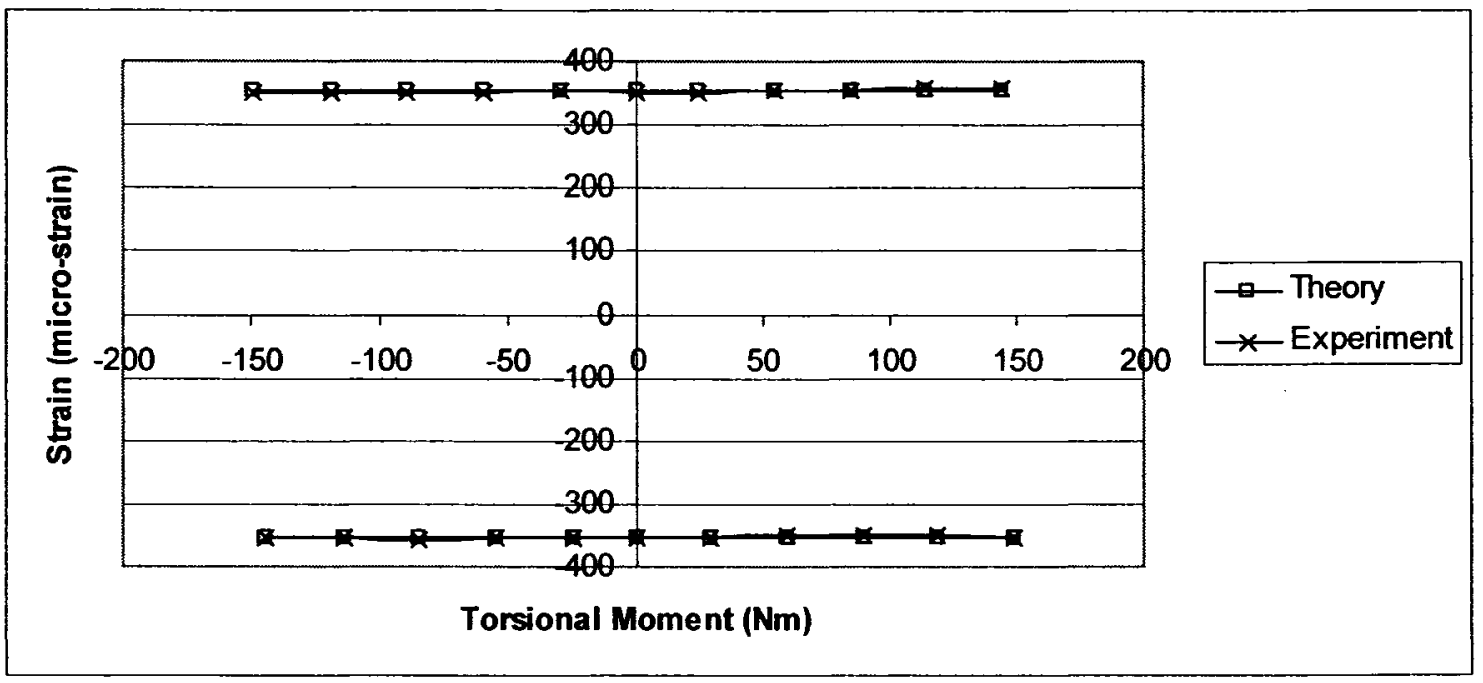

Figure $3.36 \alpha=0$ degree direction axial strains in good agreement for fibreglass beam 




Figure $3.37 \alpha=\mathbf{4 5}$ degree direction axial strains in good agreement for fibreglass beam

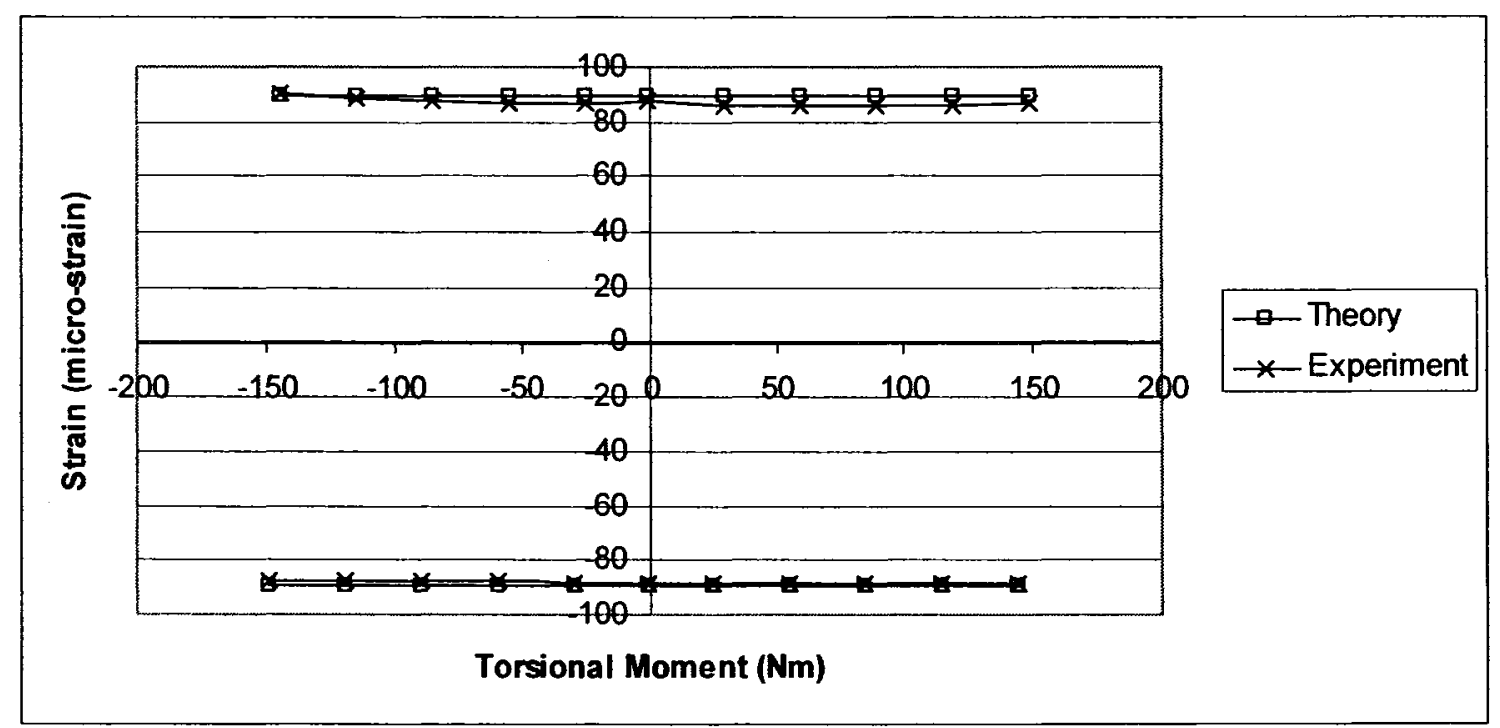

Figure $3.38 \alpha=90$ degree direction axial strains in good agreement for fibreglass beam 


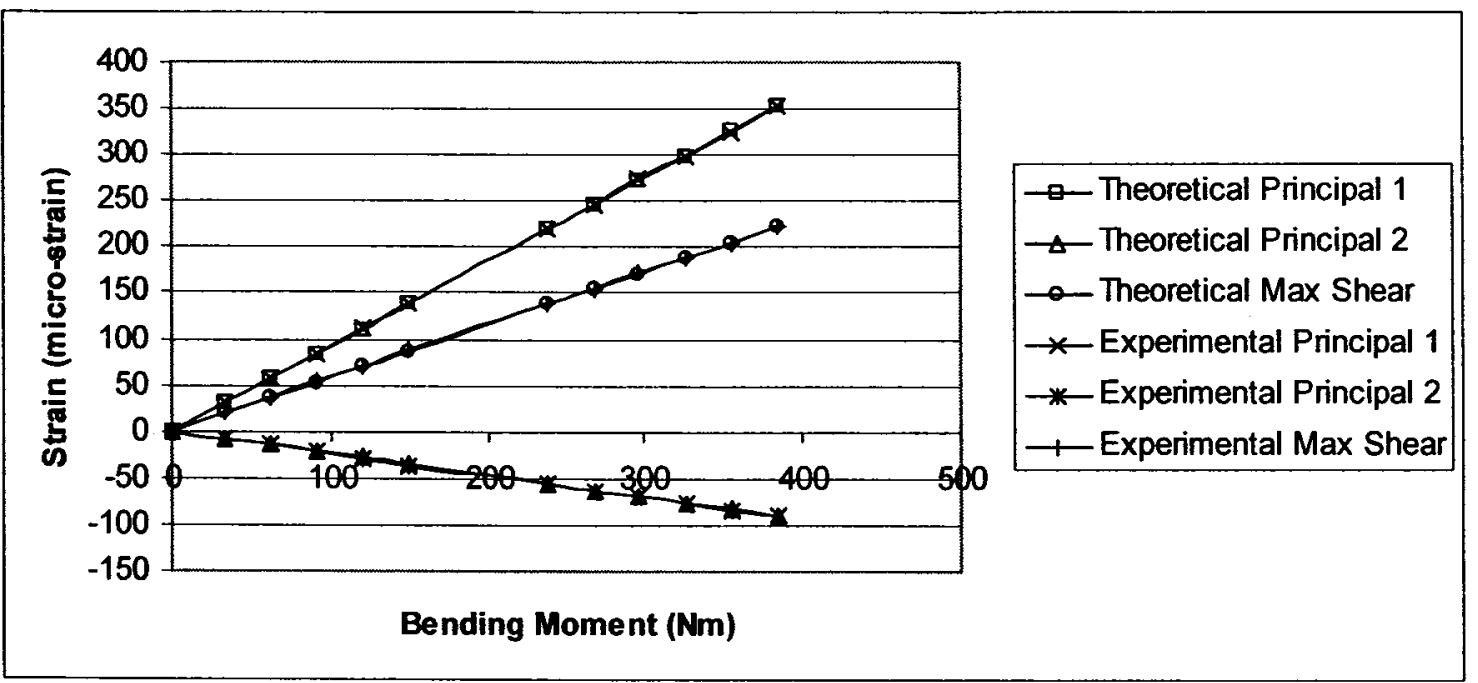

Figure 3.39 Bending strains in good agreement for upright fibreglass beam



Figure 3.40 Combined bending and torsion strains in good agreement for upright fibreglass beam 


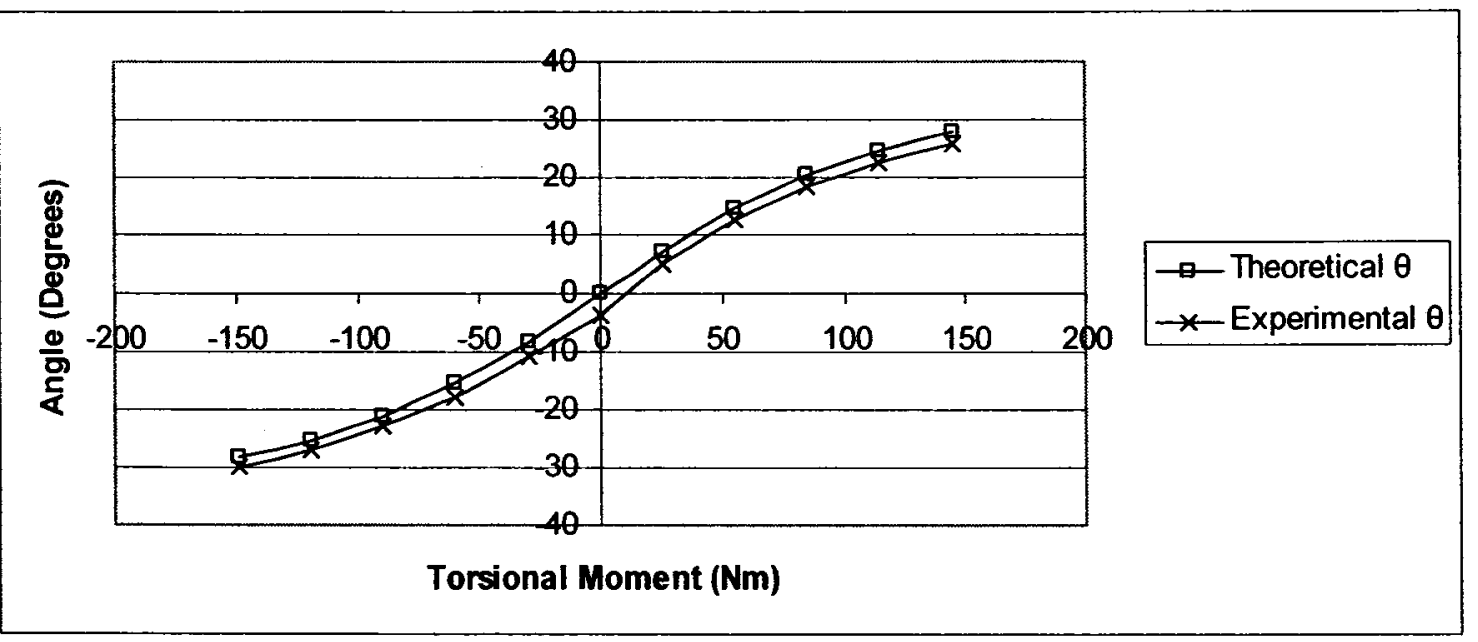

Figure 3.41 Principal angles in good agreement for upright fibreglass beam

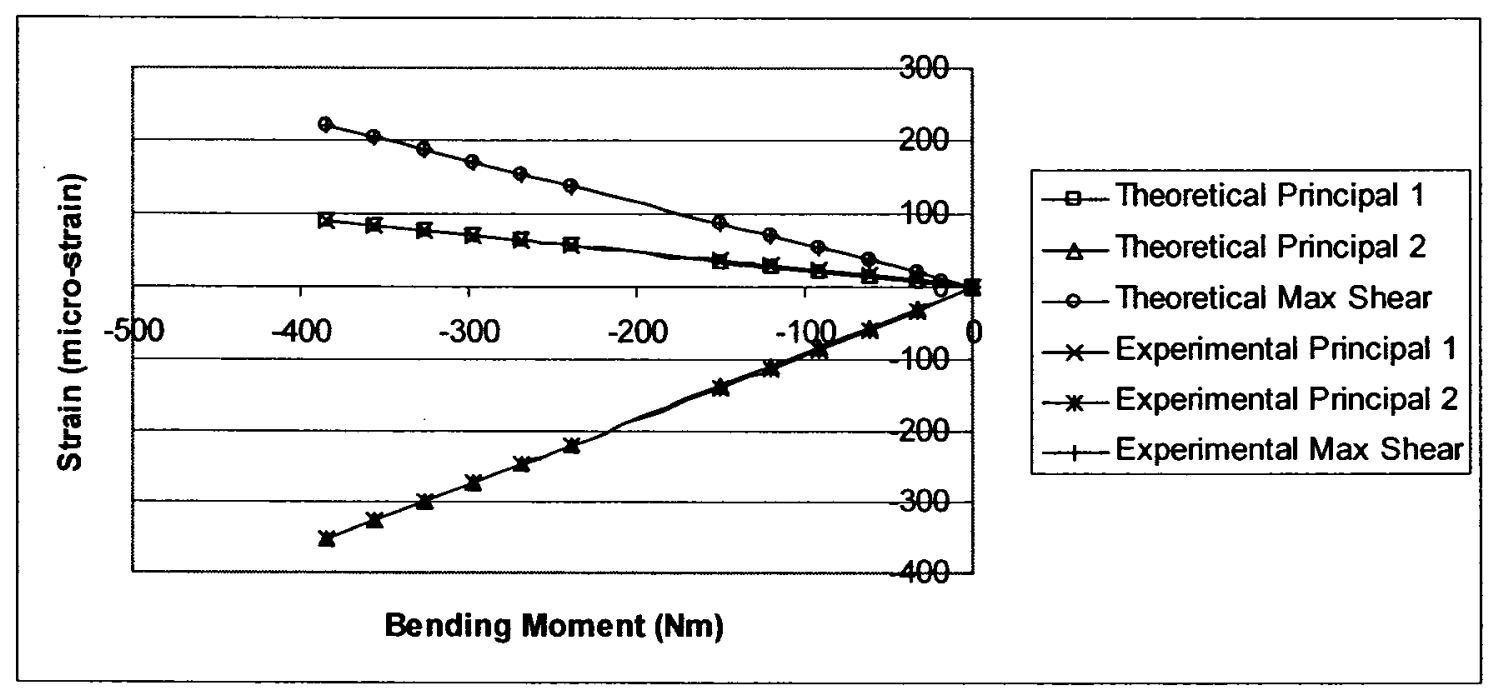

Figure 3.42 Bending strains in good agreement for inverted fibreglass beam 


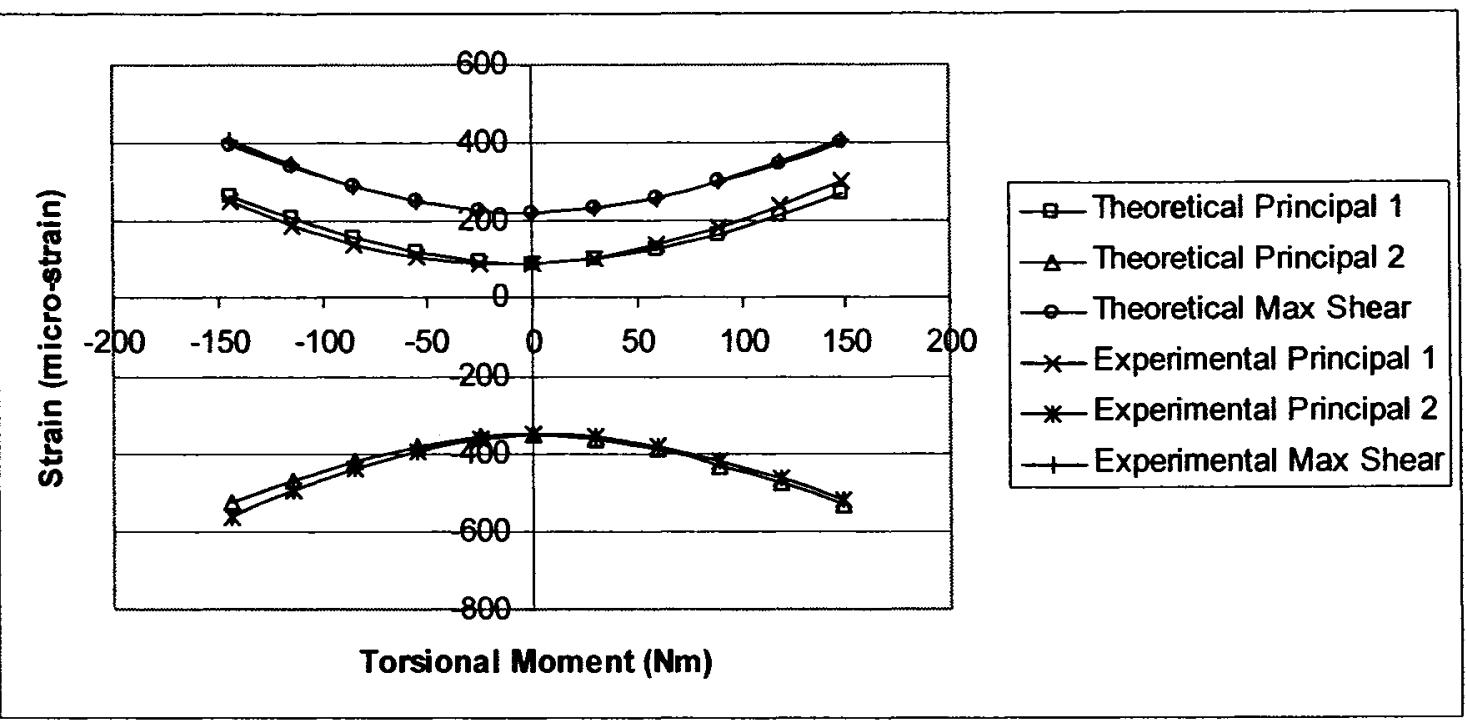

Figure 3.43 Combined bending and torsion strains in good agreement for inverted fibreglass beam

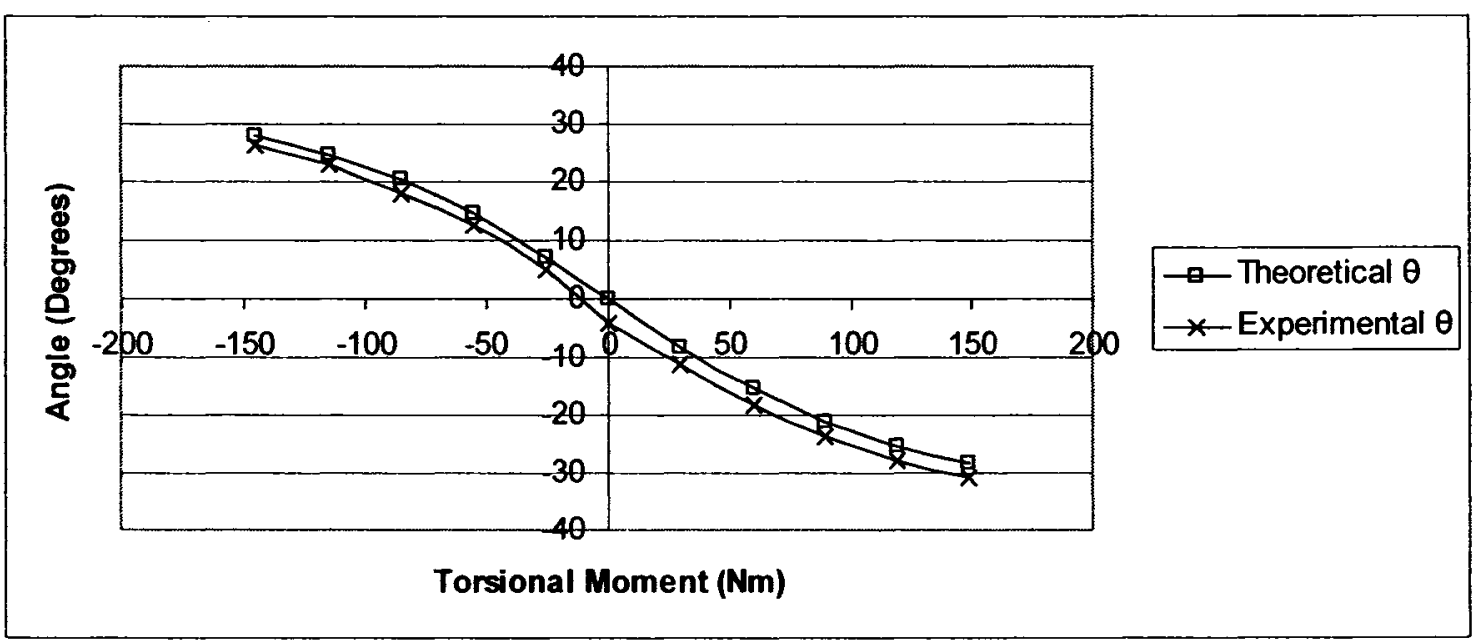

Figure 3.44 Principal angles in good agreement for inverted fibreglass beam 


\section{CHAPTER 4: FBG SENSOR TESTING AND RESULTS}

\subsection{Introduction}

The cantilever beam specimens validated in Chapter 3 were used to experimentally test FBG sensors. The experimental tests that were carried out using these sensors are described in this Chapter. A range of practical issues are addressed; they include sensor installation, data collection and processing, identifying potential sources of error and establishing quantitative estimates of their influence on the FBG strain measurement results. The FBG measured strains are then graphically and numerically compared with the theoretical and rosette measured axial strains for each sensor in its particular orientation. A discussion of the experimental results and errors is then presented, placing particular focus on the influence of transverse strain components in FBG sensor measurements and the interaction of FBG sensors with the adhesive. To this end, projections are made for the expected strain-optic behaviour of FBG sensors embedded within thin fibre reinforced laminate shell structures.

\subsection{Sensor Installation}

Each sensor was written into its own optical fibre using the phase mask technique as described in Section 2.3.1 and was installed on the test specimen surface using an 
adhesive. The cladding was stripped away from the fibre during the writing process, leaving the sensor bare. The adhesive will thus act as a cladding to the sensor, and must exhibit the appropriate optical and mechanical properties to prevent the loss of optical signal and provide adequate adhesion.

To prevent loss of optical signal, the refractive index of the adhesive must be lower than that of the fibre, however, refractive indices are not values typically published by adhesive manufacturers. Specialty coatings with known refractive indices could be used to re-coat the bare sensors prior to adhesion. However this would likely reduce the transfer of strain into the sensor as was suggested by Pak (1992). It was noted by Lin et al. (2005) that the adhesive layer between the sensor and specimen will behave similar to a coating and affect the transfer of strain into the sensor. No coatings were applied to sensors during these experiments.

The first adhesive used was a two part room temperature curing epoxy/hardener (LePage Regular Epoxy 11); epoxy is typical of the material used as the matrix in fibre reinforced composite laminate structures. The second adhesive used was a one part room temperature curing cyanoacrylate adhesive (Loctite Super Glue Brush-on) and is typical of the adhesive used for installation of strain gauges on test specimens. Neither manufacturer was able to provide mechanical properties for their adhesives, however values listed in Table 4.1 were estimated based on comparable adhesive products and the values measured by Olivier et al. (1992) using the nano-indendation technique for determining the modulus of thin coatings. 
The installation technique and procedure must be consistent for the mounting of each sensor. Geometric deviations such as adhesive thickness and curvature of the fibre may produce inconsistencies between sensor readings. Minimizing the thickness of adhesive between the sensor and specimen surface improves the transfer of strain to the sensor as confirmed by Lin et al. (2005). The bare sensors were therefore installed in direct contact with the specimen surfaces, and adhesive applied around them.

To prevent the sensor from taking a curved path while the adhesive cured, a slight tension was applied to the fibre using pieces of adhesive tape. The epoxy/hardener took several hours to fully cure at room temperature, during which the tape slowly released tension on the fibre, and resulted in an arc-shaped path. Additional constraints were therefore applied as shown in figure 4.1 by clamping a rubber pad with a channel cut into it over the sensor to hold it in alignment while the adhesive cured; a release film was used to prevent the rubber pad from adhering to the specimen. The cured epoxy/hardener adhesive surrounding the sensor was minimal, coating only the upper and side surfaces and filleting the lower section of the sensor to the test specimen as shown in figure 4.1. The cyanoacrylate adhesive cured within minutes and required no additional constraints to keep the sensors in alignment. The cured cyanoacrylate adhesive surrounding the sensor formed a slightly convex shape as shown in figure 4.1.

Tensions and contact pressures applied during curing of the adhesive leave residual strains in the sensor, which cause a permanent distortion of the Bragg peak in its optical signal. Sensors embedded in a composite structure would be exposed to vacuum or autoclave pressures and temperatures while curing and may result in significant signal 
distortion. The Bragg peaks of sensors may also reduce in strength during the cure cycle of a laminate if using a high temperature adhesive. This is because exposure to elevated temperatures increases diffusion rates, and would thereby cause the Bragg grating pattern to fade because its original formation was due to a diffusion based process. A new generation of Bragg gratings, known as surface relief Bragg gratings, are under investigation by Smith et al. (2006) where the grating is physically etched into the core of the fibre, making the sensors stable when exposed to high temperatures. As long as the distorted and faded optical signal contains a noticeable and measurable Bragg peak, residual strain and temperature exposure effects during the installation process can be neglected. This is because additional applied strain will be superimposed over the residual strain, thereby shifting the distorted optical signal according to the same strainoptic relationship as an undisturbed sensor.

Each sensor was connected to the optical circuit during installation to examine distortion and changes of the optical signal. Figure 4.2 shows the optical signal of a tilted FBG sensor measured in transmission before and after installation. The noticeable changes are a wavelength shift of all the peaks caused by the applied tension, and changes in wavelengths and strengths of the other peaks, which correspond to a change in the power coupling behaviour of the tilted gratings to radiation modes; the changes seen in these peaks are due to the refractive index change from air to adhesive surrounding the sensor core. 


\subsection{Sensor Properties and Optical Signals}

Eight FBG sensors were experimentally tested in total; two sensors on the aluminum beam and six sensors on the fibreglass beam. Each sensor was adhesively bonded onto either the top or bottom surface of the specimen at approximate orientations of 0,45 , or 90 degrees. The specific location and orientation of each sensor was measured after installation and is listed in Table 4.2 along with the type of adhesive used.

The eight sensors were not identical in design, resulting in a range of optical signals, but all contained a noticeable and measurable Bragg peak. Some sensors had dual overwritten gratings, some had tilted gratings, and some were typical planar gratings. They should, in theory, all reflect a Bragg peak corresponding to their grating pitch along the centreline of the fibre, and should all respond equally to applied strains. Some sensors were measured using the transmission spectrum, while others were measured using the reflection spectrum or both. The final optical signals, after distortions caused during installation, are shown in figures $4.3-4.10$ for sensors 1 - 8 respectively.

The mechanical and optical properties of an FBG sensor are essentially the same as the optical fibre they are written in. All experimentally tested sensors were written in germanium-doped silica glass optical fibre which, according to Prabhugoud and Peters (2006), has the mechanical and optical properties as listed in Table 4.1. The diameter of the multi-mode fibre was measured using micrometers to be approximately $125 \mu \mathrm{m}$. 


\subsection{Collection and Processing of Optical Signal Data}

Due to the absolute resolution of the SWS-OMNI system, the wavelength accuracy of any given data set is inherently $\pm 2 p m$, equating to a high strain resolution of $\pm 1.6 \mu \varepsilon$ for a $1550 \mathrm{~nm}$ FBG. Table 4.3 shows a typical set of the raw optical signal data that was collected for each load and there exists a Bragg peak. The wavelength at which the minimum insertion loss value occurs was not consistently located within the peak. The tip of the peak flattens as shown in figure 4.11 , allowing the minimum to be located randomly among several adjacent points. This random signal error can be reduced by applying a simple computational algorithm to each data set and consistently determine a centre wavelength for the Bragg peak as described below.

The peak is generally located by finding the minimum insertion loss of the signal and applying any offset may be required if the minimum value is not located in the Bragg peak as is typical of a tilted grating shown in figure 4.3. The wavelengths where the optical signal intersects a designated power threshold on each side of the peak such as those shown in figure 4.12 are calculated by linearly interpolating within the corresponding measurement interval. The centre wavelength of the peak is computed as given by equation 4.1, where $\lambda_{R}$ and $\lambda_{L}$ are the wavelengths at threshold, Carson (2006).

$$
C W=\frac{\left(\lambda_{R}+\lambda_{L}\right)}{2}
$$

The threshold is typically set at $3 d B$ above the minimum insertion loss, but can be set to any value intersecting the optical signal peak where it is consistently clear. The centre wavelength for each sensor at no load is listed in Table 4.2 and shown graphically in the 
optical signal plots of figures 4.3 to 4.10 . The improvement to signal alignment using this method is shown by figure 4.12 and corresponds to a reduction in random error by approximately $\pm 12 \mathrm{pm}$ for transmission and $\pm 20 \mathrm{pm}$ for reflection signals, or $\pm 10 \mu \varepsilon$ and $\pm 16 \mu \varepsilon$ respectively for a $1550 \mathrm{~nm}$ FBG sensor.

The shift of centre wavelength shown in figure 4.13 is calculated for each applied load by referencing the centre wavelength of a zero load data set. Due to thermal sensitivity of FBG sensors, the centre wavelength may vary slightly over time. Zero load data sets were therefore recorded immediately prior to any series of sensor measurements to be used as the corresponding centre wavelength reference. An additional zero load set was recorded at the end of each series to observe any change in ambient temperature that may have occurred. The 'false' strain reading indicated by the zero load set at the end of a measurement series would equate to a change in ambient temperature as shown in figure 4.14. This figure equates thermal and strain induced centre wavelength shifts of a $1550 \mathrm{~nm}$ sensor and accounts for thermal expansion of the test specimen using the appropriate coefficients of thermal expansion as listed in Table 3.3. This method does not eliminate the effects of localized random temperature fluctuations, which would introduce random errors into the observed strain measurements proportional to their magnitudes as shown by figure 4.14. A temperature discrimination technique was not deemed necessary during these experiments because of the relatively stable thermal environment in the CLLIPS laboratory. 


\subsection{Sensor Experimental Results}

Having calculated the shift of centre wavelength for each applied load, it can be converted into an apparent strain using equation 2.7 with an optical gauge factor of 0.795 , as derived in Chapter 2. The apparent strain of each FBG sensor is compared to the theoretical and rosette measured strain components of the test specimen that are acting in the axial orientation of the sensor. This comparison is presented graphically for each sensor by two figures; the first figure compares strain values for applied bending loads, while the second figure compares strain values for application of combined bending and torsion at constant bending load values; figures $4.15-4.30$ thus correspond to FBG 1 - 8 sequentially in pairs. Tables 4.4 and 4.5 present a numerical comparison of these strain values at maximum and minimum applied load combinations.

\subsubsection{Aluminum Specimen}

FBG 1 and FBG 2 were installed on the aluminum test specimen using epoxy/hardener adhesive at the locations indicated in Table 4.2. Both sensors were of the tilted grating type. Their optical signals, as shown in figures 4.3 and 4.4 , were measured in transmission.

The apparent strain measured by FBG 1 is in agreement within $2 \%$ and a difference of 13 micro-strain of the theoretical axial strain component as shown in Table 4.4. The range of axial strain applied to this sensor was approximately $-900 \mu \varepsilon$ to $+900 \mu \varepsilon$, corresponding to an approximate Bragg wavelength shift of $-1.11 \mathrm{~nm}$ to $+1.11 \mathrm{~nm}$. The measured response of FBG 1 to applied bending loads was linear as shown in figure 4.15, 
and because it was oriented at approximately 1.75 degrees, its measured response to applied torsional loads was negligible as shown in figure 4.16 .

The apparent strain measured by FBG 2 is in agreement within $4 \%$ and a difference of 13 micro-strain of the theoretical axial strain component as shown in Table 4.4. The range of axial strain applied to this sensor was approximately $-630 \mu \varepsilon$ to $+630 \mu \varepsilon$, corresponding to an approximate Bragg wavelength shift of $-0.775 \mathrm{~nm}$ to $+0.775 \mathrm{~nm}$. The measured response of FBG 2 to applied bending loads was linear as shown in figure 4.17 , and because it was oriented at approximately -42 degrees, its measured response to applied torsional loads was also linear as shown in figure 4.18 .

\subsubsection{Fibreglass Specimen}

FBG 3, FBG 4, and FBG 5 were installed on the fibreglass test specimen using epoxy/hardener adhesive at the locations indicated in Table 4.2. FBG 3 had dual Bragg peaks in its optical signal and was measured in reflection as shown in figure 4.5 , whereas FBG 4 and FBG 5 had only single Bragg peaks in their optical signals and were measured in transmission as shown in figures 4.6 and 4.7 respectively. FBG 6, FBG 7, and FBG 8 were installed on the fibreglass test specimen using cyanoacrylate adhesive at the locations described in Table 4.2. All three sensors had single Bragg peaks and were measured in both transmission and reflection as shown in figures $4.8-4.10$.

The apparent strain measured by FBG 3 is in agreement within $6 \%$ and a difference of 6 micro-strain of the theoretical axial strain component as shown in Table 4.5. The range of axial strain applied to this sensor was approximately $-335 \mu \varepsilon$ to $+335 \mu \varepsilon$, 
corresponding to an approximate Bragg wavelength shift of $-0.437 \mathrm{~nm}$ to $+0.437 \mathrm{~nm}$. The measured response of FBG 3 to applied bending loads was linear as shown in figure 4.19 , and because it was oriented slightly off the beam axis at approximately -6 degrees, its measured response to applied torsional loads was very small, but still linear as shown in figure 4.20 .

The apparent strain measured by FBG 4 is in agreement within $2 \%$ and a difference of 4 micro-strain of the theoretical axial strain component as shown in Table 4.5. The range of axial strain applied to this sensor was approximately $-450 \mu \varepsilon$ to $+250 \mu \varepsilon$, corresponding to an approximate Bragg wavelength shift of $-0.553 \mathrm{~nm}$ to $+0.307 \mathrm{~nm}$. The measured response of FBG 4 to applied bending loads was linear as shown in figure 4.21 , and because it was oriented at approximately 49 degrees, its measured response to applied torsional loads was also linear as shown in figure 4.22 .

The apparent strain measured by FBG 5 is in agreement within $6 \%$ and a difference of 5 micro-strain of the theoretical axial strain component as shown in Table 4.5. The range of axial strain applied to this sensor was approximately $-90 \mu \varepsilon$ to $+90 \mu \varepsilon$, corresponding to an approximate Bragg wavelength shift of $-0.110 \mathrm{~nm}$ to $+0.110 \mathrm{~nm}$. The measured response of FBG 5 to applied bending loads was linear as shown in figure 4.23 , and because it was oriented at approximately 89.5 degrees, its measured response to applied torsional loads was negligible as shown in figure 4.24 .

The apparent strain measured by FBG 6 is in agreement within $8 \%$ and a difference of 25 micro-strain of the theoretical axial strain component as shown in Table 4.5. The range of axial strain applied to this sensor was approximately $-350 \mu \varepsilon$ to $+350 \mu \varepsilon$, 
corresponding to an approximate Bragg wavelength shift of $-0.433 \mathrm{~nm}$ to $+0.433 \mathrm{~nm}$. The measured response of FBG 6 to applied bending loads was linear as shown in figure 4.25 , and because it was oriented at approximately 2 degrees, its measured response to applied torsional loads was negligible as shown in figure 4.26 .

The apparent strain measured by FBG 7 is in agreement within $5 \%$ and a difference of 20 micro-strain of the theoretical axial strain component as shown in Table 4.5. The range of axial strain applied to this sensor was approximately $-470 \mu \varepsilon$ to $+470 \mu \varepsilon$, corresponding to an approximate Bragg wavelength shift of $-0.577 \mathrm{~nm}$ to $+0.577 \mathrm{~nm}$. The measured response of FBG 7 to applied bending loads was linear as shown in figure 4.27 , and because it was oriented at approximately 43 degrees, its measured response to applied torsional loads was also linear as shown in figure 4.28 .

The apparent strain measured by FBG 8 is in agreement within $20 \%$ and a difference of 19 micro-strain of the theoretical axial strain component as shown in Table 4.5. The range of axial strain applied to this sensor was approximately $-100 \mu \varepsilon$ to $+100 \mu \varepsilon$, corresponding to an approximate Bragg wavelength shift of $-0.124 \mathrm{~nm}$ to $+0.124 \mathrm{~nm}$. The measured response of FBG 8 to applied bending loads was linear as shown in figure 4.29 , and because it was oriented at approximately 89.5 degrees, its measured response to applied torsional loads was negligible as shown in figure 4.30 .

The strain range achieved using the fibreglass specimen was lower than originally planned due to a larger than desired thickness of the top and bottom surface walls. In addition, the elastic modulus of the material was found to be significantly higher than the published value. 


\subsection{Discussion of Results and Errors}

Each FBG sensor was shown in Section 4.4 to respond linearly in proportion to bending and torsional loads as was expected for their measured orientations. For the most part, there was reasonable numerical agreement between the apparent strain and the theoretical axial strain component; thus showing that equation 2.7 predicts with reasonable accuracy the strain induced wavelength shift of an FBG adhered to a structure. However, during the last set of experiments, using FBG 6, FBG 7, FBG 8 and the cyanoacrylate adhesive, there was a noticeable difference in the agreement of the strain results as shown in Table 4.5. The source of this error was believed to be an increased transverse sensitivity of the FBG sensors due to use of the cyanoacrylate adhesive. Before examining this issue, a brief review of other possible sources of error is in order to establish some quantitative estimates of their contribution to the total disagreement. Error quantities estimated will be those typical of a $1550 \mathrm{~nm}$ centre wavelength FBG sensor.

\subsubsection{Data Collection and Processing}

Processing the data sets using the centre wavelength algorithm described in section 4.3 was found to reduce signal alignment errors to within approximately $\pm 0.5 \mathrm{pm}$ and can account for an error of approximately $\pm 0.4 \mu \varepsilon$. The SWS-OMNI system resolution of $\pm 2 \mathrm{pm}$ can account for a strain error of approximately $\pm 1.6 \mu \varepsilon$ within any data set no matter how accurately it is processed. Data collection and processing can thus account for a total strain error of approximately $\pm 2.0 \mu \varepsilon$. 


\subsubsection{Thermal Effects}

Changes to ambient temperatures were observed by calculating the centre wavelength shifts between the zero load data sets recorded at the beginning and end of each measurement series. The greatest shift observed among all series was approximately $5 \mathrm{pm}$ and according to equation 2.7 can account for a maximum strain error of approximately $\pm 4.1 \mu \varepsilon$. Figure 4.14 shows that depending on which test specimen the sensor is installed, this error equates to no more than an ambient temperature change of $\pm 0.4^{\circ} \mathrm{C}$ during any series of data measurements. Localized temperature fluctuations would not be able to account for any noticeable and consistent deviation of the data series trend from theory, as this would imply a consistent source of error. By observing a data series consisting entirely of zero load sets, it was found that the localized temperature fluctuations can be slightly larger than the measured ambient temperature changes, and would thus increase the error by approximately $\pm 1.5 \mu \varepsilon$. Thermal effects during these experiments are therefore estimated to account for a total strain error of approximately $\pm 5.6 \mu \varepsilon$, showing that the CLLIPS laboratory environment was thermally stable within an approximate range of $\pm 0.55^{\circ} \mathrm{C}$.

\subsubsection{Position and Alignment}

The position of each sensor was confirmed to be accurate within $\pm 1.0 \mathrm{~mm}$ as listed in Table 4.2 by measuring their relative position to the rosettes using a set of Vernier calipers. The resulting strain error is at most $\pm 0.16 \%$, corresponding to an extremely small error of $\pm 0.6 \mu \varepsilon, \pm 0.8 \mu \varepsilon$, and $\pm 0.2 \mu \varepsilon$ for FBG 6 , FBG 7 , and FBG 8 respectively. 
A slight misalignment of a sensor from its orientation as listed in Table 4.2 could account for strain values to consistently measure above or below the theoretically predicted values during bending; however, this would cause an increased disagreement between the measured values observed during combined bending and torsion. By varying the measured orientations of a sensor, an improvement of approximately $\pm 2.0 \mu \varepsilon$ could be observed before other values would exceed this disagreement. The error due to misalignment is also bound by Mohr's circle and cannot account for a sensor measuring strain values exceeding the limits established by the principal strains as was the case with FBG 8 shown in figures 4.29 and 4.30 .

\subsubsection{Summary of Experimental Errors}

By combining the strain error estimates, an average strain error of approximately $\pm 10.5 \mu \varepsilon$ was estimated, primarily attributed to thermal sources. This magnitude is close and consistent with the deviations of FBG sensors 1 - 5 noted in Tables 4.4 and 4.5 . After accounting for this error, the remaining unaccounted for errors of FBG 6 , FBG 7 and FBG 8 are approximately $-4.5 \%,+2.3 \%$ and $+9.9 \%$ respectively. The error of sensor 7 is acceptably low considering the theoretical solution agreed less with the rosette measurements during combined bending and torsion. The error of sensor 6 may have been caused by bond degradation, thus reading consistently lower than the theoretical value. The error of sensor 8 however can only be attributed to an increased transverse sensitivity in the sensors caused by the use of cyanoacrylate adhesive. A quick review of 
the strain optic-relationship will reveal where this transverse strain sensitivity was previously unaccounted for.

\subsection{Transverse Sensitivity of FBG Sensors}

The relationships shown in equation 2.7 and equation 2.8 given by Kersey et al. (1997) are reduced forms of the strain-optic relationship and do not directly include a transverse strain component acting on the FBG sensor. These equations assume that any diametral contraction or expansion of the sensor is caused only by the Poisson effect and the axial strain component as can be seen in equation 2.6. This formulation would be valid for sensors that do not have any mechanical constraints in their transverse directions; for sensors significantly constrained by an adhesive or embedded in a laminate, equation 2.6 should be modified to include the average transverse strain acting on the FBG sensor in place the Poisson term as shown by equation 4.2 .

$$
\frac{\Delta \lambda_{B}}{\lambda_{B}}=\left\{\varepsilon_{a x}-\left(\frac{n_{\text {corr }}^{2}}{2}\right)\left[\varepsilon_{a x} P_{12}+\left(\frac{\varepsilon_{T 2}+\varepsilon_{T 3}}{2}\right)\left(P_{11}+P_{12}\right)\right]\right\}
$$

Equation 4.2 is confirmed by Prabhugoud and Peters (2006) to represent the strain-optic response of a FBG sensor under multi-axis strain fields. It can be rearranged to take the familiar form for a strain gauge with axial and transverse sensitivity factors as shown by equation 4.3 , where $\varepsilon_{1}$ is the axial strain component, and $\varepsilon_{2}$ and $\varepsilon_{3}$ are the transverse strain components of the FBG sensor.

$$
\frac{\Delta \lambda_{B}}{\lambda_{B}}=\left(1-\left(\frac{n_{\text {core }}^{2}}{2}\right) P_{12}\right) \varepsilon_{1 f}+\left(\frac{n_{\text {core }}^{2}}{2}\right)\left(-P_{11}-P_{12}\right)\left(\frac{\varepsilon_{2 f}+\varepsilon_{3 f}}{2}\right)
$$


In order to clearly distinguish the different sets of strains and material parameters used within this section, the following subscripts will be used: ' $f$ ' will denote those relating to the 'fibre' or sensor; ' $a$ ' will denote those relating to the 'adhesive'; and ' $l$ ' will denote those relating to the lamina. Directional subscripts ' 1 ', '2', and ' 3 ' are also used and correspond to the axial, in-plane transverse, and out-of-plane transverse directions respectively.

Substituting the appropriate numerical coefficients into equation 4.3 , the strain optic relationship for a FBG sensor can be simplified to be as shown by equation 4.4 having an axial strain sensitivity factor of +0.732 and a transverse sensitivity factor of -0.388 .

$$
\frac{\Delta \lambda_{B}}{\lambda_{B}}=(0.732) \varepsilon_{1 f}+(-0.388)\left(\frac{\varepsilon_{2 f}+\varepsilon_{3 f}}{2}\right)
$$

It may be more convenient to separate the transverse strain components should there be a significant difference in the directional constraint, each having an equal sensitivity factor of -0.194 as shown by equation 4.5 , where $\varepsilon_{2 f}$ is the in-plane transverse strain component and $\varepsilon_{3 f}$ is the out-of-plane transverse strain component.

$$
\frac{\Delta \lambda_{B}}{\lambda_{B}}=(0.732) \varepsilon_{1 f}+(-0.194) \varepsilon_{2 f}+(-0.194) \varepsilon_{3 f}
$$

Equation 4.5 relates the strain of the sensor to a shift in Bragg wavelength. The problem remains to relate the strains seen in the sensor to the unrestrained global strains in the adhesive, or body in which the sensor is embedded. This requires a transfer matrix denoted by $\left[T_{f a}\right]$ in equation 4.6 , which can only be derived for a particular sensor-host 
situation knowing the boundary conditions of the problem, the material constants of each, and whether the interaction is linear elastic or not.

$$
\left[\begin{array}{l}
\varepsilon_{1 f} \\
\varepsilon_{2 f} \\
\varepsilon_{3 f}
\end{array}\right]=\left[T_{f a}\right]\left[\begin{array}{l}
\varepsilon_{1 a} \\
\varepsilon_{2 a} \\
\varepsilon_{3 a}
\end{array}\right]
$$

\subsubsection{Transverse Sensitivity Behaviour Observed in Experimental Results}

Two simple observations that could account for the lack of transverse sensitivity observed by FBG sensors 1 - 5 are use of epoxy/hardener instead of the cyanoacrylate adhesive, and the geometric difference between their cured profiles as shown in figure 4.31. The rudimentary difference in their geometries shows that the epoxy mounted sensors are not effectively constrained in either transverse direction, whereas the cyanoacrylate mounted sensors are considerably constrained in the horizontal direction. The lack of significant transverse constraint on the epoxy sensors implies a near stressfree state in the transverse plane as shown in figure 4.31 and therefore any diametral strains in the sensors would be primarily attributed to the Poisson effect and axial loads. These are nearly the conditions by which equation 4.2 would revert to the form of equation 2.6 , thereby validating the previously presented agreement of experimental results for FBG sensors 1 - 5 with equation 2.7 .

As an example, a transfer matrix $\left[T_{f a}\right]$ will be estimated for FBG 8 by making several simplifying assumptions regarding the sensor interaction with the cyanoacrylate adhesive. The sensor is assumed to undergo the same axial displacement as the host material, restrained by the adhesive in the horizontal plane and 'free' in the vertical plane. 
In reality, of course, it is partly restrained in the vertical plane; nevertheless, plane stress assumption is made in that direction for simplification.

The transverse stress at the sensor-adhesive interface is caused by a disagreement in the unrestrained transverse deformations of each body, which is their natural transverse strains neglecting the presence of the other. For the sensor, the natural strain corresponds to the Poisson effect due to axial loading, and for the adhesive corresponds to the strains caused in it by the structure. By static equilibrium, the normal stress at the interface in each must be equal in magnitude with the other and is therefore derived as shown by equation 4.7 which is null when their natural transverse strains agree.

$$
\sigma_{2}=\left(\varepsilon_{2 a_{0}}-\varepsilon_{2 f_{0}}\right)\left(\frac{E_{a} \times E_{f}}{E_{a}+E_{f}}\right)
$$

Applying Hooke's law, the approximated transfer matrix between the natural adhesive strains and the strain components in the FBG sensor for this particular situation is derived as equation 4.8 , where the relationships have been approximated to the first order by assuming $v_{f}^{2}$ and higher order terms to be negligible in magnitude.

$$
\left[\begin{array}{c}
\varepsilon_{1 f} \\
\varepsilon_{2 f} \\
\varepsilon_{3 f}
\end{array}\right]=\left[\begin{array}{ccc}
1 & 0 \\
\left(-v_{f}\right)\left(1-\left(\frac{E_{a}}{E_{a}+E_{f}}\right)\right) & \left(\frac{E_{a}}{E_{a}+E_{f}}\right) & 0 \\
\left(-v_{f}\right) & \left(-v_{f}\right)\left(\frac{E_{a}}{E_{a}+E_{f}}\right) & 0
\end{array}\right]\left[\begin{array}{l}
\varepsilon_{1 a} \\
\varepsilon_{2 a} \\
\varepsilon_{3 a}
\end{array}\right]
$$


Substituting the appropriate material constants of the fibre and adhesive listed in Table 4.1 into equation 4.8 , the transfer matrix is numerically reduced as shown by equation 4.9.

$$
\left[T_{f a}\right]=\left[\begin{array}{ccc}
1 & 0 & 0 \\
-0.16\left(1-\left(\frac{6}{6+70}\right)\right) & \left(\frac{6}{6+70}\right) & 0 \\
-0.16 & -0.16\left(\frac{6}{6+70}\right) & 0
\end{array}\right]=\left[\begin{array}{ccc}
1 & 0 & 0 \\
-0.147 & 0.079 & 0 \\
-0.16 & -0.013 & 0
\end{array}\right]
$$

The adhesive is assumed to be displacement constrained to the surface of the test specimen; the in-plane strains of the adhesive will thus be the same as those in the specimen and can be oriented to the sensor direction by applying the strain transformation equations $3.18-3.20$. The unrestrained components of strain in the adhesive for FBG 8 are as shown in equation 4.10 in micro-strain units, where the $\varepsilon_{3 a}$ transverse strain is dependant on the Poisson ratios of the adhesive and the test specimen, but is not required in the analysis as it will have no effect on the end result in this case.

$$
\left[\begin{array}{c}
\varepsilon_{1 a} \\
\varepsilon_{2 a} \\
\varepsilon_{3 a}
\end{array}\right]=\left[\begin{array}{c}
80.96 \\
-344.65 \\
\varepsilon_{3 a}
\end{array}\right]_{F B G 8}
$$

The wavelength shift is determined by combining equation $4.10,4.9,4.5$, and the centre wavelength of FBG 8 as listed in Table 4.2 and is found to be $107.0 \mathrm{pm}$. This value is $7 \%$ higher than the shift predicted using equation 2.7 of $100.2 \mathrm{pm}$ and agrees closer to the actual measured value which was reduced from $123.2 \mathrm{pm}$ to $110.0 \mathrm{pm}$ after accounting for other sources of error. The phenomenon of transverse sensitivity is 
therefore shown to be a logical explanation for the experimentally observed measurements of FBG 8 being consistently higher than the theoretical and rosette values.

\subsubsection{Transverse Sensitivity of Embedded Sensors in a Laminate}

When embedding sensors into a laminate, the transverse interaction of the sensor with the host material is considerably more complex than the example presented in Section 4.6.1. Although the experimental work carried out in this study did not include laminates, it is nevertheless useful to discuss briefly this issue here. A sensor at an angled orientation to laminate fibres would form a small resin pocket around it as shown in figure 4.32, whose size, shape and symmetry are dependant on the relative orientations of the sensor to the adjacent laminae. These defects pose a significant structural threat to thinner laminates; in contrast, Skontorp (2002) showed that a sensor installed in parallel with the unidirectional fibres caused almost negligible detriment. The variable boundary conditions of these resin pockets surrounding the sensor in the transverse plane and along its axis make it difficult to accurately predict the interaction of the sensor with the host material. This issue will not be further addressed here; it could be more effectively investigated using numerical methods, such as the boundary element or finite element methods.

By considering the simpler case when a sensor is installed in parallel with unidirectional fibres of a laminate, an estimate of the transverse interaction between a FBG sensor and a lamina of properties similar to the laminate can be made. The estimate assumes the lamina is transversely isotropic; that the sensor is completely and elastically bound within the lamina; that the sensor undergoes the same axial strain as the lamina; 
and that its presence neither significantly reinforces nor weakens the lamina. The estimated transfer matrix, given by equation 4.11 , was derived and reduced keeping only the first order $v_{f}$ terms similar to equation 4.9 , where the lamina transverse modulus is $E_{2 l}$ and the undisturbed direct strains in the lamina are $\varepsilon_{1 l}, \varepsilon_{2 l}, \varepsilon_{3 l}$.

$$
\left[\begin{array}{l}
\varepsilon_{1 f} \\
\varepsilon_{2 f} \\
\varepsilon_{3 f}
\end{array}\right]=\left[\begin{array}{ccc}
1 & 0 \\
\left(-v_{f}\right)\left(1-\left(\frac{E_{2 l}}{E_{2 l}+E_{f}}\right)\right) & \left(\frac{E_{2 l}}{E_{2 l}+E_{f}}\right) & \left(-v_{f}\right)\left(\frac{E_{2 l}}{E_{2 l}+E_{f}}\right) \\
\left(-v_{f}\right)\left(1-\left(\frac{E_{2 l}}{E_{2 l}+E_{f}}\right)\right) & \left(-v_{f}\right)\left(\frac{E_{2 l}}{E_{2 l}+E_{f}}\right) & \left(\frac{E_{2 l}}{E_{2 l}+E_{f}}\right)
\end{array}\right]\left[\begin{array}{l}
\varepsilon_{1 l} \\
\varepsilon_{2 l} \\
\varepsilon_{3 l}
\end{array}\right]
$$

The material parameter $k_{E l}$, defined by equation 4.12 , relates the transverse modulus of the lamina and modulus of the sensor, and is a measure of the lamina's ability to influence the transverse strain of the sensor with its own.

$$
k_{E l}=\frac{E_{2 l}}{E_{2 l}+E_{f}}
$$

If $E_{2 l}$ is significantly lower than the sensor modulus, $E_{f}=70.0 \mathrm{GPa}$, the sensor would be virtually unaffected by the transverse strains present in the lamina, whereas if it is significantly higher, the sensor would almost completely 'assume' the lamina strains. Combining equations $4.12,4.11$, and 4.5 , the expected strain-optic response of a transversely sensitive FBG sensor embedded bare into a unidirectional lamina parallel to the fibre axis can thus be approximated as shown by equation 4.13 . 


$$
\frac{\Delta \lambda_{B}}{\lambda_{B}}=\left[\begin{array}{ccc}
0.732+0.388 v_{f}\left(1-k_{E l}\right) & -0.194 k_{E l}\left(1-v_{f}\right) & -0.194 k_{E l}\left(1-v_{f}\right)
\end{array}\right]\left[\begin{array}{l}
\varepsilon_{1 l} \\
\varepsilon_{2 l} \\
\varepsilon_{3 l}
\end{array}\right]
$$

For laminae stacked into thick laminates, such as structural stiffening members, the three direct strains $\varepsilon_{1 l}, \varepsilon_{2 l}, \varepsilon_{3 l}$ in the lamina could be independent, and would thus need to be treated separately. Laminae stacked in thin shell laminates however are assumed to be in plane stress, thereby making the out-of-plane strain $\varepsilon_{3 l}$ dependant on the in-plane strains $\varepsilon_{1 l}, \varepsilon_{2 l}$. Even though the laminae are considered to be in plane stress, their elastic interaction with an embedded sensor in the through-thickness direction should not be excluded. Applying Hooke's Law for a transversely isotropic material and the plane stress condition, the out-of-plane direct strain $\varepsilon_{3 l}$ for a lamina in a thin shell laminate is derived as shown by equation 4.14 , where $v_{22 l}$ and $v_{12 l}$ are the Poisson ratios of the lamina similar to $v_{z t}$ and $v_{t}$ respectively, and $E_{1 l}$ is the lamina modulus in the fibre axis.

$$
\varepsilon_{3 l}=\left[\frac{v_{12 l}+v_{22 l} \cdot v_{12 l}}{v_{12 l}{ }^{2} \cdot E_{2 l} / E_{1 l}-1}\right] \varepsilon_{1 l}+\left[\frac{v_{22 l}+v_{12 l}{ }^{2} \cdot E_{2 l} / E_{1 l}}{v_{12 l}{ }^{2} \cdot E_{2 l} / E_{1 l}-1}\right] \varepsilon_{2 l}=K_{13 l} \cdot \varepsilon_{1 l}+K_{23 l} \cdot \varepsilon_{2 l}
$$

Two coefficients, $K_{13 l}, K_{23 l}$, are defined to represent the contributions of $\varepsilon_{1 l}, \varepsilon_{2 l}$ to the out-of-plane strain of a lamina under plane stress. Substituting equation 4.14 into equation 4.13, the strain-optic relationship of the embedded FBG sensor is reduced into equation 4.15 as a function of only the in-plane direct strains $\varepsilon_{1 l}, \varepsilon_{2 l}$. 


$$
\frac{\Delta \lambda_{B}}{\lambda_{B}}=\begin{gathered}
{\left[0.732+0.388 \cdot v_{f}\left(1-k_{E l}\right)-0.194 \cdot k_{E l}\left(1-v_{f}\right) \cdot K_{13}\right] \cdot \varepsilon_{1 l}} \\
+\left[-0.194 \cdot k_{E l}\left(1-v_{f}\right)-0.194 \cdot k_{E l}\left(1-v_{f}\right) \cdot K_{23}\right] \cdot \varepsilon_{2 l}
\end{gathered}
$$

This equation is of the familiar form presented for electrical resistance strain gauges of equation 3.1, where the axial strain sensitivity factor is given by equation 4.16 , the transverse strain sensitivity factor is given by equation 4.17 .

$$
\begin{aligned}
& F_{a}=\left[0.732+0.388 \cdot v_{f}\left(1-k_{E l}\right)-0.194 \cdot k_{E l}\left(1-v_{f}\right) \cdot K_{13}\right] \\
& F_{t}=\left[-0.194 \cdot k_{E l}\left(1-v_{f}\right)-0.194 \cdot k_{E l}\left(1-v_{f}\right) \cdot K_{23}\right]
\end{aligned}
$$

An 'optical' gauge factor $F_{o p t}$ and transverse sensitivity correction factor $k_{o p t}$ can be defined by equations 4.18 and 4.19 respectively.

$$
\begin{gathered}
F_{o p t}=0.732+0.388 \cdot v_{f}\left(1-k_{E l}\right)-0.194 \cdot k_{E l}\left(1-v_{f}\right)\left[1+K_{13}+K_{23}\right] \\
k_{o p t}=\frac{\left[-0.194 \cdot k_{E}\left(1-v_{f}\right)\left[1+K_{23}\right]\right]}{\left[0.732+0.388 \cdot v_{f}\left(1-k_{E l}\right)-0.194 \cdot k_{E l}\left(1-v_{f}\right) \cdot K_{13}\right]}
\end{gathered}
$$

This allows FBG measurements to be taken as single 'apparent' strains, which can be separated into the true strain components by using equation 4.20 and knowing the ratio of $\varepsilon_{2 l} / \varepsilon_{1 l}$; or alternatively, employing multiple sensors at different orientations and relating their strains by the strain transformation equations $3.18-3.20$, similar to a rosette analysis.

$$
\varepsilon_{o p t}^{\prime}=\frac{\Delta \lambda_{B}}{\lambda_{B}} \frac{1}{F_{o p t}}=\varepsilon_{1 l} \frac{1}{\left(1+k_{o p t}\right)}+\varepsilon_{2 l} \frac{k_{o p t}}{\left(1+k_{o p t}\right)}
$$




\subsection{Applying FBG Sensors to Thin Shell Laminates}

Thin shell laminates are considered to be in a state of plane stress, and as such, according to Hooke's law, only three of the six strain components will be independent, the two inplane direct strains $\varepsilon_{z}, \varepsilon_{x}$, and the in-plane shear strain $\varepsilon_{z x}$. Unless the principal strain orientations are known, three FBG sensor measurements would be needed at a single point in order to characterize the full state of strain in the laminate, each at a different orientation that is known relative to the others.

The simplest solution to this problem would be to deploy three FBG sensors at different levels in the laminate, each at a different orientation. This, however, gives rise to a potential problem. As it was suggested in Section 4.6.2, orienting FBG sensors differently between laminae results in different and difficult to predict sensitivity factors which need to be known for every gauge in order to relate their measurements in a rosette configuration. A simple solution to this problem is to completely embed each FBG sensor into a unidirectional lamina, which are then be oriented in at least three different directions within the laminate thickness. By making the centres of each FBG sensor in the differently oriented laminae coincident through the thickness, the result is to have effectively created a 'stacked' FBG rosette, ensuring that each sensor is measuring the same strain field. To consistently embed sensors into unidirectional laminae, the FBG could be sandwiched between two laminae of equal thickness, creating a thicker lamina.

The relationships presented in Section 4.6 .2 could be used to estimate the sensor sensitivity factors; these estimates could be further calibrated and corrected to their true values by performing a series of simple laminate coupon tests with embedded sensors. 


\subsection{Concluding Remarks}

The experimental tests successfully demonstrated the ability of FBG sensors to measure with high numerical accuracy the strains in the test specimens, even over small ranges. Temperature effects were suspected to be the primary contributor to strain measurement errors, which could be eliminated by the use of a temperature discriminating system or sensor. During the final set of tests, an increased transverse sensitivity was observed in the FBG measurements, and was later attributed to the elastic interaction of the sensor and its host material. When embedding FBG sensors in fibre reinforced laminates, they should be installed parallel to the fibre axis direction in order to exhibit more consistent sensitivity factors, and to pose less threat to the structural integrity of the host. Having verified their strain measurement ability and challenges, it remains to determine how FBG sensors can be efficiently networked and integrated into the SHARCS rotor design, and meaningful data extracted from them. 
Table 4.1 Typical properties of experimental materials used in FBG experiments

\begin{tabular}{llllll}
\hline Property & $\begin{array}{l}\text { Aluminum } \\
\text { Specimen }\end{array}$ & $\begin{array}{l}\text { Fibreglass } \\
\text { Specimen }\end{array}$ & Epoxy/Hardener & Cyanoacrylate & $\begin{array}{l}\text { Ge Doped } \\
\text { Silica } \\
\text { Fibre }\end{array}$ \\
\hline \hline$E_{1}$ & $68.95 \mathrm{GPa}$ & $30.2 \mathrm{GPa}$ & $3.5 \mathrm{GPa}$ & $6.0 \mathrm{GPa}$ & $70.0 \mathrm{GPa}$ \\
$G_{1}$ & $25.92 \mathrm{GPa}$ & $4.1 \mathrm{GPa}$ & $1.35 \mathrm{GPa}$ & $2.22 \mathrm{GPa}$ & $30.2 \mathrm{GPa}$ \\
$v_{1 t}$ & 0.33 & 0.25 & 0.3 & 0.35 & 0.16 \\
$n_{e}$ & N/A & N/A & N/A & N/A & 1.458 \\
$p_{11}$ & N/A & N/A & N/A & N/A & 0.113 \\
$p_{12}$ & N/A & N/A & N/A & N/A & 0.252 \\
\hline
\end{tabular}

Table 4.2 Fibre Bragg grating sensor information

\begin{tabular}{lllllll}
\hline FBG & Specimen & Surface & Adhesive Used & $\begin{array}{l}\mathbf{z} \\
(\mathbf{m m})\end{array}$ & $\begin{array}{l}\text { Orientation } \\
\text { (degrees) }\end{array}$ & $\begin{array}{l}\text { Centre } \\
\text { Wavelength } \\
(\mathbf{n m})\end{array}$ \\
\hline 1 & Aluminum & Top & Epoxy/Hardener & 661 & 1.75 & 1545.171727 \\
2 & Aluminum & Top & Epoxy/Hardener & 613 & -42.0 & 1547.385582 \\
3 & Fibreglass & Top & Epoxy/Hardener & 667 & -6.0 & 1544.714513 \\
4 & Fibreglass & Top & Epoxy/Hardener & 680 & 49.0 & 1546.985424 \\
5 & Fibreglass & Bottom & Epoxy/Hardener & 640 & 89.5 & 1544.642149 \\
6 & Fibreglass & Bottom & Cyanoacrylate & 637 & 2.0 & 1557.100481 \\
7 & Fibreglass & Top & Cyanoacrylate & 610 & 43.0 & 1543.895765 \\
8 & Fibreglass & Top & Cyanoacrylate & 633 & 89.5 & 1557.178893 \\
\hline
\end{tabular}


Table 4.3 Sample of raw optical data

\begin{tabular}{lllll}
\hline $\begin{array}{l}\text { Wavelength } \\
\text { (nm) }\end{array}$ & Transmission & \multicolumn{3}{l}{ Reflection } \\
\cline { 2 - 5 } & $\begin{array}{l}\text { Insertion Loss } \\
\text { (Db) }\end{array}$ & $\begin{array}{l}\text { Group Delay } \\
\text { (s) }\end{array}$ & $\begin{array}{l}\text { Insertion Loss } \\
\text { (Db) }\end{array}$ & $\begin{array}{l}\text { Group Delay } \\
\text { (s) }\end{array}$ \\
\hline 1543.800894 & -7.227310 & 502.99365 & -28.24890 & 1850.51833 \\
1543.803859 & -7.251820 & 505.05346 & -29.54755 & 1850.51603 \\
1543.806823 & -7.368600 & 507.48857 & -28.94098 & 1852.40722 \\
1543.809788 & -7.620670 & 512.60985 & -25.61900 & 1986.75931 \\
1543.812752 & -8.014170 & 507.93015 & -21.69442 & 2143.85351 \\
1543.815717 & -8.542950 & 506.78431 & -19.32384 & 2078.26427 \\
1543.818682 & -9.182540 & 499.76033 & -17.81734 & 2140.33000 \\
1543.821646 & -9.920610 & 495.55047 & -16.73932 & 2176.73726 \\
1543.824611 & -10.67103 & 493.37699 & -15.92085 & 2186.36320 \\
1543.827576 & -11.45869 & 483.34894 & -15.37974 & 2201.20584 \\
1543.830540 & -12.27095 & 480.48622 & -14.95571 & 2212.33189 \\
1543.833505 & -13.04050 & 475.09788 & -14.64231 & 2217.33202 \\
1543.836470 & -13.79808 & 474.90916 & -14.38424 & 2220.58268 \\
1543.839434 & -14.53107 & 474.96822 & -14.24284 & 2226.00144 \\
\hline
\end{tabular}

Table 4.4 FBG sensor measurements on the aluminum beam

\begin{tabular}{llllllll}
\hline \multirow{2}{*}{ FBG } & \multicolumn{2}{l}{ Moment (Nm) } & \multicolumn{2}{c}{ Strain Values (micro-strain) } & \multicolumn{2}{c}{ FBG to Theory } \\
\cline { 2 - 8 } & Bending & Torsional & Theoretical & Rosette & FBG & Diff. & \% Diff. \\
\hline \hline 1 & 328.63 & 0.00 & 903.86 & 889.80 & 911.53 & 7.67 & 0.85 \\
& -328.63 & 0.00 & -903.86 & -903.74 & -898.80 & 5.06 & -0.56 \\
& 328.63 & 88.39 & 887.86 & 883.20 & 900.47 & 12.61 & 1.42 \\
& -328.63 & -88.39 & -887.86 & -893.74 & -899.16 & -11.3 & 1.27 \\
\hline 2 & 304.95 & 0.00 & 339.70 & 332.57 & 336.47 & -3.23 & -0.95 \\
& -304.95 & 0.00 & -339.70 & -341.96 & -352.86 & -13.16 & 3.87 \\
& 304.95 & 88.39 & 641.28 & 644.49 & 628.97 & -12.31 & -1.92 \\
& -304.95 & -88.39 & -641.28 & -651.60 & -638.00 & 3.28 & -0.51 \\
\hline
\end{tabular}


Table 4.5 FBG sensor measurements on the fibreglass beam

\begin{tabular}{|c|c|c|c|c|c|c|c|}
\hline \multirow{2}{*}{ FBG } & \multicolumn{2}{|c|}{ Moment (Nm) } & \multicolumn{3}{|c|}{ Strain Values (micro-strain) } & \multicolumn{2}{|c|}{ FBG to Theory } \\
\hline & Bending & Torsional & Theoretical & Rosette & FBG & Diff. & $\%$ Diff. \\
\hline \multirow[t]{4}{*}{3} & 391.69 & 0.00 & 354.19 & 354.16 & 351.06 & -3.13 & -0.88 \\
\hline & -391.69 & 0.00 & -354.19 & -354.70 & -348.43 & 5.76 & -1.62 \\
\hline & 152.38 & -57.98 & 110.24 & 111.20 & 104.08 & -6.16 & -5.58 \\
\hline & -152.38 & 57.98 & -110.24 & -110.06 & -113.75 & -3.51 & 3.18 \\
\hline \multirow[t]{4}{*}{4} & 399.14 & 0.00 & 104.45 & 103.82 & 106.36 & 1.91 & 1.83 \\
\hline & -399.14 & 0.00 & -104.45 & -104.74 & -103.92 & 0.53 & -0.51 \\
\hline & 399.14 & 59.62 & 241.94 & 242.53 & 241.76 & -0.18 & -0.08 \\
\hline & -399.14 & -149.04 & -448.18 & -445.44 & -451.82 & -3.64 & 0.81 \\
\hline \multirow[t]{4}{*}{5} & 375.39 & 0.00 & 87.57 & 86.30 & 90.22 & 2.65 & 3.03 \\
\hline & -375.39 & 0.00 & -87.57 & -84.80 & -82.88 & 4.69 & -5.36 \\
\hline & 146.04 & -57.98 & 31.85 & 31.35 & 31.61 & -0.24 & -0.75 \\
\hline & -146.04 & 57.98 & -31.85 & -30.57 & -33.61 & -1.76 & 5.52 \\
\hline \multirow[t]{4}{*}{6} & 373.99 & 0.00 & -342.36 & -342.35 & -316.62 & 25.74 & -7.52 \\
\hline & -373.99 & 0.00 & 342.36 & 342.85 & 333.84 & -8.52 & -2.49 \\
\hline & 373.99 & -149.04 & -365.05 & -361.54 & -350.71 & 14.34 & -3.93 \\
\hline & -373.99 & 149.04 & 365.05 & 363.63 & 346.03 & -19.02 & -5.21 \\
\hline \multirow[t]{4}{*}{7} & 358.16 & 0.00 & 136.76 & 136.30 & 135.29 & -1.47 & -1.07 \\
\hline & -358.16 & 0.00 & -136.76 & -137.06 & -142.50 & -5.74 & 4.20 \\
\hline & 358.16 & -149.04 & 447.46 & 437.31 & 466.39 & 18.93 & 4.23 \\
\hline & -358.16 & 149.04 & -447.46 & -432.39 & -468.44 & -20.98 & 4.69 \\
\hline \multirow[t]{4}{*}{8} & 371.20 & 0.00 & -86.59 & -85.33 & -104.75 & -18.16 & 20.97 \\
\hline & -371.20 & 0.00 & 86.59 & 83.85 & 99.90 & 13.31 & 15.36 \\
\hline & 371.20 & -149.04 & -80.96 & -79.68 & -97.44 & -16.48 & 20.36 \\
\hline & -371.20 & 149.04 & 80.96 & 77.71 & 99.50 & 18.54 & 22.90 \\
\hline
\end{tabular}




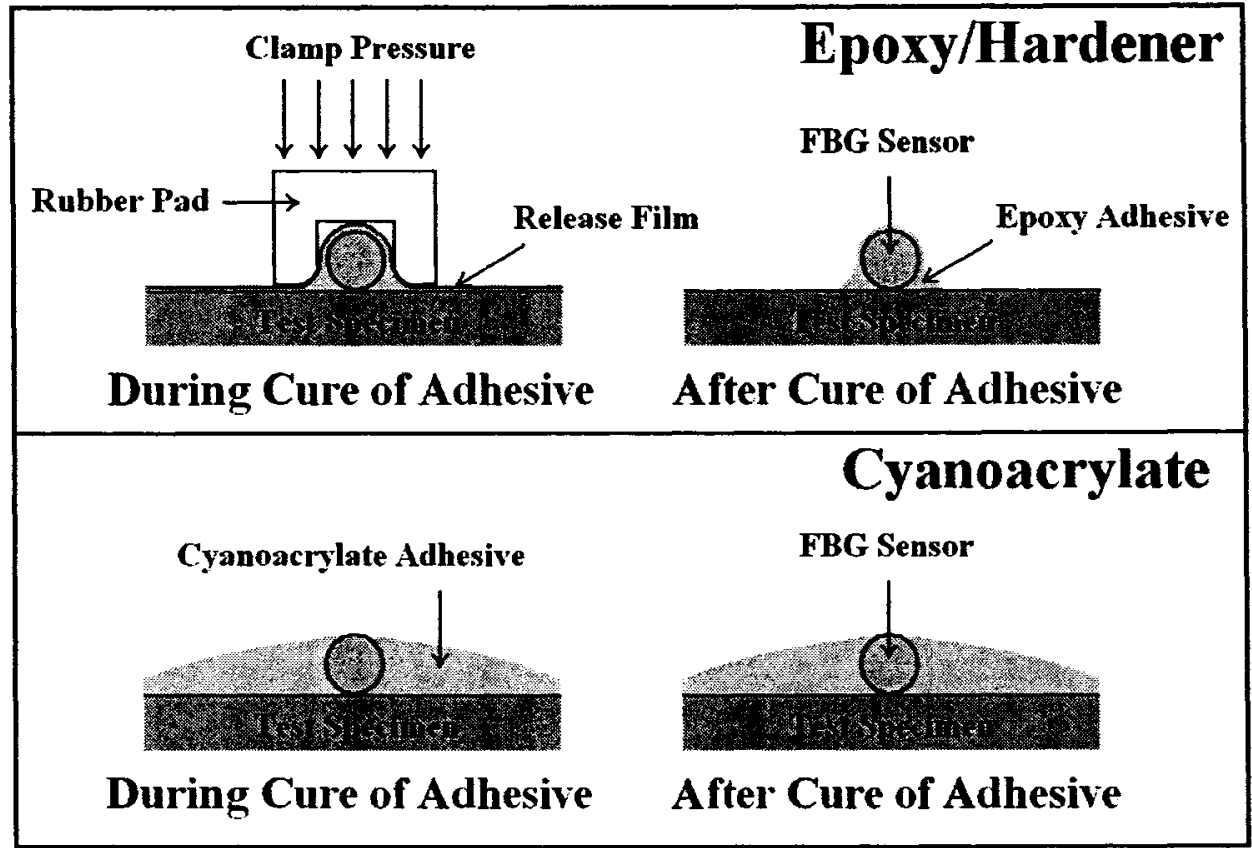

Figure 4.1 Installation of a FBG using different adhesives

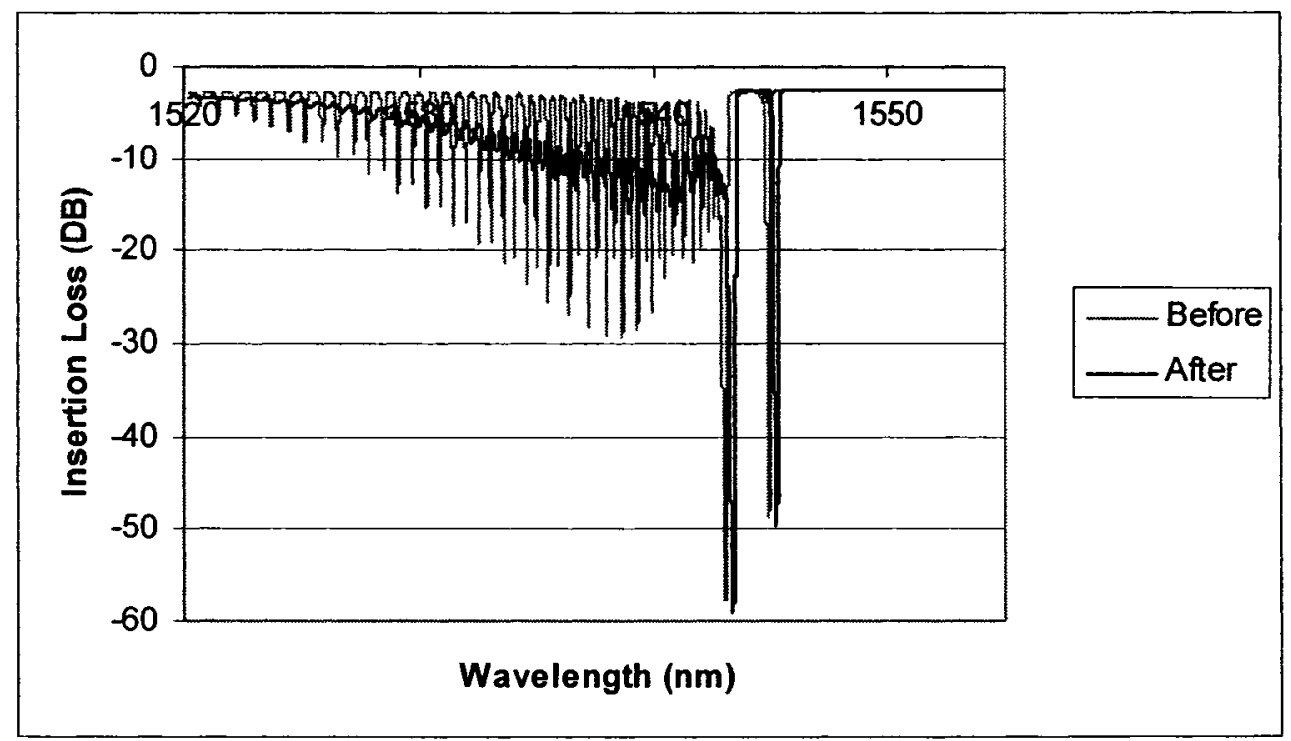

Figure 4.2 Optical signal change of tilted FBG \#1 during installation 


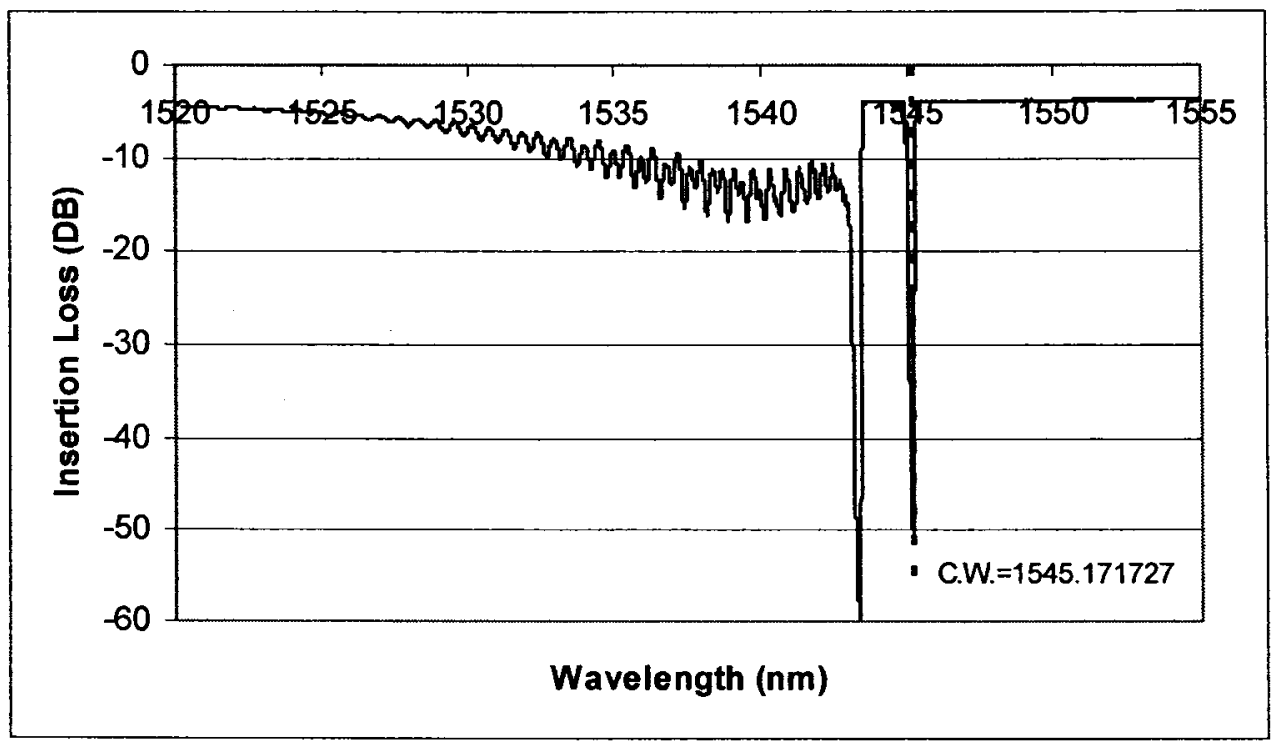

Figure 4.3 Optical signal of FBG \#1 after installation

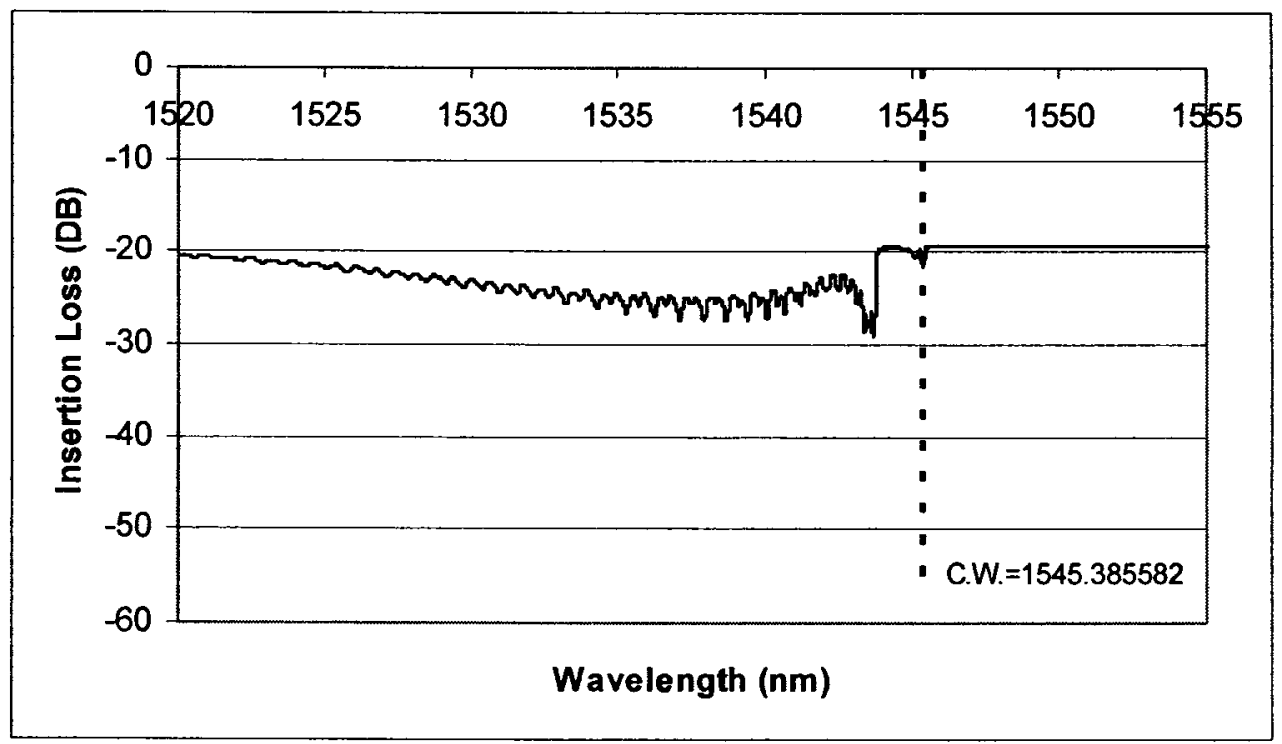

Figure 4.4 Optical signal of FBG \#2 after installation 


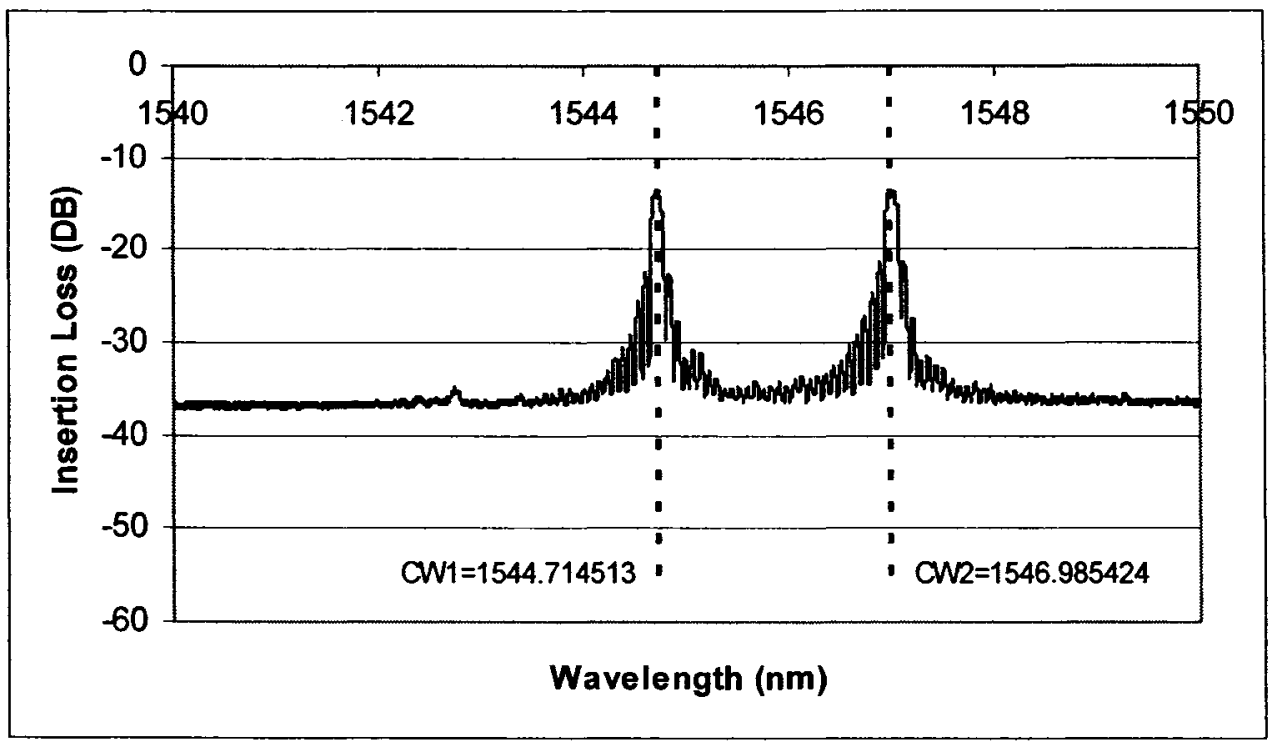

Figure 4.5 Optical signal of FBG \#3 after installation

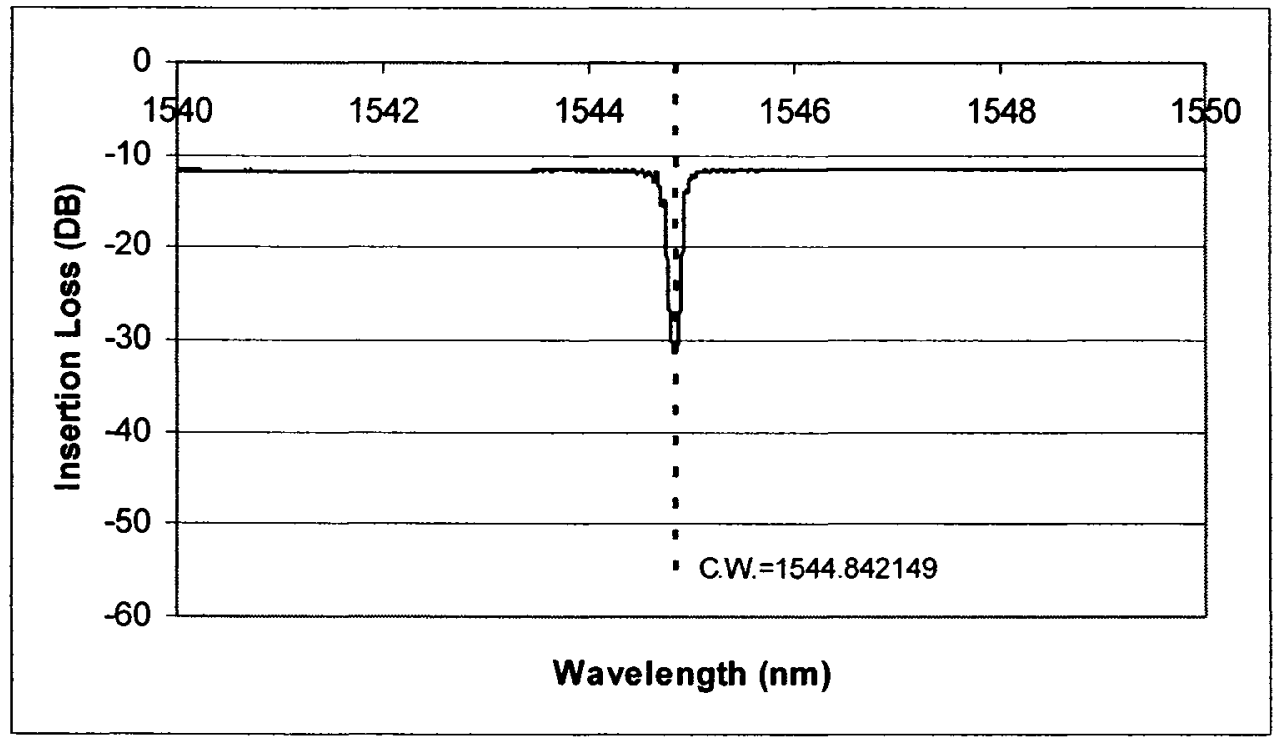

Figure 4.6 Optical signal of FBG \#4 after installation 


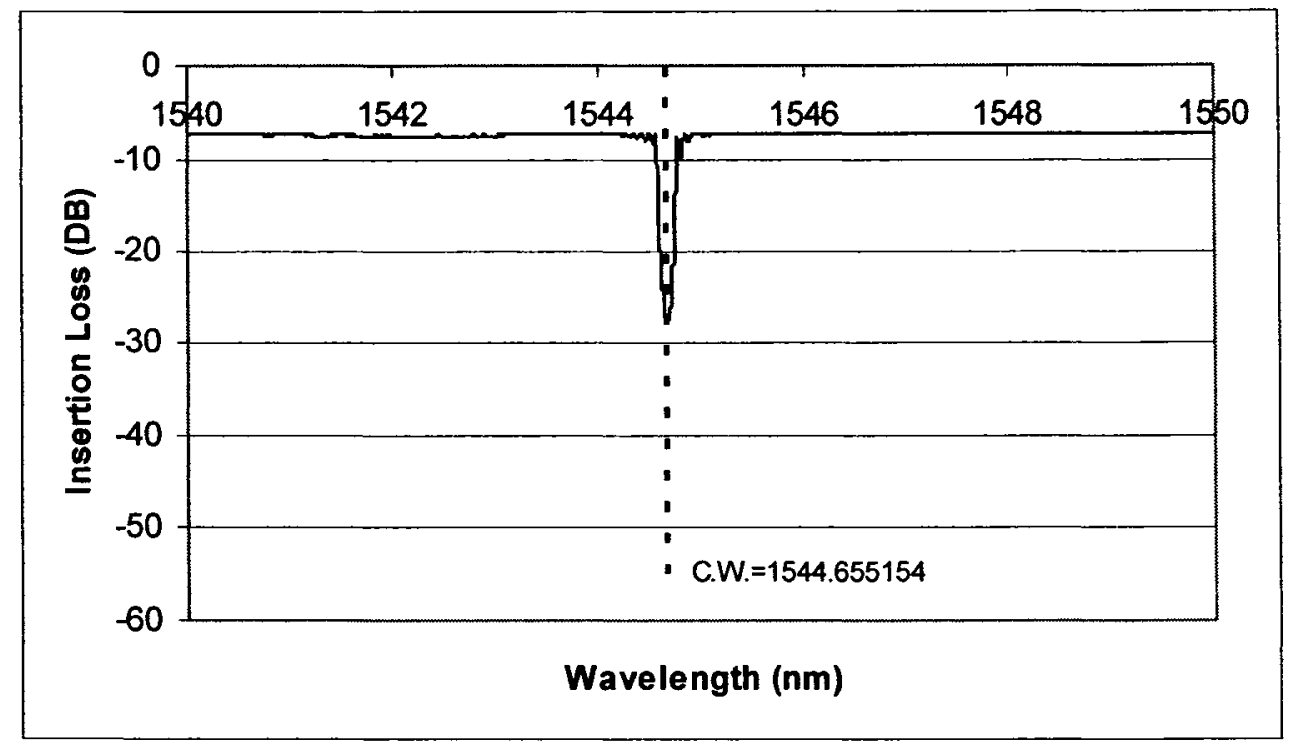

Figure 4.7 Optical signal of FBG \#5 after installation

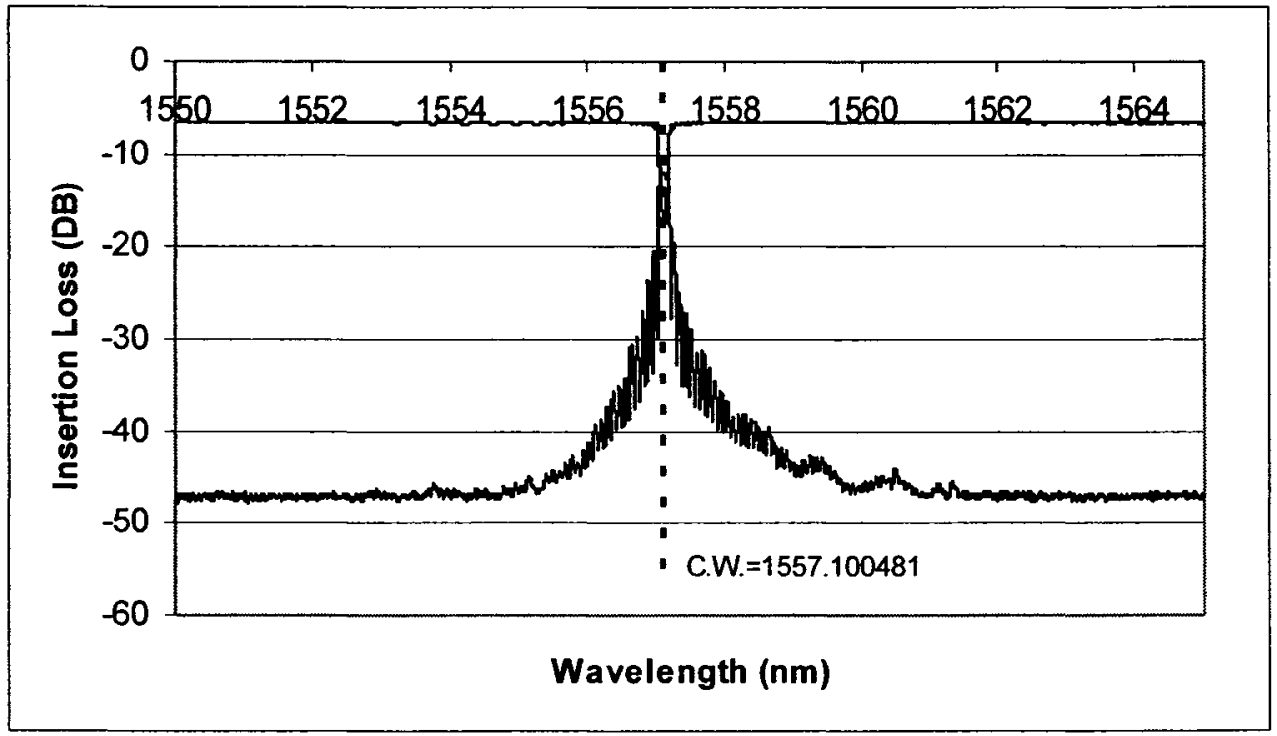

Figure 4.8 Optical signal of FBG \#6 after installation 


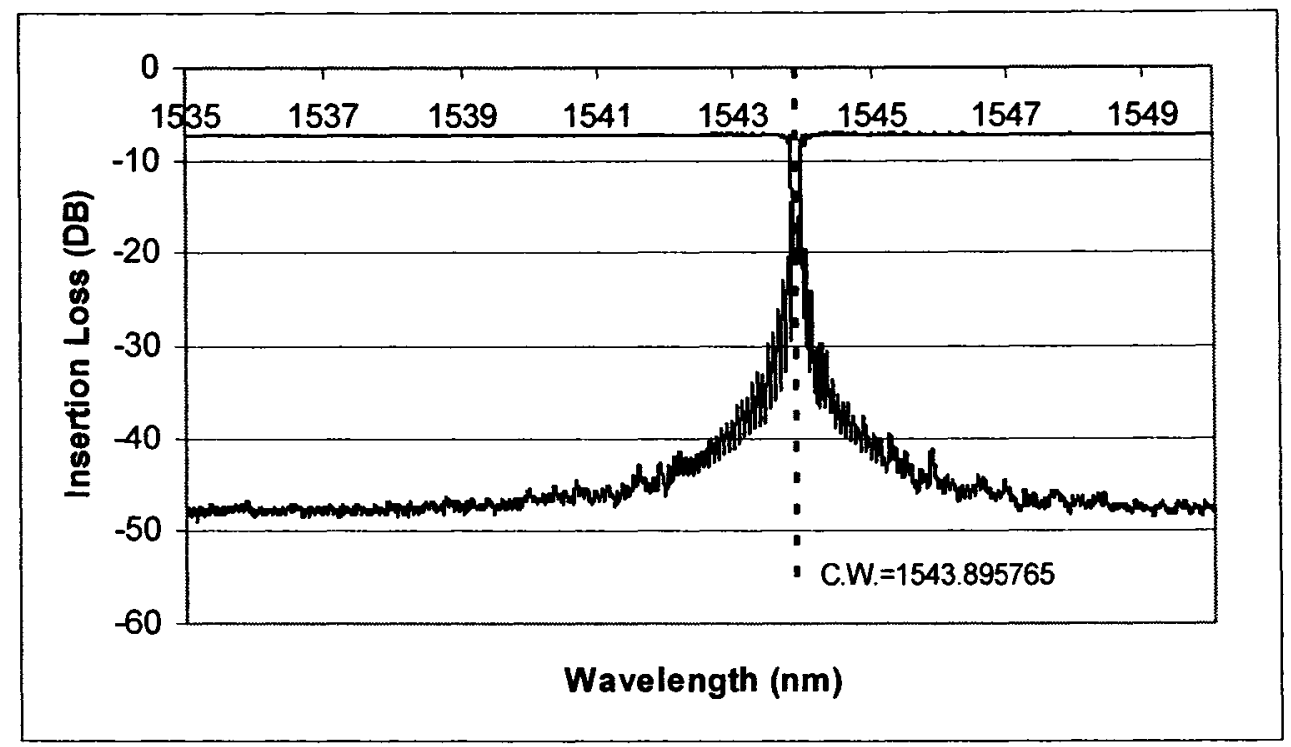

Figure 4.9 Optical signal of FBG \#7 after installation

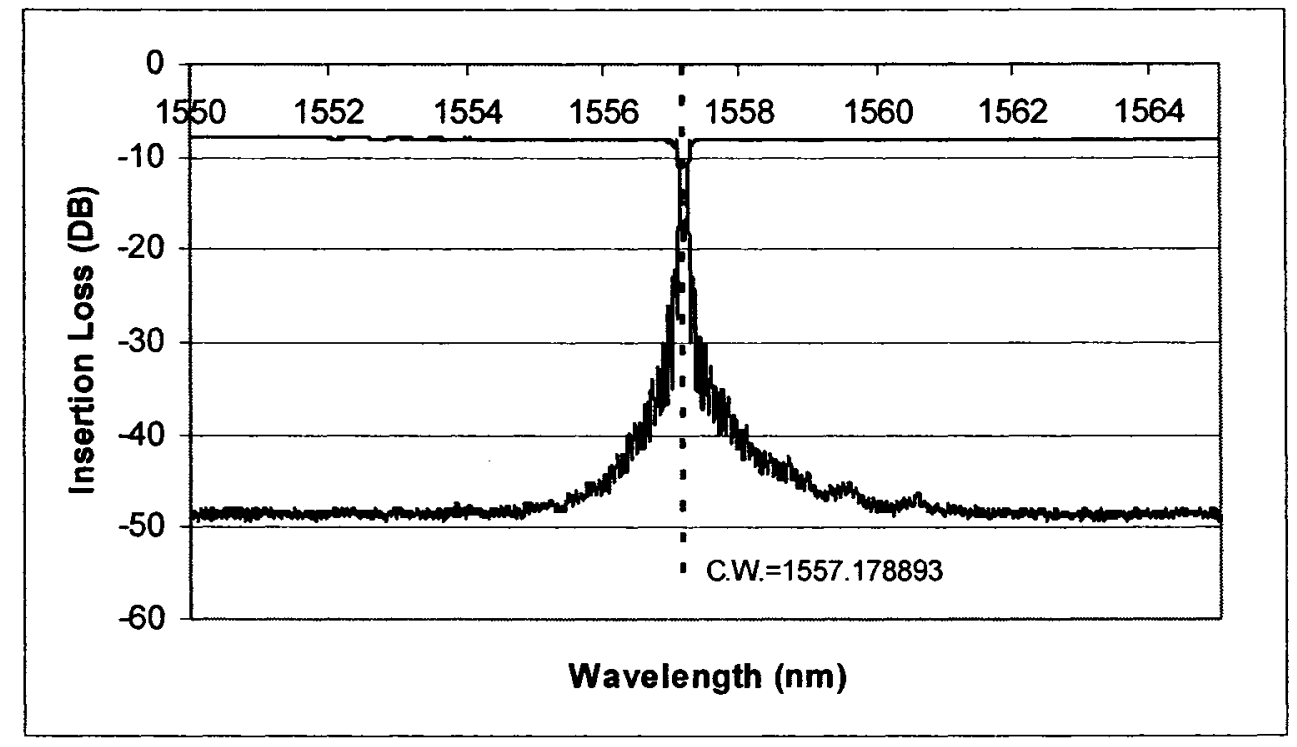

Figure 4.10 Optical signal of FBG $\# 8$ after installation 


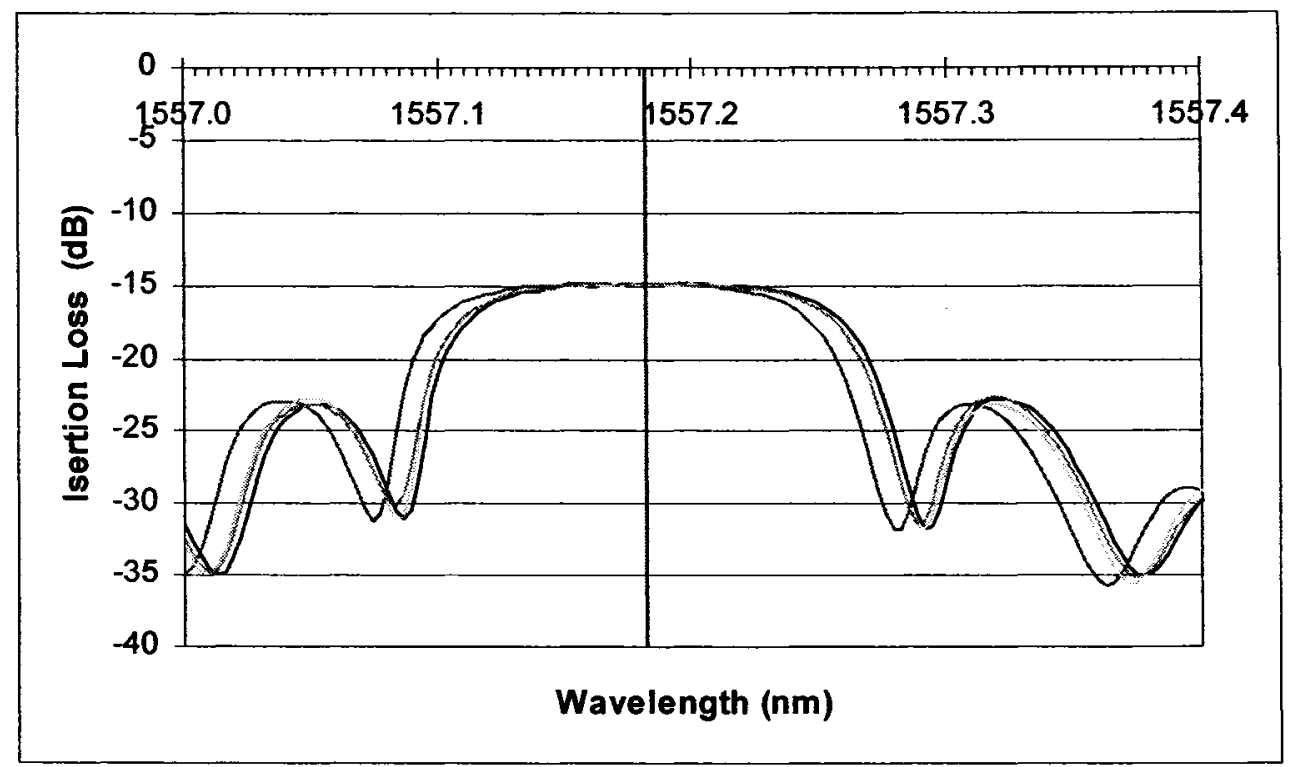

Figure 4.11 FBG reflection signals aligned using maximum insertion loss

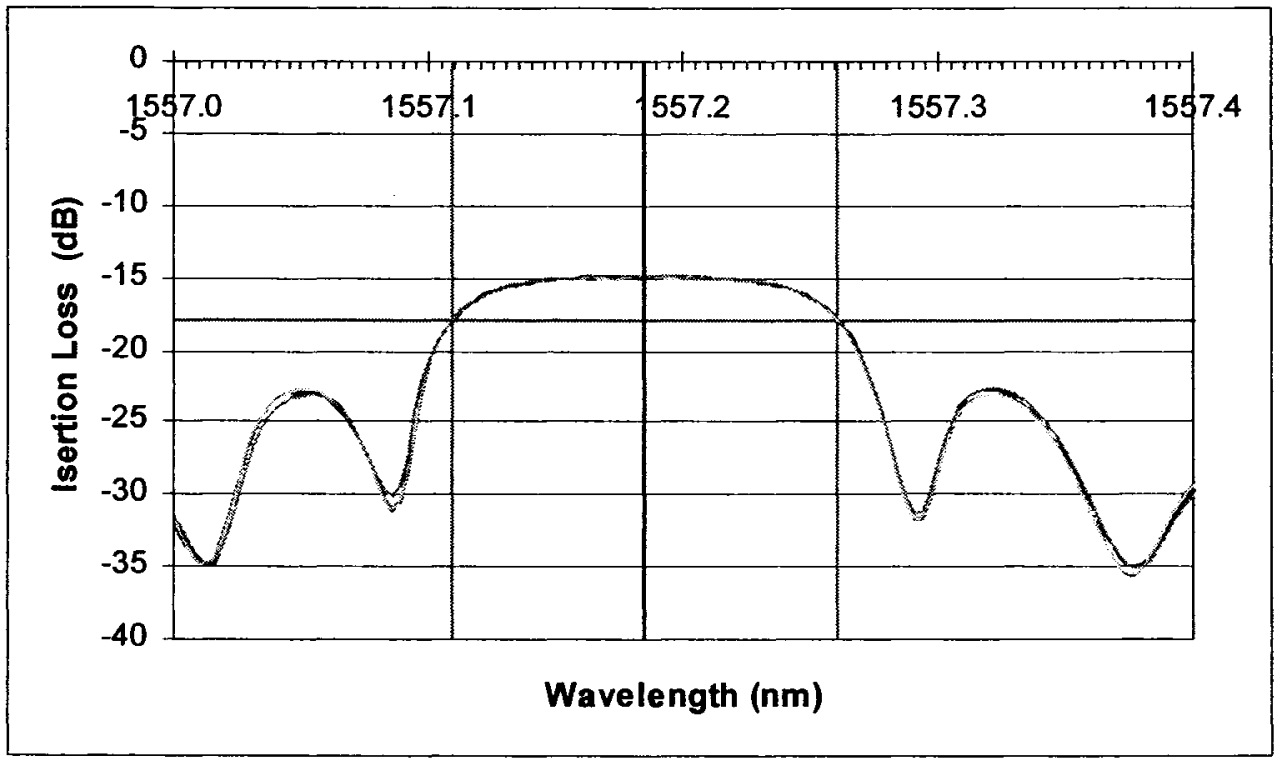

Figure 4.12 FBG reflection signals aligned using centre wavelength 




Figure 4.13 Shift of FBG \#4 optical signal due to applied strain

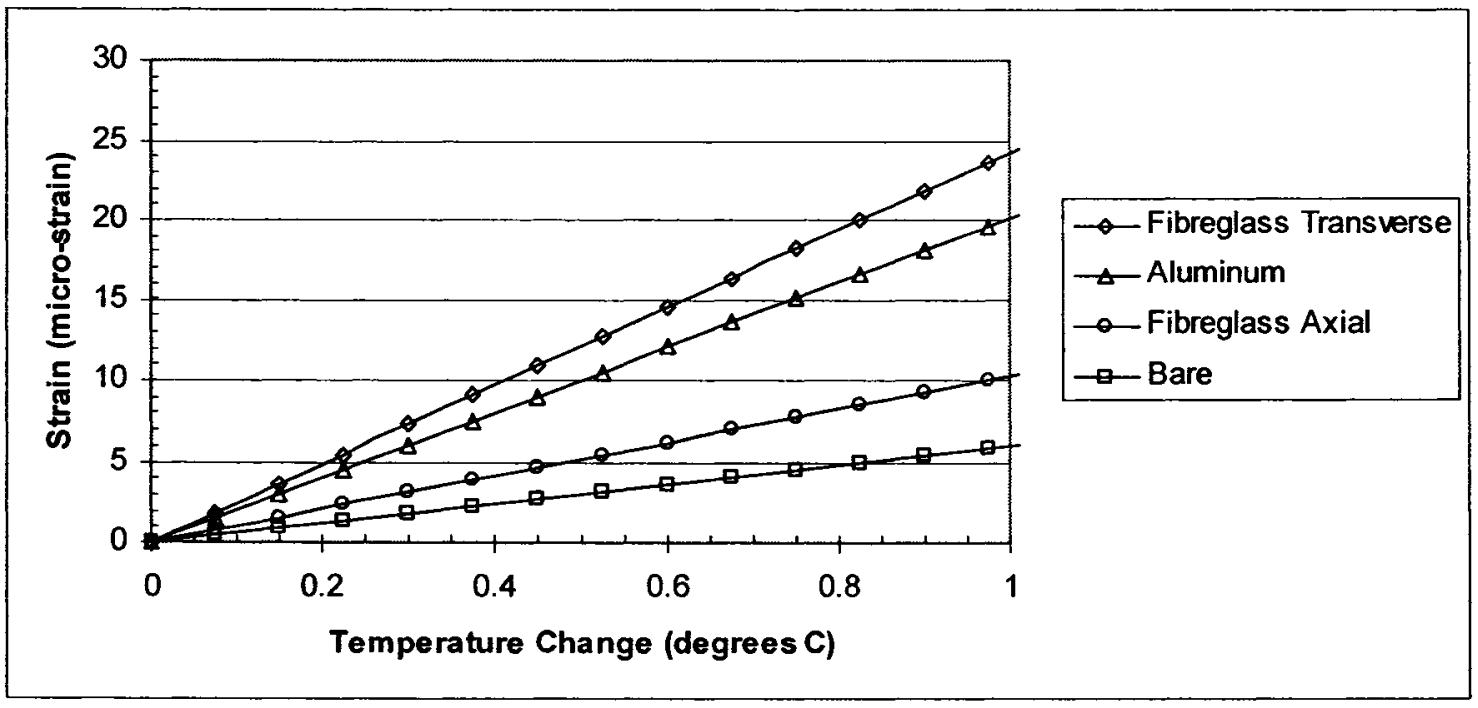

Figure 4.14 Error in FBG strain readings due to thermal sensitivity 


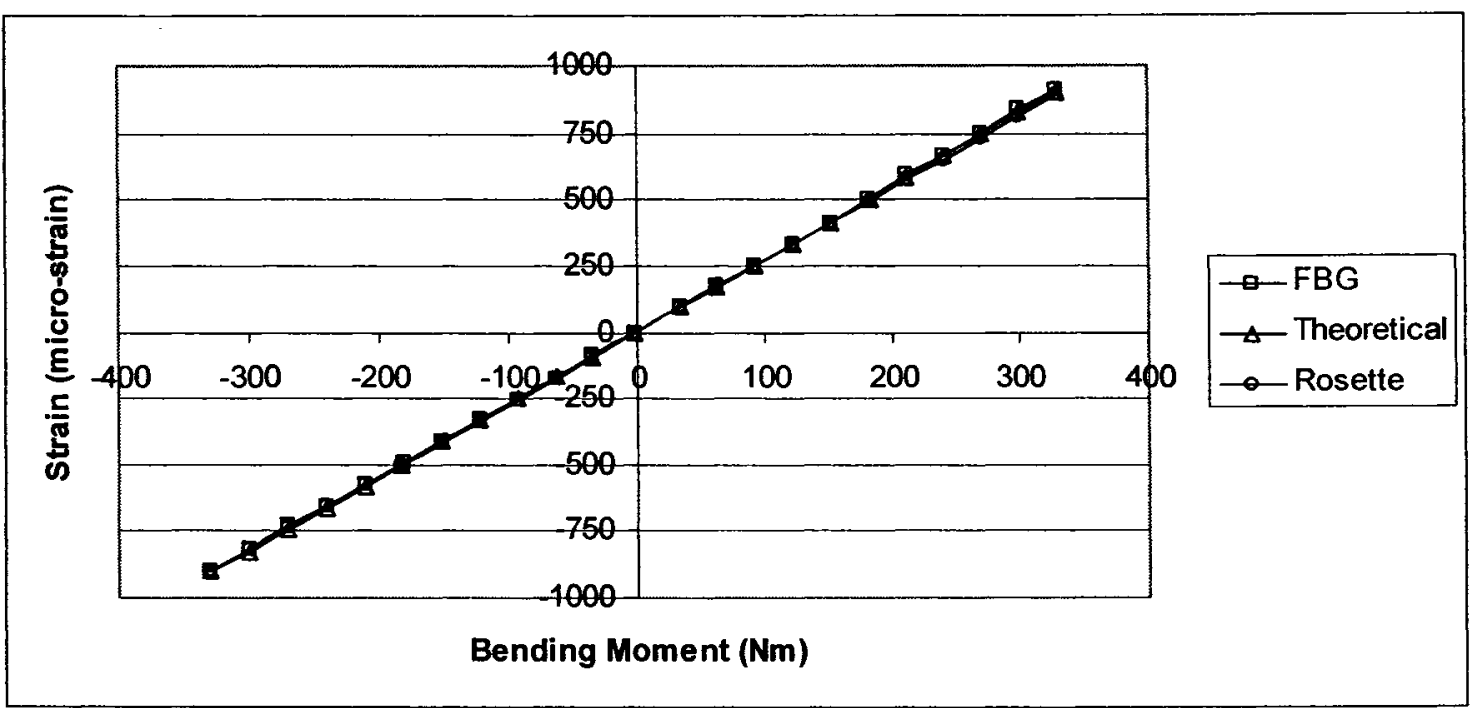

Figure 4.15 Bending results for axial strain on FBG \#1

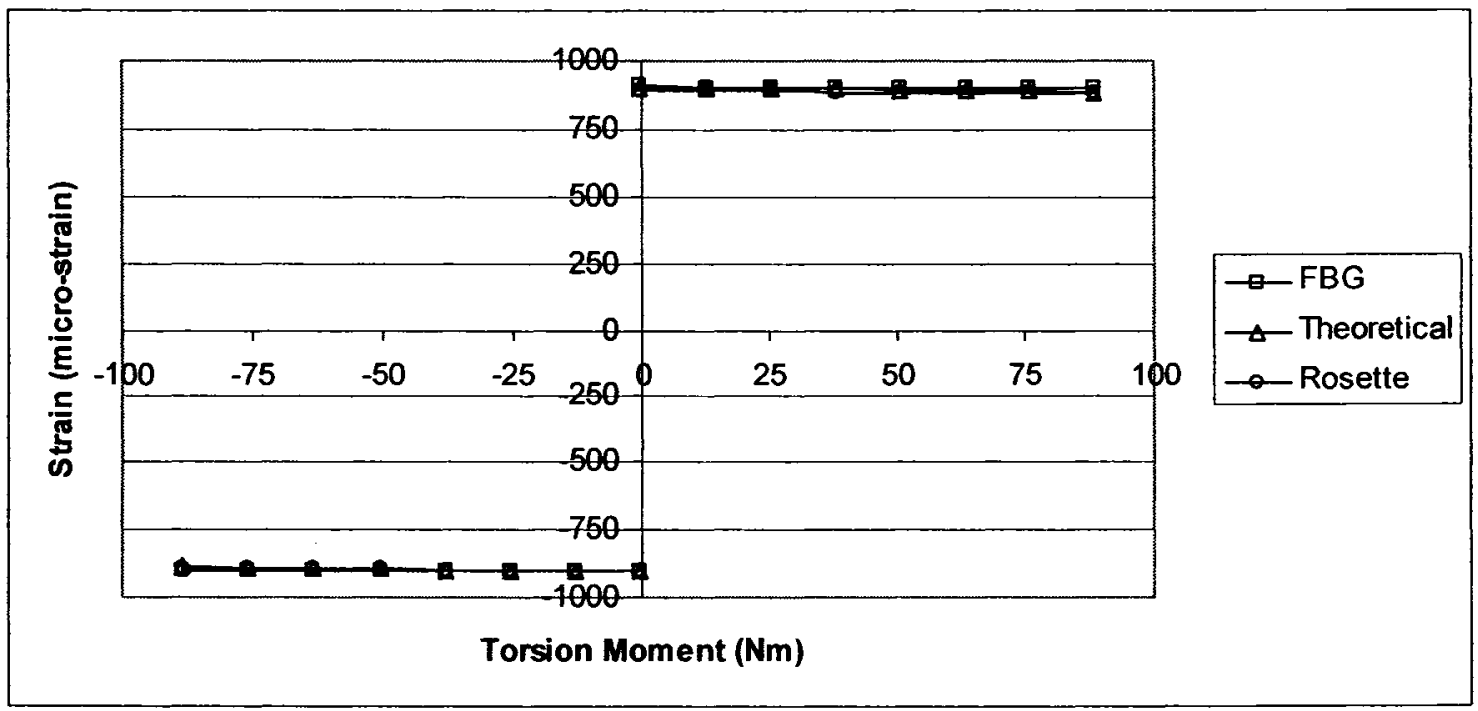

Figure 4.16 Combined bending and torsion results for axial strain on FBG \#1 


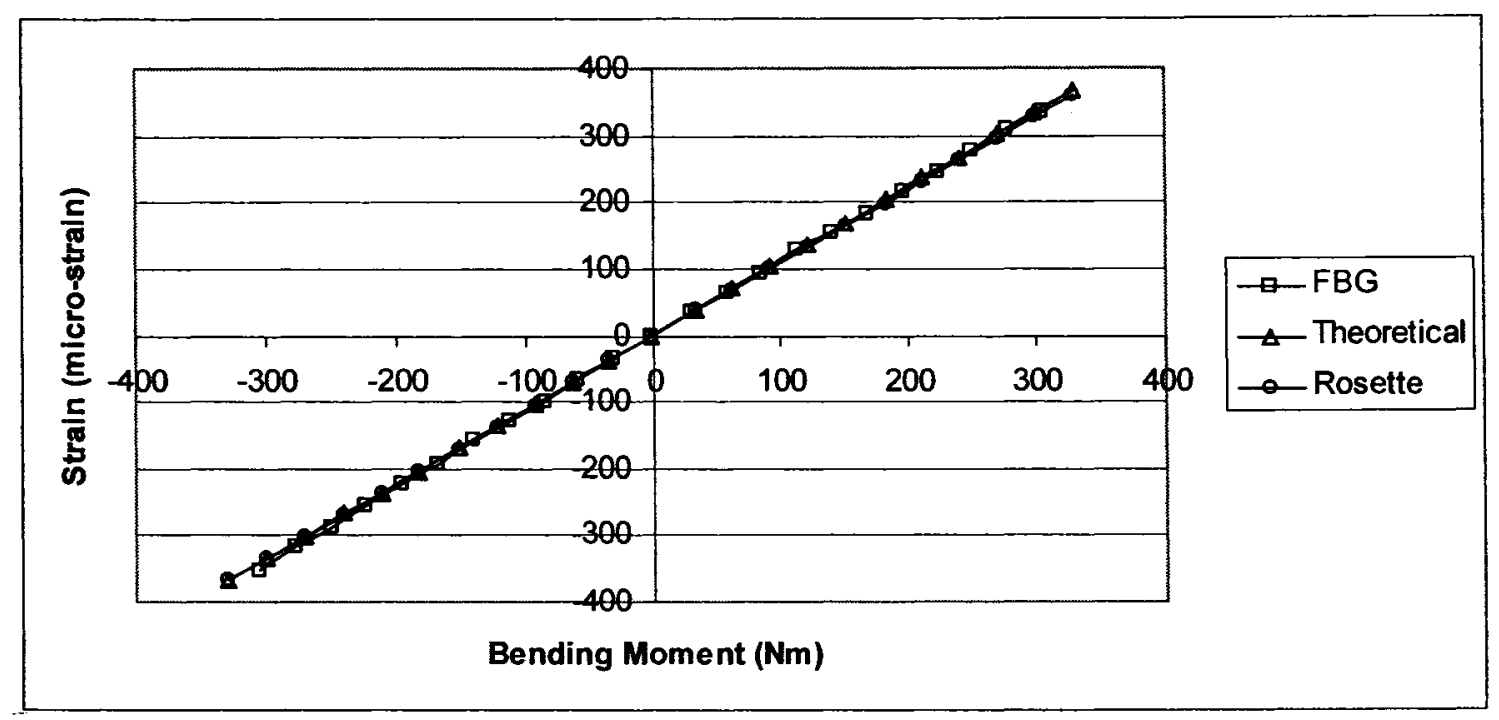

Figure 4.17 Bending results for axial strain on FBG \#2

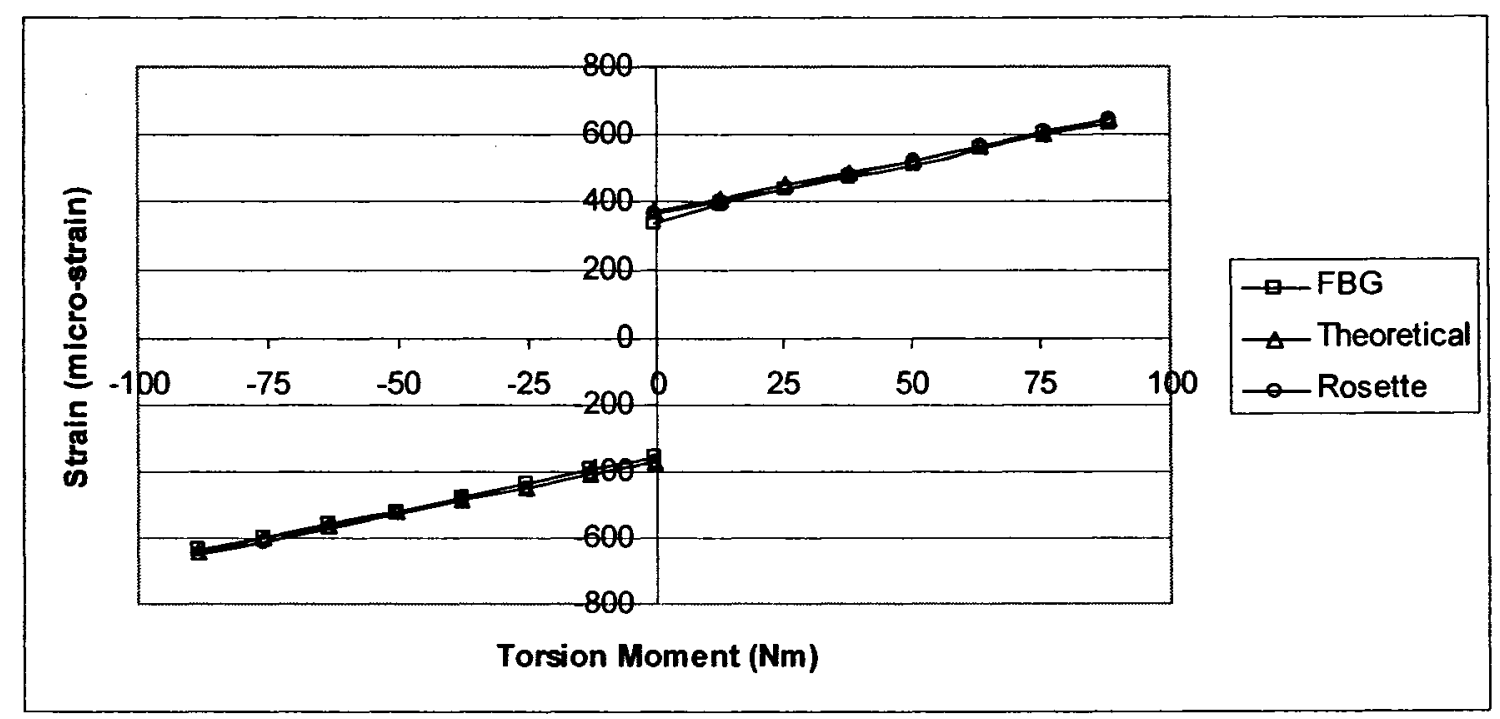

Figure 4.18 Combined bending and torsion results for axial strain on FBG \#2 


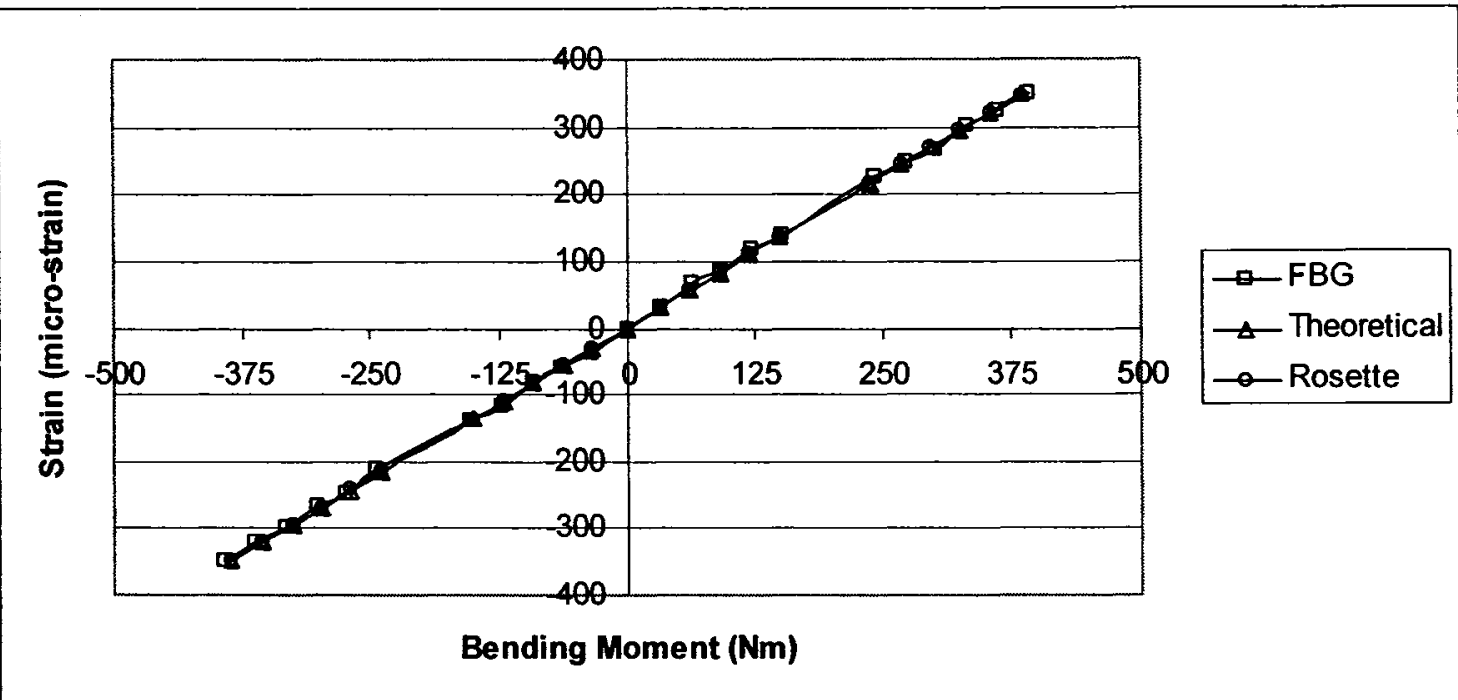

Figure 4.19 Bending results for axial strain on FBG \#3

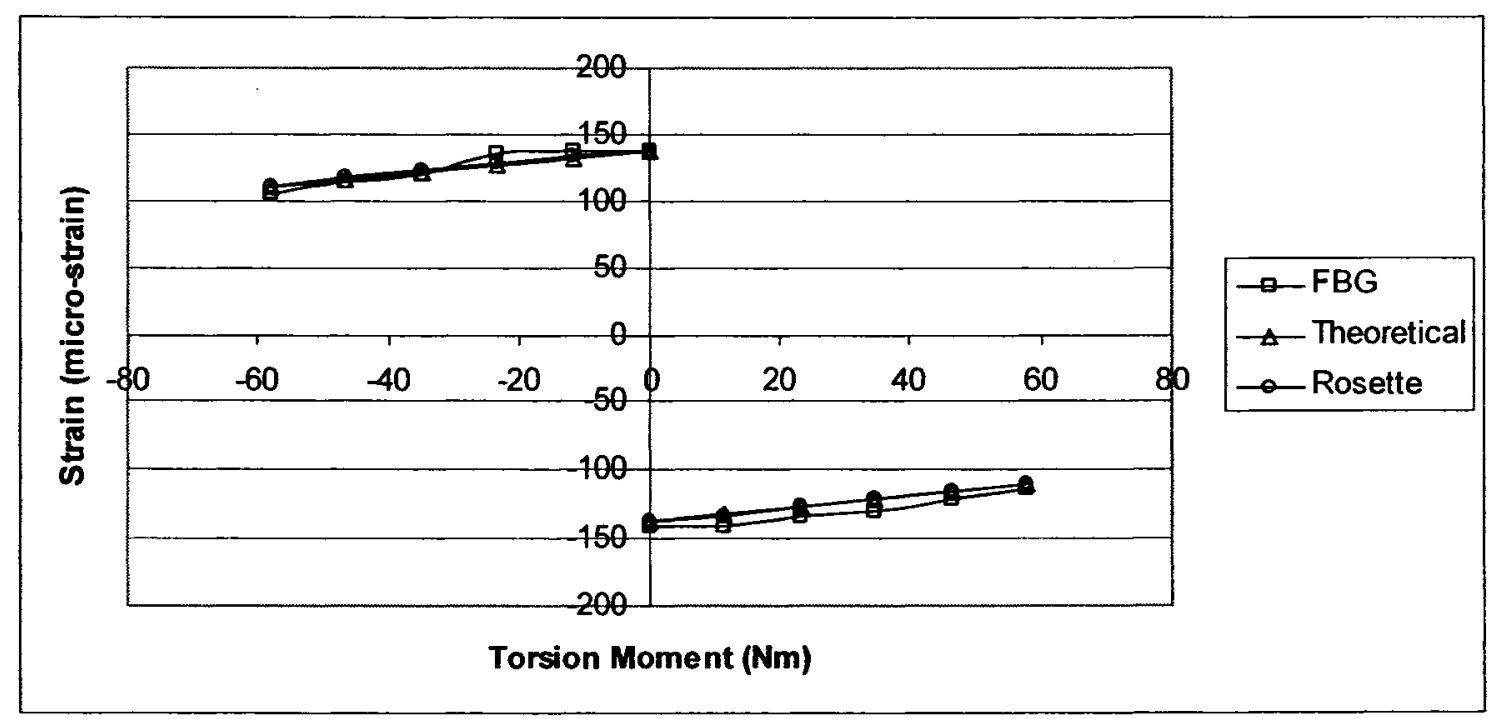

Figure 4.20 Combined bending and torsion results for axial strain on FBG \#3 


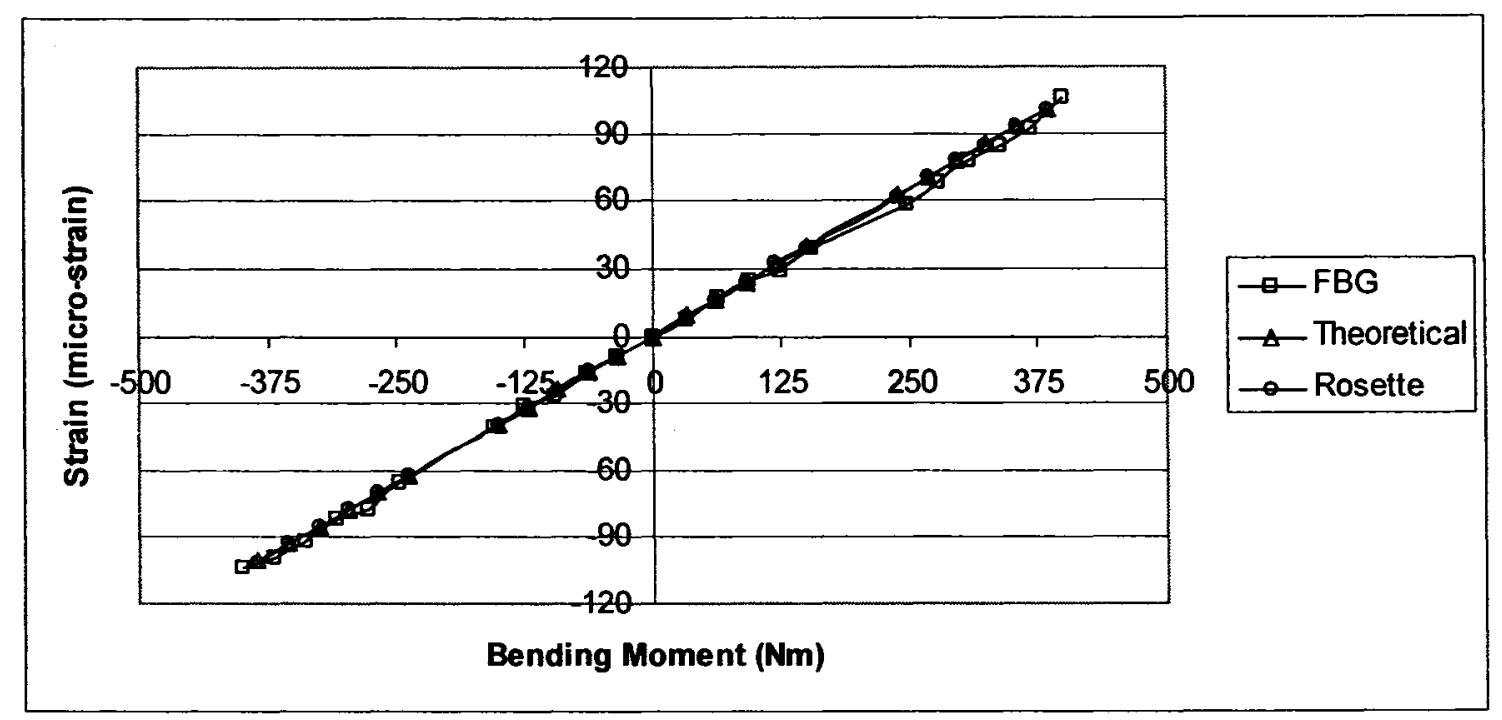

Figure 4.21 Bending results for axial strain on FBG \#4

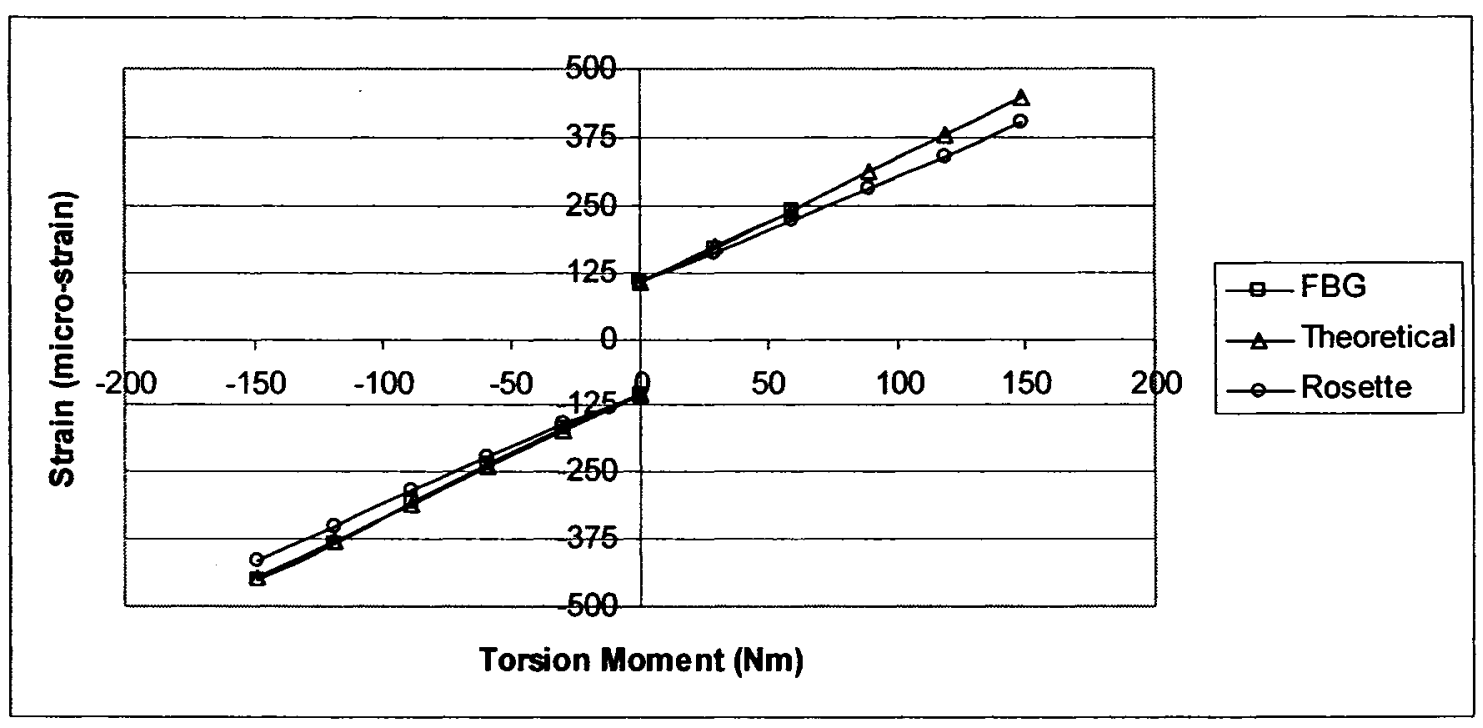

Figure 4.22 Combined bending and torsion results for axial strain on FBG \#4 


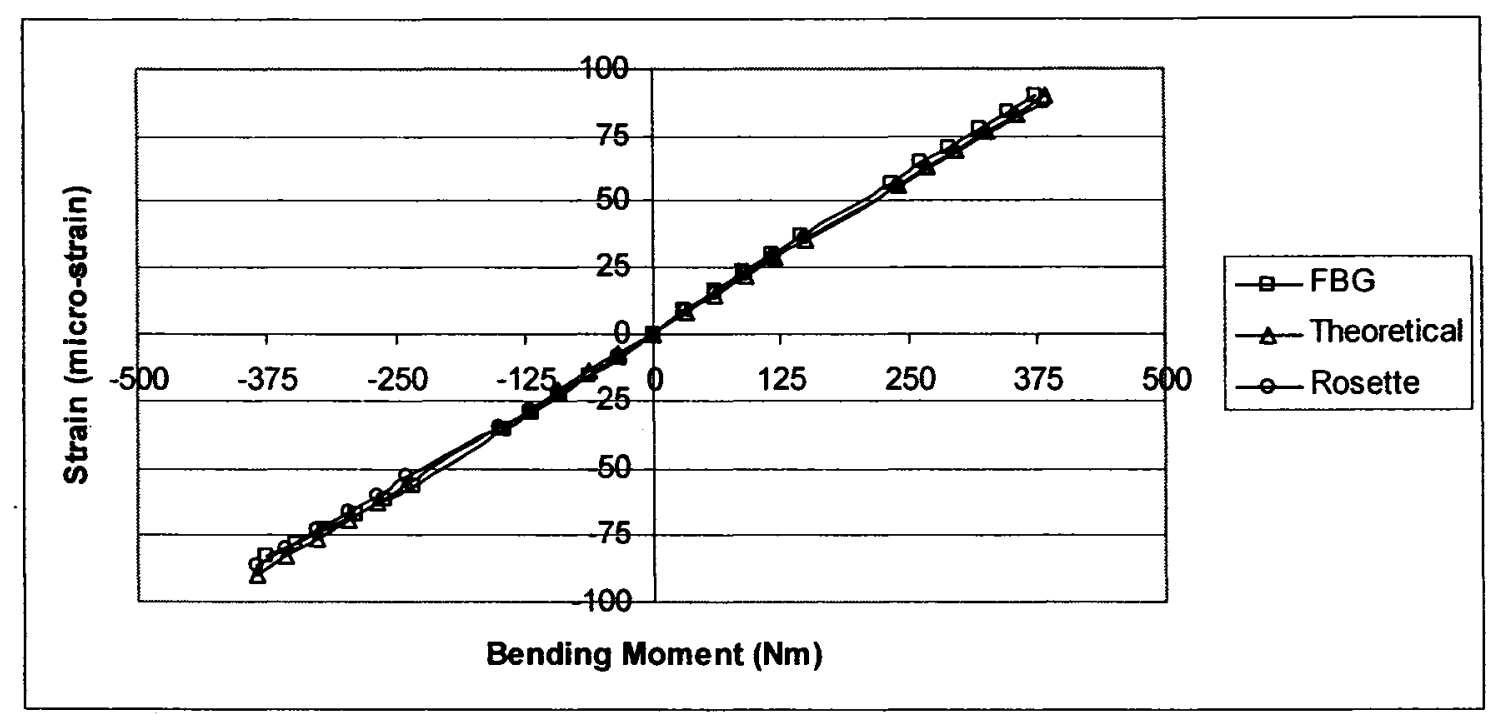

Figure 4.23 Bending results for axial strain on FBG \#5

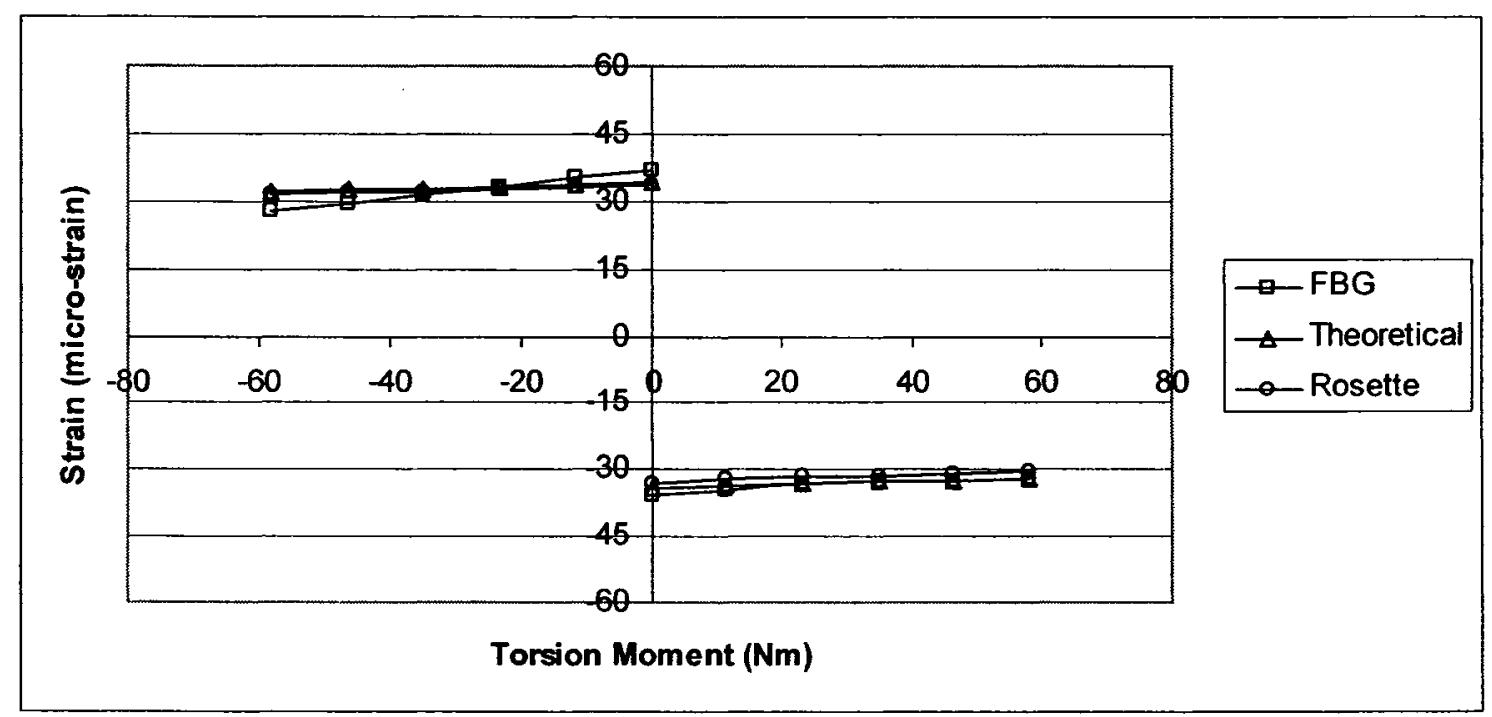

Figure 4.24 Combined bending and torsion results for axial strain on FBG \#5 


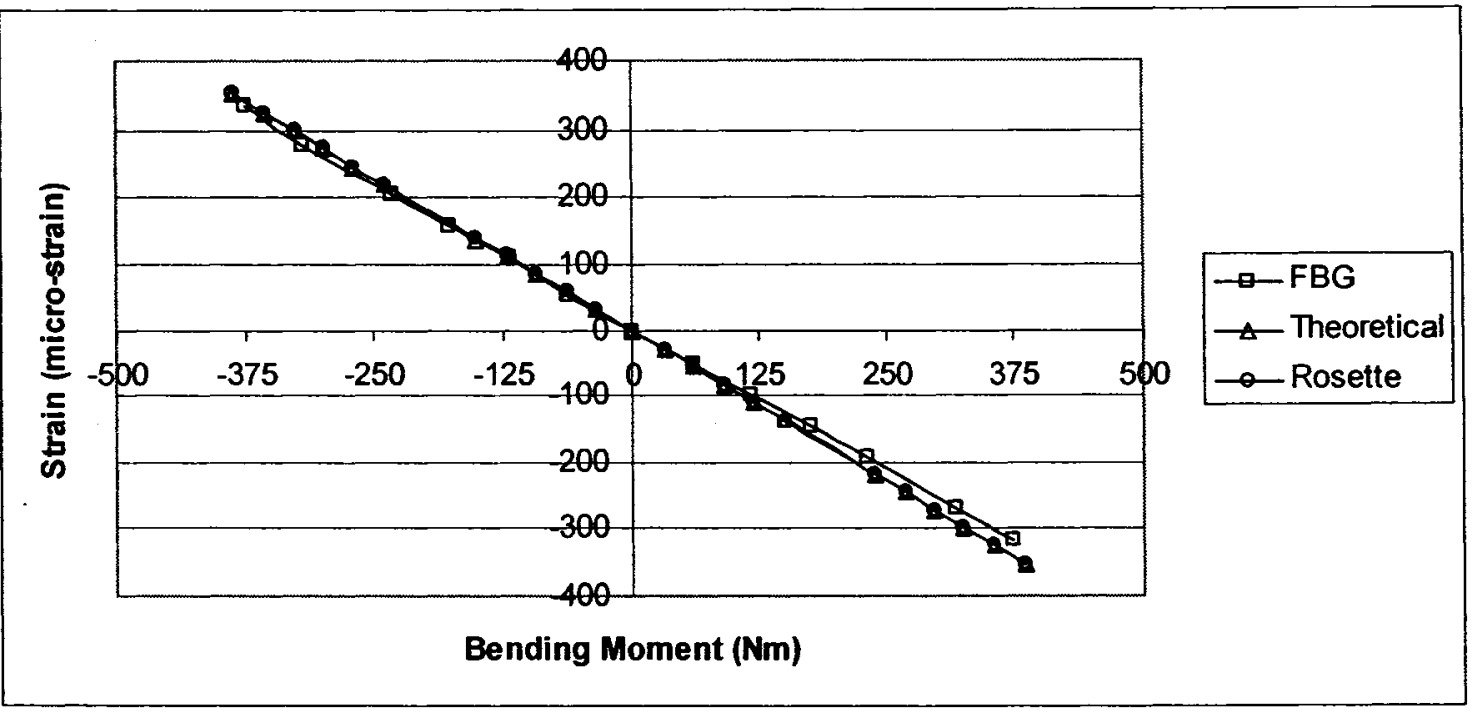

Figure 4.25 Bending results for axial strain on FBG \#6



Figure 4.26 Combined bending and torsion results for axial strain on FBG \#6 


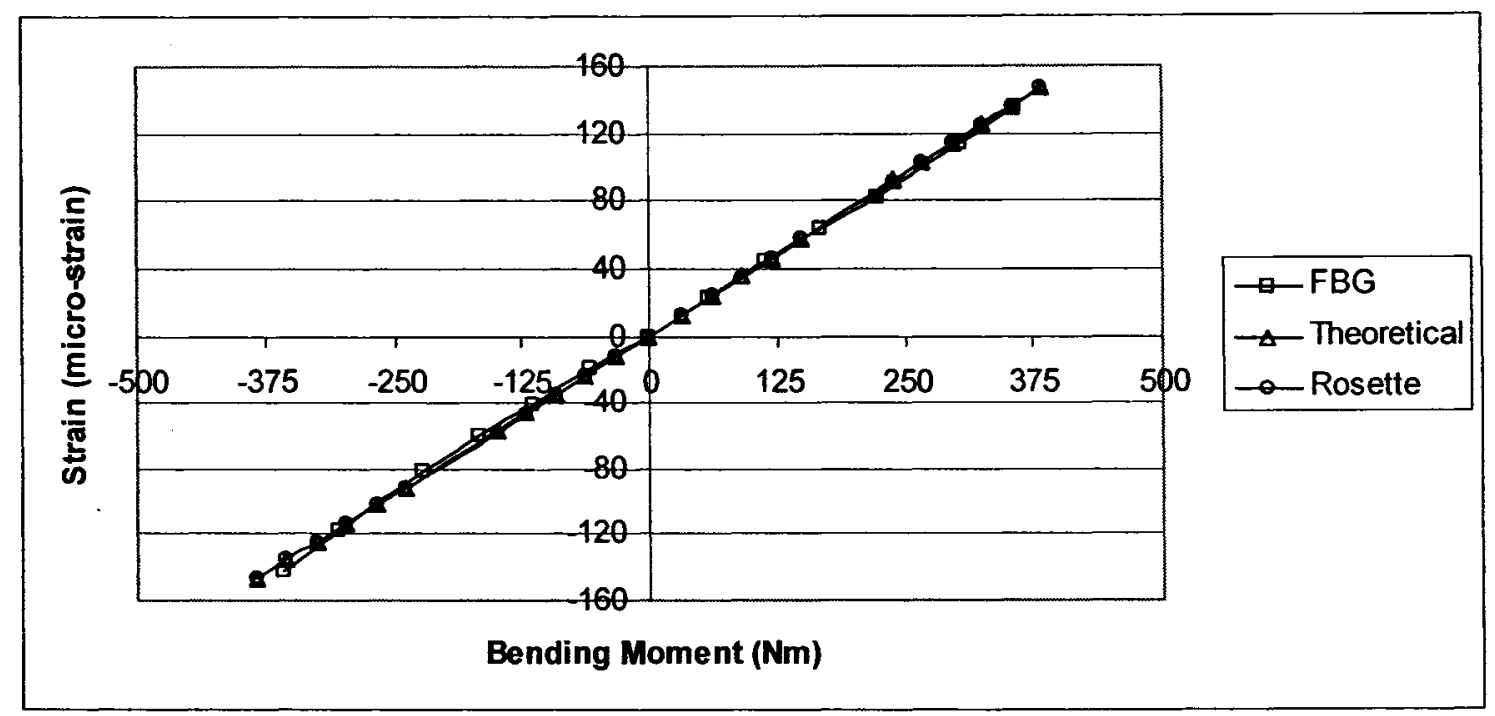

Figure 4.27 Bending results for axial strain on FBG \#7

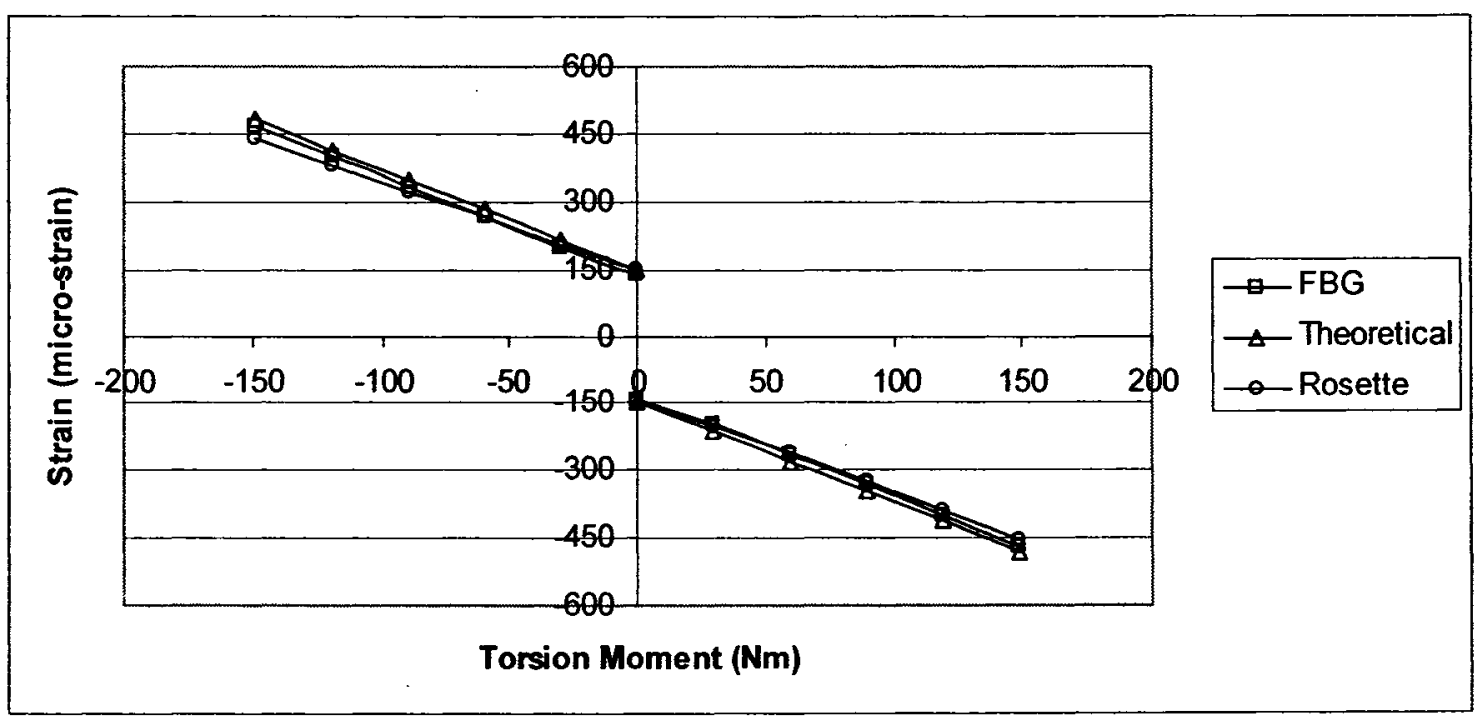

Figure 4.28 Combined bending and torsion results for axial strain on FBG \#7 


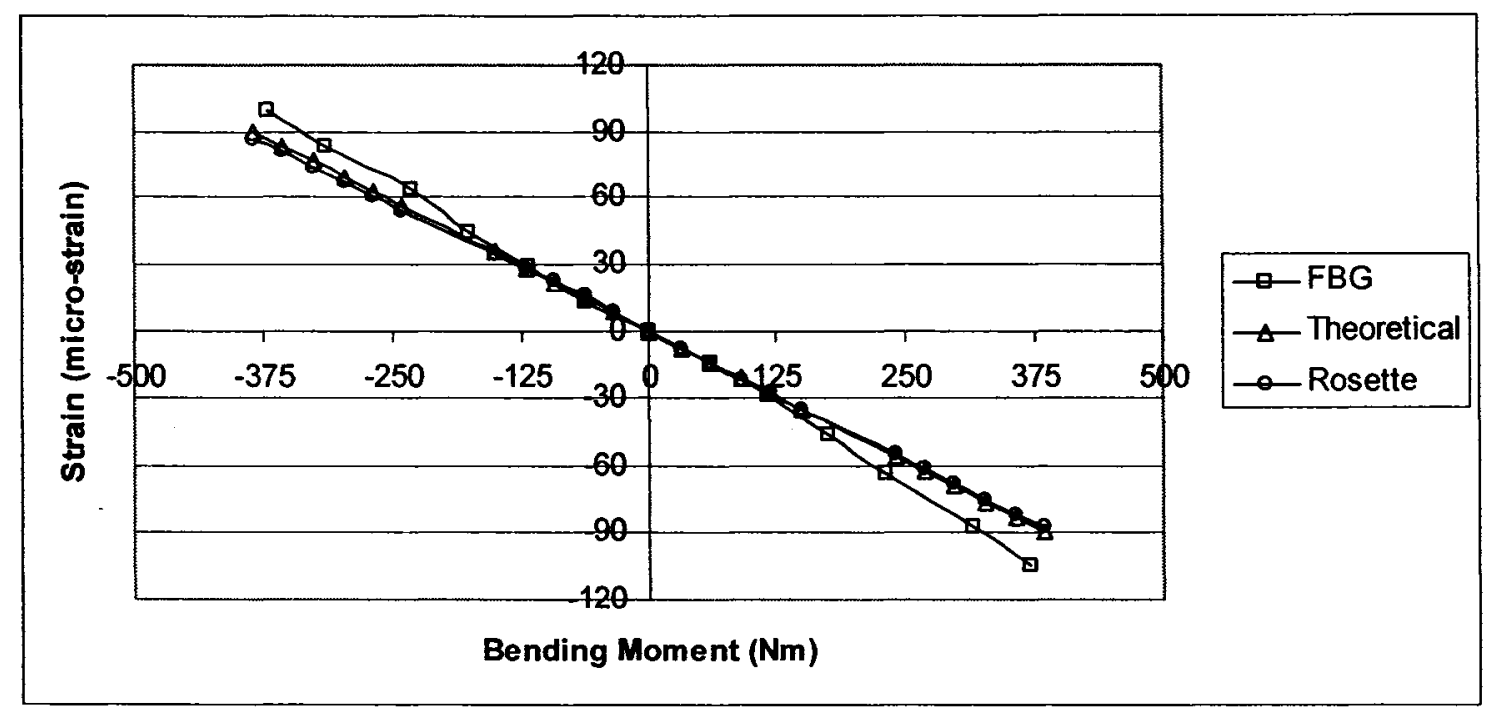

Figure 4.29 Bending results for axial strain on FBG \#8

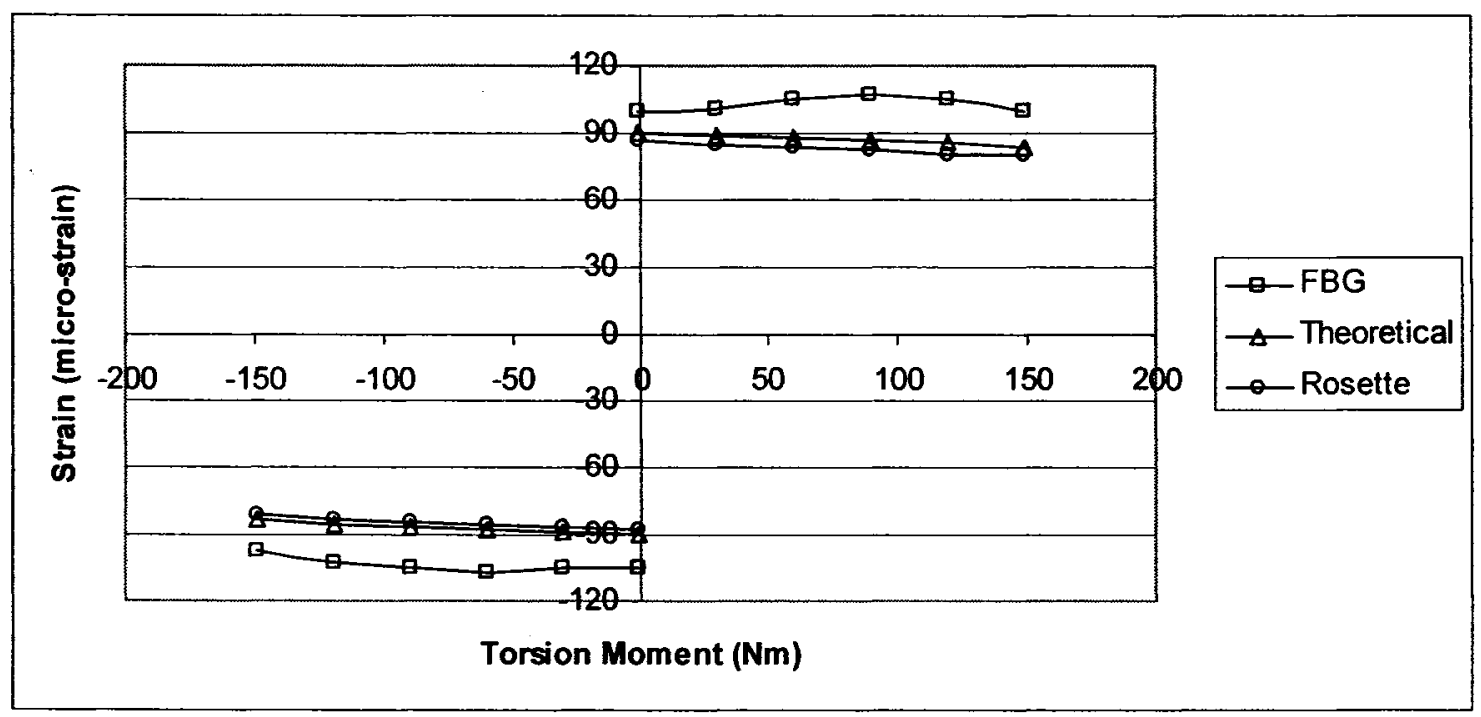

Figure 4.30 Combined bending and torsion results for axial strain on FBG \#8 




Epoxy/Hardener Adhesive

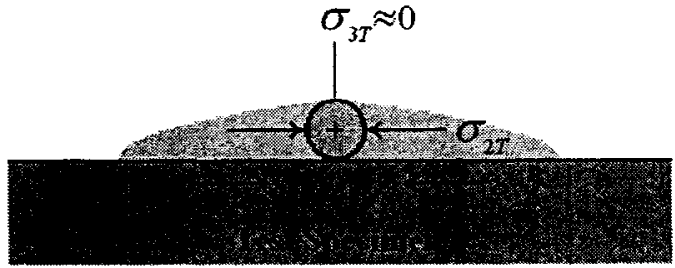

Cyanoacrylate Adhesive

Figure 4.31 Transverse stresses acting on the FBG sensors due to adbesive

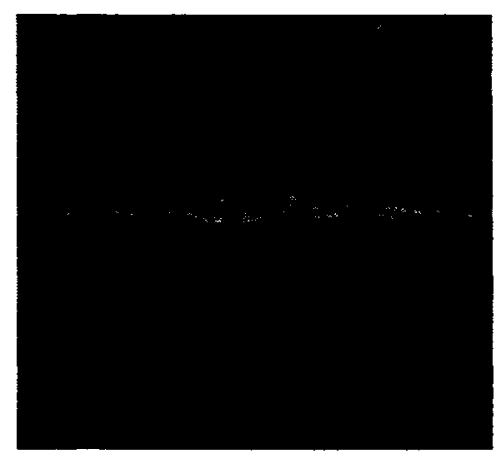

Sensor Embedded at Angle to Fibre Axis

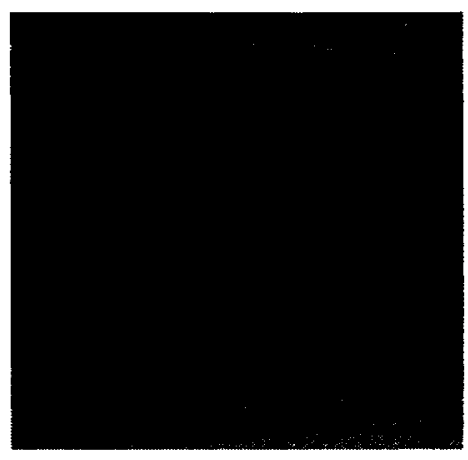

Sensor Embedded Parallel to Fibre Axis

Figure 4.32 FBG sensors embedded in a unidirectional laminate, Fan et al. (2004) 


\section{CHAPTER 5: A PROPOSED FBG SENSOR SYSTEM FOR SHARCS}

\subsection{Introduction}

In this Chapter, an integral dynamic strain sensing system using fibre optics of the SHARCS rotor blade structure is proposed. The structural detail is based on its preliminary design by Mikjaniec (2006). First, the basic requirements of the strain sensing system are established. The system design is then broken down into four subcategories, sensor network, structural integration, data acquisition, and system calibration. The key challenges associated with each sub-category are identified, and solutions proposed where possible. Based on published research and a limited knowledge of current optical component technology, a conceptual system design is presented for the SHARCS rotor. To this end, a general assessment is made with regards to the feasibility and practicality of achieving a fully integral fibre optic sensing system for dynamic strain monitoring of the SHARCS rotor blade, or a similar Mach-scaled rotor blade design in the future. All the calculations performed in this Chapter serve only the purpose of establishing preliminary estimates for the system operating parameters, and are not intended as structural analysis of the SHARCS rotor. 


\subsection{System Design Objectives and Basic Requirements}

The basic system design requirements can be established by examining the desired system performance and the constraints placed on the system by the SHARCS rotor design. The sensory system has three major performance objectives as listed below.

\section{System performance objectives:}

1. dynamic strain monitoring

2. structural vibration monitoring

3. structural health monitoring

In order to achieve integration, the sensory system must conform to the constraints imposed on it by the SHARCS rotor design, which can be summarized into three major areas as listed below.

\section{System constraints:}

1. confined to structural geometry

2. restricted system weight

3. minimal structural intrusiveness

Based on the preliminary SHARCS rotor design presented by Mikjaniec (2006), system operating parameters such as measurement range, speed and resolution will be estimated, followed by a brief look at the system constraints. 


\subsubsection{Measurement Range}

The system should be able to measure strains from zero to maximum rotational speed of the rotor. Estimating the largest strains in the structure during these two extreme load cases will establish a target measurement range for the system. The loads and crosssectional properties used in these estimates are those determined by Mikjaniec (2006).

The maximum strains when stationary are due to static bending moments in the flapping plane, and are related to the axial strain by equation 5.1 .

$$
\varepsilon_{a x}=\frac{M \cdot y}{E I}
$$

- Root $M=-22.5 \mathrm{Nm} \quad y= \pm 4.5 \mathrm{~mm} \quad E I=47.3 \mathrm{Nm}^{2}$

- Blade $M=-20.0 \mathrm{Nm} \quad y= \pm 4.5 \mathrm{~mm} \quad E I=30.8 \mathrm{Nm}^{2}$

The maximum tensile and compressive strains during static bending at zero rotation are estimated to be $\pm 2140 \mu \varepsilon$ and $\pm 2922 \mu \varepsilon$, in the root and blade respectively.

The largest strains during maximum rotation are due to centrifugal forces on the cross-section, and bending moments in the flapping plane.

- Root $F=7500 N \quad M=0.50 N m$

- Blade $F=7400 N \quad M=0.92 N m$

The axial strain due to extension is related to the centrifugal force by equation 5.2 , Mikjaniec (2006), where the subscript $k$ denotes the properties of an individual lamina in the cross-section.

$$
\varepsilon_{a x}=\frac{F}{E A}=\frac{F}{\sum_{k=1}^{N} t_{k} \cdot l_{k}\left(A_{11}+A_{12}^{2} / A_{22}\right)_{k}}
$$


Using the material properties of Table 5.1 and approximating the cross-sectional areas of each laminate $\left(t_{k} \cdot l_{k}\right)$ as flat plates from leading to trailing edge, the products of area and stiffness matrix coefficients were estimated as shown in Table 5.2. The maximum tensile strains due to extension are thereby estimated to be $+650 \mu \varepsilon$ and $+835 \mu \varepsilon$ in the root and blade respectively. The maximum tensile and compressive strains due to bending are found using equation 5.1 to be $\pm 47.6 \mu \varepsilon$ and $\pm 134 \mu \varepsilon$ for the root and blade respectively, and can be superimposed over the extension strains.

The axial strain range in the root is $+2140 \mu \varepsilon$ to $+602.4 \mu \varepsilon$ and $-2140 \mu \varepsilon$ to $+697.6 \mu \varepsilon$ on the top and bottom surfaces respectively, and in the blade $+2922 \mu \varepsilon$ to $+701 \mu \varepsilon$ and $-2922 \mu \varepsilon$ to $+969 \mu \varepsilon$ for the top and bottom respectively. Torsion of the section will add shear strain components to these values, but is not included in this preliminary estimate.

The required system range for axial strains is estimated to be $-3000 \mu \varepsilon$ to $+1000 \mu \varepsilon$, for a total range of $4000 \mu \varepsilon$. If a portion of the blade at the free end was supported prior to rotation and during early spin-up, the strains due to static bending would be significantly lower. This would allow the strain measurement range of the system to be much smaller. Considering an unsupported rotor for now, the range should be increased to, say, $6000 \mu \varepsilon$ to allow for measuring higher strains during vibration of the structure, and account for the uncalculated lead/lag bending and torsion strains on the section. 


\subsubsection{Measurement Speed}

To properly characterize dynamic variations of strain, several sample measurements must be obtained over the course of one vibration cycle, which are assumed to be sinusoidal in form. The sampling rate of the system will limit its ability to recognize structural vibrations of higher frequencies; the system must therefore operate at a minimum speed relative to the structural dynamics of the SHARCS rotor in order to be effective in monitoring structural vibrations. The key natural elastic frequencies of the structure are calculated by Mikjaniec (2006) as listed below in cycles per revolution (per rev) and also in cycles per second $(\mathrm{Hz})$ at the operating speed of the rotor which is $150 \mathrm{rad} / \mathrm{s}$.

\section{Natural Elastic Frequencies:}

- $1^{\text {st }}$ Flapping $\quad 2.55$ per rev $\quad 60.9 \mathrm{~Hz}$

- $2^{\text {nd }}$ Flapping 4.53 per rev $108 \mathrm{~Hz}$

- $1^{\text {st }}$ Lead / Lag 5.33 per rev $127 \mathrm{~Hz}$

- $1^{\text {st }}$ Torsion $\quad 5.82$ per rev $139 \mathrm{~Hz}$

Assuming that a sampling rate of 6 per cycle is the minimum to adequately recognize a sinusoidal form, the system would have to operate at a speed of no less than $834 \mathrm{~Hz}$. A slightly higher sampling rate would improve the systems ability to characterize vibrations of the rotor within this frequency range, and also increase the detectable frequency range of the system. The minimum target operating speed of the system, for a sampling rate of between 7 and 11 per cycle, is $1.0 \mathrm{kHz}$ to $1.5 \mathrm{kHz}$. 


\subsubsection{Measurement Resolution}

In order to monitor dynamic strain variations, the system must be able to distinctly recognize a small change in strain; the smallest measurable change is the system resolution. The system should be able to characterize structural vibrations, which may have very small amplitudes relative to the large axial strain components in the rotor. Based on the experimental results, a target of 5 micro-strain resolution appears to be attainable for the system, which includes the improvement attributed to separation of temperature from the measurements and compensation for transverse sensitivity.

\subsubsection{Structural Geometry}

The sensor network and installation will be confined to the defined geometry of the rotor structure. The laminate and structural geometries of the root and blade sections of the rotor are defined by Mikjaniec (2006) as shown in figures 5.1 and 5.2. The outer geometry is a NACA0012 airfoil $75.3 \mathrm{~mm}$ in chord length; the laminate is built inward from this surface and consists of S-glass/Epoxy and IM6/Epoxy unidirectional preimpregnated laminae, the material properties of which are listed in Table 5.1. Behind the leading edge laminate, a ballast of lead is used to balance the centre of gravity to $1 / 4$ chord position, and the remainder of the internal cavity is filled with a low density foam core material. The root section is assumed to be adhesively bonded onto a titanium mandrel/support-arm, whose geometry is not clearly defined by Mikjaniec (2006), but will be assumed to partially match the inner perimeter of the laminate at the root section near the $1 / 4$-chord position. 


\subsubsection{Weight Restriction}

To achieve a Lock number of 3.7, Mikjaniec (2006) established the target total weight of the SHARCS rotor blade, including all smart-systems, to be $536 \mathrm{~g}$. The structure and ballast designed by Mikjaniec (2006) have a total weight of $400 \mathrm{~g}$, leaving $136 \mathrm{~g}$ for all other systems. As this weight is mostly needed for the smart-systems being installed in the rotor blade, the total weight of the sensor system should be very small. A maximum target weight will be set at $25 \mathrm{~g}$ for sensor system components installed in the rotor itself.

\subsubsection{Structural Intrusiveness}

The embedding of sensors in the laminate should not disrupt the lay-up by creating voids or stress concentrations. The effects on the host structure can be minimized by embedding sensors in unidirectional laminae having a fibre direction in the sensor orientation. The diameter of sensor should be smaller than the thickness of the lamina, so that it will not locally compromise the lamina strength.

\subsubsection{Summary of Design Requirements}

In summary, the target design requirements are as listed below.

- Range $\quad 6000 \mu \varepsilon$

- Speed $\quad 1.0 \mathrm{kHz}$ to $1.5 \mathrm{kHz}$

- Resolution $5 \mu \varepsilon$

- Weight $25 \mathrm{~g}$

- Structure compatible with geometry and non-intrusive 


\subsection{Sensor Network}

The sensor network should be designed such that the sensors are located to achieve each of the performance objectives, while minimizing the total number of sensors needed.

\subsubsection{Dynamic Strain Monitoring Sensors}

As was suggested in Section 4.7, for characterizing accurately the full state of strain at a given point, the ideal placement of sensors into the laminate is to be 'stacked' in unidirectional lamina of three different orientations at the location of interest. Although the loads are higher at the root of the rotor, the strains in the blade section are slightly higher due to the thinner laminate structure. The laminate in the blade section contains three unidirectional lamina orientations, $0^{\circ},+45^{\circ}$, and $-45^{\circ}$, between chord position $0.00 \mathrm{~mm}$ and $21.6 \mathrm{~mm}$ and between $51.4 \mathrm{~mm}$ and $60.7 \mathrm{~mm}$ as shown in figure 5.2. The appropriate place to locate embedded strain sensors is therefore in the blade section beyond rotor position 0226 (Sta. 0226), and between the leading edge and chord position $21.6 \mathrm{~mm}$ or between chord positions $51.4 \mathrm{~mm}$ and $60.7 \mathrm{~mm}$ as shown in figure 5.3 .

Additional sets of sensors can be located within the root section and farther along the blade section in order to provide a more complete distribution of the strains within the whole rotor structure, although these strains will be lower in magnitude. Temperature effects can be separated from the strain measurements by using a dual-overwritten grating among the three sensors at each location. 


\subsubsection{Vibration Monitoring Sensors}

To monitor vibrations in the structure, sensors need to be able to detect the amplitude of the vibrations, as well as the vibration mode. Dyllong and Kreuder (1999) showed that accurate reconstruction of the mode shapes with their amplitudes, up to the $3^{\text {rd }}$ mode can be achieved using a cubic-spline interpolation technique and at least five 'well-placed' non-uniformly spaced sensors along the length of the structure. For accurate reconstruction of only the $1^{\text {st }}$ and $2^{\text {nd }}$ mode shapes, 3 or 4 sensors, respectively, would suffice.

The vibration modes of interest are those corresponding to the natural elastic frequencies given by Mikjaniec (2006); the $1^{\text {st }}$ modes of torsion and lead/lag, and the $1^{\text {st }}$ and $2^{\text {nd }}$ modes of flapping. In order to apply the technique presented by Dyllong and Kreuder (1999), the strain measurements used for interpolation must be dependant only on the vibration mode being examined. The modes must therefore be separated from each other and separated from the steady state strains in the rotor.

Flapping and lead/lag are both bending modes of the rotor section, and produce most noticeably variations in the axial strain. They can be separated from each other and the axial extension of the blade by using four axial strain sensors in a given cross-sectional plane. They would be located on opposing sides of the flapping neutral axis in pairs, each pair at a significantly different distance from the lead/lag neutral axis as shown in figure 5.3. Axial sensors are unaffected by shear strain, thereby making them insensitive to any torsional vibrations. Four sets of axial sensors, in groups of four, would be 
required to accurately reconstruct the $1^{\text {st }}$ lead/lag and the $1^{\text {st }}$ and $2^{\text {nd }}$ flapping mode shapes and amplitudes.

Torsion of the section can be separated from axial extension and bending vibrations by using a pair of sensors at the same location that are oriented at opposing angles to each other, not oriented in the axial direction, as in the case of $+45^{\circ}$ and $-45^{\circ}$ sensors. Any axial or transverse strain applied to this pair due to bending or extension of the section will cause each sensor to measure the same value. Torsion produces shear strain in the section, which causes each sensor to measure differently from the other due to their opposing angles. There is some distributed shear strain caused by bending, however, since the amount due to bending is known, it allows the remainder to be attributed only to torsion. Three pairs of sensors would be required to accurately reconstruct the $1^{\text {st }}$ torsion mode shape and amplitude, and they should be located where the bending of the section can be determined so as to remove the bending shear from the measurements.

According to Dyllong and Kreuder (1999), the sensors must be 'well-placed', meaning that an idea of the expected mode shapes is needed prior to deciding their specific locations. Predicting the mode shapes of the rotor can be achieved using FEM modeling and examining the eigenvector which corresponds to each vibration mode.

\subsubsection{Structural Health Monitoring Sensors}

To monitor structural health, a history of the strain at a given location can be kept in a data log. If the strain begins to change from its typical value, this would indicate the onset of fatigue damage to the structure in the vicinity of the sensor. Sensors for 
structural health monitoring should be placed near locations of stress concentration in the laminate, such as changes in laminate thickness, ply drop-off and pick-up locations, and attachment points of smart-system devices.

\subsubsection{Combining all Sensors into a Single Network}

Considering all the sensor locations described to achieve each objective, the total number of sensors can be minimized for the system by having each sensor in the network contribute to multiple objectives. Figure 5.3 shows a final reduced network with 28 sensors in total; 16 axial strain sensors, four sensors at $+45^{\circ}$ and four sensors at $-45^{\circ}$, and four over-written sensors at $-45^{0}$ to separate temperature effects from the strain measurements.

The 16 axial sensors are able to characterize the $1^{\text {st }}$ and $2^{\text {nd }}$ flapping modes and the $1^{\text {st }}$ lead/lag mode, while the four pairs of $\pm 45^{\circ}$ sensors are able to characterize the $1^{\text {st }}$ torsion mode. There are four locations where the full state of strain can be monitored, independent of temperature effects, one located at each cross-sectional plane, two located on the upper surface, and two located on the lower surface of the rotor. Structural health of the rotor can be monitored by viewing the strain history of any sensor in the network.

The sensors are located at four different cross-sectional planes, each plane having seven sensors; four axial, one at $+45^{\circ}$, one at $-45^{\circ}$, and one over-written at $-45^{\circ}$. The exact location of each plane should be determined using the 'well-placed' locations described by Dyllong and Kreuder (1999) for interpolation of vibration mode shapes, and the locations of largest strains in the structure. The placement of each sensor in the 
laminate and the routing of optical fibre to and from each sensor, as shown in figure 5.3, will be addressed along with other structural integration concerns in the next Section.

\subsection{Structural Integration}

The sensor network should be integrated into the rotor while minimizing the risk of failure to the host structure, the sensors, and the optical fibre network.

\subsubsection{Sensor Failure}

The sensor must be able to withstand the tensile and compressive strains seen in the host material; if the sensor fails before the host material, the sensor would cease to provide useful information. Because FBG sensors are made of silica glass, which is brittle, their failure stress is governed by the probability of a random critical sized flaw in the crystalline structure of the glass. Gougeon et al. (2004) performed dynamic fatigue testing of bare and coated silica fibres to investigate the effects of exposure to humidity. They explain that presence of moisture at the surface of a fibre reduces its failure strength and fatigue life by breaking down the silicon-oxygen bonds at crack tips and accelerating sub-critical crack growth. They found by experiment that the mean failure stress of coated and bare silica fibre to be lower at room temperature and $50 \%$ relative humidity than in a dry environment. The lowest mean failure stress values they reported were 4.74GPa and 8.54GPa for coated and bare fibre respectively. It is assumed that the properties found by Gougeon et al. (2004) are representative of the typical strength of silica glass optical fibre, and thus the strength of FBG sensors. Compared to the tensile strengths and material properties of S-glass/Epoxy and IM6/Epoxy unidirectional lamina, 
as listed in Table 5.1, the sensor is stronger than both, and would not fail prior to the failure of the host material under the same axial strain.

\subsubsection{Host Failure}

If the sensor is small compared to the lamina thickness, its presence will have little effect on the strength of the host structure. The laminae thickness used in the lay-up of the SHARCS structure given by Mikjaniec (2006), as shown in figures 5.1 and 5.2, are approximately $0.500 \mathrm{~mm}$ for the axial oriented S-glass/Epoxy laminae and $0.065 \mathrm{~mm}$ for the $\pm 45^{\circ}$ oriented IM6/Epoxy laminae.

The typical diameter of multi-mode optical fibre is $0.125 \mathrm{~mm}$ with cladding, and between $0.050 \mathrm{~mm}$ and $0.0625 \mathrm{~mm}$ bare, whereas single-mode optical fibre is $0.125 \mathrm{~mm}$ with cladding, and between $0.008 \mathrm{~mm}$ and $0.010 \mathrm{~mm}$ bare. Kojima et al. (2004) have developed a small diameter optical fibre specifically for use as embedded sensors in composite materials, having a core diameter of $0.0065 \mathrm{~mm}$, and with cladding and a polyimide coating, a total diameter of $0.040 \mathrm{~mm}$. Cladding can be chemically stripped from the silica core by immersion in hot sulphuric acid, a process which was shown by Matthewson et al. (1997) to have little effect on the strength of the fibre. Bare singlemode fibre is extremely fragile, and thus difficult to handle without breaking; applying a thin coating to the fibre may provide sufficient reinforcement to allow light handling.

The S-glass laminae are sufficiently thicker than the diameter of multi-mode and single mode fibre, allowing either to be used as embedded sensors. The IM6 laminae are nearly 8 times thinner than the S-glass, and would only allow use of bare single-mode 
sensors with a thin coating without creating a significant structural flaw. If this cannot be achieved due to fibre handling, alternatives are to embed the $\pm 45^{\circ}$ sensors between axial laminae, thus creating resin pockets, or install them on the interior laminate surface.

\subsubsection{Routing of Optical Fibre in the SHARCS Rotor}

The same failure concerns described in Sections 5.3.1 and 5.3.2 apply to the optical fibre used as a conduit to and from the sensor. By taking the most efficient route to each sensor, the amount of optical fibre embedded in the host can be minimized, keeping the weight low, and its structural presence minimal.

The most efficient path for routing optical fibre to the axial sensors is a straight line along the axis of the rotor passing through each sensor. The optical fibre for these sensors can be embedded and routed directly to each sensor location within the laminae because the fibre diameter is small compared to the thickness of the S-glass laminae.

The most efficient path for routing optical fibre to the $\pm 45^{0}$ sensors is a helical-like path around the perimeter of the cross-section while translating down the rotor axis. This would require the optical fibre to turn the leading edge of the cross-section, and the trailing edge, which could be accomplished if a trailing edge wedge, or spar was incorporated into the laminate design. Glaesemann and Castilone (2002) examined the mechanical reliability of bent optical fibre, and list the smallest allowable fibre bend radius as $5 \mathrm{~mm}$, with increasing reliability for larger radii and lower stress levels; a general minimum fibre bend radius of $5 \mathrm{~mm}$ will therefore be assumed. The external radius of the NACA0012 profile at the leading edge of the SHARCS rotor is 
approximately $1.2 \mathrm{~mm}$. For a helical path at either $\pm 45^{\circ}$, the radius of the helical curvature, and thus the bend radius of the fibre, is found to be approximately $2.4 \mathrm{~mm}$ by equation 5.3, which was derived to relate the helical path angle to the radius of the leading edge, Weisstein (2003).

$$
r_{\text {bend }}=r_{L E} \cdot\left(1+\tan ^{2} \theta_{\text {path }}\right)
$$

The radius at the mid-plane of each lamina will also be smaller than the radius of the outer NACA0012 profile, optical fibre at $\pm 45^{\circ}$ can thus not safely turn the leading edge of the rotor. Using equation 5.3 , the leading edge radius would have to be approximately $2.5 \mathrm{~mm}$, or the fibre angle would have to be less than $\pm 29^{\circ}$ for the fibre to safely turn the leading edge. Unless the laminate was redesigned with $\pm 29^{0}$ lamina orientations, each $\pm 45^{0}$ sensor must therefore be installed on its own optical fibre in the network, and the optical fibre must be routed to the sensor location taking a path outside the lamina and then enter the lamina prior to the sensor.

Routing the optical fibre between laminae would disturb the laminate and create locations of structural weakness and must be avoided. The SHARCS laminate is built on foam core, as shown in figures 5.1 and 5.2 , leaving access to the $\pm 45^{\circ}$ laminae edges directly from the foam core between chord positions $30.6 \mathrm{~mm}$ and $44.6 \mathrm{~mm}$. This allows the optical fibre to be safely routed below, or depressed into, the foam surface as shown in figure 5.3, without significantly affecting the laminate. The optical fibre would travel down the axis of the rotor, and then turn to the appropriate orientation and enter the lamina at chord position $30.6 \mathrm{~mm}$. The turn radius of the fibre in the foam, and thus the 
fibre bend radius, should be as generous as possible, as Wang et al. (2005) showed that bend radii less than $12 \mathrm{~mm}$ create additional macro-bending loss in single-mode fibre.

As was suggested in Section 5.3.2, the thickness of the IM6 laminae at $\pm 45^{\circ}$ is so thin that it would only allow bare single-mode optical fibre with a thin coating to be embedded into it. Because the optical fibre will be routed to the sensor location through the foam, the cladding does not need to be removed from the entire optical fibre, only the end section of the fibre containing the sensor which will be embedded in the lamina. Even though the sensor will still be quite fragile, this makes handling and installation of the $\pm 45^{0}$ fibre more manageable.

Although it is desired to monitor the complete state of strain at the locations with high stresses, embedding sensors into the laminate at those locations may weaken it and cause failure, especially when considering the extremely thin IM6 lamina. The bottom surface of the blade section, immediately beyond the root section of the rotor is under the highest tensile loads at maximum rotation, and the highest compressive loads when stationary. It is therefore decided, on the side of caution, to install the $\pm 45^{\circ}$ sensors on the upper surface for the two most inboard cross-sections, and to balance the number of optical fibres routed in the laminate on each surface, the $\pm 45^{\circ}$ sensors are located on the bottom surface for the two outboard cross-sections. Also, because the $\pm 45^{\circ}$ sensors are each on their own optical fibre, it is more convenient for data acquisition to use them as the temperature discriminating dual over-written gratings.

There are 12 optical fibres in the sensor network of each rotor blade, and can all originate from a common fibre optic ribbon cable bonded to the titanium mandrel/support 
arm. The fibres can then separate from each other, fan out around the titanium mandrel, and then enter into the laminae or the foam core at their respective entry points.

\subsection{Data Acquisition}

Data acquisition deals with the part of the system concerning the transmission and receipt of optical signals, and how the system actually interrogates the sensor network.

\subsubsection{Transmitting and Receiving Optical Signals}

Having integrated a sensor network into the structure, an optical signal must be sent to and retrieved from each sensor. This requires a continuous optical wave guide, or optical fibre path from the source, to the sensor, and then back to the detector. This creates a challenge for using fibre optic sensors in a rotor blade because the sensors are located in a rotating frame of reference. There are two possible solutions to this problem, use a fibre optic rotary joint (FORJ) to allow rotating optical fibres to maintain an optical path with non-rotating fibres, or to have the source and detector units rotating with the rotor blade.

Commercially available FORJ devices, such as those manufactured by Schleifring Ltd., are capable of rotational speeds up to $2000 \mathrm{rpm}$ for a single fibre FORJ, but only up to speeds of $100 \mathrm{rpm}$ for a multi-fibre FORJ. As there are several optical fibres used in the network of each blade, and four blades, a multi-channel device would be needed; it is not possible to use many single FORJ devices because the optical fibres would tangle around each other. The rotor speed is $1433 \mathrm{rpm}$, thus current FORJ technology can not solve the problem. The source and detector units must therefore rotate with the rotor blades at $1433 \mathrm{rpm}$, requiring some form of structural integration with the rotor hub. 
This will be the greatest challenge of the entire system design, as the system resolution, range, and speed are all highly dependant on the source and detector units.

\subsubsection{Optical Circuit Components}

Conventional optical sources and detectors, such as tuneable lasers and spectrum analyzers are not capable of meeting the speed requirement, and are also too bulky to be mounted onto or rotated with the rotor structure. Ling et al. (2006) demonstrated a simple, fairly compact passive interrogation scheme for measuring dynamic strains of a FBG sensor using a super luminescent diode (SLD) as a source, and passing the signal through an optical tuneable filter (OTF). The OTF causes optical signal loss proportional to the wavelength of light, therefore as the centre wavelength of the FBG shifts, the optical power measured by the detector will shift proportionally. Dyllong and Kreuder (1999) demonstrated a similar system using a broadband light source and a wavelength dependant coupler (WDC) which performs a similar function to the OTF. Zhang et al. (1998) also demonstrated a similar system, but using a long period grating (LPG), in place of the OTF and WDC. A LPG causes loss to the optical transmission signal over a fairly wide band as shown in figure 5.4. If the reflected signal of a FBG sensor was transmitted through a stable LPG, the power loss to the Bragg peak would be proportional to its centre wavelength. Zhang et al. (1998) splits the reflected signal from the FBG sensor into two, applies the LPG to only one of the signals, and measures each signal using its own photo-detector. The ratio of power measured by the detector behind the LPG to the power measured by the unaffected detector indicates the centre wavelength position of the Bragg peak. This accounts for wavelength dependant power 
variation in the source, source modulation, and other signal losses throughout the system which may change from time to time, such as connector and micro-bend losses.

Using a similar interrogation scheme to either of these three, would meet the speed requirement for the SHARCS system. Zhang et al. (1998) also showed that their passive interrogation system using a LPG has a large range of approximately $10,000 \mu \varepsilon$ and a very high resolution of approximately $\pm 0.5 \mu \varepsilon$, which more than adequately meets the range and resolution requirements for the SHARCS system. Because a LPG is an intrinsic all-fibre device, similar to a FBG, using them would have the added advantage of reducing the component size and weight of the system. Figure 5.5 shows a basic optical circuit similar to that of Zhang et al. (1998) which uses an LPG interrogation scheme. Most of the circuit components shown in figure 5.5 can be obtained in very compact forms, leading to a very compact optical circuit, which is ideal for the SHARCS system.

The source device could be a light emitting diode (LED) or a SLD. Both are extremely compact, widely available, and fairly inexpensive. A SLD is capable of emitting significantly higher optical power than a LED, typically as high as $10 \mathrm{~mW}$ to $20 \mathrm{~mW}$, compared to a LED at $500 \mu W$ to $1 \mathrm{~mW}$. Their output powers are proportional to the electrical drive current applied to them, which can be modulated, or turned on and off, at very high speeds, up to $100 \mathrm{MHz}$ according to Shidlovski (2004).

Optical splitters and couplers are available in compact form, for example, Fujikura Ltd. has mini-couplers which are $28 \mathrm{~mm}$ long and $3 \mathrm{~mm}$ diameter, and Lightcomm Technology Co. has mini-couplers as small as $18 \mathrm{~mm}$ long and $2 \mathrm{~mm}$ diameter. These 
devices are fused-couplers, and can be built to divide the input signal in any proportion, evenly or unevenly into to any number of outputs; the optical power is divided among the outputs, and some optical power is lost due to excess losses in the coupler.

There are several options available for compact detectors, among which photo-diodes (PD) appear to be the most appropriate choice for the SHARCS system. Godfrey (1998) compares the characteristics of many different photo-detector devices, and thereby provides a basis for selecting the most appropriate device for a given system design. Godfrey (1998) describes the typical characteristics of a PD as small, lightweight, low cost, rugged, very sensitive with the ability to measure from the $p W$ to the $m W$ range of optical power, high linearity, able to detect a broad range of wavelengths, stable, and can be easily packaged and customized at a fairly low cost to suit any design.

By using these compact components to replace those of figure 5.5, the same passive interrogation circuit as Zhang et al. (1998) would be achieved, but in a miniaturized form. The basic optical circuit would require a pair of PD detectors, one LED or SLD source, one LPG filter, and two mini-splitters for interrogation of a single FBG sensor. Applying this circuit design to the SHARCS system for each sensor would however result in 28 LED sources, 28 LPG filters, 56 mini-splitters and 56 PD detectors for each of the four rotor blades. By sharing sources and detectors among sensors, and combining splitters, the total number of components in the system can be significantly reduced. The result is a more complex system architecture, but one that is overall more efficient in use of system resources, size, and weight. The next section describes a conceptual design for 
the data acquisition system of the SHARCS sensory system, which uses the passive LPG interrogation technique and as few components as possible.

\subsubsection{SHARCS Sensory System Concept}

Some trade-offs can be made to the system performance in order to reduce the number of required components. For example, by intermittently interrogating one out of every four sensors, only 14 PD detectors would be needed, at the penalty of reducing the system speed below one quarter its maximum. This can be achieved using four sources, where each is filtered to generate only a spectral window corresponding to one out of four sensors. Because LED and SLD sources can be modulated at speeds up to $100 \mathrm{MHz}$, this allows each of the four sensors to be interrogated in rapid succession using the same set of detectors. Even at one quarter its maximum speed, the system is still more than capable of fulfilling the SHARCS speed requirement.

Figure 5.6 shows a conceptual system using the LPG passive interrogation technique to monitor all 28 sensors in the SHARCS network, while significantly reducing the number of required components. The system shares four common sources, each filtered to generate a different spectral window, which are then modulated so that only one source is active at any given time. The 28 sensors are divided into four groups, each group corresponds to one of the four cross-sectional planes shown in figure 5.3, and all seven sensors of that group have centre wavelengths which correspond to the same spectral window of one of the four sources. Thus, when any given source is active, only the seven sensors from the corresponding cross-sectional plane will generate an optical response. All seven sensors of a given group are simultaneously and continuously monitored by 
seven pairs of detectors, where each detector pair corresponds to one sensor in the group. The 14 detectors are common to all four groups, leaving control of which sensors are active at any time to the source modulation. One of the two detectors in each pair is located behind a series of LPG filters that correspond with the four spectral windows of the sources and sensors. The four spectral windows are spaced so that they correspond only to a linear portion of the LPG transmission signal, as shown in figure 5.4. Because the transmission peak of a LPG has a rise on one side and a fall on the other, as shown in figure 5.4, a single LPG can be used for two or more spectral windows. Therefore, one or two LPG filters may be sufficient for all four spectral windows. Should multiple LPG filters be used, their signals should not interfere with the linear regions of the other filters. A special consideration must be given to the dual-overwritten sensors which are used to separate temperature from the strain measurements, as both sensors are in the same fibre and need to be interrogated simultaneously. This is only achievable using two sets of detectors in parallel, and filtering out the optical response of the unwanted sensor prior to each detector.

This system uses the passive interrogation technique on the detector side, while the only actively controlled devices in the system are the sources which are modulated. This allows some flexibility in system operation; one source can remain constantly active to continuously monitor a single cross-sectional plane, two sources can be modulated at any desired frequency, or they can all be modulated as desired. Optical signals from a given source can only be split so many times before the power at the detector is insufficient after all the system power losses. This system concept allows modifications as needed, to 
add additional sources, detectors or sensors, and compensate for the actual performance of each system component to power losses.

\subsection{System Calibration}

If the system was completely closed and integrated into the SHARCS rotor, it would be very difficult to monitor its performance and ensure it is accurately measuring the strains. A source may fail, a detector may cease to work properly, or a sensor itself may fail or debond. It is therefore essential for the continuing operation and accuracy of the system that it be able to be calibrated from time to time with relative ease.

The simplest method to achieve this is to make the entire system modular, and easily separable. Figure 5.6 shows that the conceptual system is separated into three modules, the source module, the detector module, and the sensor module. Only the sensor module is integrated into the SHARCS rotor, and consists of the sensors and the optical fibre routed to each sensor location. All the optical fibres originate from a fibre optic ribbon cable on the titanium support arm, which can be terminated with a multiple fibre connector, such as the MT, MTP, MPO-type connectors. This type of connector is very compact, and allows for all 12 optical fibres to be simultaneously connected and disconnected easily and quickly to other devices with a matching connector. The detector and source module can be fitted with the same type of connectors, thereby allowing each module to be easily separated and calibrated individually using other optical sources, detectors, and spectrum analyzers, or replaced if needed. 
There are three other areas of concern regarding system calibration and performance, namely, transverse sensitivity of the sensors, wavelength dependence of fused couplers/splitters, and influence of temperature on the transmission spectrum of a LPG.

\subsubsection{Sensor Transverse Sensitivity}

As described in Section 4.6.2, the elastic interaction of the sensor with the host lamina can affect its transverse sensitivity. The material coefficients $k_{E l}, K_{13 l}, K_{23 l}$ for each lamina used in the SHARCS structure are listed in Table 5.4. These values are used to approximate the axial and transverse sensitivity factors $F_{a}, F_{t}$ and the corresponding optical gauge factor and transverse sensitivity $F_{o p t}, k_{o p t}$ of an embedded FBG sensor, following the example of Section 4.6.3, and are listed in Table 5.4. The transverse sensitivity for an embedded FBG sensor is estimated to be $-1.6 \%$ and $-2.0 \%$ for the Sglass and IM6 lamina respectively. These values can be verified by laminate tensile test coupons made of the respective lamina materials and tested in an MTS machine while simultaneously measuring the optical response of embedded FBG sensors.

\subsubsection{Wavelength Dependence of Fused Couplers/Splitters}

The power splitting ratio of a fused coupler is typically wavelength dependant. As the wavelength of the FBG shifts, the change in power splitting ratio of the last coupler before the detector pair would cause a 'false' strain indication for that sensor. The wavelength variation of the coupler is typically provided by the manufacturer and can be easily verified by testing it with a wavelength tuneable source. This effect can then be accounted for in the measurements of each sensor. 


\subsubsection{Temperature Response of a LPG}

Bhatia (1999) showed in figure 5.4, that the transmission spectrum of a LPG shifts in wavelength with temperature changes. This would cause the interrogation system to indicate a 'false' sensor strain. A simple solution is to use an internal temperature sensor circuit in the detector unit, as shown in figure 5.6, using the same passive interrogation technique and a LPG, to determine the temperature in the detector. Output from this circuit can then be used to compensate for the temperature shifts of the other LPG devices in the system. An external connector is included so this circuit can be calibrated.

\subsection{Concluding Remarks}

Based on the SHARCS preliminary structural design by Mikjaniec (2006), a basic set of system design requirements was established for an integral dynamic strain sensing system using fibre optics. A preliminary sensor network was established which would meet the multiple sensing objectives; it consists of 28 embedded FBG sensors using 12 singlemode optical fibres. Based on the high-speed dynamic FBG interrogation techniques developed by several researchers and available miniature optical components, a conceptual data acquisition system was presented which should be able to meet all the system requirements and performance objectives. A fully integral fibre optic sensing system for dynamic strain monitoring of the SHARCS rotor blade thus appears to be attainable using fairly inexpensive devices. It would, however, require detailed design and calibration. It remains to determine the ideal locations of the sensors, which requires a reasonably accurate prediction of the expected strain components and deflection shapes of the rotor blade; this could be achieved using finite element modeling. 
Table 5.1 Lamina material properties used for SHARCS rotor - Mikjaniec (2006)

\begin{tabular}{lll}
\hline Property & S-glass / Epoxy & IM6 Carbon Fibre / Epoxy \\
\hline$v_{f}$ & 0.5 & 0.66 \\
$\rho\left(g / \mathrm{cm}^{3}\right)$ & 2.00 & 1.60 \\
$E_{1}(G P a)$ & 43 & 203 \\
$E_{2}(G P a)$ & 8.9 & 11.2 \\
$G_{12}(G P a)$ & 4.5 & 8.4 \\
$v_{12}$ & 0.27 & 0.32 \\
$F_{1 T}(M P a)$ & 1280 & 3500 \\
$F_{2 T}(M P a)$ & 49 & 56 \\
$F_{1 C}(M P a)$ & 690 & 1540 \\
$F_{2 C}(M P a)$ & 158 & 150 \\
$F_{6}(M P a)$ & 69 & 98 \\
\hline
\end{tabular}

Table 5.2 Lamina stiffness matrix coefficients

\begin{tabular}{|c|c|c|c|c|}
\hline \multirow{2}{*}{$\begin{array}{l}\text { Coefficient (GPa) } \\
\text { Orientation }\end{array}$} & \multirow{2}{*}{$\begin{array}{l}\text { S-glass / Epoxy } \\
0\end{array}$} & \multicolumn{3}{|c|}{ IM6 Carbon Fibre / Epoxy } \\
\hline & & $\mathbf{0}$ & $+45^{\circ}$ & $-45^{\circ}$ \\
\hline$Q_{11}$ & 45.55 & 206.65 & 95.98 & 95.98 \\
\hline$Q_{12}$ & 12.30 & 66.13 & 79.18 & 79.18 \\
\hline$Q_{22}$ & 9.43 & 11.40 & 95.98 & 95.98 \\
\hline$Q_{16}$ & 0 & 0 & 48.81 & -48.81 \\
\hline$Q_{26}$ & 0 & 0 & 48.81 & -48.81 \\
\hline$Q_{66}$ & 4.5 & 8.4 & 50.30 & 50.30 \\
\hline
\end{tabular}


Table 5.3 Approximate extension stiffness matrices of SHARCS rotor

\begin{tabular}{lll}
\hline Stiffness Coefficient \& Area Product & Root Laminate & Blade Laminate \\
\hline$\sum_{k=1}^{N} t_{k} \cdot l_{k}\left(A_{11}\right)_{k}\left(G P a \cdot m m^{2}=N \cdot 10^{3}\right)$ & 7821 & 5798 \\
$\sum_{k=1}^{N} t_{k} \cdot l_{k}\left(A_{12}\right)_{k} \quad\left(G P a \cdot m^{2}=N \cdot 10^{3}\right)$ & 4003 & 3457 \\
$\sum_{k=1}^{N} t_{k} \cdot l_{k}\left(A_{22}\right)_{k} \quad\left(G P a \cdot m^{2}=N \cdot 10^{3}\right)$ & 4321 & 3902 \\
$\sum_{k=1}^{N} t_{k} \cdot l_{k}\left(A_{16}\right)_{k} \quad\left(G P a \cdot m^{2}=N \cdot 10^{3}\right)$ & $0($ balanced $)$ & 0 (balanced) \\
$\sum_{k=1}^{N} t_{k} \cdot l_{k}\left(A_{26}\right)_{k} \quad\left(G P a \cdot m^{2}=N \cdot 10^{3}\right)$ & 0 (balanced) & 0 (balanced) \\
$\sum_{k=1}^{N} t_{k} \cdot l_{k}\left(A_{66}\right)_{k} \quad\left(G P a \cdot m^{2}=N \cdot 10^{3}\right)$ & 2222 & 2022 \\
\hline
\end{tabular}

Table 5.4 Sensitivity coefficients for embedded FBG sensor in lamina

\begin{tabular}{lll}
\hline Coefficient & S-glass / Epoxy & IM6 Carbon Fibre / Epoxy \\
\hline$k_{E l}$ & 0.113 & 0.138 \\
$K_{13}$ & $-0 . .356$ & -0.418 \\
$K_{23}$ & -0.320 & -0.307 \\
$F_{a}$ & 0.7936 & 0.7949 \\
$F_{t}$ & -0.0125 & -0.0156 \\
$F_{o p t}$ & 0.7811 & 0.7793 \\
$k_{\text {opt }}$ & -0.01575 & -0.019625 \\
\hline
\end{tabular}


SHARCS - Root Lay-up : Sta. 0126-0226

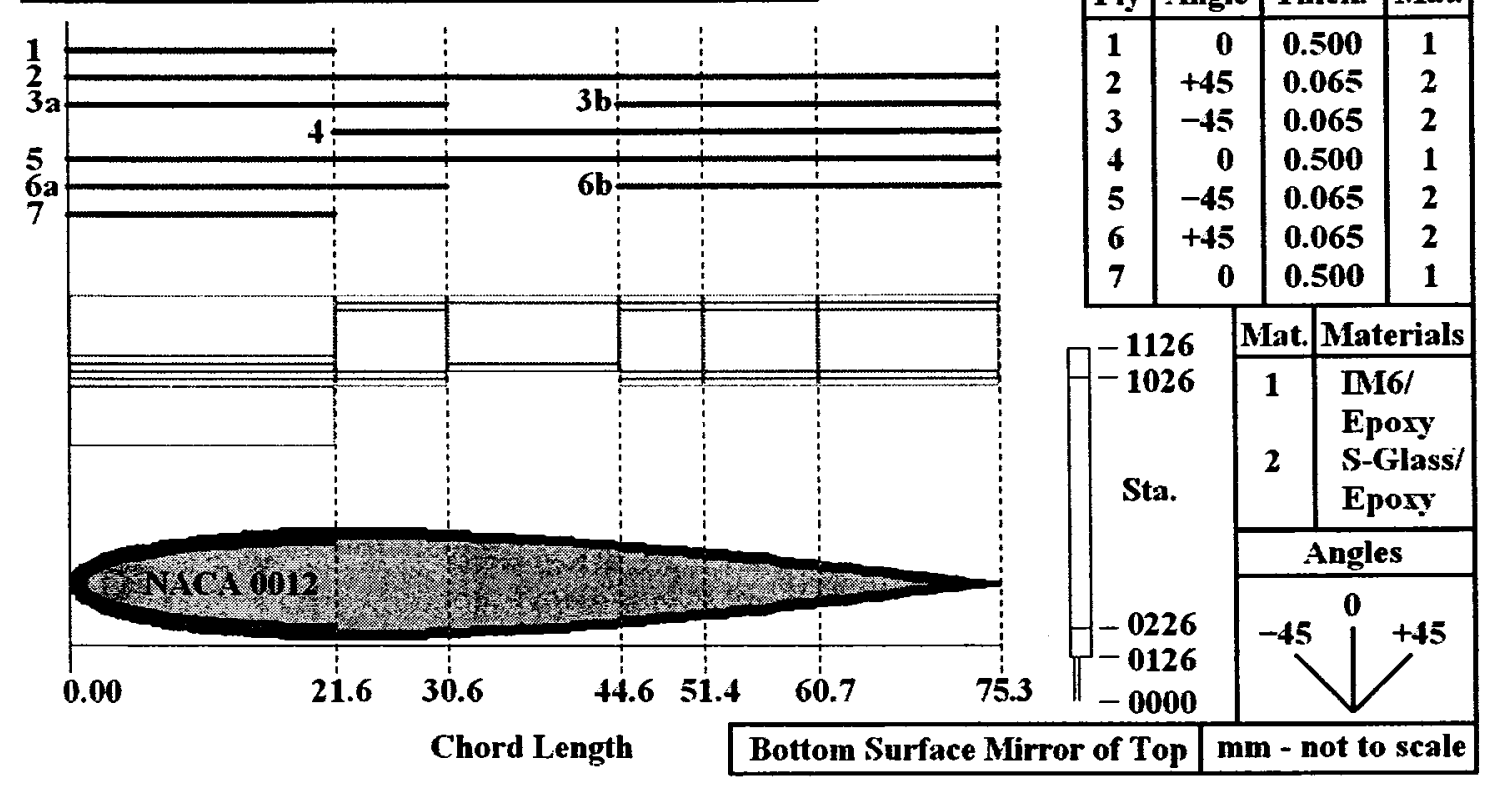

Figure 5.1 Laminate lay-up for the root of SHARCS rotor - Mikjaniec (2006)

SHARCS - Blade Lay-up : Sta. 0226-1026

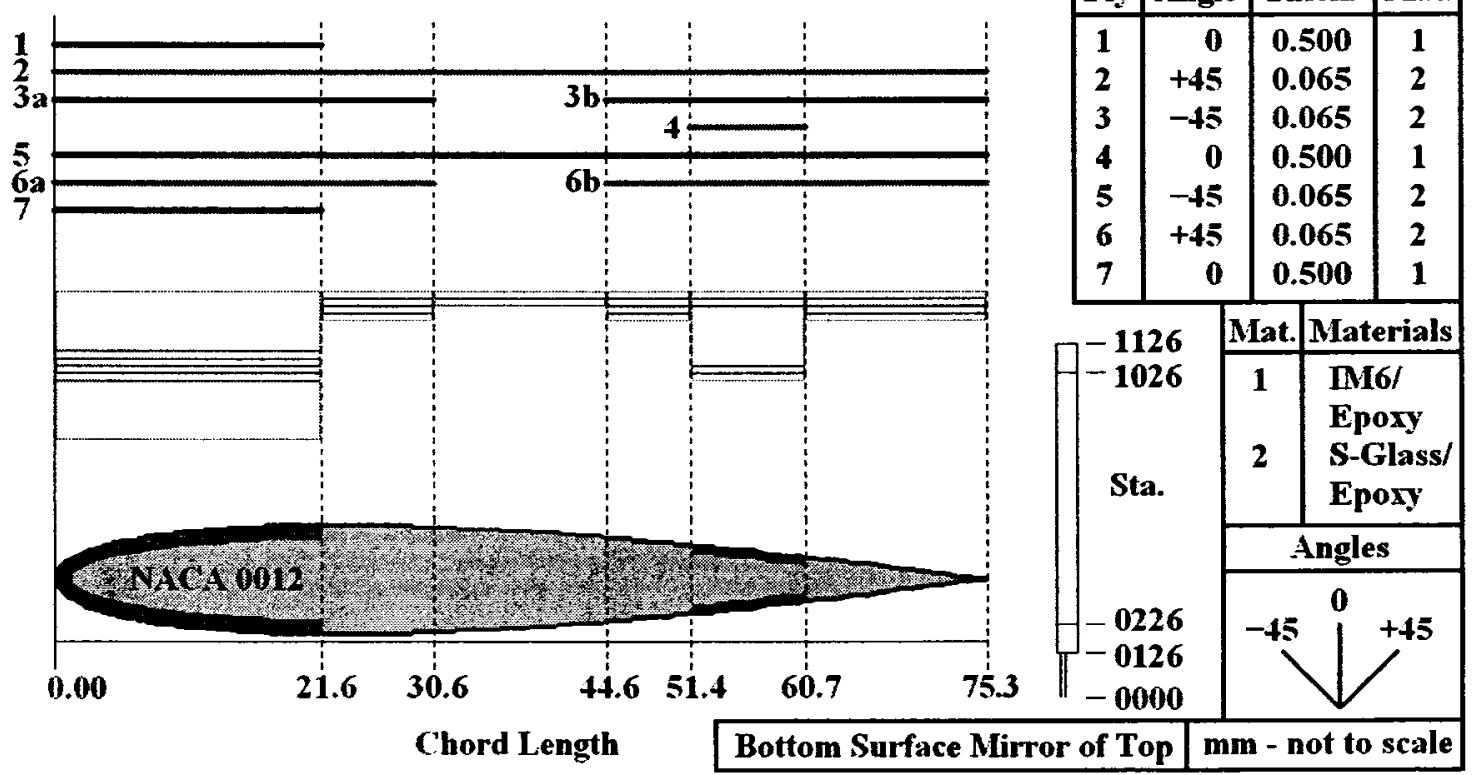

Figure 5.2 Laminate lay-up for the blade of SHARCS rotor - Mikjaniec (2006) 


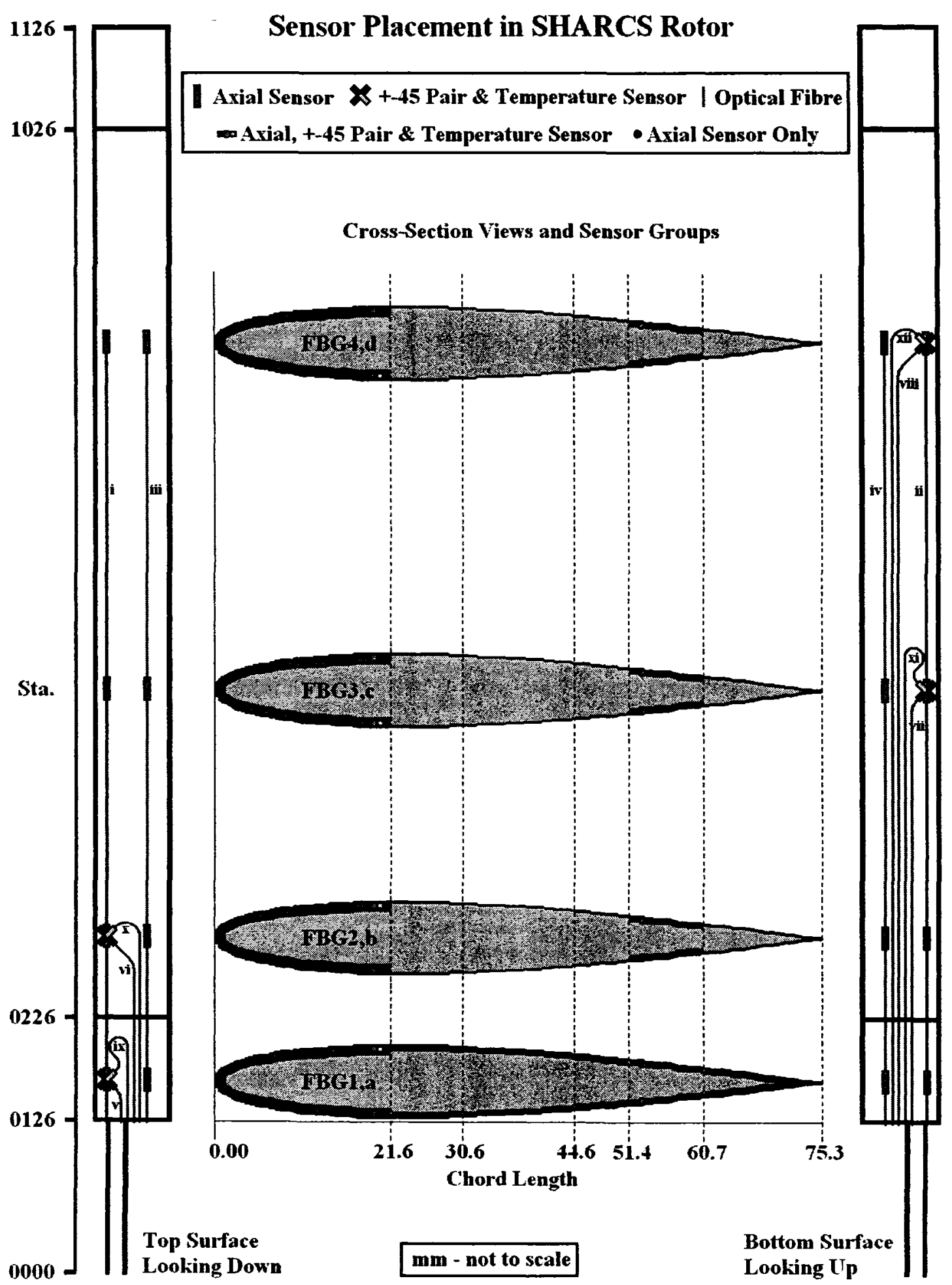

Figure 5.3 Sensor placement and optical fibre routing for SHARCS rotor 


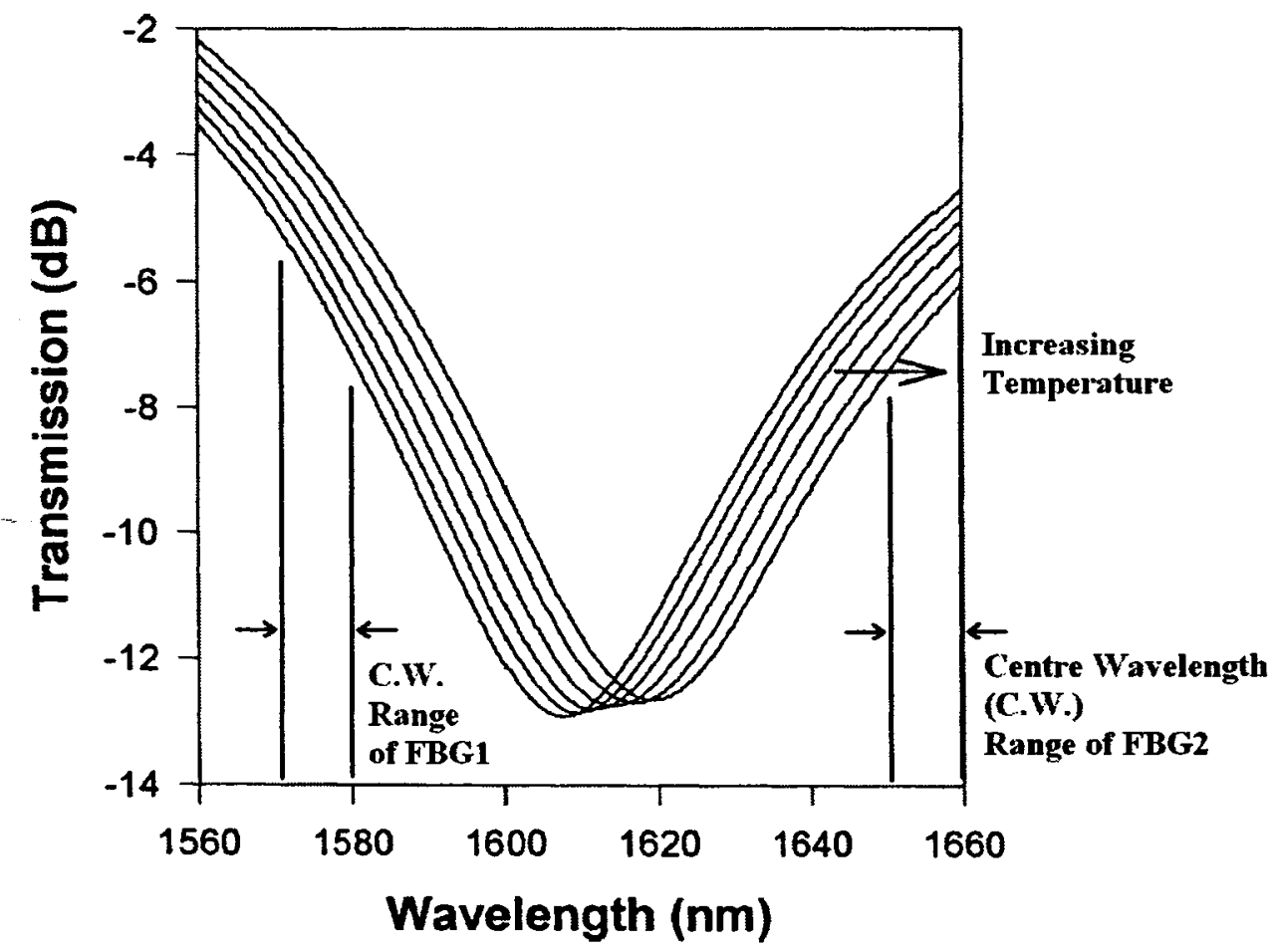

Figure 5.4 Long period grating transmission signal - Bhatia (1999)
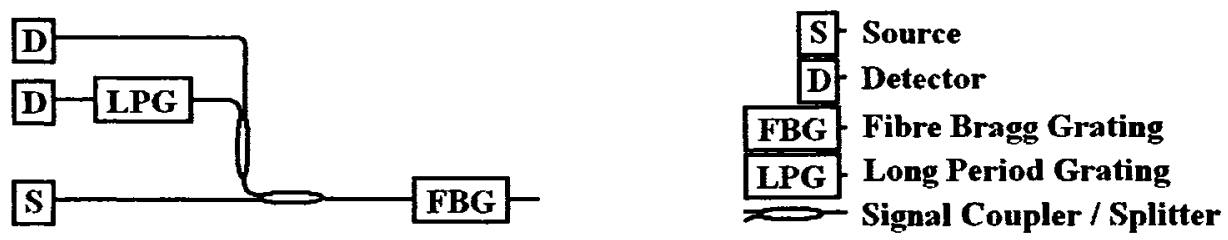

Figure 5.5 Basic LPG interrogation circuit for a FBG sensor 


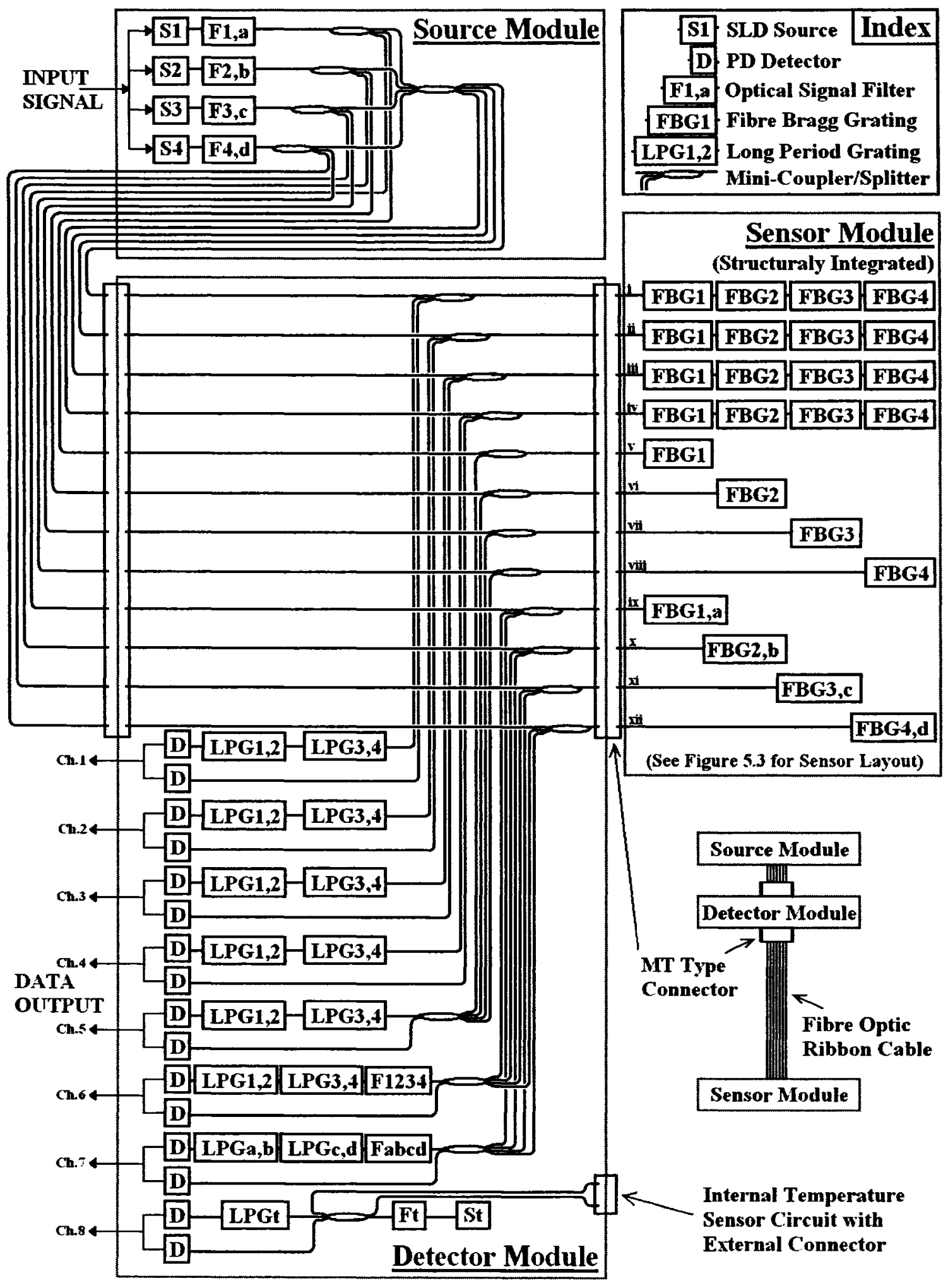

Figure 5.6 Conceptual data acquisition system for SHARCS 


\section{CHAPTER 6: CONCLUSIONS}

From the literature review of fibre optic strain sensory technology, fibre Bragg gratings were selected as the most suitable sensor devices to be used as embedded point strain sensors in a Smart Hybrid Active Rotor Control System (SHARCS) rotor blade application. This is because of their low intrusiveness and high multiplexing abilities. The strain sensing abilities of these optical fibre Bragg gratings (FBG) and their theoretical behavior were verified through a series of experimental tests which were developed. In these tests, multiple FBG sensors were adhesively installed on the surfaces of cantilever test specimens, to which combined bending and torsional loads were applied. The cantilever beams were loaded in bending and torsion and strains were also obtained using several electrical resistance strain gauge rosettes which provided comparative strain measurement data for the FBG sensor results.

The experimental results showed excellent agreement with the predicted theoretical values, exhibiting high linearity and numerical accuracy over small strain ranges. Primary sources of measurement error were attributed to temperature effects and transverse sensitivity. Both of these sources of error can be significantly reduced by using a simple temperature compensation technique and estimating the transverse 
sensitivities of the FBG sensors, which are based on the elastic interaction of the sensor with the surrounding host materials. This was demonstrated in the present work.

A conceptual dynamic strain sensing system and sensor network was presented for the SHARCS rotor blade application. It was based on a preliminary structural design of the rotor blade by Mikjaniec (2006). It follows the techniques employed by several researchers, the use of available compact optical components to meet a basic set of system design requirements. In a more detailed design of the system as a next step, it is suggested that a finite element analysis of the SHARCS rotor blade be performed to establish more accurately the limiting strains in order to determine the appropriate FBG system requirements.

\subsection{Recommendations for Future Work}

In continuing research on the topic of fibre optic strain sensors embedded in composite materials and to fully realize an integral dynamic fibre optic strain sensing system within a mach scaled helicopter rotor blade, such as that proposed in Chapter 5 of this work, there are several areas of research which should be addressed in more detail.

A boundary element or finite element study could be used to investigate the effect of the resin pocket geometry on the transverse sensitivity of embedded FBG sensors. This parametric study would serve as support for the theoretical optical response of FBG sensors embedded in laminate structures.

A finite element model analysis of the rotor blade would establish more accurately the system design requirements, and assist with sensor placement within the structure. The finite element model would also serve as a link between the measured strains and the 
loads acting on the rotor blade, accounting for coupling effects due to the anisotropy of laminate lay-up and coupling caused by the structural dynamics and large deflections of the rotor blade.

Integration of the sensor module within the rotor blade structure requires addressing several practical issues, such as ingress/egress of the optical fibre into the laminate, the routing of optical fibre to the sensing locations, the details of manufacturing and assembly with regards to the laminate lay-up, temperature exposure and fading of the FBG sensors during the cure cycle of the laminate, and application of any specialized coatings which may required. These issues would be addressed through a series of practical investigations, which could also include laminate coupon testing with embedded FBG sensors to assess their measurement repeatability and any fatigue of the sensors.

Finally, assembly and calibration of the source and detector modules would require as a first step proof of concept testing of the basic interrogation circuit, shown in figure 5.5, using the compact components described in Chapter 5. After testing the basic interrogation circuit, more complex circuit concepts can be built and tested, leading to the final verification and testing of the source and detector module concepts illustrated in figure 5.6. 


\section{References}

Agilent Technologies (1996), "Optical Spectrum Analysis". Application Note 1550-4, Santa Clara, California, USA

Alahbabi, M.N., Lawrence, N.P., Cho, Y.T., and Newson, T.P. (2004). "High spatial resolution microwave detection system for Brillouin-based distributed temperature and strain sensors". Measurement Science and Technology. 15, pp. 1539-1543

ANSYS, version 10.0 Help File, 2006

Atkins, R.M. (1996). Photosensitivity in Optical Fibers, Lucent Technologies-Bell Laboratories, Murray Hill, New Jersey, USA.

Baek, S., Jeong, Y., and Lee, B. (2002). "Characteristics of short-period blazed fiber Bragg gratings for use as macro-bending sensors". Applied Optics. 41, pp. 631-636

Bertholds, A., and Dandliker, R. (1988). "Determination of the Individual Strain-Optic Coefficients in Single-Mode Optical Fibers". Journal of Lightwave Technology. 6, pp. $17-20$

Bhatia, V. (1999). "Applications of long-period gratings to single and multi-parameter sensing”. Optics Express. 4, pp. 457-466

Carson, M. (2006). "Characterizing polarization-dependant wavelength in AWGs and PLCs". Application Note 155, EXFO Electro-Optical Engineering Inc. 
Caucheteur, C., Lhomme, F., Maakaroun, F., Chah, K., Blondel, M., and Megret, P. (2004). "Simultaneous strain and temperature sensor using superimposed tilted Bragg gratings". Proceedings Symposium IEEE/LEOS Benelux Chapter. pp. 219-222

Chen, X., Zhou, K., Zhang, L., and Bennion, I. (2005). "Optical Chemosensor Based on Etched Titled Bragg Grating Structures in Multimode Fiber". IEEE Photonics Technology Letters. 17, pp. 864-866

Dong, X., Meng, H., Liu, Z., Kai, G., and Dong, X. (2001). "Bend measurement with chirp of fiber Bragg grating". Smart Materials and Structures. 10, pp. 1111-1113

Dyllong, E., Kreuder, A. (1999). "Optimal reconstruction of mode shapes using nonuniform strain sensor spacing". Proceedings of the 1999 IEEE/ASME International Conference on Advanced Intelligent Mechatronics

Eaton, N.C., Drew, R.R., and Geiger, H (1995). "Finite element stress and strain analysis in composites with embedded optical fiber sensors". Smart Materials and Structures. 4, pp.114-117

Erdogan, T. (1997). "Fiber Grating Spectra". Journal of Lightwave Technology. 15, pp. 1277-1294

Fan, Y., and Kahrizi, M. (2004). "Applicability of an Optimized Fiber Optic Smart Structure". Proceedings of the 2004 IEEE International Conference on MEMS, NANO and Smart Systems (ICMENS'04)

Friebel, E.J., Askins, C.G., Bosse, A.B., Kersey, A.D., Patrick, H.J., Pogue, W.R., Putnam, M.A., Simon, W.R., Tasker, F.A., Vincent, W.S., and Vohra, S.T.(1999). 
"Optical fiber sensors for spacecraft applications". Smart Materials and Structures. 8, pp.813-838

Ghatak, A., and Thyagarajan, K.(1998). Introduction to Fiber Optics, Cambridge University Press, Cambridge, United Kingdom.

Giles, C. R. (1997). "Lightwave Applications of Fiber Bragg Gratings". Journal of Lightwave Technology. 15, pp. 1391-1404

Glaesemann, S., and Castilone, R.J. (2002). "The mechanical reliability of Corning optical fiber in bending". White paper: WP3690, Corning Inc., New York, USA.

Godfrey, L. (1998). "Choosing the detector for your unique light sensing application". PerkinElmer Inc.

Gornall, W., and Amarel, T. (2003). Applications and techniques for fiber Bragg grating sensor measurements, EXFO, Burleigh Products Group, Victor, New York, USA.

Green, A.K., Zaidman, M., Shafir, E., Tur, M., and Gali, S. (2000). "Infrastructure development for incorporating fibre-optic sensors in composite materials". Smart Materials and Structures. 9, pp.316-321

Grobnic, D., Mihailov, S.J., Smelser, C.W., and Ding, H. (2004). "Sapphire fibre Bragg grating sensor made using femtosecond laser radiation for ultrahigh temperature applications". IEEE Photonics Technology Letters, 16, pp. 2505-2507

Grossman, B.G., and Huang, L.T. (1998). "Fiber optic sensor array for multi-dimensional strain measurement". Smart Materials and Structures. 7, pp.159-165 
Gougeon, N., Poulain, M., and Abdi, R. El (2004). "Evolution of strength silica optical fibers under various moisture conditions". Optical Materials. 27, pp. 75-79

Hadjiprocopiou, M., Reed, G.T., Hollaway, L., and Thorne, A.M. (1996). "Optimization of fibre coating properties for fiber optic smart structures". Smart Materials and Structures. 5, pp.441-448

Hecht, J. (2006). Understanding Fiber Optics, Prentice Hall, New Jersey, USA

Hetenyi, M. (1950). Handbook of Experimental Stress Analysis, John Wiley \& Sons, Inc., New York, USA

Hill, K.O., Fujii, Y., Johnson, D.C., and Kawasaki, B.S. (1978). "Photosensitivity in optical fiber waveguides: Application to reflection filter fabrication". Applied Physics Letters. 32, pp. 647-49

Hill, K.O. and Meltz, G. (1997). "Fiber Bragg Grating Technology Fundamentals and Overview". Journal of Lightwave Technology. 15, pp. 1263-1276

Kalamkarov, A.L., Georgiades, A.V., MacDonald, D.O., and Fitzgerald, S.B. (2000). "Pultruded fibre reinforced polymer reinforcements with embedded fibre optic sensors". Canadian Journal of Civil Engineering. 27, pp. 972-984

Kang, H.K., Park, J.W., Ryu, C.Y., Hong, C.S., and Kim, C.G. (2000). "Development of fibre optic ingress/egress methods for smart composite structures". Smart Materials and Structures. 9, pp. 149-156 
Kang, S.C., Kim, S.Y., Lee, S.B., Kwon, S.W., Choi, S.S., and Lee, B.H. (1998).

"Temperature-Independent Strain Sensor System Using a Tilted Fiber Bragg Grating Demodulator". IEEE Photonics Technology Letters. 10, pp. 1461-1463

Kersey, A.D., Davis, M.A., Patrick, H.J., LeBlanc, M., Koo, K.P., Askins, C.G., Putnam, M.A., and Friebele, J. (1997). "Fiber Grating Sensors". Journal of Lightwave Technology. 15, pp. 1442-1463

Kim, N.-S., and Cho, N.-S. (2004). "Estimating Deflection of a Simple Beam Model Using Fiber Optic Bragg-grating Sensors”. Experimental Mechanics. 44, pp. 433-439

Kojima, S., Komatsuzaki, S., Kurosawa, Y., and Hongo, A. (2004). "Embedding type strain sensors using small-diameter fiber Bragg grating to composite laminate structures". Hitachi Cable Review No. 23 (August 2004), Advanced Technology Laboratories, Hitachi Cable, Ltd., Japan

Laffont, G., and Ferdinand, P.(2001). "Tilted short-period fibre-Bragg-grating-induced coupling to cladding modes for accurate refractometry". Measurement Science and Technology. 12, pp. 765-770

Lau, K.T., Yuan, L., and Zhou, L.M. (2001). "Thermal effects on an embedded grating sensor in and FRP structure". Smart Materials and Structures. 10, pp. 705-717

Lin, Y.B., Chang, K.C., Chern, J.C., and Wang, L.A. (2005). "Packaging Methods of Fiber-Bragg Grating Sensors in Civil Structure Applications". IEEE Sensors Journal. 5, pp.419-424 
Ling, H.-Y., Lau, K.-T., Cheng, L., and Jin, W. (2006). "Viability of using an embedded FBG sensor in a composite structure for dynamic strain measurement". Measurement. 39, pp. 328-334

Magne, S., Rougeault, S., Vilela, M., and Ferdinand, P. (1997). "State-of-strain evaluation with fiber Bragg grating rosettes: application to discrimination between strain and temperature effects in fiber sensors". Applied Optics. 36, pp. 9437-9447

Mall, S., Dosedel, S.B., and Holl, M.W. (1996). "The performance of graphite-epoxy composite with embedded optical fibers under compression". Smart Materials and Structures. 5, pp. 209-215

Matthewson, M.J., Kurkjian, C.R., and Hamblin, J.R. (1997). "Acid stripping of fused silica optical fibers without strength degradation". Journal of Lightwave Technology. 15, pp. $490-497$

Mat-Web (2007). Online material properties data-base www.matweb.com

Meltz, G., Morey, W.W., and Glenn, W.H.(1989). "Formation of Bragg gratings in optical fibers by a transverse holographic method". Optics Letters. 14, pp. 823-825

Mikjaniec, T. (2006). "The Preliminary Design of the SHARCS Rotor Blade". M.A.Sc. Thesis, Department of Mechanical and Aerospace Engineering, Carleton University, Ottawa, Ontario

Mottram, J.T.(2004). "Shear Modulus of Standard Pultruded Fiber Reinforced Plastic Material". Journal of Composites for Construction. 8, pp. 141-147 
NEETS (2006). "Introduction to Fibre Optics". NEETS Module 24, Integrated

Publishing, Port Richey, Florida, USA

Oliver, W.C., Pharr, G.M. (1992). "An Improved Technique for Determining Hardness and Elastic Modulus Using Load and Displacement Sensing Indentation Experiments". Journal of Materials Research. 7, pp. 1564-1583

Pak, Y.E. (1992). "Longitudinal shear transfer in fiber optic sensors". Smart Materials and Structures. 1, pp. 57-62

Peters, K., Studer, M., Botsis, J., Iocco, A., Limberger, H., and Salathe, R.(2001). "Embedded Optical Fiber Bragg Grating Sensor in a Nonuniform Strain Field: Measurements and Simulations". Experimental Mechanics. 41, pp. 19-28

Prabhugoud, M., Peters, K. (2006). "Finite element model for embedded fiber Bragg grating sensor". Smart Materials an Structures. 15, pp. 550-562

Riley, W.F., Sturges, L.D., and Morris, D.H. (1995). Statics and Mechanics of Materials, John Wiley \& Sons Inc., New York, USA

Rourk, R.J. (1975). Formulas for Stress and Strain, McGraw Hill, Inc., New York, USA

Shidlovski, V. (2004). "Superluminescent diodes. Short overview of device operation principles and performance parameters." SuperlumDiodes Ltd., Moscow, Russia

Shivakumar, K., and Emmanwori, L. (2004). "Mechanics of failure of composite laminates with an embedded fiber optic sensor". Journal of Composite Materials. 38, pp. $669-680$ 
Shivakumar, K., and Bhargava, A. (2005). "Failure mechanics of a composite laminate embedded with a fiber optic sensor". Journal of Composite Materials. 39, pp. 777-798

Shroeder, K., Ecke, W., Apitz, J., Lembke, E., and Lenschow, G. (2006). “A fibre Bragg grating sensor system monitors operational load in a wind turbine rotor blade". Measurement Science Technology. 17, pp. 1167-1172

Simpson, A.G., Zhou, K., Zhang, L., Everall, L., and Bennion, I. (2004). "Optical sensor interrogation with a blazed fiber Bragg grating and a charge-coupled device linear array". Applied Optics. 43, pp. 33-40

Sirkis, J.S.(1993). "Optical and mechanical isotropies in embedded fiber optic sensors". Smart Materials and Structures. 2, pp. 255-259

Skontorp, A. (2002). "Composites with embedded optical fibers at structural details with inherent stress concentrations". Journal of Composite Materials. 36, pp. 2501-2515

Smith, K.H., Ipson, B.L., Loweder, T.L., Hawkins, A.R., Selfridge, R.H., and Schultz, S.M. (2006). "Surface-relief fiber Bragg gratings for sensing applications". Applied Optics. 45, pp. 1669-1675

Stewart, A., Carman, G., and Richards, L. (2003). "Nondestructive Evaluation Technique Utilizing Embedded Thermal Fiber Optic Sensors". Journal of Composite Materials. 37, pp. 2197-2206

Surgeon, M., and Weavers, M. (2001). "The influence of embedded optical fibres on the fatiqgue damage progress in quasi-isotropic CFRP laminates". Journal of Composite Materials. 35, pp.931-940 
Tian, X.G., and Tao, X.M.(2001). "Torsion Measurement Using Fiber Bragg Grating Sensors". Experimental Mechanics. 41, pp.248-253

Tuttle, M.E. (2004). Structural Analysis of Polymeric Composite Materials, Marcel Dekker, Inc., New York, USA

Udd, E., (1991), Fiber Optic Sensors, An Introduction for Engineers and Scientists, John Wiley \& Sons, Inc., New York, USA.

Wang, Q., Farrell, G., and Freir, T. (2005). "Theoretical and experimental investigations of macro-bend losses for standard single mode fibers". Optics Express. 13, pp. 4476-4484

Weisstein, E.W. (2003). "Helix". Mathworld - a Wolfram Web Resource.

Xiao, H., Deng, J., Pickrell, G., May, R.G., and Wang, A. (2003). "Single-Crystal Sapphire Fiber-Based Strain Sensor for High-Temperature Applications". Journal of Lightwave Technology. 21, pp. $2276-2283$

Zhang, L., Fallon, R., Everall, L.A., Williams, J.A.R., and Bennion, I. (1998). "LargeDynamic-Range and High-Resolution from a Strain Sensing System using Long-Period Grating Interrogating FBG Strain Sensor'. ECOC'98. 20-24 September 1998, Madrid, Spain 